BOSTON UNIVERSITY

GRADUATE SCHOOL OF ARTS AND SCIENCES

Dissertation

\title{
MEASUREMENT OF THE ANOMALOUS MAGNETIC MOMENT OF THE NEGATIVE MUON TO 0.7 PARTS PER MILLION
}

by

JONATHAN M. PALEY

B.A., Drew University, 1997

Submitted in partial fulfillment of the

requirements for the degree of

Doctor of Philosophy

2004 

Approved by

First Reader

B. L. Roberts, Ph.D.

Professor of Physics

Second Reader

Robert M. Carey, Ph.D.

Associate Professor of Physics 



\section{DEDICATION}

This thesis is dedicated to my parents, Adam and Suzanne Paley, my first and best teachers. Thank you for your love, support, and encouragement. 



\section{ACKNOWLEDGMENTS}

There are so many people I wish to thank who have in one way or another made this thesis possible. I wish to give special thanks to my advisor, Professor B. Lee Roberts, for his many thoughtful comments on the content of this thesis and for his support, educational and otherwise, during my graduate school career. I am also extremely grateful to Robert Carey, the second reader of this thesis. His insight, eagerness to discuss physics and moral support have helped to guide me along the path to finishing my Ph.D. Thanks to James Miller, for his sincere interest and support of my work. Thanks also to my non-(g-2) thesis committee members, Professors Karl Ludwig and Kenneth Lane. I thank Kevin Lynch for the many theory discussions we have had lately, and for his technical support this last year. I also wish to thank the entire BU Physics Department faculty and staff for making my years as a graduate student so enjoyable. I have also learned much from my fellow BU peers, and I thank them for their support: Alexei Trofimov, Matthew Hare, Anatoly Gafarov, Xiaobo Huang, Qinzeng Peng, and Michael Hance.

I wish to thank the entire Muon $(g$-2) Collaboration - their eagerness to do good physics, and the many thought-provoking conversations we have had as a group over the years has taught me much. In particular, I thank Ivan Logashenko, Ernst Sichtermann, Ofer Rind, Mario Deile, Gerco Onderwater, Fred Gray, Chris Polly, Dave Hertzog, William Morse, Yannis Semertzidis, Francis Farley, Cenap Özben, Long Duong, Ben Bousquet, and Petr Shagin. I owe a huge debt of gratitude to my colleagues who made the 2001 data run and all the work that went into this thesis so much fun!

The love and support I have received from my family and friends has played a critical role in the completion of this thesis. I thank my parents Adam and Suzanne, and my siblings Alexandra, Andrew and Shari for their unwaivering and enthusiastic support over the years. I also wish to thank my dear friends and fellow physicists Robert Badzey and Alfredo Aranda for their support and comradery. To my love, Laura Barnes, I thank you for your patience, support and understanding. I wish I could name all my other friends and loved ones who have been there for me during graduate school, but there are simply too many to list... I truly appreciate and thank you all.

Experiment E821 at BNL was supported in part by the U.S. Department of Energy, the U.S. National Science Foundation, the German Bundesminister für Bildung und Forschung, the Russian Ministry of Science, and the U.S.-Japan Agreement in High Energy Physics. Funding for the work on this thesis was provided by the U.S. National Science Foundation's grant to the BU Intermediate Energy Group. 



\title{
MEASUREMENT OF THE ANOMALOUS MAGNETIC MOMENT OF THE NEGATIVE MUON TO 0.7 PARTS PER MILLION
}

\author{
(Order No. )
}

\section{JONATHAN M. PALEY}

Boston University Graduate School of Arts and Sciences, 2004

Major Professor: B. L. Roberts, Professor of Physics

The present generation of measurements of the anomalous magnetic moment of the muon $\left(a_{\mu}\right)$ have reached sub part per million ( $\left.\mathrm{ppm}\right)$ precision, a level at which they are sensitive to electromagnetic and hadronic interactions, and for the first time, to the electroweak interactions. Comparing the experimental results with Standard Model evaluations provides stringent constraints on physics beyond our current model. The determination of $a_{\mu}$ at Experiment 821 at Brookhaven National Laboratory requires simultaneous measurements of the muon spin precession frequency and the magnetic field of the muon storage ring. This analysis, one of several to measure the spin precession frequency, uses a ratio of phase-shifted decay electron time spectra to unshifted time spectra. Combined with an independent measurement of the magnetic field, the anomalous magnetic moment of the negative muon has now been determined to a precision of 0.7 parts per million (ppm):

$a_{\mu^{-}}=11659214(8)(3) \times 10^{-10}$. This value is in good agreement with measurements of the anomalous magnetic moment of the positive muon: $a_{\mu^{+}}=11659204(7)(5) \times 10^{-10}(0.7$ $\mathrm{ppm})$. We discuss the principle of and the analysis techniques used in this experiment, and compare the final results with the theoretical prediction for $a_{\mu}$. 



\section{Contents}

1 Introduction $\quad 1$

1.1 Background on the Anomalous Magnetic Moment . . . . . . . . . . . . 1

1.1.1 Some Definitions . . . . . . . . . . . . . . . . . 1

1.1.2 The Electron $g-2 \ldots \ldots \ldots \ldots \ldots \ldots$

1.2 QED Contributions to $a_{\mu} \ldots \ldots \ldots \ldots \ldots$

1.3 Weak Contributions to $a_{\mu} \ldots \ldots \ldots \ldots \ldots$

1.4 Hadronic Contributions to $a_{\mu} \ldots \ldots \ldots \ldots$

1.4.1 First order vacuum polarization contribution . . . . . . . . 6

1.4 .2 Higher order hadronic contributions . . . . . . . . . . . . . . 11

1.5 Sensitivity of $a_{\mu}$ to Physics Beyond the Standard Model . . . . . . . . . . 13

2 Experiment E821 at BNL $\quad 15$

2.1 Previous Measurements of $a_{\mu} \ldots \ldots \ldots \ldots \ldots$

2.2 Principle of the Experiment . . . . . . . . . . . . . . . 16

2.3 Production and Injection of Polarized Muons . . . . . . . . . . . . . 19

2.3.1 AGS Beamline . . . . . . . . . . . . . . . . . . 19

2.3.2 The Superconducting Inflector . . . . . . . . . . . . . . . . . . . 22

2.4 Storage of Muons . . . . . . . . . . . . . . . . . . . 24

2.4.1 The Muon g-2 Storage Ring . . . . . . . . . . . . . . . . 24

2.4.2 Muon Injection and Beam Dynamics . . . . . . . . . . . . . . . . 25

2.4.3 Monitoring the Muon Beam . . . . . . . . . . . . . . . . . . 40

2.4.4 Muon Injection and Beam Dynamics Simulation . . . . . . . . . . 43

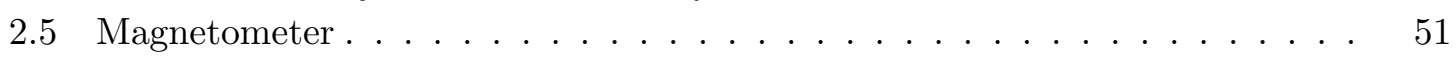

2.5.1 Nuclear Magnetic Resonance . . . . . . . . . . . . . . . 53

2.5.2 NMR Probes . . . . . . . . . . . . . . . . . 55

2.5.3 The Trolley . . . . . . . . . . . . . . . . . . . . 56

2.5.4 Data Acquisition System _. . . . . . . . . . . . 58

2.6 Muon Decay Detector System . . . . . . . . . . . . . . . . . . 59

2.6 .1 Electron Detectors . . . . . . . . . . . . . . . . . 59

2.6.2 Data Acquisition System _. . . . . . . . . . . . . . 62

2.6.3 Data Production . . . . . . . . . . . . . . . . . 64

2.7 Previous Results of E $821 \ldots \ldots \ldots \ldots \ldots$ 
32001 Magnetic Field Measurement $\quad 69$

3.1 Standard Probe Calibration . . . . . . . . . . . . . . . . 69

3.2 Trolley Probe Calibration and Measurements . . . . . . . . . . . . . 70

3.3 Fixed-Probe Tracking of the Field Between Trolley Measurements . . . . . 71

3.4 Averaging over Muon Distribution . . . . . . . . . . . . . . . 72

3.5 Other Systematic Effects . . . . . . . . . . . . . . . . . . 72

3.6 Final Value of $\omega_{p} \ldots \ldots \ldots \ldots \ldots \ldots \ldots$

42001 Spin Precession Frequency Measurement $\quad 75$

4.1 Data Selection . . . . . . . . . . . . . . . 75

4.1 Run Selection . . . . . . . . . . . . . . . . . . . . . . . . 75

4.1 .2 Fill Selection . . . . . . . . . . . . . . 76

4.2 Construction of Decay Electron Time Spectra . . . . . . . . . . . . . 77

4.2 .1 Energy Calibration . . . . . . . . . . . . . . . 77

4.2.2 Determination of Lower Energy Threshold . . . . . . . . . . . . 78

$4.2 .3 \quad$ Histogram Filling . . . . . . . . . . . . . . . . . . . . . 80

4.2.4 Removing Pileup From the Data . . . . . . . . . . . . . . . 80

4.2.5 Energy Scale Changes . . . . . . . . . . . . . . . . 84

4.2.6 Beam Debunching and Fill Randomization . . . . . . . . . . . . 89

4.2 .7 Muon Losses . . . . . . . . . . . . . . . . . . . . . . . . . . 90

4.2.8 The Ratio Method . . . . . . . . . . . . . . . . . . 91

4.3 Fits to the Data . . . . . . . . . . . . . . . . 96

4.3 .1 CBO Parameters . . . . . . . . . . . . . . . 96

$4.3 .2 \quad$ Fit Procedure . . . . . . . . . . . . . . . . . . . . . 103

$4.3 .3 \quad$ Fit Results . . . . . . . . . . . . . . . . . . . . . 103

4.42001 Systematic Errors on $\omega_{a} \ldots \ldots \ldots \ldots \ldots$

4.4 .1 Energy Bin Analysis . . . . . . . . . . . . . . . . . . . 123

4.4 .2 Energy Scale Changes . . . . . . . . . . . . . . . . . . . . . . . . . . . . . . . . . 127

4.4 .3 Coherent Betatron Oscillations . . . . . . . . . . . . . . . 131

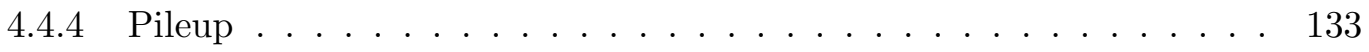

4.4 .5 Muon Losses . . . . . . . . . . . . . . . . . . 136

4.4.6 Changes in Beam Position . . . . . . . . . . . . . . . . . . . . 143

4.4 .7 Other Systematic Uncertainties . . . . . . . . . . . . . . . . . . . 144

4.5 Summary . . . . . . . . . . . . . . . . . . . . 152

$\begin{array}{llr}5 & \text { Conclusion } & 157\end{array}$

5.1 Determination of $a_{\mu^{-}} \ldots \ldots \ldots \ldots \ldots \ldots \ldots \ldots$

5.2 Discussion . . . . . . . . . . . . . . . . . . . 157

5.2 .1 Muon Electric Dipole Moment _. . . . . . . . . . . . . 157

5.2 .2 Limits on New Physics . . . . . . . . . . . . . . . . . . 159

5.3 Future Prospects . . . . . . . . . . . . . . . . . 161

$\begin{array}{ll}\text { A G2OFF Ntuple Structure } & 163\end{array}$ 
B The Ratio Method - Derivations 167

B.1 Simple 3-Parameter Function . . . . . . . . . . . . . . . . 167

B.2 Derivation of the Ratio Function with a Background Term . . . . . . . . 169

B.3 A General Derivation of the Ratio Function with CBO . . . . . . . . . . 170

B.4 Error Propagation . . . . . . . . . . . . . . . . . . . . . . 174 


\section{List of Figures}

$1.1 g=2$ and first order QED correction to $a_{\mu} \ldots \ldots \ldots \ldots$

1.2 Higher order QED Feynman diagrams. . . . . . . . . . . . . . . . 4

1.3 Weak interaction loops in $a_{l} \ldots \ldots \ldots \ldots \ldots$

1.4 Feynman diagram for the first order hadronic vacuum polarization contribution to the magnetic moment. . . . . . . . . . . . . 6

1.5 Feynman diagrams of $\tau$-decay into hadrons. . . . . . . . . . . . 8

$1.6 \sigma\left(e^{+} e^{-} \rightarrow\right.$ hadrons $)$ vs. energy squared, s. . . . . . . . . . . 10

1.7 Difference between $e^{+} e^{-}$and $\tau$ decay spectral functions. . . . . . . . . . . 10

1.8 Feynman diagram for the Hadronic light-by-light contribution. . . . . . . . 12

$2.1 n(y)$ and $A(y)$ of muon decay in the rest frame. . . . . . . . . . . 17

2.2 Top view of the AGS beamline. . . . . . . . . . . . . 20

2.3 Simulated distribution of muon spin. . . . . . . . . . . . . . . 21

2.4 Top view of the injection point of the beam into the $g$-2 storage ring. . . . . 23

2.5 Cross-section view of the $g$-2 superconducting inflector. . . . . . . . . . 23

2.6 Cross section view of the $g-2$ storage ring. . . . . . . . . . . . . . . . . . 24

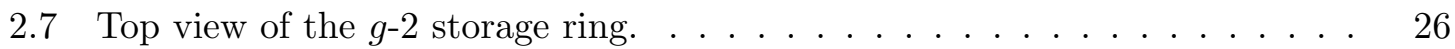

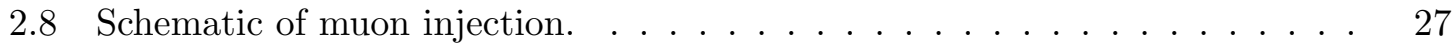

2.9 Circuit diagram of the "ideal" kicker electronics. . . . . . . . . . . . 28

2.10 Side view of a kicker module. . . . . . . . . . . . . . . . . 29

2.11 Cross-section view of the storage aperture in the kicker region. . . . . . . . 30

2.12 Quadrupole geometry. . . . . . . . . . . . . . . . . . . . 30

2.13 Top and cross-section views of the electrostatic quadrupoles. . . . . . . . . . 34

2.14 Tune plane in the region near the operating values of the $g$ - 2 storage ring. . 35

2.15 Illustration of coherent betatron motion. . . . . . . . . . . . . . 36

2.16 Schematic of the radial phase-space diagram of the beam. . . . . . . . . 37

2.17 Coordinate system used to derive vertical pitch correction to $\omega_{a}$. . . . . . 39

2.18 Time spectrum of decay electrons 6-12 $\mu$ s (left) and 36-42 $\mu$ s (right) after

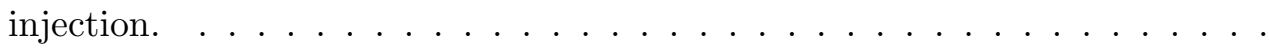

2.19 Equilibrium radial distributions obtained using two different fast-rotation

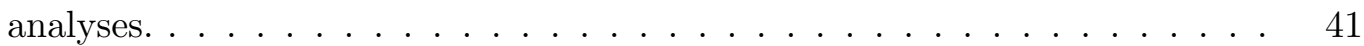

2.20 Sketch of the fiber beam monitors. . . . . . . . . . . . . . . . 42

2.21 Time and frequency spectra of a single fiber read-out. . . . . . . . . . . 43

2.22 Coordinate system used in g2track. . . . . . . . . . . . . . . . 44

2.23 Average kicker pulse shapes and T0 pulse shape, as read from WFDs. . . . 47 
2.24 Simulated kicker current pulse used in g2track. . . . . . . . . . . . . . . . . . 48

2.25 Injected muon radial and vertical distributions. . . . . . . . . . . . . . 49

2.26 Phase space distributions of the injected muons. . . . . . . . . . . . . 49

2.27 Phase-space diagram of $x$ vs. $x^{\prime}$ for a single muon in the g2track simulation. 50

2.28 Results from g2track in "FBM" mode. . . . . . . . . . . . . . . 51

2.29 Fraction of lost muons as a function of applied quadrupole high voltage. . . $\quad 52$

2.30 Time spectrum and azimuthal location of where muons are lost in g2track. $\quad 52$

2.31 Schematic of NMR process. . . . . . . . . . . . . . 54

2.32 A typical NMR probe used in this experiment. . . . . . . . . . . 55

2.33 The electronics of the pulsed NMR magnetometer. . . . . . . . . . . 56

2.34 Time (top) and Fourier frequency (bottom) spectra of the FID signal obtained from a single NMR probe. . . . . . . . . . . . . . 57

2.35 Schematic of the plunging probe used to calibrate the trolley probes. . . . . 57

2.36 Schematic of the standard calibration probe. . . . . . . . . . . . . 58

2.37 The NMR trolley used to map out the magnetic field inside the $g$ - 2 storage

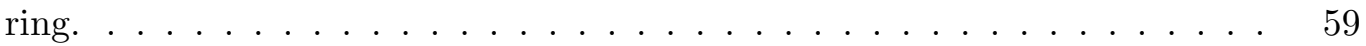

2.38 Top view of location of a single detector used in the experiment. . . . . . . 59

2.39 Schematic drawing and photo of one of the 24 electromagnetic calorimeters used in the experiment. . . . . . . . . . . . . . . . 61

2.40 Schematic of the DAQ system in acquisition mode. . . . . . . . . . . . 62

2.41 Block diagram of the DAQ system. . . . . . . . . . . . . . . . 64

2.42 Simplified block diagram of the ntuple data structure. . . . . . . . . . . . 66

2.43 Brookhaven E821 measurements of $a_{\mu}$ as a function of time. . . . . . . 68

3.1 Relative fluctuations of the center trolley probe measurement of the magnetic field around the storage ring. . . . . . . . . . . . 70

3.2 Contour plot of the dipole magnetic field, averaged over azimuth, in the storage aperture. . . . . . . . . . . . . . . . . 71

4.1 Fill selection as a function of run number. . . . . . . . . . . . . 77

4.2 Energy calibration on a run-by-run basis for detectors 3, 10, 17 and 23. . . 78

$4.3 N(E), A(E)$ and $N A^{2}(E)$ for detector $1 \ldots \ldots \ldots \ldots \ldots$

4.4 Energy threshold values at the peak of $N A^{2}$ vs. $E_{t} \ldots \ldots \ldots$. . . . . 80

4.5 Method of pileup construction. . . . . . . . . . . . . . . . 81

4.6 Energy spectra for a few of the individual detectors. . . . . . . . . . . 83

4.7 Energy at which the number of pileup events crosses zero as a function of detector. . . . . . . . . . . . . . . . 83

4.8 Constructed pileup time spectra for detector 1 using different energy cuts. . 84

4.9 Average energy vs. time after injection. . . . . . . . . . . . . 85

4.10 Determination of relationship between gain and change in average energy. . $\quad 86$

4.11 Results of energy-scale change correction. . . . . . . . . . . . . . 87

4.12 Comparison of fit $\chi^{2} /$ d.o.f. vs. fit time between between un-corrected and gain-corrected data. . . . . . . . . . . . . . . .

4.13 Comparison of fit asymmetry vs. fit time between uncorrected data sets and gain-corrected data sets. . . . . . . . . . . . . . . 88 
4.14 Comparison of time and frequency spectra of fill-randomized data and nonrandomized data. . . . . . . . . . . . . . . . . .

4.15 Number of three-fold coincidences (3FCs) as a function of time in the low-n 2001 data. . . . . . . . . . . . . . . . . . . . . . . . 90

4.16 Shifting the time spectra in the ratio method. . . . . . . . . . . . . 92

4.17 Difference and sums of phase-shifted time spectra. . . . . . . . . . . . 93

4.18 Original $N(t)$ spectrum and ratio function, both produced using all 2001 data. 94

4.19 Results of fits to simulated data using the five-parameter function and the three-parameter ratio function. . . . . . . . . . . . . . 95

4.20 Schematic of muon decay inside the storage region. . . . . . . . . . . 96

4.21 FFT of the residuals of a five parameter fit of the low-n electron decay time spectrum at $180 \mu \mathrm{s}$ after injection. . . . . . . . . . . . . 98

4.22 FFT of the residuals of a five parameter fit of the high-n electron decay time spectrum at $180 \mu \mathrm{s}$ after injection. . . . . . . . . . . . . . . 99

4.23 Determination of the CBO envelope. . . . . . . . . . . . . . . 100

4.24 Distributions of CBO lifetime and frequency. . . . . . . . . . . . . . 101

4.25 Effect of the kicker and quadrupole plates on electrons passing through on the way to a detector. . . . . . . . . . . . . . .

$4.26 R$ vs. Detector for the low- and high-n data sets using the three-parameter ratio function. . . . . . . . . . . . . . . . . . . 104

4.27 Fit results using the three-parameter ratio function. . . . . . . . . . . 105

$4.28 R$ vs. Detector for the low- and high-n data sets using the five-parameter ratio function. . . . . . . . . . . . . . . . . 107

4.29 Fit results using the five-parameter ratio function. . . . . . . . . . . 108

4.30 CBO amplitude (top) and phase (bottom) vs. detector for the five parameter ratio fit. . . . . . . . . . . . . . . . . . . 109

$4.31 \mathrm{R}$ vs. Detector for the seven parameter ratio fit. . . . . . . . . . . . . 110

4.32 Fit results using the seven-parameter ratio function. . . . . . . . . . . . . 111

$4.33 N_{\mathrm{CBO}}$ amplitudes and phases vs. Detector for the seven parameter ratio fit. 112

$4.34 A_{\mathrm{CBO}}$ term amplitudes and phases vs. Detector for the seven parameter ratio fit. . . . . . . . . . . . . . . . . . . . 112

$4.35 \mathrm{R}$ vs. Detector for the nine parameter fit. . . . . . . . . . . . . . . 113

4.36 Fit results using the nine-parameter ratio function. . . . . . . . . . . . . 114

$4.37 N_{\mathrm{CBO}}$ term amplitudes and phases vs. Detector for the nine parameter fit. . 115

$4.38 A_{\mathrm{CBO}}$ term amplitudes and phases vs. Detector for the nine parameter fit. . 115

$4.39 \phi_{\mathrm{CBO}}$ term amplitudes and phases vs. Detector for the nine parameter fit. . 116

4.40 Fit result comparison of $R$ vs. Det. for the low-n data set using different fit start times for each detector. . . . . . . . . . . . . . . . 117

4.41 Fit result comparison of $R$ vs. Det. for the high-n data set using different fit start times for each detector. . . . . . . . . . . . . .

4.42 Difference between first-half and second-half of the ring of $R$ vs. fit time for various fit functions. . . . . . . . . . . . . . . . . . 120

4.43 Difference between various fit functions of $R$ vs. fit time plots of detector 27. 120

$4.44 R$ vs. Detector for the low-n (Golden and Silver+Bronze) and high-n data sets using different start times for each detector. . . . . . . . . . . . . . 124 
4.45 Various parameters as functions of energy bin obtained from fits to the low-n and high-n data sets. . . . . . . . . . . . . . . . . . . 125

4.46 Comparison between G2GEANT simulated data and actual data. . . . . . . 126

$4.47 R$ vs. Energy bin for the low-n and high-n data sets. . . . . . . . . . . . . 127

4.48 Difference of $R$ vs. fit start time plots between uncorrected and gain-corrected data. . . . . . . . . . . . . . . . . . 128

4.49 Distributions of $\delta R_{\mathrm{ESC}} \ldots \ldots \ldots \ldots \ldots \ldots \ldots \ldots \ldots$

4.50 The varying WFD island length results in another ESC-like systematic uncertainty on $\omega_{a} \ldots \ldots \ldots \ldots \ldots \ldots \ldots$

4.51 Distributions of CBO uncertainties determined for each of the 23 detectors at their corresponding fit start times. . . . . . . . . . . . . . 132

4.52 Estimation of $\delta R_{\alpha_{P U S}}$, the pileup construction efficiency systematic error. . 134

4.53 Determination of $d R / d \phi_{P U} \ldots \ldots \ldots \ldots \ldots \ldots \ldots \ldots$

4.54 Muon loss time spectrum used to determine $\delta R_{\text {loss }} \ldots \ldots \ldots \ldots \ldots \ldots$

4.55 Effect of muon losses on $R$ vs. fit start time with the ratio method. . . . . . 138

4.56 Effect on $R$ vs. fit start time of muon losses assuming a $0.6 \%$ total loss and different average $g-2$ phases and asymmetries. . . . . . . . . . . . . 139

4.57 Comparison of initial $g$-2 phases of muons that are lost and stored in gtrack. 139

4.58 Average $g-2$ phase as a function of radial and vertical position in the storage aperture, determined from G2GEANT. . . . . . . . . . . . . . . . 140

4.59 Average $g$-2 phase of stored (top) and lost (bottom) muons in g2track as a function of time. . . . . . . . . . . . . . . .

4.60 Changes in $R$ vs. fit start time for such differences between the lost and stored muon $g-2$ phases as were found from g2track simulations. . . . . . . 143

4.61 Vertical oscillations as seen by the FSDs by looking at the differences between tiles 2 and 4 as a function of time. . . . . . . . . . . . . 146

4.62 FFT of FSD "tile 2 - tile 4" time spectra. . . . . . . . . . . . . . 147

4.63 Effect on the fit values of $R$ when neglecting the various other betatron oscillation terms. . . . . . . . . . . . . . . . . . 148

4.64 Flashlet time and frequency spectra, all detector data summed together, for both data sets. . . . . . . . . . . . . . . . . . . 149

4.65 Flashlet energy spectrum and distribution around the ring. . . . . . . . 150

4.66 Comparison of the five $\omega_{a}$ analyses. . . . . . . . . . . . . 153

5.1 Comparison of the most current experimental and theoretical values for $a_{\mu}$. $\quad 158$

5.2 Allowed regions for masses of the two lightest supersymmetric partner particles. 160

B.1 The "fast factor" as a function of time after injection. . . . . . . . . 175 


\section{List of Tables}

2.1 The multipole terms of the electric field used to focus the beam. . . . . . . 33

3.1 Systematic error table for the $\omega_{p}$ measurement. . . . . . . . . . . . . . . 74

4.1 Results of Gaussian fits to the four main peaks in the FFT spectrum of residuals to fits at late times. . . . . . . . . . . . . . . 97

4.2 Fit start times for the individual detectors. . . . . . . . . . . . . . . 117

4.3 Summary of fit parameter status in each ratio fit. . . . . . . . . . . . 119

4.4 Brief summary of the current results from the various functions fit to the two data sets. . . . . . . . . . . . . . . . . . . . 121

4.5 Correlation Matrix of the 9 fit parameters of the full physics function for the low-n data set. . . . . . . . . . . . . . . . . . . . . . . 122

4.6 Comparison of the means and widths of the radial distributions obtained by the two fast rotation analyses. . . . . . . . . . . . . . . 151

4.7 Values of the fit parameters for the nine-parameter ratio fit. . . . . . . . . 153

4.8 Summary of systematic errors for the various individual and combined data sets. . . . . . . . . . . . . . . . . . . 154

A.1 GENERAL data block of the G2OFF decay electron ntuple data structure. . . . 163

A.2 PULSES, PULSESL and SLIMDATA data blocks of the G2OFF decay electron ntuple data structure. . . . . . . . . . . . . . . . . . . . 164

A.3 TOPULSE, LPULSE and CALMTDC data blocks of the G2OFF decay electron ntuple data structure. . . . . . . . . . . . . . . . . . 165

A.4 KICKER, PSD2, PSDT, FSDPULSE, PSD2PC and PSDA data blocks of the G2OFF decay electron ntuple data structure. . . . . . . . . . . . . . . 166 



\section{List of Acronyms}

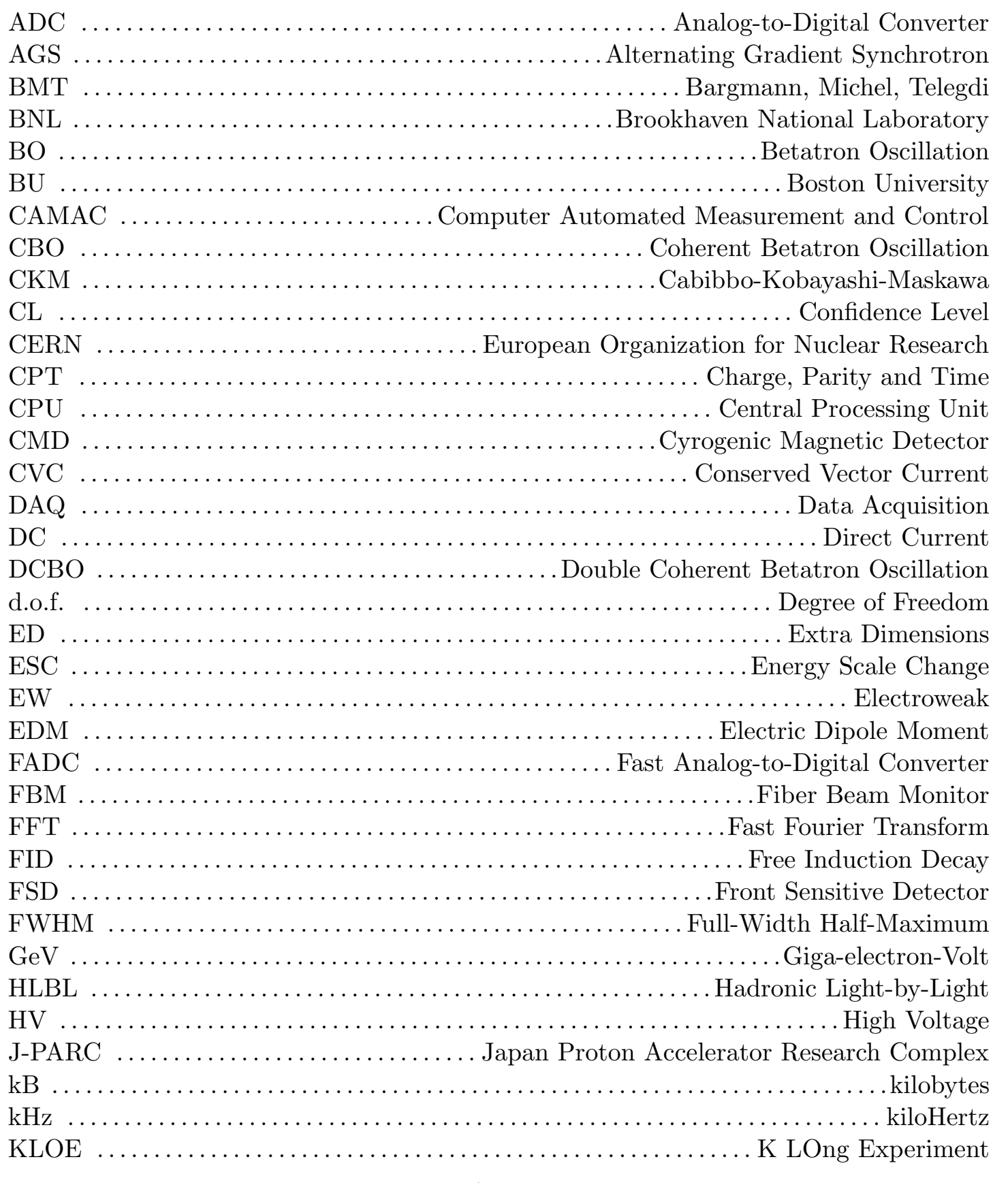



. nanosecond parts per billion

PU Supersymmetry 


\section{Chapter 1}

\section{Introduction}

Measurements of anomalous magnetic moments have provided great insights within the field of subatomic particle physics. The measurement of the proton magnetic moment was one of the first indications of substructure in the particle, a major piece of the puzzle that led to the development of quantum chromodynamics (QCD). The ultra-precise determination of the anomalous magnetic moment of the electron provides the best determination of the quantum electrodynamics (QED) coupling constant, $\alpha$, at $q^{2}=0$. The present measurements of the anomalous magnetic moment of the muon provide a unique and stringent test of the electroweak theory in the Standard Model of particle physics, and are also a sensitive test of physics beyond the Standard Model. The anomalous magnetic moment of the positive muon has already been measured to about 0.7 parts per million (ppm) at the Alternating Gradient Synchrotron at the Brookhaven National Laboratory, the results of which have provoked much discussion within the physics community and a re-evaluation of the theory. This dissertation will discuss both the principle of and the analysis techniques used in the measurement of the anomalous magnetic moment of the negative muon to about $0.7 \mathrm{ppm}$, and will compare the final results with the theory.

\subsection{Background on the Anomalous Magnetic Moment}

\subsubsection{Some Definitions}

In classical quantum mechanics, the gyromagnetic ratio, $g_{s}$, of a spin $\frac{1}{2}$ particle is given by ${ }^{1}$

$$
\overrightarrow{\mu_{s}}=g_{s} \frac{e}{2 m} \vec{S}
$$

where $\mu_{s}$ and $\vec{S}$ are the magnetic dipole moment and the spin vector of the particle respectively.

The first observation of quantized atomic magnetic moments was made by Stern and Gerlach in the 1922[1] experiment where a flat beam of silver atoms was passed through a non-homogeneous magnetic field and the beam was observed to split into two. Classical theory predicts a continuous spread of the beam. The observation of the beam splitting was first explained by Goudsmit and Uhlenbeck [2] as a result of electron spin quantization.

\footnotetext{
${ }^{1}$ Unless otherwise noted, we use natural units where $\mathrm{c}=\hbar=1$.
} 
Similar experiments were later conducted using hydrogen and other atoms (eg: [3]). Dirac's quantization of Einstein's relativistic energy equation [4] in 1928 showed that quantized spin arises from Lorentz invariance and predicted that for a spin $1 / 2$ point particle such as the electron, $g_{s}=2$.

However, due to various effects, $g_{s}$ is never equal to 2 for any spin $1 / 2$ particle. Estermann and Stern [5] were the first to find that the proton's gyromagnetic ratio is very different from two $\left(g_{p} \simeq 5.59\right)$. This difference was one of the first indications that the proton is not a point particle but has substructure (quarks and gluons).

Electrons and muons are considered elementary point particles, since no signs of substructure at the level of $10^{-18} \mathrm{~cm}$ for these particles has been found. However, because of quantum fluctuations of the vacuum, their gyromagnetic ratios also differ from two. The sum of the quantum contributions to the magnetic moment is expressed simply as

$$
a \equiv \frac{g-2}{2}
$$

which is known as the anomalous magnetic moment.

In the Standard Model of particle physics, the theoretical values of the anomalous magnetic moment of the electron and muon are traditionally written as

$$
a_{l}^{\mathrm{SM}}=a_{l}^{\mathrm{QED}}+a_{l}^{\mathrm{Weak}}+a_{l}^{\mathrm{Had}}
$$

where $l$ is the lepton of interest and the contributions to the anomaly are grouped according to the fundamental interactions involved: quantum electrodynamical interactions (QED), weak interactions (Weak), and hadronic, or quantum chromodynamical, interactions (Had).

\subsubsection{The Electron $g-2$}

The development of the theory of QED had very close ties to the anomalous magnetic moment of the electron. In 1948 Kusch and Foley [6] measured the gyromagnetic ratio of the electron to be $g_{e}=2(1.00119 \pm 0.00005)$. This measurement, along with the Lamb Shift, motivated the first calculation of $a_{e}$ by Schwinger in 1947 [7]. Schwinger calculated the first-order QED contribution to $a_{e}$, shown in Fig. 1.1(b). At the time, QED was in the early stages of its development, beset by divergence difficulties. Schwinger's tour-de-force calculation of $a_{e}=\alpha / 2 \pi \simeq 0.00118$, where $\alpha \simeq 1 / 137$ is the fine structure constant, agreed wonderfully with the experimentally determined value from Kusch and Foley.

QED has since been fully developed, and one may, in principle, calculate the QED contribution to $a_{e}$ to any desired precision. However, due to computational limits (that is, the need to calculate an exponentially increasing number of Feynman diagrams from one order of $\alpha / \pi$ to the next), the theoretical calculation of $a_{e}^{\mathrm{QED}}$ has been made to fourth order in $\alpha / \pi[8]$ (see Figs. 1.1 and 1.2 for examples of the interactions involved):

$$
a_{e}^{\mathrm{SM}}=C_{1} \frac{\alpha}{\pi}+C_{2}\left(\frac{\alpha}{\pi}\right)^{2}+C_{3}\left(\frac{\alpha}{\pi}\right)^{3}+C_{4}\left(\frac{\alpha}{\pi}\right)^{4}+1.70(3) \times 10^{-12}
$$

where the first four terms are QED interactions and the very last term is the contribution from weak and hadronic interactions. The first three QED terms are known analytically [9] 


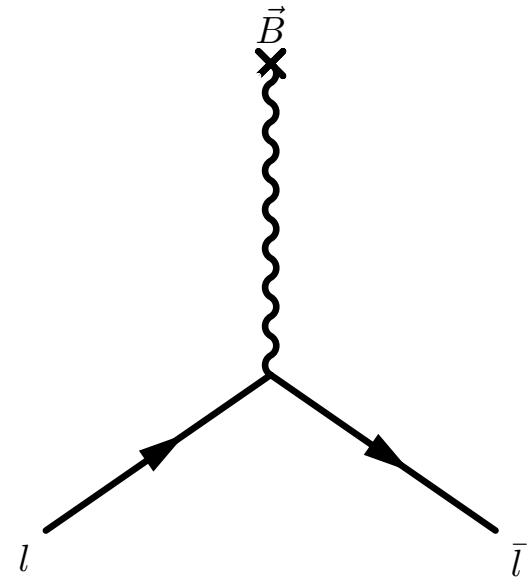

(a) $g=2$ exactly

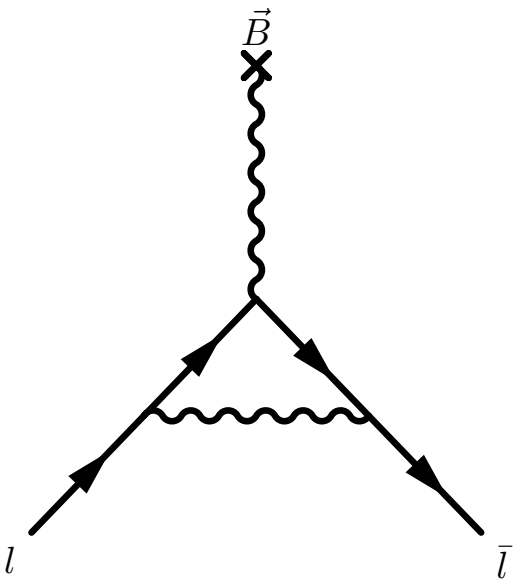

(b) First order QED correction $(=\alpha / 2 \pi)$

Figure 1.1: QED Feynman diagrams. $l$ represents an incoming lepton (either electron or muon).

where

$$
C_{1}=\frac{1}{2}, \quad C_{2}=-0.32847844400, \quad C_{3}=1.181234017
$$

and the fourth term has been computed to be [10]

$$
C_{4}=1.7502(384)
$$

Now, $a_{e}$ is one of the most precisely measured physical constants, with a relative uncertainty of 4 parts per billion (ppb)[11]:

$$
a_{e^{-}}^{\exp }=1159652188.4(4.3) \times 10^{-12}
$$

and

$$
a_{e^{+}}^{\exp }=1159652187.9(4.3) \times 10^{-12}
$$

Using the experimental value of $a_{e}$, and assuming all experimental and theoretical errors are under control, the best determination of the fine structure constant at $q^{2}=0[10]$ is:

$$
\alpha_{a_{e}}^{-1}=137.03599875(52)
$$

The next-best determination of the fine structure constant $\alpha$ comes from the measurement of the quantum Hall effect[12]

$$
\alpha_{\mathrm{QHE}}^{-1}=137.03600300(270)
$$

over five times less accurate than $\alpha_{a_{e}}$.

Since the weak and hadronic interaction contributions to $a_{e}$ are smaller than the exper- 


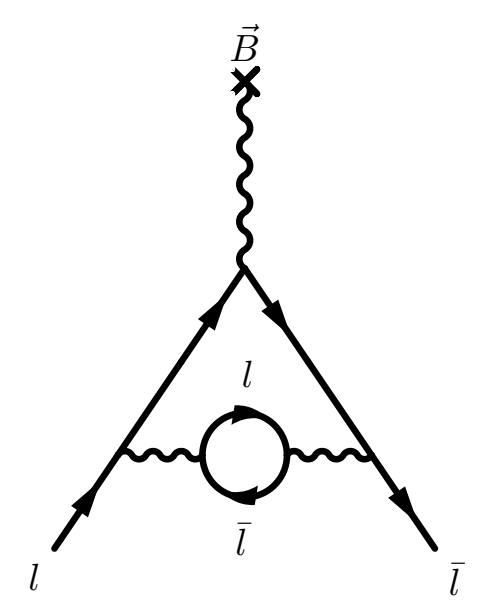

(a) Example of a $2^{\text {nd }}$ order QED correction, $O\left((\alpha / \pi)^{2}\right)$

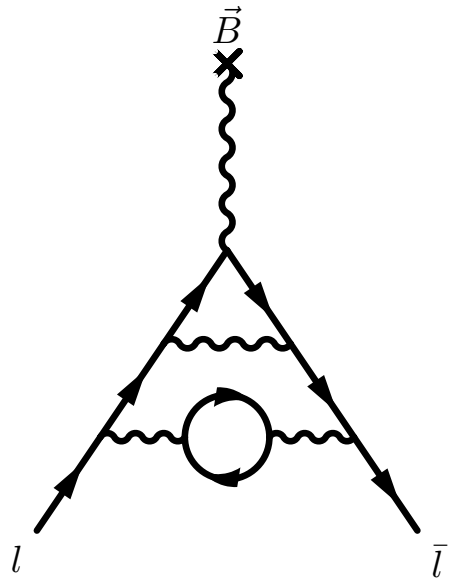

(b) Example of a $3^{r d}$
order QED
$O\left((\alpha / \pi)^{3}\right)$

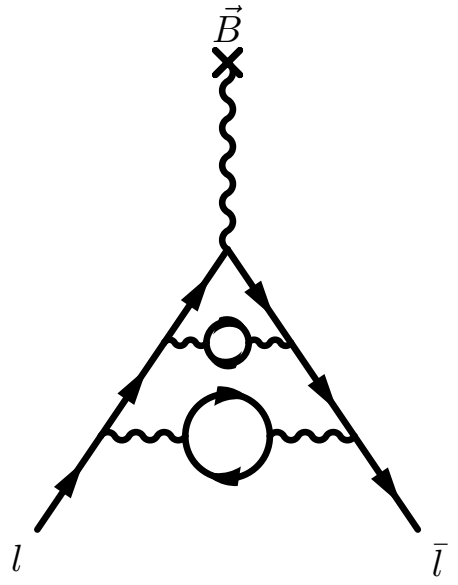

(c) Example of a $4^{\text {th }}$ order QED correction, $\left.O\left((\alpha / \pi)^{4}\right)\right)$

Figure 1.2: Higher order QED Feynman diagrams. $l$ represents an incoming lepton (either electron or muon).

imental error, $a_{e}$ is not very useful for probing higher energy scale physics. On the other hand, both the hadronic and weak interaction contributions to $a_{l}$ are proportional to $m_{l}^{2}$, where $m_{l}$ is the mass of the lepton. Since the muon is approximately 200 times more massive than the electron, $a_{\mu}^{\text {Weak }}$ and $a_{\mu}^{\text {Had }}$ are $\left(m_{\mu} / m_{e}\right)^{2} \sim 4 \times 10^{4}$ times larger for $a_{\mu}$ than for $a_{e}$. Therefore the muon anomalous magnetic moment is much more sensitive to larger energy scale physics that that of the electron, and it is for this reason that $a_{\mu}$ is such an interesting property to measure.

\subsection{QED Contributions to $a_{\mu}$}

The QED contribution to $a_{\mu}$ has been calculated either analytically or by numerical methods through 5 loops [10]

$$
a_{\mu}^{\mathrm{QED}}=C_{1} \frac{\alpha}{\pi}+C_{2}\left(\frac{\alpha}{\pi}\right)^{2}+C_{3}\left(\frac{\alpha}{\pi}\right)^{3}+C_{4}\left(\frac{\alpha}{\pi}\right)^{4}+C_{5}\left(\frac{\alpha}{\pi}\right)^{5}
$$

where again,

$$
C_{1}=\frac{1}{2}
$$

and

$$
\begin{gathered}
C_{2}=0.765857399(45), \quad C_{3}=24.0505095(23), \\
C_{4}=125.08(41), \quad C_{5}=930(170)
\end{gathered}
$$




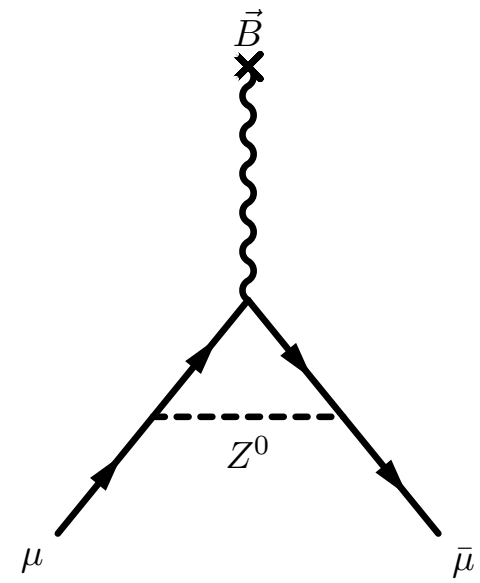

(a) First order weak correction

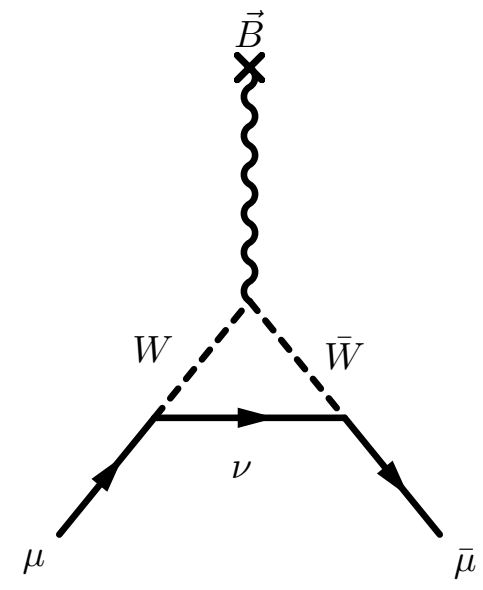

(b) First order weak correction

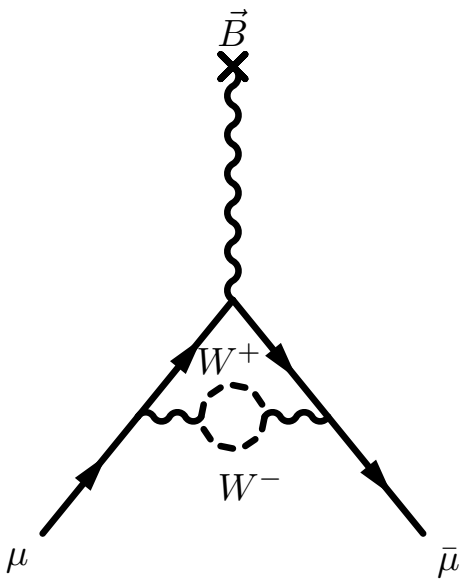

(c) Example of a $2^{\text {nd }}$ order weak correction

Figure 1.3: Weak interaction loops in $a_{l}$.

The large increase in the coefficients of the expansion arises from the electron loop $\left(\ln \left(m_{\mu} / m_{e}\right) \simeq\right.$ 5.3) terms and the very large contribution of the light-by-light electron loop in $C_{3}$ (nearly $88 \%$ of the entire value of $\left.C_{3}\right)$ [8].

Using $\alpha^{-1}\left(a_{e}\right)$ (Eq. 1.9), the theoretical value of $a_{\mu}^{\mathrm{QED}}$ is found to be [10]

$$
a_{\mu}^{\mathrm{QED}}=116584703.5(2.8) \times 10^{-11}
$$

which gives a relative uncertainty at the level of $24 \mathrm{ppb}$.

\subsection{Weak Contributions to $a_{\mu}$}

The smallest contribution to $a_{\mu}$ arises from weak interaction loops, and only a calculation to second order has been necessary to test the theory at the present experimental limits (existing or proposed). Examples of some of the weak interaction Feynman diagrams are shown in Fig. 1.3. The first order correction is $[13,14]$

$$
a_{\mu}^{\mathrm{Weak}}(1)=\frac{5 G_{\mu} m_{\mu}^{2}}{24 \sqrt{2} \pi^{2}}\left[1+\frac{\left(1-4 \sin ^{2} \theta_{w}\right)^{2}}{5}+\mathcal{O}\left(\frac{m_{\mu}}{M}\right)^{2}\right]
$$

where $G_{\mu}$ is the Fermi constant, $\sin ^{2} \theta_{w}=1-m_{W}^{2} / m_{Z}^{2}$ is the weak mixing angle, and $M=m_{W}$ or $m_{\text {Higgs }}$. Plugging in the numbers, one finds [15]

$$
a_{\mu}^{\mathrm{Weak}}(1) \simeq 195 \times 10^{-11}
$$

The presence of $\ln \left(m_{Z}^{2} / m_{\mu}^{2}\right) \simeq 13.5$ terms in the second order electroweak correction results in a relatively large (with respect to the first order) contribution to $a_{\mu}$ [16]. The full two-loop calculation, which includes low-energy hadronic electroweak loops and assumes 


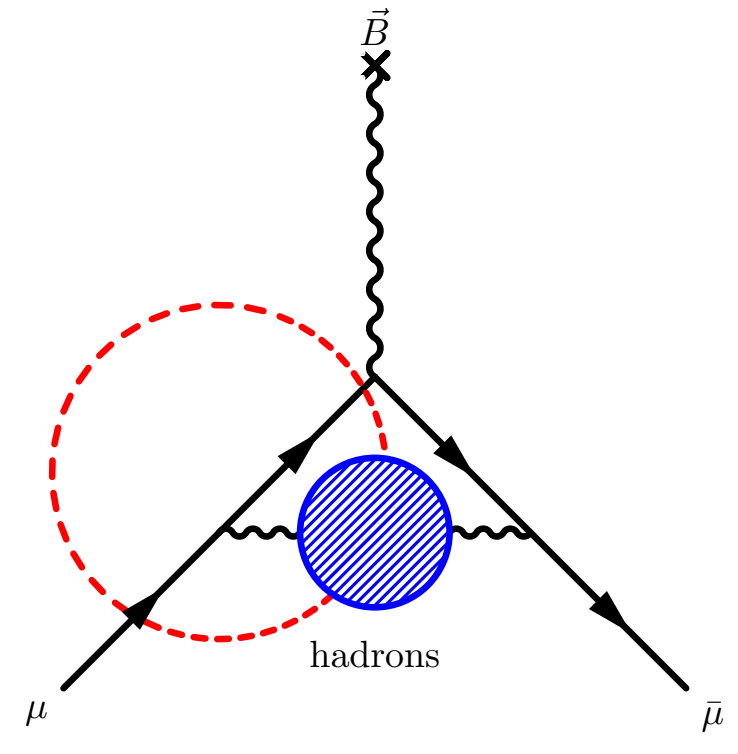

(a)

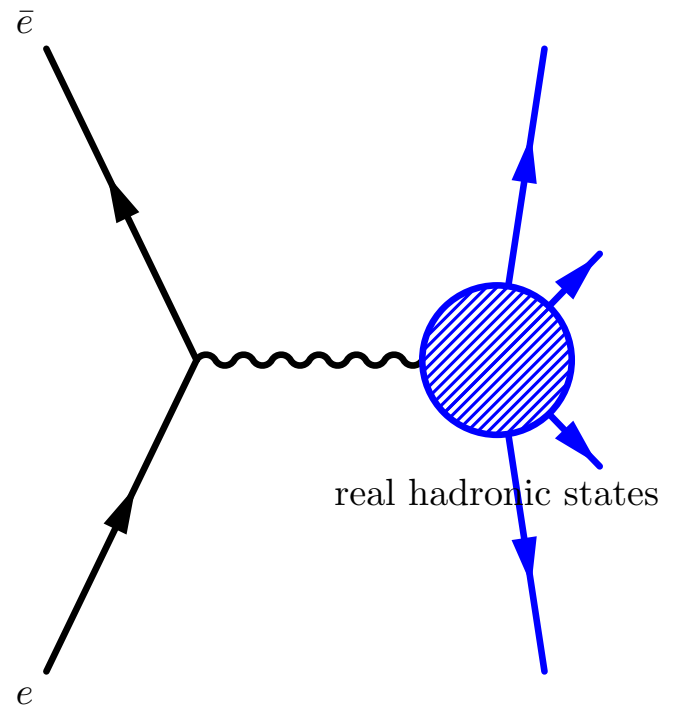

(b)

Figure 1.4: Feynman diagram for the first order hadronic vacuum polarization contribution to the magnetic moment. Splitting diagram 1.4(a) in "half" and replacing $\mu$ with $e$, one can relate $e^{+} e^{-} \rightarrow$ hadrons via the dispersion relation in Eq. 1.21

$m_{\text {Higgs }} \simeq 150 \mathrm{GeV}$, gives $[15]$

$$
a_{\mu}^{\mathrm{Weak}}(2)=-41(3) \times 10^{-11}
$$

where the error arises from uncertainties in the hadronic electroweak loop, the Higgs boson mass, and higher-order uncertainties [15].

\subsection{Hadronic Contributions to $a_{\mu}$}

Although the hadronic loops are the second largest contributor to $a_{\mu}$, they are by far the largest contributor to the theoretical uncertainty of $a_{\mu}$. As will be explained, the evaluation of the first-order hadronic contribution, $a_{\mu}^{\mathrm{Had}}(1)$, relies on experimental data. Therefore the uncertainty, $\delta a_{\mu}^{\mathrm{Had}}(1)$ is limited by the experimental uncertainties (statistical and systematic). Furthermore, calculations of the higher-order hadronic light-by-light contribution are model-dependent and are therefore only estimates with rather large uncertainties $(50 \%$ of the estimated value).

\subsubsection{First order vacuum polarization contribution}

The first order hadronic contribution to the anomaly, $a_{\mu}^{\mathrm{Had}}(1)$, results from vacuum polarization, as illustrated in Fig. 1.4(a). At $q^{2}=0$ the hadronic terms cannot be calculated from 
first principles in $\mathrm{QCD}^{2}$. We therefore use another approach to evaluate the contribution.

Since the hadronic vacuum-polarization tensor, or hadronic spectral function, $\Pi^{H}(s)$, is analytic, we may write the dispersion relation [17]

$$
a_{\mu}^{\mathrm{Had}}(1)=\frac{1}{\pi} \int_{0}^{\infty} \frac{d s}{s} \quad K(s) \quad \operatorname{Im} \Pi^{\mathrm{H}}(s)
$$

where $\operatorname{Im} \Pi^{H}(s)$ is the imaginary part of the hadronic spectral function and

$$
K(s)=\left(\frac{\alpha}{\pi}\right) \int_{0}^{1} d z \frac{z^{2}(1-z)}{z^{2}+\frac{s}{m_{\mu}^{2}}(1-z)}
$$

is a kinematic factor. The optical theorem, which originates from the unitarity of the scattering matrix, relates the imaginary part of the forward elastic scattering amplitude of an interaction to the total cross-section:

$$
\sigma^{0}\left(e^{+} e^{-} \rightarrow \text { hadrons }\right)(s)=\frac{4 \pi \alpha(s)}{s} \operatorname{Im}^{H}(s)
$$

In practice, the Born cross section $\sigma^{0}\left(e^{+} e^{-} \rightarrow\right.$ hadrons $)(s)$ may not be measured directly. Rather, the total cross section, $\sigma\left(e^{+} e^{-} \rightarrow\right.$ hadrons $)(s)$ is determined experimentally and must be corrected for initial state radiation. One wishes to use the Born cross section in the first-order calculation of $a_{\mu}^{\mathrm{Had}}$ in order to avoid double-counting some of the higher-order contributions.

Inserting Eq. 1.20 into Eq. 1.18 we find

$$
a_{\mu}^{\operatorname{Had}}(1)=\frac{1}{4 \alpha \pi^{2}} \int_{4 m_{\pi}^{2}}^{\infty} d s K(s) \sigma^{0}\left(e^{+} e^{-} \rightarrow \text { hadrons }\right)(s)
$$

For $s \geq 4 m_{\mu}^{2}, K(s)$ may be parameterized as [18]

$$
\begin{aligned}
K(s)= & x^{2}\left(1-\frac{x^{2}}{2}\right)+(1+x)^{2}\left(1+\frac{1}{x^{2}}\right)\left[\ln (1+x)-x+\frac{x^{2}}{2}\right]+ \\
& \frac{1+x}{1-x} x^{2} \ln x
\end{aligned}
$$

and

$$
x=\frac{1-\sqrt{\left(1-4 m_{\mu}^{2} / s\right)}}{1+\sqrt{\left(1-4 m_{\mu}^{2} / s\right)}}
$$

Fig. 1.4(b) illustrates the basic concept of this approach: the vacuum polarization in Fig. 1.4(a) is "split" in half (inside the circle) by the dispersion relation, and the virtual hadronic states are related to real hadronic final states of the $e^{+} e^{-}$interaction.

Since the leading-order behavior of $K(s)$ goes as $1 / s$, the low-energy region of $\sigma^{0}\left(e^{+} e^{-} \rightarrow\right.$ hadrons $)(s)$ is weighted more heavily than higher energies. Also, the low-energy region of $\sigma^{0}\left(e^{+} e^{-} \rightarrow\right.$ hadrons $)(s)$ is dominated by the $\rho$ and $\omega$ resonances. Therefore, nearly $92 \%$ of

\footnotetext{
${ }^{2}$ Thom Blum has recently begun work on a lattice-based calculation.
} 


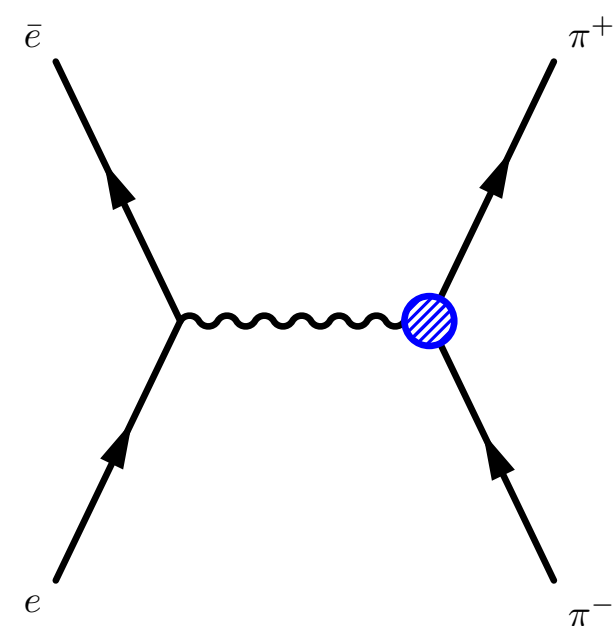

(a)

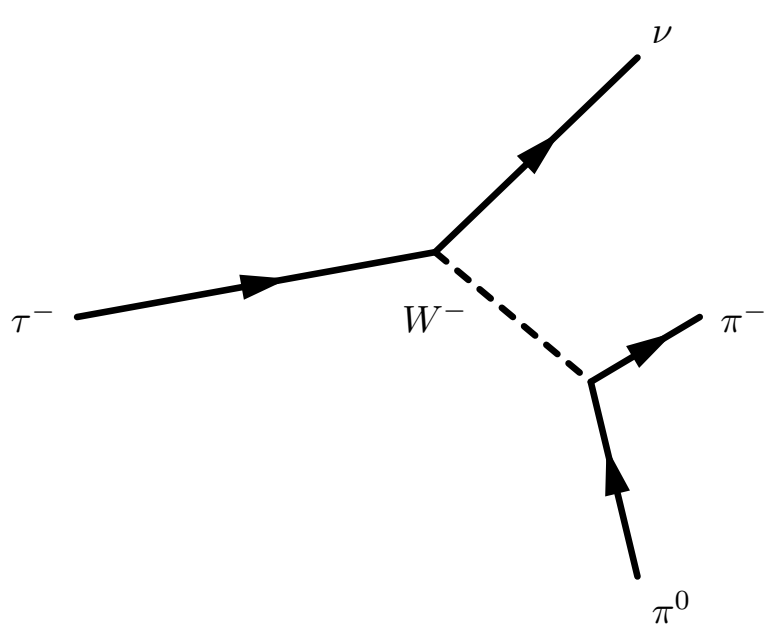

(b)

Figure 1.5: Via the CVC hypothesis, $\tau \rightarrow$ hadrons may be related to $\sigma\left(e^{+} e^{-} \rightarrow\right.$ hadrons $)$.

the value of Eq. 1.21 comes from the energy region $2 m_{\pi}<\sqrt{s}<1.8 \mathrm{GeV}$.

Many experiments around the world have determined the hadronic cross sections at various energies. Below $1.4 \mathrm{GeV}$, the CMD-2 experiment at Novosibirsk currently offers the most precise data for $e^{+} e^{-} \rightarrow$ hadrons, where the pion form factor has been measured with a systematic of $0.6 \%$. Therefore the data from CMD-2 dominate the measured low-energy spectrum (see, for example, Fig. 1.6). In order to obtain the bare cross sections from the data, corrections must be made for initial and final state radiation, electron-vertex loop contributions and vacuum polarization effects in the photon propagator[19]. Some of these corrections are quite sizeable, and have a large impact on the final determination of $a_{\mu}^{\mathrm{Had}}$. In fact, for CMD-2 the dominant contribution of the systematic uncertainty comes from the uncertainty of the radiative corrections, at $0.4 \%$. All other uncertainties, from event separation, fiducial volume corrections, detection efficiency corrections, pion loss corrections and beam energy determination are $0.2 \%$ or less.

Since the high hadron multiplicity in the energy region above $2.0 \mathrm{GeV}$ makes the measurement of the exclusive cross sections impractical, experiments done in this energy region measure the inclusive cross section $\sigma\left(e^{+} e^{-} \rightarrow\right.$ hadrons $)(s)$, and $a_{\mu}^{\mathrm{Had}}(1)$ is rewritten as

$$
a_{\mu}^{\operatorname{Had}}(1)=\frac{\alpha^{2}(0)}{3 \pi^{2}} \int_{4 m_{\pi}^{2}}^{\infty} d s \frac{K(s) R(s)}{s}
$$

where

$$
R(s)=\frac{\sigma\left(e^{+} e^{-} \rightarrow \text { hadrons }\right)(s)}{\sigma\left(e^{+} e^{-} \rightarrow \mu^{+} \mu^{-}\right)(s)}
$$

In the energy region between 2 and $5 \mathrm{GeV}$, measurements of $R$ (for example by such collaborations as BES in Beijing) are used to calculate $a_{\mu}^{\mathrm{Had}}(1)$. Above $5 \mathrm{GeV}$, perturbative QCD calculations are used.

As pointed out in [20], in the low-energy region, one may also use $\tau$-decay data to determine precisely the pionic cross sections using the conserved vector current (CVC) 
hypothesis. The CVC hypothesis is based on the idea that the weak coupling to the pion is related to the photon coupling by replacing $e$ (in the QED vertex) by $\sqrt{2} g \cos \theta_{c}$ [21], where

$$
\left(\frac{g}{M_{W}}\right)^{2}=\frac{G_{\mu}}{\sqrt{2}}
$$

and $\theta_{c}$ is the Cabbibo angle. If one assumes isospin symmetry, then the CVC hypothesis may be used to relate the two-pion production process $e^{+} e^{-} \rightarrow \pi^{+} \pi^{-}$to the decay $\tau^{-} \rightarrow \nu_{\tau} \pi^{-} \pi^{0}$ (shown in Fig. 1.5(b)) as well as the four-pion production process (eg: $\tau^{-} \rightarrow \nu_{\tau} 2 \pi^{-} \pi^{+} \pi^{0}$ ) by the relationships $[21,22]$

$$
\begin{gathered}
\sigma^{I=1}\left(e^{+} e^{-} \rightarrow \pi^{+} \pi^{-}\right)=\frac{4 \pi \alpha^{2}}{s} v_{\pi^{-} \pi^{0}} \\
\sigma^{I=1}\left(e^{+} e^{-} \rightarrow \pi^{+} \pi^{-}+\pi^{+} \pi^{-}\right)=\frac{8 \pi \alpha^{2}}{s} v_{\pi^{-} 3 \pi^{0}} \\
\sigma^{I=1}\left(e^{+} e^{-} \rightarrow \pi^{+} \pi^{-} 2 \pi^{0}\right)=\frac{4 \pi \alpha^{2}}{s}\left[v_{2 \pi^{-} \pi^{+} \pi^{0}}-v_{\pi^{-}} 3 \pi^{0}\right]
\end{gathered}
$$

$v_{V}(s)$ is the $\tau$ spectral function for a given vector hadronic state $V$, defined by

$$
v_{V}(s) \equiv \frac{m_{\tau}^{2}}{6\left|V_{u d}\right|^{2} S_{E W}} \frac{B\left(\tau^{-} \rightarrow \nu_{\tau} V^{-}\right)}{B\left(\tau^{-} \rightarrow \nu_{\tau} e^{-} \bar{\nu}_{e}\right)} \frac{1}{N_{V}} \frac{d N_{V}}{d s}\left[\left(1-\frac{s}{m_{\tau}^{2}}\right)^{2}\left(1+\frac{2 s}{m_{\tau}^{2}}\right)\right]^{-1}
$$

where the normalized invariant mass-squared distribution $\left(1 / N_{V}\right) d N_{V} / d s$ for a given hadronic mass $\sqrt{s}$ is divided by the kinematic factor on the right [22] and the branching ratio of $\tau$ decays to hadronic state $V$ is normalized to the branching ratio of the electron channel [22].

Since isospin is not an exact symmetry, the data must be corrected for electromagnetic effects and mass differences between the up and down quarks. $S_{E W}$ accounts for electroweak radiative corrections [19], $\left|V_{u d}\right|^{2}=0.9748 \pm 0.0010$ denotes the CKM weak mixing matrix element [19]. Some of these corrections are also fairly large, at the level of a few percent. Therefore, as with the $e^{+} e^{-}$data, the determination of the corrections are very important when calculating $a_{\mu}^{\mathrm{Had}}$.

The value of $a_{\mu}^{\mathrm{Had}}(1)$ has been determined by Davier, et. al. $[19,23]$ using both $e^{+} e^{-}$ and $\tau$ data by tabulating and subsequently integrating all data currently available. Fig. 1.6 shows the hadronic cross section as a function of center-of-mass energy squared, up to approximately $2.2 \mathrm{GeV}^{2}$, where both the $e^{+} e^{-}$and $\tau$ data are plotted on a log scale. The importance of the energy region below $s<1.8 \mathrm{GeV}$ is evident from this plot, which shows the $\rho$ resonance near $(770 \mathrm{MeV})^{2}$.

Although the $e^{+} e^{-}$and $\tau$ decay data in Fig. 1.6 appear to agree well, the calculated values of $a_{\mu}^{\mathrm{Had}}(1)$ actually differ by about $1.8 \sigma$ :

$$
a_{\mu}^{\mathrm{Had}}\left(1, e^{+} e^{-}-\text {based }\right)=696.3 \pm 6.2 \pm 3.6 \times 10^{-10}
$$




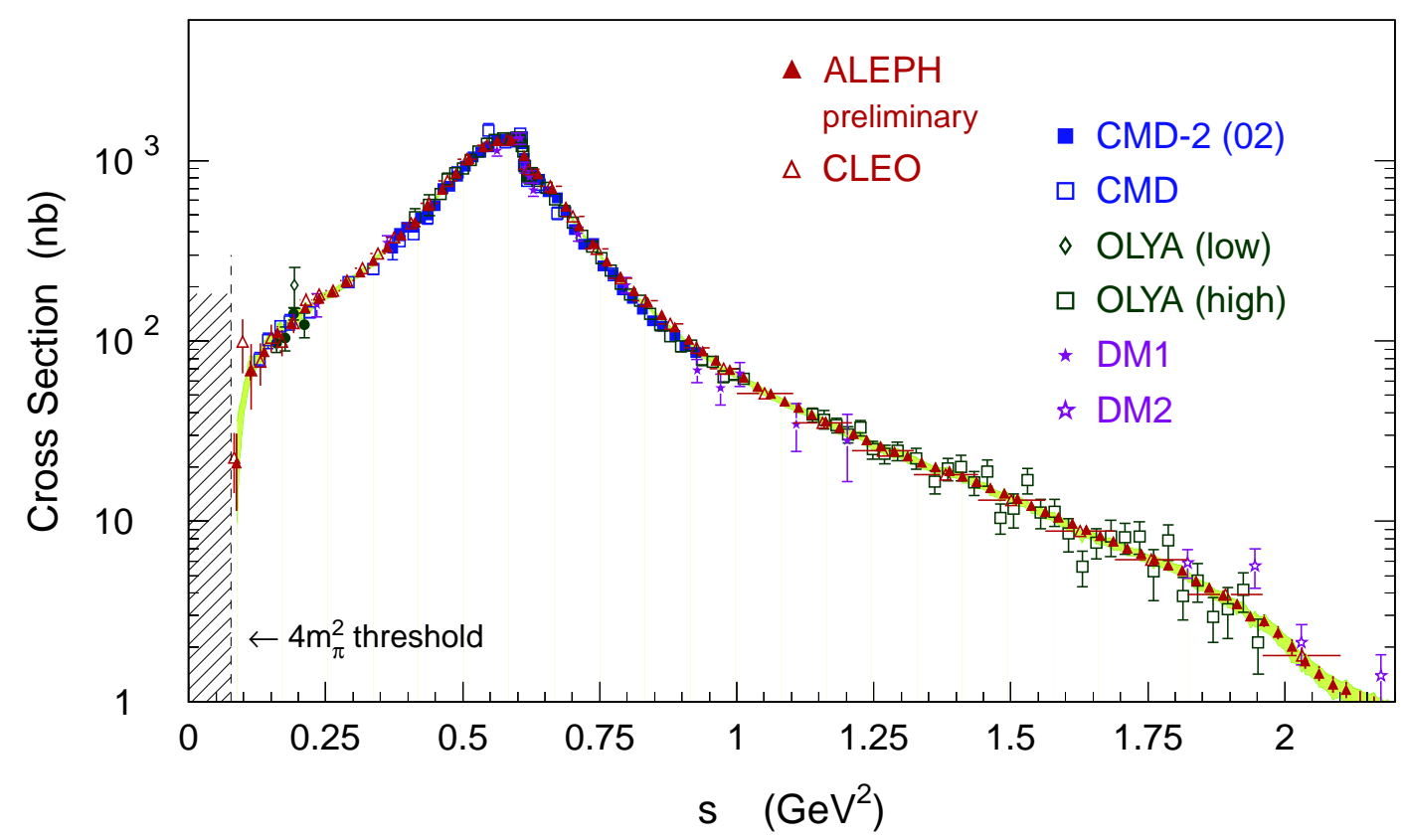

Figure 1.6: $\sigma\left(e^{+} e^{-} \rightarrow\right.$ hadrons $)$ vs. energy squared, $s$, as determined by various experiments over the last 20 years. Figure provided by Davier, et. al.[19].

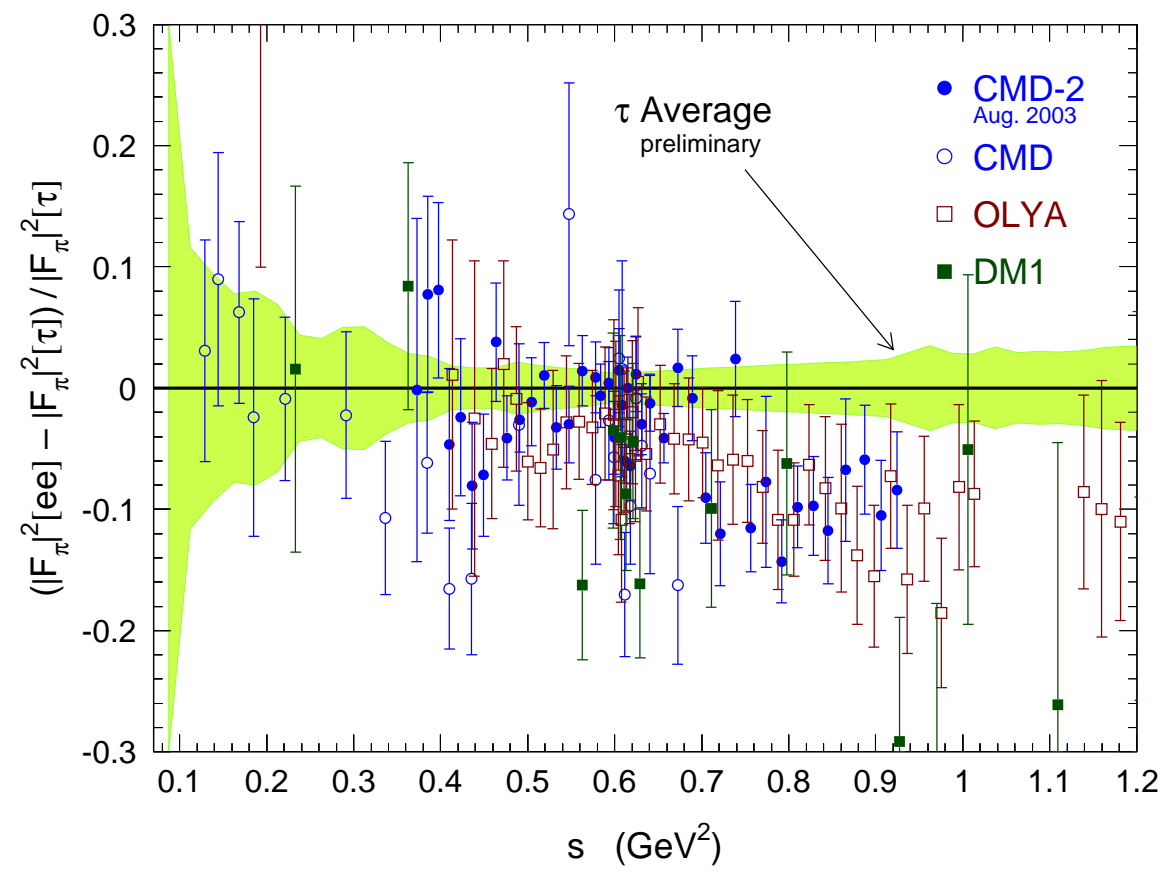

Figure 1.7: Difference between $e^{+} e^{-}$and $\tau$ decay spectral functions. Figure provided by Davier, et. al.[23]. 
and

$$
a_{\mu}^{\text {Had }}(1, \tau \text {-based })=711.0 \pm 5.0 \pm 0.8 \pm 2.8 \times 10^{-10}
$$

where the first uncertainty is experimental, the second uncertainty is from radiative corrections, and in the case of the $\tau$-based analysis, the third uncertainty arises from the applied isospin breaking corrections. Since the difference between the $e^{+} e^{-}$and $\tau$-based calculations of $a_{\mu}$ is $\sim 1.2 \mathrm{ppm}$ of the total value of $a_{\mu}^{\mathrm{SM}}$, the interpretation of the experimental result relies heavily on which theoretical value of $a_{\mu}^{\mathrm{SM}}$ one chooses. For example, the difference between the experimental value of $a_{\mu}$ (based on the 2000 data analysis) is at the $1.9 \sigma$-level using the $e^{+} e^{-}$data, whereas it is only at the $0.7 \sigma$-level using the $\tau$ decay data.

Davier and colleagues have pointed out that the difference between the two results arises primarily from a difference at the level of $10 \%$ near the $\rho$ region[19, 23], as shown in Fig. 1.7. Ghozzi and Jegerlehner have pointed out that mass and width differences between the $\rho^{0}$ and $\rho^{ \pm}$could very well be the cause of the difference between $e^{+} e^{-}$and $\tau$ decay[24]. However, Davier argues that even assuming a larger mass difference of $2.3 \pm 0.8 \mathrm{MeV}$, which seem to be favored by the data, there is still a discrepancy in the normalization of the spectral functions of $3.3 \%[25,26]$. In fact, this even makes the discrepancy between the $e^{+} e^{-}$and $\tau$ worse, raising the value of $a_{\mu}^{\mathrm{Had}}\left(1, \tau\right.$-based) by $5.4 \times 10^{-10}$. Obviously, until this issue is resolved, interpretation of the experimental value $a_{\mu}^{\exp }$ remains problematic.

\subsubsection{Higher order hadronic contributions}

The higher order hadronic vacuum polarization terms may also be related to the experimental data used in calculating the first order hadronic term. The most recent calculations were made by Krause [27] and Alemany, Davier and Hocker [20], in which they found

$$
a_{\mu}^{\text {Had }}(\text { h.o. })=-10.1(0.6) \times 10^{-10}
$$

However, there is another kind of hadronic contribution involving four photons coupled to an intermediate hadronic state, shown in Fig. 1.8(a), which cannot be directly related to any experimental data. These processes are referred to as hadronic light-by-light (HLBL) scattering. HLBL is dominated by the one-particle reducible pion-exchange pieces of the rank-four hadronic vacuum polarization tensor $\Pi_{\mu \nu \lambda \rho}\left(q_{1}, q_{2}, q_{3}\right)$ [28]. The pionic exchange, illustrated in Fig. 1.8(b), involves the double off-shell pion-photon-photon transition form factor $F_{\pi^{0} \gamma^{*} \gamma^{*}}\left(q_{1}^{2}, q_{2}^{2}\right)$. Since this quantity cannot be described by current data, we must rely on low-energy hadronic models to calculate the HLBL contribution to $a_{\mu}$.

Since the first calculation of HLBL in 1986 by Kinoshita, et. al. [29], the sign of the contribution has changed twice, from positive to negative and back to positive. Remarkably, the change from positive to negative was confirmed by three independent analyses [30, 31, 32] (one of which was a change after finding a mistake in [29]), and $a_{\mu}^{\mathrm{Had}}$ (HLBL) was found to be $\sim-8(2) \times 10^{-10}$.

However, spurred by the measurement of $a_{\mu}$ from data collected in 1999 and by the fact that previous calculations of the HLBL term employed low-energy QCD models that do not reproduce the correct short-distance QCD properties (but did predict reasonable values of $\left.a_{\mu}^{\text {Had }}(1)\right)$, Knecht and Nyeffeler recalculated the HLBL term using a large- $N_{c}$ model for

low-energy QCD, and found a value for $a_{\mu}^{\mathrm{Had}}(\mathrm{HLBL})$ that agreed in magnitude but had 


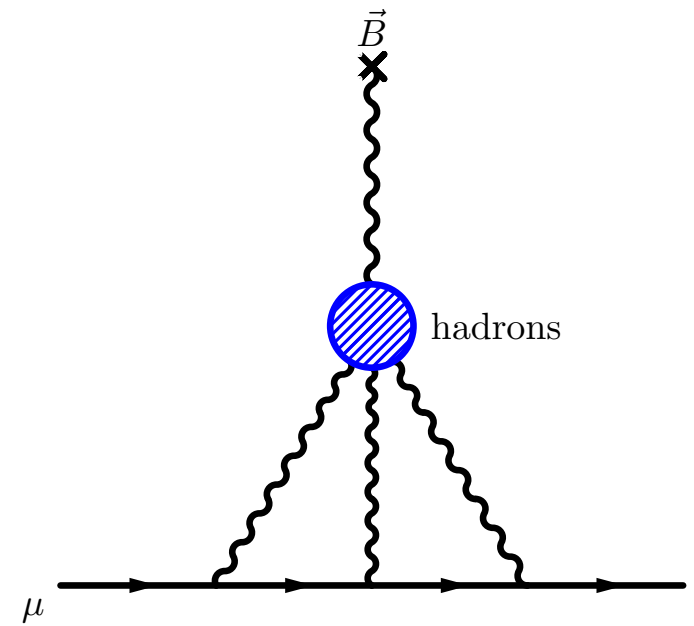

(a) The HLBL contribution consists of four photons coupled to an intermediate hadronic state.

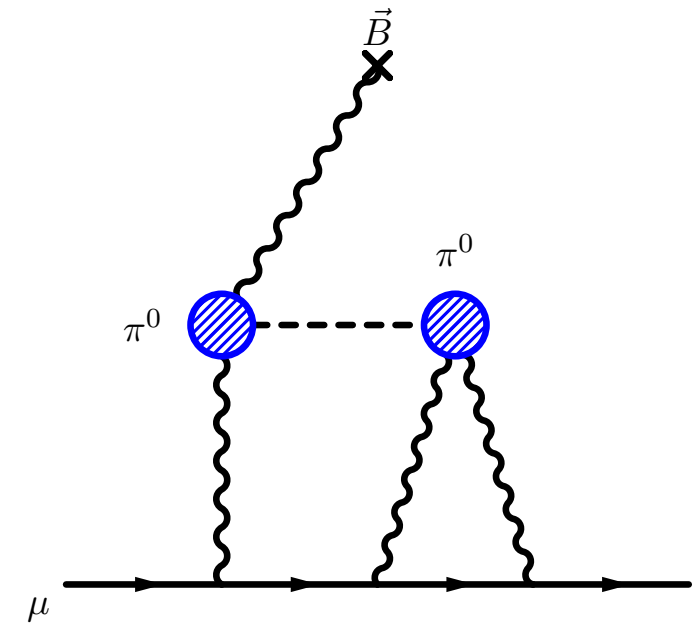

(b) The dominant pion-pole contribution to HLBL.

Figure 1.8: Hadronic light-by-light (HLBL) contribution.

a different sign. After much work by all parties, Hayakawa and Kinoshita indeed found a sign error $[33,34]$ in the treatment of the tensor $\epsilon_{\mu_{1} \mu_{2} \mu_{3} \mu_{4}}$ which affected primarily the pseudoscalar meson pole contribution to $a_{\mu}$. Neither the charged pion-loop contribution nor the quark-loop contributions were affected by the tensor. Similarly, Bijnens, et. al. found a sign mistake in the overall normalization factor of the numerically integrated pion pole contribution[35].

Although there is now general agreement ${ }^{3}$ on both the sign and magnitude of $a_{\mu}^{\mathrm{Had}}$ (HLBL) of $\sim 8 \times 10^{-10}$, there is some discrepancy among the uncertainties quoted by [28] $\left(4 \times 10^{-10}\right)$, [33] $\left(1.54 \times 10^{-10}\right)$ and [35] $\left(3.2 \times 10^{-10}\right)$. We therefore use the conservative larger uncertainty, and $a_{\mu}^{\mathrm{Had}}(\mathrm{HLBL})=8(4) \times 10^{-10}$. Obviously, since all calculations of $a_{\mu}^{\mathrm{Had}}(\mathrm{HLBL})$ are model dependent, this contribution to the muon anomalous magnetic moment poses the largest problem for any theoretical calculation of $a_{\mu}^{S M}$. Whereas the uncertainty on $a_{\mu}^{\operatorname{Had}}(1)$ will eventually be pushed below the level of uncertainty on $a_{\mu}^{\text {Had }}$ (HLBL) as more and better data are obtained, the uncertainty on $a_{\mu}^{\mathrm{Had}}(\mathrm{HLBL})$ is unlikely to be reduced in the near future, barring a breakthrough in lattice or other QCD calculations.

Adding all hadronic contributions together, we find

$$
a_{\mu}^{\text {Had }}\left(e^{+} e^{-} \text {-based }\right)=694.2(8.3) \times 10^{-10}
$$

and

$$
a_{\mu}^{\text {Had }}(\tau \text {-based })=708.9(7.1) \times 10^{-10}
$$

and adding all known contributions to $a_{\mu}^{\mathrm{SM}}\left(a_{\mu}^{\mathrm{QED}}, a_{\mu}^{\mathrm{Weak}}\right.$ and $\left.a_{\mu}^{\mathrm{Had}}\right)$ together we find (adding

\footnotetext{
${ }^{3}$ An exception is Melnikov and Vainshtein [36] who argue for a $\sim 50 \%$ increase in the central value of the HLBL term due to constraints on the $\pi^{0}$-pole contribution which were ignored by previous analyses $[28,33,35]$.
} 
the errors in quadruature)

$$
a_{\mu}^{\mathrm{SM}}\left(e^{+} e^{-} \text {-based }\right)=11659179.7(8.3) \times 10^{-10}
$$

and

$$
a_{\mu}^{\mathrm{SM}}(\tau \text {-based })=11659194.4(7.1) \times 10^{-10}
$$

\subsection{Sensitivity of $a_{\mu}$ to Physics Beyond the Standard Model}

In 1956, it was pointed out by Berestetskii and colleagues [37] that an accurate measurement of $a_{\mu}$ would test QED at much higher $q^{2}$ than the current measurements of $a_{e}$ provided at the time. In 1957 Schwinger suggested that a measurement of $a_{\mu}$ would test for the existence of new fields that couple to the muon which could have explained the $\mu-e$ mass difference [38]. Therefore, even before the Standard Model of particle physics was developed, it was recognized that precision measurements of $a_{\mu}$ could provide much insight into as-yet undiscovered physics.

The development and consequent precision tests of the Standard Model since the 1970's is one of the greatest accomplishments in the field of particle physics. However, we know that the model is incomplete, for it fails to explain some very fundamental questions, such as the origin of mass, the hierarchy problem ${ }^{4}$, baryogenesis, and the role of gravity, just to name a few. For quite some time now, extensions to the Standard Model have been sought to resolve several of these fundamental issues, many of which have direct implications on the value of $a_{\mu}$. Examples of such extensions are supersymmetry (SUSY), muon substructure and extra dimensions. A difference between $a_{\mu}^{\exp }$ and $a_{\mu}^{\mathrm{SM}}$ therefore constrains the parameters of these theoretical extension to the Standard Model.

In general, the effects of any new physics on $a_{\mu}$ are proportional to $\left(m_{\mu} / \Lambda\right)^{2}$, where $\Lambda$ is the energy scale of the new physics. For example, the current measurements of $a_{\mu}$ may be particularly sensitive to SUSY. Assuming large value of $\tan \beta$ (in the range 4-40) and assuming similar masses for the two lightest supersymmetric partners (the chargino and the sneutrino), Czarnecki and Marciano find[8]

$$
a_{\mu}^{\mathrm{SUSY}} \simeq 130 \times 10^{-11}\left(\frac{100 \mathrm{GeV}}{M}\right)^{2} \tan \beta
$$

where $M$ is the mass scale of the SUSY particles ${ }^{5}$. Therefore, in this scenario, SUSY masses in the range of hundreds of $\mathrm{GeV}$ to about $1 \mathrm{TeV}$ would contribute at a discernible level in the current measurements of $a_{\mu}$, which are sensitive at the $10^{-10}$ level.

\footnotetext{
${ }^{4}$ The hierarchy problem of the Standard Model refers to the unnatural size $\left(10^{19}\right.$ orders of magnitude) separation between the electroweak symmetry breaking scale and the Plank scale.

${ }^{5} \tan \beta$ is the ratio of the vacuum expectation values (VEV) of the two Higgs doublets in the Minimal Supersymmetric Model.
} 


\section{Chapter 2}

\section{Experiment E821 at BNL}

\subsection{Previous Measurements of $a_{\mu}$}

All previous and current measurements of the anomalous magnetic moment of the muon are based on two basic principles: muons born from pion decay in flight are polarized, and muons themselves decay anisotropically. In 1958, a group at the European Organization for Nuclear Research (CERN) began studying ways in which to make a measurement of $a_{\mu}$. Over the next 20 years, three very successful experiments at CERN measured $a_{\mu}$; each experiment improved on the previous one, and each experiment probed new scales of physics.

The basic principle of each CERN experiment (and that of the current BNL experiment) is to trap polarized muons in a magnetic field and measure the evolution of $\mathbf{s} \cdot \mathbf{p}$ as a function of time. As will be described more fully in Section 2.2, the time dependence of the angle $\theta$ between $\mathbf{s}$ and $\mathbf{p}$ of a muon orbiting in a magnetic field is

$$
\omega_{a}=\frac{d \theta}{d t}=a_{\mu} \frac{e B}{m}
$$

Therefore, simultaneous measurements of both $\omega_{a}$ and $B$ determine $a_{\mu}$.

The very first measurement of the anomalous magnetic moment of the muon was made at CERN in 1961, where $a_{\mu}$ was measured to be 0.001162(5) [39]. Muons from the CERN Synchro-cyclotron were injected into almost circular orbits in a 6 meter long magnet with a magnetic field of $1.58 \mathrm{~T}$. A carefully shaped gradient in the magnetic field made the circular orbits drift along the horizontal axis of the magnet. The spin direction of the exiting muons was measured by stopping the muons in a field-free absorber and recording the decay electrons in forward and backward counter telescopes [40]. This first result confirmed the QED predictions for a point particle.

The measurement in the first experiment was severely limited by the muon lifetime of $2.2 \mu \mathrm{s}$, which allowed the precession to be measured over only two cycles, or $\sim 8 \mu \mathrm{s}$. The next experiment therefore sought to decrease the experimental error by increasing the number of precession cycles using relativistic muons in a weak-magnetic-focusing ring magnet $\left(B_{0}=1.72 \mathrm{~T}, n=0.13\right)[40]$. Stored muons were obtained by first injecting a focused proton beam onto a target inside the storage ring; pions of momentum $\sim 1.3$ 
$\mathrm{GeV} / \mathrm{c}$ created in the collisions begin to turn in the storage ring, and a small fraction of muons from pion decay, born at small forward angles and on average polarized, fall onto stable orbits. The second CERN experiment found $a_{\mu}$ to be $116616 \pm 31 \times 10^{-8}(270 \mathrm{ppm})$, reducing the uncertainty on the experimental value by a factor of 16 . However, the use of magnetic field gradients to focus the muons destroyed the homogeneity of the magnetic field and introduced a limiting systematic error on the measurement of the average field seen by the muons.

A third and final set of measurements were made using an approach similar to that of the second experiment, although with a radically different storage ring. Most of the improvements over the second CERN experiment arose from increasing the relativistic factor, $\gamma$, from 12 to 29.3. Not only did this increase the number of $(g-2)$ oscillations per muon lifetime, but, more importantly, at this special $\gamma$ the effect of electric fields on the spin precession frequency vanishes. In the CERN III experiment, electrostatic quadrupoles were used to vertically focus the beam in the storage ring, eliminating the need for gradients in the magnetic field and allowing for a more precise determination of the magnetic field. Finally, by directly injecting pions, the background was decreased which allowed an increase in beam intensity. The CERN III experiment was able to decrease the statistical and systematic error of $a_{\mu}$ by a factor of nearly 37, and confirmed the theoretical predictions of the first-order hadronic loop contributions to $a_{\mu}$ :

$$
\begin{aligned}
& a_{\mu^{+}}=1165911(11) \times 10^{-9}(10 p p m) \\
& a_{\mu^{-}}=1165937(12) \times 10^{-9}(11 p p m) \\
& a_{\mu}=1165924(8.5) \times 10^{-9}(7.3 p p m)
\end{aligned}
$$

where $a_{\mu}$ is the average of the two $\mu^{+}$and $\mu^{-}$values.

\subsection{Principle of the Experiment}

The measurement of the anomalous magnetic moment of the muon at BNL, designated "E821", is based on the same methods as the third measurement at CERN in 1979, with the goal of a 20 -fold improvement in the error. The improvement in the statistical error is made feasible by the $\sim 200 \times$ increased muon flux produced by the BNL AGS, improved energy resolution and the muon injection scheme described in Section 2.4.2.

Polarized muons are injected into a storage ring with a vertical magnetic field $\vec{B}=$ $\left(0, B_{y}, 0\right)$. Electrostatic quadrupoles, which produce electric fields in the radial and vertical directions, $\vec{E}=\left(E_{x}(\vec{r}), E_{y}(\vec{r}), 0\right)$, vertically focus the muon beam. The equations of motion for the spin vector of a muon in flight in external magnetic and electric fields were first calculated by Thomas [41], but often referred to as the BMT equations (for Bargmann, Michel and Telegdi's work [42]):

$$
\vec{\omega}_{s}=\frac{e}{m}\left[\left(\frac{g}{2}-1+\frac{1}{\gamma}\right) \vec{B}-\left(\frac{g}{2}-1\right) \frac{\gamma}{\gamma+1}(\vec{\beta} \cdot \vec{B}) \vec{\beta}-\left(\frac{g}{2}-\frac{\gamma}{\gamma+1}\right)(\vec{\beta} \times \vec{E})\right]
$$

where $e$ is the charge of the muon, and $m$ is the mass of the muon.

The equations of motion for such a charged particle moving in external magnetic and 


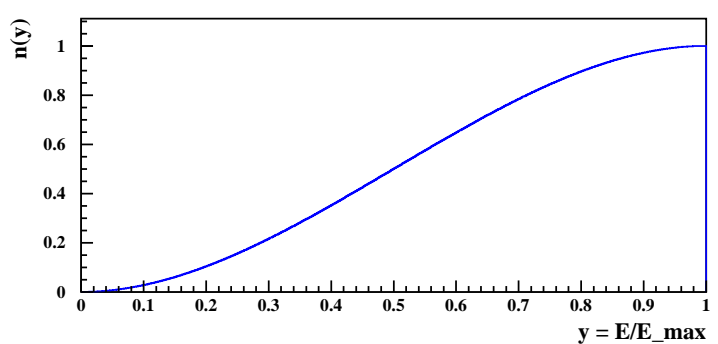

(a) $n(y)$

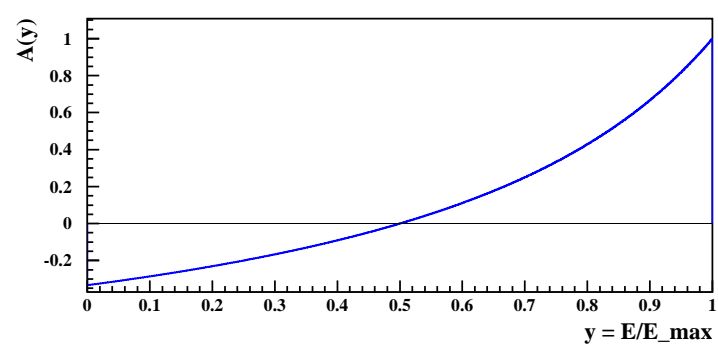

(b) $A(y)$

Figure 2.1: $n(y)$ and $A(y)$, the energy-dependent weighting function and asymmetry of muon decay in the rest frame of the muon.

electric fields are

$$
\dot{\vec{p}}=\overrightarrow{\omega_{c}} \times \vec{p}=e(\vec{\beta} \times \vec{B}+\vec{E})
$$

where the cyclotron frequency $\vec{\omega}_{c}$ is:

$$
\vec{\omega}_{c}=\frac{e}{m}\left[\frac{1}{\gamma} \vec{B}-\frac{\gamma}{\gamma^{2}-1}(\vec{\beta} \times \vec{E})\right]
$$

The difference between these two frequencies, $\vec{\omega}_{s}-\vec{\omega}_{c}=\vec{\omega}_{a}$ is known as the "spin difference frequency". Grouping similar terms and using $a_{\mu}=(g-2) / 2$ we find

$$
\vec{\omega}_{a}=\frac{e}{m}\left[a_{\mu} \vec{B}-a_{\mu}\left(\frac{\gamma}{\gamma+1}\right)(\vec{\beta} \cdot \vec{B}) \vec{\beta}-\left(a_{\mu}-\frac{1}{\gamma^{2}-1}\right)(\vec{\beta} \times \vec{E})\right]
$$

Assuming the motion of the muons is always perpendicular to the magnetic field of the storage ring, we may ignore the $\vec{\beta} \cdot \vec{B}$ terms. Furthermore, we choose the $\gamma$ of the muons to be the "magic" value $\simeq 29.3$, so that the $\vec{\beta} \times \vec{E}$ term drops out. The equation for the spin precession frequency therefore reduces to

$$
\vec{\omega}_{a}=\frac{e}{m} a_{\mu} \vec{B}
$$

and the measurement of $a_{\mu}$ requires two independent measurements, one of $\omega_{a}$ and one of $B$.

Two assumptions were made in order to arrive at Eq. 2.9: $\vec{\beta} \cdot \vec{B}=0$ and $a_{\mu}-1 /\left(\gamma^{2}-1\right)=$ 0 . Neither of these assumptions are exactly true for all muons, and small corrections are made to the measured spin precession frequency to account for these assumptions. The correction for the fact that the muon's trajectory is not always perpendicular to the magnetic field is called the pitch correction, and the correction for the fact that the injected muons are momentum dispersed, and thus not all muons have the magic momentum, is called the radial E-field correction. Both these corrections are of the order $\sim 0.3-0.4 \mathrm{ppm}$ and will be discussed later.

The measurement of the spin precession frequency relies on the parity-violating nature 
of the muon weak decay, $\mu^{-} \rightarrow e^{-} \nu_{e} \bar{\nu}_{\mu}$. In the rest frame of the muon, the differential probability that the muon will decay into an electron with normalized energy $y=E / E_{\max }$, where $E_{\max }=52.8 \mathrm{MeV}$, and angle $\theta_{s}$ relative to the muon spin is [43]

$$
\frac{d P}{d y d \Omega}=n(y)\left[1+A(y) \cos \theta_{s}\right]
$$

where

$$
n(y)=y^{2}(3-2 y)
$$

and

$$
A(y)=\frac{2 y-1}{3-2 y}
$$

The functions $n(y)$ and $A(y)$ are plotted in Fig. 2.1, from which we see immediately that many more muons decay to electrons with $y>0.5$ than with $y<0.5$ and the average asymmetry of the decay electrons with $y>0.5$ is positive, whereas it is negative for those electrons with $y<0.5$. Therefore we find that electrons are emitted preferentially in the direction of the muon's spin. Indeed, simply integrating over all energies, we see that

$$
\frac{d P}{d \Omega}=\frac{1}{2}\left(1+\frac{\cos \theta_{s}}{3}\right)
$$

In the lab frame, the energy of the decay electron is

$$
\begin{aligned}
E_{\mathrm{lab}} & =\gamma\left(E+\beta p \cos \theta^{*}\right) \\
& \simeq \gamma E\left(1+\cos \theta^{*}\right)
\end{aligned}
$$

where $E$ and $p$ are the energy and momentum of the electron in the muon rest frame, $\gamma$ comes from the relativistic boost and $\theta^{*}$ is the decay angle in the laboratory frame. High-energy electrons therefore are emitted along the direction of the of the spin of the muon.

Taking into account that in the laboratory frame the muon's spin precesses relative to the momentum vector (therefore, $\cos \theta_{s} \rightarrow \cos \left(\omega_{a} t+\phi\right)$ ) and that the muon decays with a lifetime of $\gamma \tau_{0}=\tau$, one expects the number of observed decay electrons above an applied energy threshold $E_{t h}$ to be

$$
N(t)=N_{0}\left(E_{t h}\right) e^{-t / \tau}\left[1+A\left(E_{t h}\right) \cos \left(\omega_{a} t+\phi\right)\right]
$$

The phase $\phi$ comes from the initial spin polarization of the muons. By plotting the number of decay electrons observed as a function of time, one may extract $\omega_{a}$ by fitting the data to the above equation.

Since the determination of $\omega_{a}$ is based on the number of counts, there is a statistical uncertainty on $\omega_{a}$. If $\phi$ is not determined a priori, then one can easily show that the statistical error on $\omega_{a}$ is approximately

$$
\frac{\delta \omega_{a}}{\omega_{a}} \simeq \frac{\sqrt{2}}{\omega_{a} \gamma \tau_{0} A \sqrt{N}}
$$

The factor of $\sqrt{2}$ comes from the strong correlation between the phase $\phi$ and the frequency 
$\omega_{a}$. Since both $A$ and $N$ depend on energy-threshold and since we wish to minimize the statistical uncertainty on $\omega_{a}$, the energy-threshold is chosen such that the product $N A^{2}$ is maximized.

The magnetic field is determined with Nuclear Magnetic Resonance (NMR), the measurement of the Larmor spin precession frequency of proton in water

$$
\omega_{L}(p)=\omega_{p}=g_{p}\left(\frac{e B}{2 m_{p}}\right)
$$

where $g_{p}$ is the gyromagnetic ratio of the proton. Solving for B, we have

$$
a_{\mu}=\frac{\omega_{a}}{\omega_{p}} \frac{m_{\mu}}{m_{p}} \frac{g_{p}}{2}=\frac{\omega_{a}}{\omega_{p}} \frac{m_{\mu}}{m_{p}} \frac{g_{p}}{g_{\mu}}\left(1+a_{\mu}\right)
$$

We define

$$
\lambda=\frac{m_{p}}{m_{\mu}} \frac{g_{\mu}}{g_{p}}=\frac{\mu_{\mu}}{\mu_{p}}
$$

and

$$
\mathcal{R}=\frac{\omega_{a}}{\omega_{p}}
$$

and therefore

$$
a_{\mu}=\frac{\mathcal{R}}{\lambda-\mathcal{R}}
$$

The value of $\lambda$ used in this experiment comes from the Particle Data Group's analysis [44], which combines experimental and theoretical values of the hyperfine structure in muonium interval $\Delta \nu$ to obtain $\lambda$ with an uncertainty of $0.03 \mathrm{ppm}$. $\lambda$ has also been determined in another experiment that measured the Zeeman transition frequency in muonium [45] to within $0.12 \mathrm{ppm}$. The Zeeman transition frequency measurement is in excellent agreement with the muonium hyperfine experiment. In fact, the same NMR probe used in this experiment for absolute calibration of $\omega_{p}$ was also used in the muonium Zeeman effect experiment, so that any systematic uncertainties involved with the value of $\lambda$ are divided out in the measurement of $a_{\mu}$.

\subsection{Production and Injection of Polarized Muons}

\subsubsection{AGS Beamline}

Polarized muons are produced at BNL via the AGS and relies on the parity-violating decay of the pion, $\pi^{-} \rightarrow \mu^{-} \nu_{\mu}$. Protons are initially accelerated to $200 \mathrm{MeV} / \mathrm{c}$ by a linear accelerator (LINAC), then raised to $1.6 \mathrm{GeV} / \mathrm{c}$ by a BOOSTER after which they are transferred to the AGS where they are accelerated to a momentum of $24 \mathrm{GeV} / \mathrm{c}$. The protons are then extracted from the AGS, hit a target and produce, amongst other debris-particles, large numbers of low energy pions. The pions are then momentum-selected and transported along a 72 meter-long straight channel in which approximately one third of the pions decay to muons. At the end of the straight channel, the particles are again momentum-selected and injected into the $g$-2 storage ring. The AGS/g-2 beamline is shown in Fig. 2.2. The 


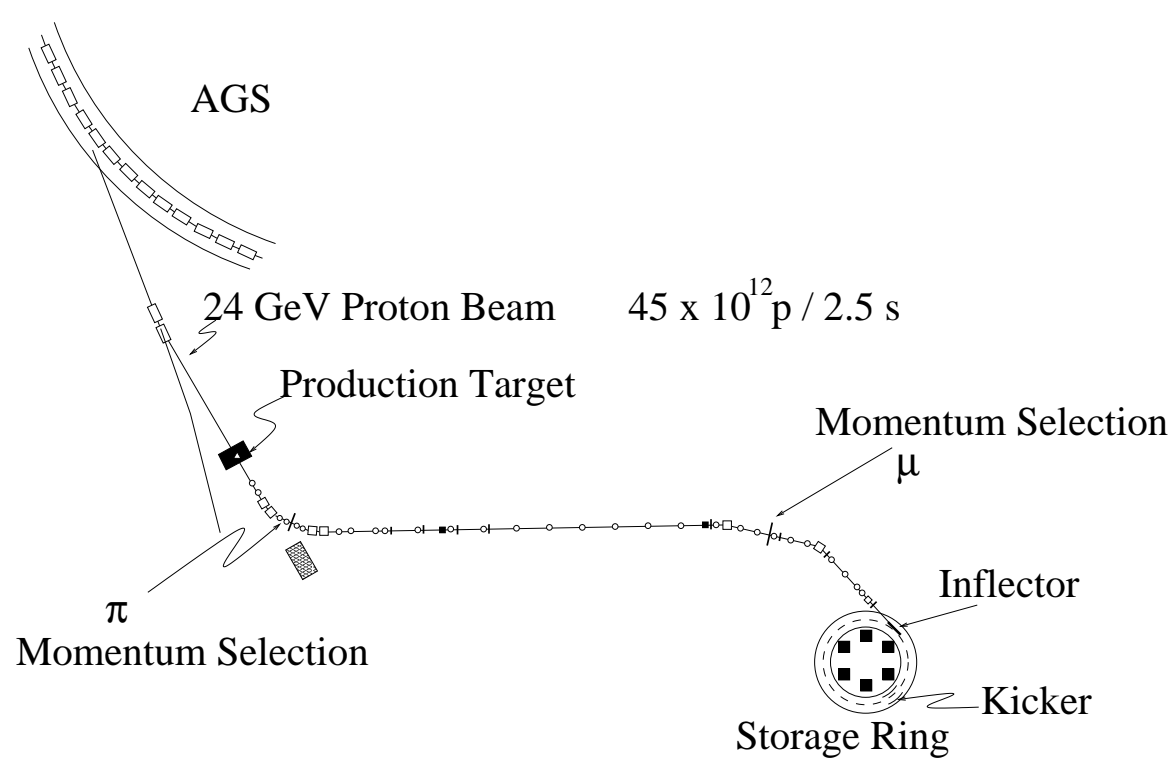

Figure 2.2: Top view of the AGS beamline. Protons are extracted from the AGS and strike a Ni target. At the first bend the pions are momentum-selected for $p_{\pi}=1.017 p_{\text {magic }}$. The second bend in the beamline momentum-selects muons at $p_{\mu}=p_{\text {magic }}$, after which the muons enter the $g$-2 storage ring through a superconducting inflector.

AGS cycle is approximately 2.5 seconds, and in 2001 the protons were extracted at the end of the AGS cycle in 12 bunches of RMS widths $\sim 22 \mathrm{~ns}$, each bunch separated by $\sim 33 \mathrm{~ms}$. The AGS cyclotron frequency is $2.694 \mu \mathrm{s}$.

The proton bunches strike a water-cooled nickel production target designed specifically to handle the high flux needed for this experiment. The target is made of 24 nickel disks, each separated by $1.6 \mathrm{~mm}$. The disks themselves are $15 \mathrm{~cm}$ in diameter and $6.4 \mathrm{~mm}$ thick. The disks rotate around an axis parallel to the proton beam through a water bath and are struck by the proton beam near their outer edge $(\sim 7.5 \mathrm{~cm}$ from the center $)$. The nickel target was designed to handle thermal stresses induced by a maximum beam flux of about 7.5 teraprotons (TP) on target per bunch. In 2001, the maximum AGS beam intensity obtained was $\sim 56 \mathrm{TP}$ per AGS cycle, or $\sim 4.6 \mathrm{TP}$ per bunch.

Many kinds of particles are produced in the proton-nickel collision, including pions $\left(\pi^{ \pm}, \pi^{0}\right)$, electrons $\left(e^{ \pm}\right)$, and protons $(p$ and $\bar{p})$. In order to maximize the number of stored muons in the $g-2$ storage ring while reducing the number of other particles that can produce a large background flash in the $g$-2 electron calorimeters, the particles exiting the target are charge and momentum-selected at $3.147 \mathrm{GeV} / \mathrm{c}(1.7 \%$ above the magic momentum $\left.p_{0}=3.094 \mathrm{GeV} / \mathrm{c}\right)$ at the first bend in the beamline.

Since the pion has spin 0 and neutrinos are left-handed, conservation of angular momentum in the rest frame of the pion requires the spin of the decay muon to have the same helicity as the associated neutrino. Therefore selection of forward-going decay muons results in a polarization of $\sim 95 \%$ in the longitudinal direction. In 2001 , the second bend of the beamline in Fig. 2.2 selected negative muons (which have positive helicity) of the magic 


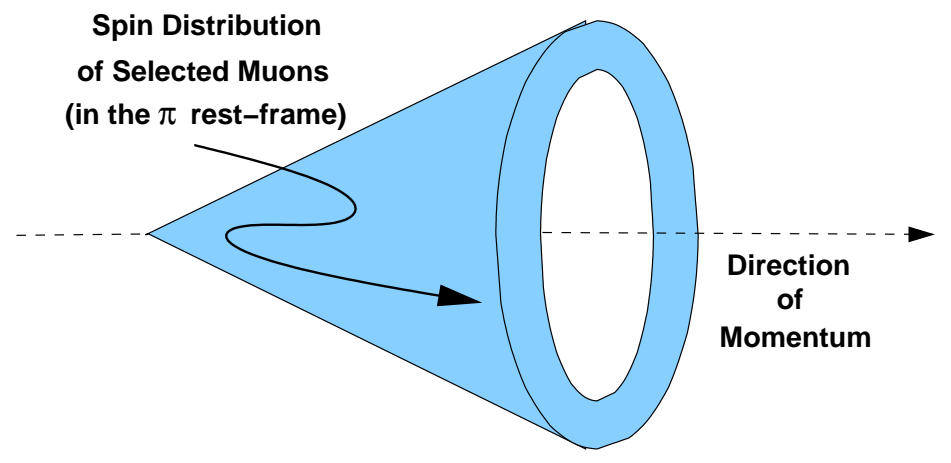

(a) Spin distribution of muons from pion decay.

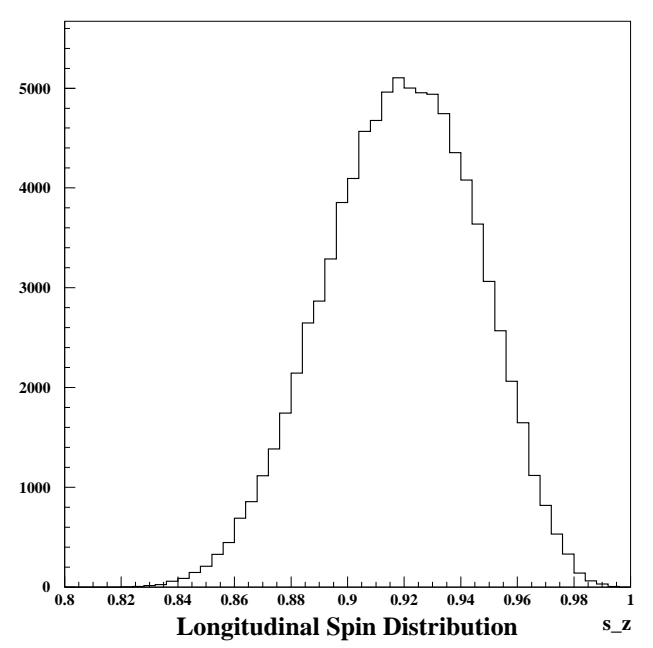

(b) Distribution of muon spins along the direction of motion of the beam.

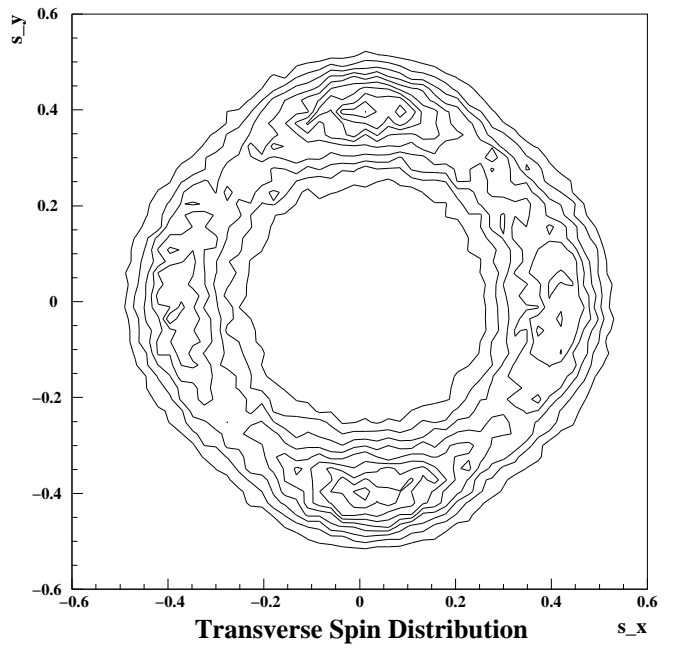

(c) Contour plot of the two-dimensional distribution of muon spins in the transverse plane of motion of the beam.

Figure 2.3: (a): Schematic of the spin distribution of muons obtained from pion decay due to momentum selection in the beamline. The muon spins are $\sim 93 \%$ polarized along the direction of momentum of the pions in lab . (b) and (c): Spin distributions as calculated by BTRAF, a beamline transport Monte Carlo simulation. 
momentum $(3.094 \mathrm{GeV} / \mathrm{c})$. The momentum-selection results in an annular-cone spin distribution as depicted in Fig. 2.3(a). The beamline was simulated with BTRAF[46], a Monte Carlo beamline transport simulation. Figs. 2.3(b) and 2.3(c) show the longitudinal spin and transverse spin distributions, respectively, obtained from BTRAF.

The pion/muon beam was initially tuned and then monitored on-line using a series of 10 Segmented Wire Ion Chambers (SWICs) positioned at various locations along the beamline. The SWICs consist of a cathode plane and 20 anode wires, positioned in both the radial and vertical directions, that sit inside a volume filled with a gas mixture of $50 \% \mathrm{Ar}$ and $50 \% \mathrm{CO}_{2}$ [47]. When a charged particle passes through the gas mixture, electron-ion pairs are produced and charge is collected at the anode wires, which are read out via a standalone CAMAC system [47]. The total amount of charge accumulated on each anode wire is proportional to the number of particles passing near that wire, allowing a determination of both radial and vertical positions of the beam.

\subsubsection{The Superconducting Inflector}

The muon beam enters into the storage ring via a hole in the back leg of the ring magnet, as shown in Fig. 2.4. The beam is injected at an angle of $\sim 11 \mathrm{mrad}$ with respect to the tangent of the central design orbit of the storage ring, and passes through both the storage ring's fringe and main fields before entering the storage ring. In order to protect the injected beam from the fringing magnetic field lines upstream and to push the beam in the proper direction, a device is required that provides an integral field of $\sim 2.6 \mathrm{~T} \cdot \mathrm{m}$ near the entrance of the ring and maintains the homogeneity of the main magnetic field of the storage ring.

Various options were considered, but in the end a direct-current non-ferrous superconducting septum magnet, or inflector, was constructed [48]. The choice of a DC superconducting inflector instead of the more typical pulsed magnetic inflector was necessitated by the fact that a pulsed magnet that could both handle the heating-stress and produce a small-enough fringe field would have been extremely difficult to construct.

Fig. 2.5 shows a schematic cross-section views of the inflector. The inflector itself is 1.7 $\mathrm{m}$ long, and employs Aluminum-stabilized superconductor to make the coils and cryostat design compact and to minimize the interactions of the incoming muon/pion beam. A 1.5 $\mathrm{T}$ field is produced close to the storage region by 88 turns of the superconducting coil ( 52 for the outer, 36 for the inner coil) using a truncated double cosine theta design. This design maximizes the useful field region, yet minimizes the total ampere-turns [48]. A superconducting magnetic flux shield surrounds the coils in order to trap the multipole fringe field of the inflector inside itself so that the measurement of the $g-2$ storage ring field is not compromised.

In order to have the storage ring magnetic field be as uniform as possible, there are no gaps in the yoke or pole pieces (see Section 2.4.1), and the inflector must necessarily sit outside the storage ring. In addition, because of engineering constraints, the aperture of the inflector could not be made as large as that of the storage ring, resulting in a phase-space mismatch for muon injection. The consequence of this will be discussed in Section 2.4.2. 


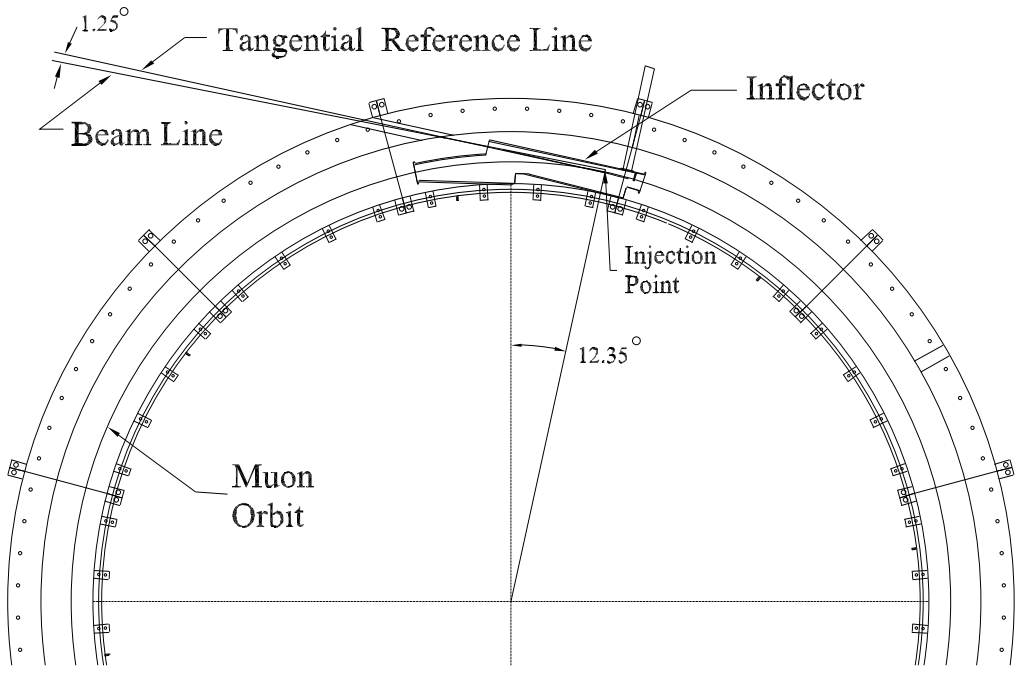

Figure 2.4: Top view of the injection point of the beam into the $g$ - 2 storage ring. An inflector is necessary to cancel the local fringe field of the storage ring while at the same time moving the beam onto a properly aligned trajectory.

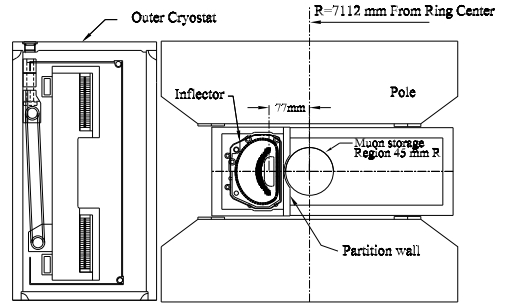

(a) Cross-section schematic of the storage ring at the point of injection. The inflector necessarily sits outside of the central storage region.

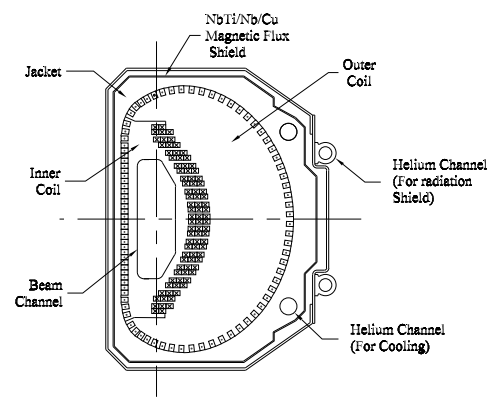

(b) Close-up cross-section schematic of the inflector.

Figure 2.5: Cross-section view of the $g-2$ superconducting inflector, which connects the AGS beamline to the $g-2$ storage ring by canceling the ring fringe field and producing a magnetic field matching that inside the ring's storage aperture. Note, however, that there is no matching of phase space parameters between the inflector and storage ring. 


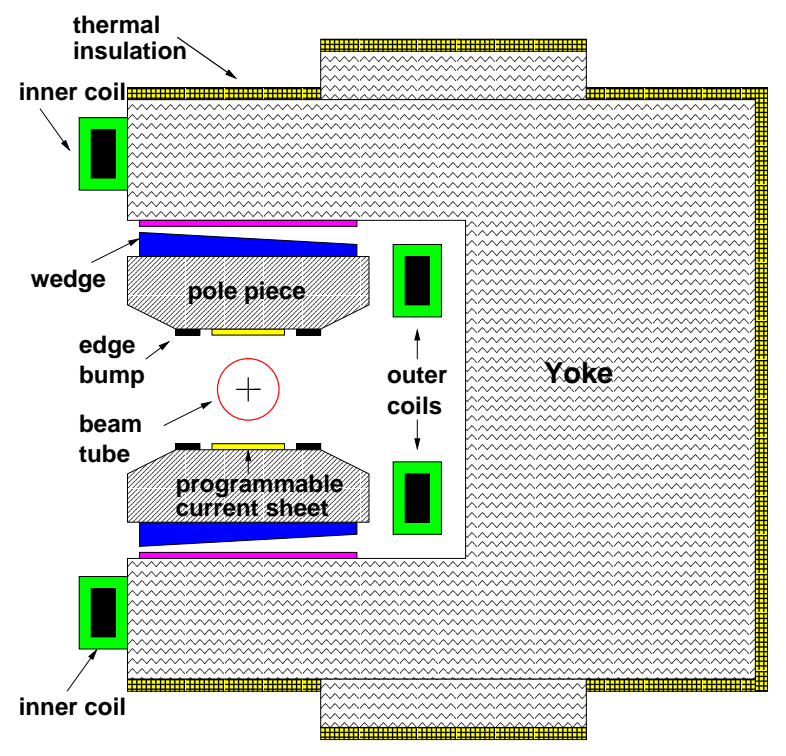

Figure 2.6: Cross section view of the $g-2$ storage ring.

\subsection{Storage of Muons}

\subsubsection{The Muon g-2 Storage Ring}

The storage ring used in E821 is a continuous C-shaped, super-ferric (superconducting coils with an iron yoke) $1.451 \mathrm{~T}$ magnet of diameter $14.22 \mathrm{~m}$. A cross-sectional view of the magnet is shown in Fig. 2.6. The C-shape is dictated by the need to detect decay electrons on the inside of the storage ring. Superconducting coils, although much more expensive than copper coils, have several advantages which make a high-precision measurement of the magnetic field much more feasible: relative low power requirements, low ripple currents, thermal stability (once cold) and thermal independence of the coils and iron yoke.

Four $\mathrm{NbTi} / \mathrm{Cu}$ superconducting coils, each made of 24 turns, power the magnet: two at an outer radius drive the field across the storage ring gap, and two at inner radius above and below the mid-plane cancel the flux in the ring center and improve the field quality in the gap [49]. The coils are connected in series and operate at a current of $5200 \mathrm{~A}$ at a temperature of $4.5 \mathrm{~K}$; the current in the outer and inner coils flows in opposite directions. The temperature of the four coils is maintained using liquid helium in three ring cryostats; the outer coils share one and the two inner coils each have their own. The superconductor is operated well below its critical current of $10000 \mathrm{~A}$ at $4.5 \mathrm{~K}$ (which produces a $2 \mathrm{~T}$ field) [50].

In order to minimize the impact of inclusions and voids in the iron, the poles of the magnet are made of very pure continuous vacuum cast iron with a typical carbon content of $0.0003 \%$, whereas the yoke of the magnet is made of conventional quality magnet steel of typical carbon content of $0.07 \%$ [51]. The total weight of the magnet, about 750 metric tons, comes primarily from the yoke. Minimizing the cost of the steel required a minimal yoke cross-section, which in turn minimizes the total flux and requires a narrow pole. The 
narrow poles, however, make producing a very-uniform field over the $9 \mathrm{~cm}$ field aperture very difficult. Furthermore, although the effect of variations in the yoke reluctance is reduced by isolating the poles and yoke by wedge-shaped air gaps, this problem is never quite eliminated. Finally, because of its size, it was necessary to build the magnet from $3610^{\circ}$ pieces, all of which vary within reasonable tolerances. These tolerances, however, lead to large variations of the magnetic field when the pieces are assembled. A means to "shim" the magnet, so that large local field variations may be eliminated, is required.

\section{Shimming of the Magnet}

Fig. 2.6 shows three ways in which the magnetic field can be altered to produce a very homogeneous dipole magnetic field: edge shims, air-gap wedges and programmable current windings that sit on the pole pieces. The edge and wedge shims provide a means to passively alter the local magnetic field, whereas the programmable current sheets, or correcting surface coils, offer a means to actively adjust the magnetic field.

In addition to isolating the yoke and pole, the air gaps serve to shape the magnetic field in the storage aperture by the help of iron wedges: the slope of the wedge-shape is calculated to compensate for the field gradient due to the $\mathrm{C}$ magnet asymmetry, and changing the slope changes the quadrupole component of the field, with much smaller changes in the sextupole field and smaller-still changes in the higher poles. On the other hand, moving the iron wedges in or out radially changes the dipole component of the field while having little effect on the higher multipoles. There are 24 iron wedges for each $10^{\circ}$ section of the storage ring; moving all 24 wedges from one extreme position to changes the dipole field by $300 \mathrm{ppm}$, and the effect of moving just one wedge has a typical half-width of $10^{\circ}$ [50].

The surface coils, mounted on printed circuit boards, are constructed of $2 \times 120$ wires, each separated by $2.5 \mathrm{~mm}$, running azimuthally around the ring [49]. The coils are used to correct the lowest multipoles to tens of ppm. Dipole correction coils were placed in the air gaps between each $10^{\circ}$ pole, but were never used.

The magnet was shimmed using an iterative process of mapping the magnetic field and adjusting the edge and wedge shims to improve the field homogeneity. During these stages of development, a "shimming trolley" was used to map the field around the ring. This trolley is similar to that used to map the field inside the vacuum chamber during data taking (see Section 2.5.3), however since the vacuum chamber sections had not been constructed at the time, the shimming trolley rode on top of the pole pieces instead of inside the vacuum chamber. Furthermore, the magnetic field beyond the storage aperture was measured by the shimming trolley, which consists of 25 NMR probes and covers a wider cross-sectional area than the trolley used to measure the magnetic field during regular data taking. The POISSON[52] computer program was then used to calculate the necessary positions and widths of the wedge and edge shims to reduce both the azimuthal fluctuations of the dipole and to reduce the higher multipoles. The shimming process extended intermittently over several years, with the last changes made before the 2000 data run.

\subsubsection{Muon Injection and Beam Dynamics}

A full top schematic of the $g$-2 storage ring is shown in Fig. 2.7. At the exit of the inflector, the muon/pion beam sits $77 \mathrm{~mm}$ away from the central design orbit of the storage ring. In 


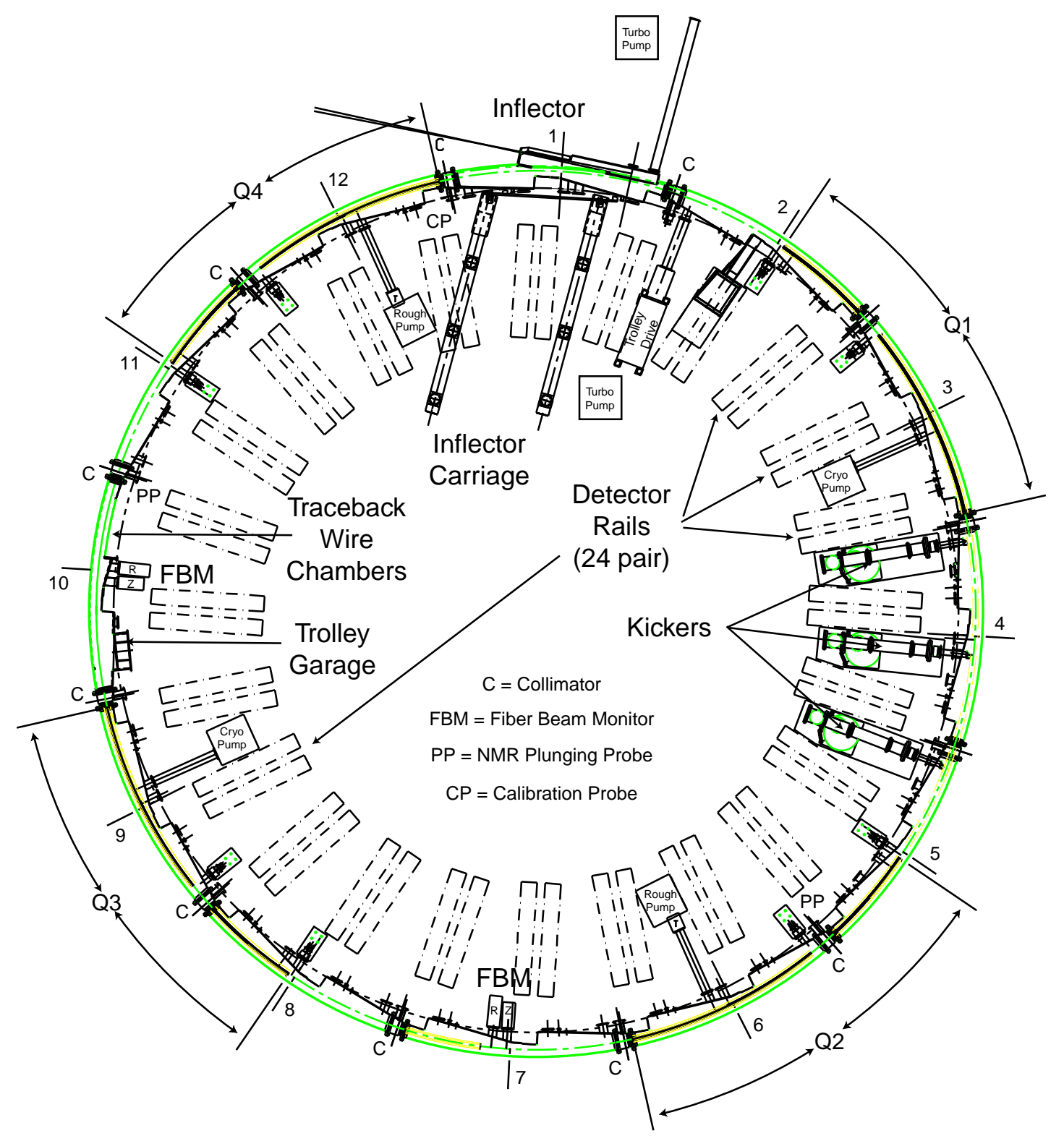

Figure 2.7: Top view of the $g-2$ storage ring. Three muon kicker modules are located $\sim 90^{\circ}$ away from the the inflector (injection point) and four electrostatic quadrupole regions (Q1Q4) vertically focus the beam. 


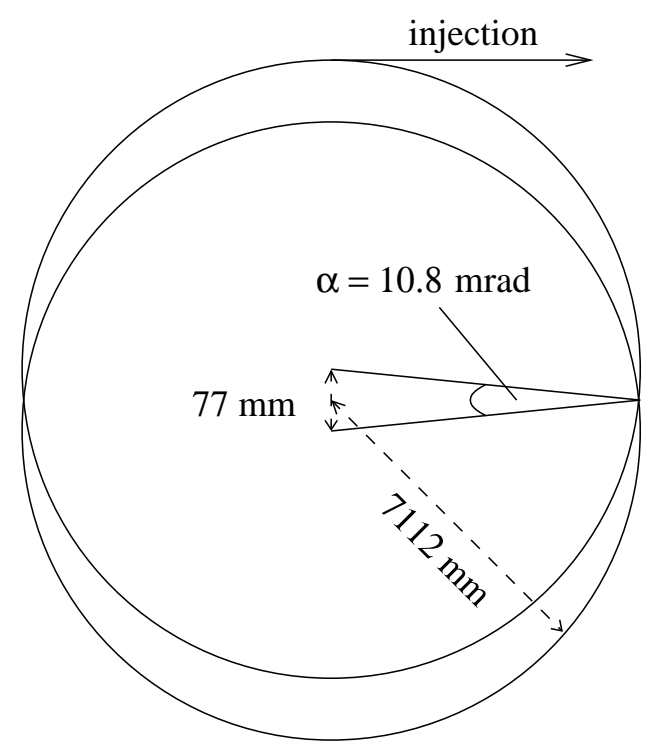

Figure 2.8: Schematic of muon injection. Muons are injected $7.7 \mathrm{~cm}$ from the center of the storage ring, and must be kicked $\simeq 10.8 \mathrm{mrad}$ outward onto the central orbit of the storage ring.

order to obtain stored muons, the beam is "kicked" onto a stable orbit by a fast, non-ferric, pulsed magnetic kicker. Four sets of pulsed electrostatic quadrupoles, symmetrically and discretely placed around the ring, provide vertical weak focusing of the beam.

\section{Muon Injection}

One of the main challenges in the BNL experiment is to store enough muons to obtain the desired statistical uncertainty in a reasonable amount of running time. As previously mentioned, a major difference between the current muon $g-2$ experiment at BNL and the CERN III experiment is the method of injecting muons into the storage ring. The CERN III experiment stored muons by first injecting pions into the storage ring. A very small fraction $\left(\sim 5 \times 10^{-5}\right)$ of these pions decayed with just the right phase space parameters so that the decay muons ended up in stable orbits. This approach not only yielded very few stored muons, but also produced large backgrounds which blinded the photomultiplier tubes mounted on their detectors, the so-called "flash". The flash forced the detectors to be gated on fairly late after injection, further reducing the number of detected decay positrons. A method was therefore developed of directly injecting muons into the E821 storage ring. This "muon injection" scheme results in a greater number of muons stored per AGS fill, and greatly reduces the flash in the detectors.

A schematic of the muon injection scheme is shown in Fig. 2.8. The beam is injected parallel but radially offset by $77 \mathrm{~mm}$ from the tangent of the central orbit of the storage ring, and since the radius of the central orbit is $7112 \mathrm{~mm}$, the beam requires a change ("kick") in its trajectory of $10.8 \mathrm{mrad}$ in the outward (positive) radial direction. An ideal 


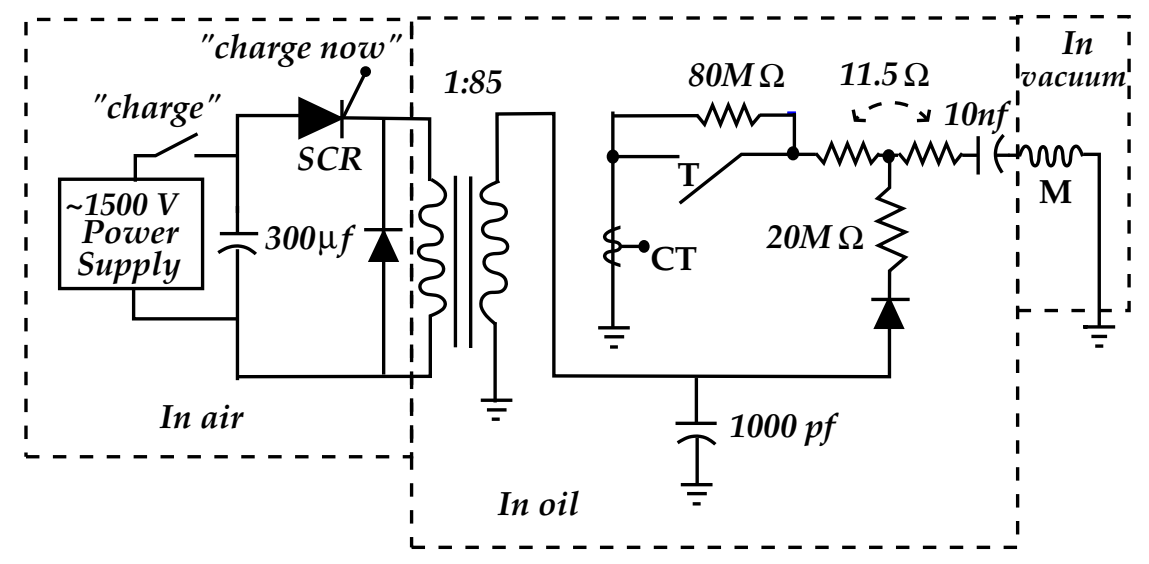

Figure 2.9: Circuit diagram of the "ideal" kicker electronics. The inductor labeled $\mathbf{M}$ represents the kicker magnet, $\mathbf{T}$ represents an English Electric Valve deuterium thyratron, and CT is a current transformer which gives the current waveform [53].

kick would be a delta function at exactly one quarter of the betatron wavelength (to be discussed in Section 2.4.2) of the injected muons. Since delta functions are a bit difficult to reproduce in the laboratory, the kick must be made over some distance along the muons' trajectory. For a beam momentum of $3.094 \mathrm{GeV} / \mathrm{c}$ and a magnetic field of $1.45 \mathrm{~T}$, one finds that $\mathrm{a} \sim 0.1 \mathrm{~T} \mathrm{~m}$ kick is required. Simulation shows such a kick results in more than an order of magnitude increase in the number of stored muons over the previously-used pion injection method.

Various options for how to kick the beam were considered, and there were several constraints that made the design of the kicker challenging. In order to maintain the homogeneity of the magnetic field of the storage ring, the kick cannot produce large eddy currents in the vacuum chamber walls (which in turn produce small local inhomogeneous magnetic fields), nor may magnetic materials be used. Furthermore, the length of the kicker is limited by the space between the electrostatic quadrupoles, about $5 \mathrm{~m}$. With these limitations, a fast, pulsed, non-ferric magnetic kicker design was chosen.

Three contiguous modules, each consisting of a pulse-forming network and two thin $\mathrm{Al}$ parallel plates $1.76 \mathrm{~m}$ long that sit just outside the storage aperture of the ring, provide about 4200 A peak current running in opposite directions along the plates. This current produces a magnetic field which partially cancels the local magnetic field of the storage ring and extends the radius of curvature such that the muons are directed onto stable orbits. The pulse-forming network is itself simply a LCR circuit, as shown in Fig. 2.9. The current on the plates is of the form

$$
I(t)=I_{0} e^{-\gamma t / 2} \sin \left(2 \pi f_{d} t+\phi_{d}\right)
$$

where

$$
f_{d}=\frac{1}{2 \pi} \sqrt{\frac{1}{L C}-\frac{R^{2}}{4 L^{2}}}
$$




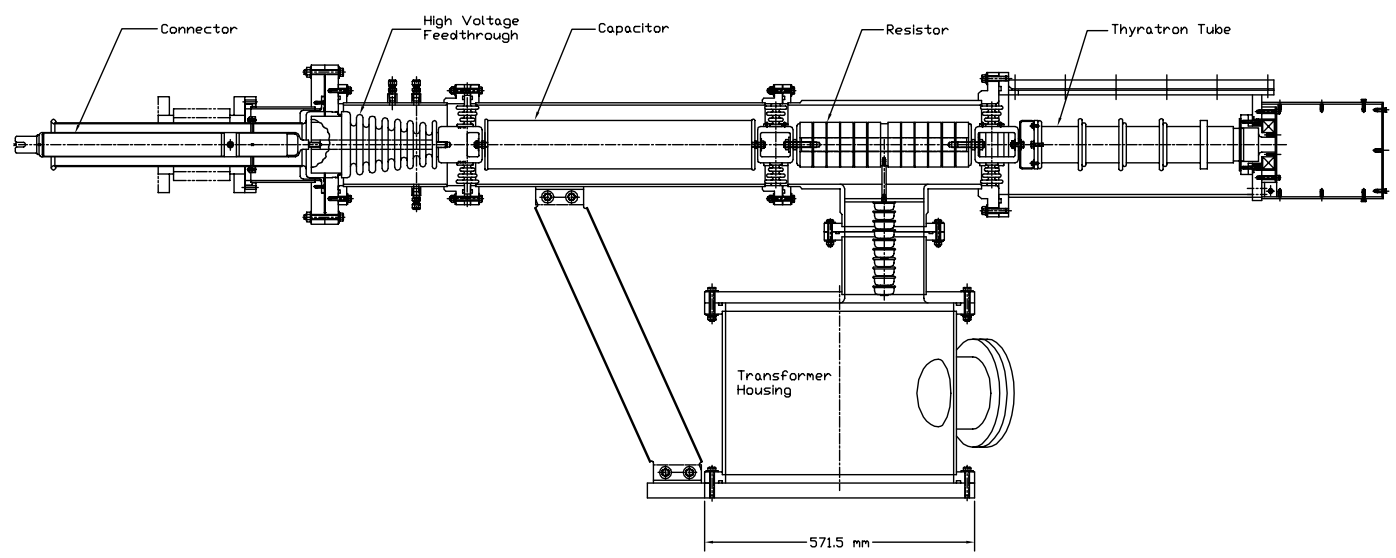

Figure 2.10: Side view of a kicker module. The connector carries the high current to the kicker-plates.

$$
\gamma=\frac{R}{L}
$$

and

$$
I_{0}=\frac{V_{0}}{2 \pi f_{d} L}
$$

The design values of the resistance and capacitance of the circuit are $11.5 \Omega$ and $10 \mathrm{nF}$. The estimated inductance of the circuit is $\sim 1.6 \mu \mathrm{H}$. These parameters result in a decay time of the waveform of $\sim 248 \mathrm{~ns}$. Since the cyclotron period is only $\sim 150 \mathrm{~ns}$, the beam receives a small second and a smaller-still third kick.

In order to avoid dangerous sparking and overheating, the pulse-forming network sits in a vessel filled with Dow-Corning 561 silicon dielectric fluid [53], labeled "oil" in Fig. 2.9, and the HV feed-through extending from the fluid to vacuum connects the kicker magnet to the rest of the electronics. If there were a mechanical failure of the HV feed-through, the vacuum would be contaminated with oil. In order to minimize this risk, a buffer zone containing 3M FC40 fluorocarbon (fluorinert) is placed between the fluid vessel and the vacuum chamber. Fig. 2.10 shows a side view of a kicker module, and the locations of the resistor stack, capacitor, HV feed-through and connector. The kicker plates are located at the end of the connector. Fig. 2.11 shows a cross section of the kicker plates inside the vacuum. The plates, which sit $50 \mathrm{~mm}$ away from the beam center, are $80 \mathrm{~mm}$ high, 1760 $\mathrm{mm}$ long and $0.75 \mathrm{~mm}$ thick, and are secured in place by HV Macor standoffs.

\section{Focusing the Beam}

Once inside the storage aperture, the injected muons must somehow be vertically focused. Otherwise they would be quickly lost through the top or bottom of the storage ring. Horizontal focusing is, of course, provided by the vertical magnetic field. Vertical focusing is provided by electrostatic quadrupole fields inside the storage ring in four locations, symmetrically placed, covering roughly $43 \%$ of the total circumference of the ring. By symmetrically covering only part of the ring, room is left for other devices inside the storage ring, such 


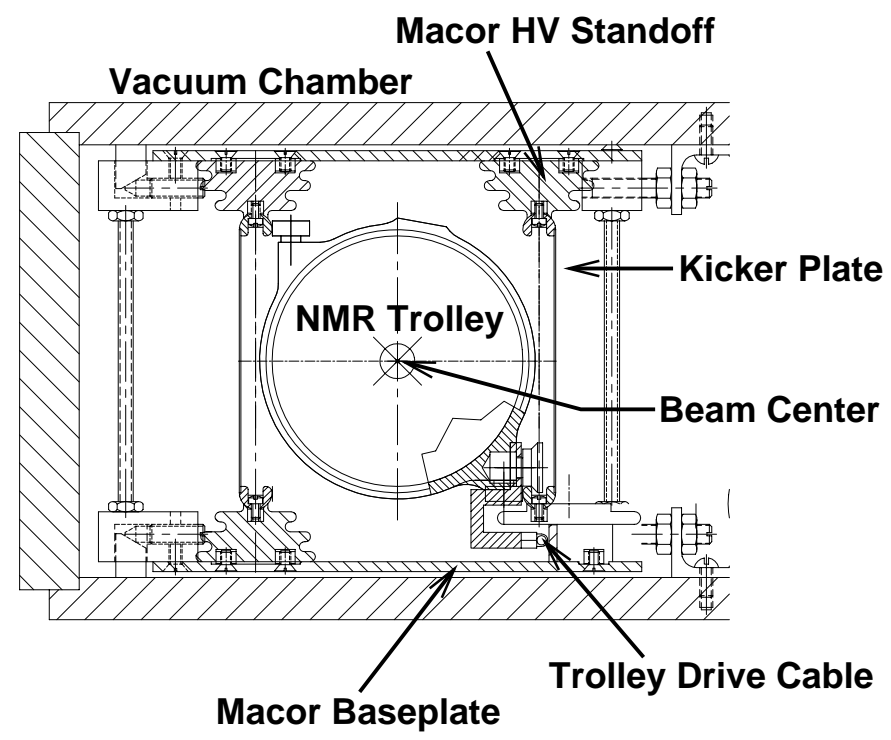

Figure 2.11: Cross-section view of the storage aperture in the kicker region.

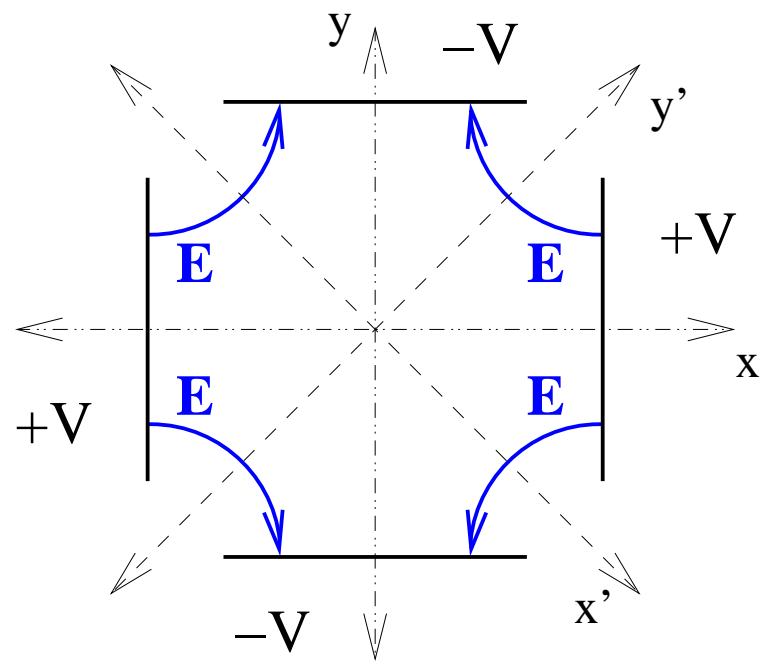

Figure 2.12: Quadrupole geometry: the four electrodes form a quadrupole electric field within the aperture of the storage ring. The axes $x^{\prime}$ and $y^{\prime}$ are along the electric field's symmetry lines, whereas the axes $x$ and $y$ are along the chosen coordinate system of $x, y$, and $s$. The symmetry of the field is shown in the solid blue lines connecting the plates. 
as the kicker and inflector. Unlike magnetic gradients, electrostatic focusing fields do not affect the azimuthal homogeneity of the magnetic field of the storage ring itself, and, as is discussed in Section 2.2, the effect of the electric fields on the spin precession frequency is eliminated to first order by choosing muons of the "magic momentum".

A cross-section schematic of the quadrupole electrodes is shown in Fig. 2.12. The solid curved lines represent the electric field. The $x$ and $y$ axes are along the chosen coordinate system of $x, y$, and $s$, where $x=0$ is the center of the storage aperture at a radius of 7.112 $\mathrm{m}, y=0$ is the vertical center of the storage ring, and $s$ is the azimuthal position in the storage ring. The $x^{\prime}$ and $y^{\prime}$ axes are along the electric field's symmetry lines, and it is trivial to show that

$$
E_{x^{\prime}}=\kappa y^{\prime}
$$

and

$$
E_{y^{\prime}}=\kappa x^{\prime}
$$

where

$$
\kappa=\frac{\partial E_{x^{\prime}}}{\partial y^{\prime}}
$$

Rotating to the chosen coordinate system of the $x$ and $y$ axes, we see that

$$
E_{x}=\kappa x
$$

and

$$
E_{y}=-\kappa y
$$

This simply shows that the electric field results in a restoring force in the vertical direction, and a repulsive force in the radial direction. In order to keep the beam focused, therefore, the restoring force of the vertical magnetic field must be stronger than the force from the electric field in the radial direction.

The Lorentz force is

$$
\vec{F}=q(\vec{E}+\vec{\beta} \times \vec{B})
$$

Therefore in the radial direction, we have

$$
F_{x}=\frac{\gamma m v^{2}}{r}-\frac{e}{c} v B_{y}+e E_{x}
$$

Noting that for a particle exactly on the equilibrium orbit $\left(r=R_{0}\right) E_{x}=0$ and therefore

$$
\frac{\gamma m v^{2}}{R_{0}}=\frac{e}{c} v B_{y}
$$

Also, we can rewrite $r^{-1}$ such that

$$
\frac{1}{r}=\frac{1}{R_{0}+x} \simeq \frac{1}{R_{0}}\left(1-\frac{x}{R_{0}}\right)
$$


Substituting 2.33 and 2.34 into 2.32 , we find that

$$
F_{x}=-e \beta B_{y}(1-n) \frac{x}{R_{0}}
$$

where $n$ is the field index defined by

$$
n=\frac{\kappa R_{0}}{\beta B_{y}}
$$

For the vertical motion, we have

$$
F_{y}=-e \kappa y
$$

The equations of motion are therefore

$$
\gamma m \ddot{x}+\frac{\gamma m v^{2}}{R_{0}^{2}}(1-n) x=0
$$

and

$$
\gamma m \ddot{y}+e \kappa y=0
$$

Eqs. 2.38 and 2.39 represent simple harmonic motion with the frequencies $f_{x_{\mathrm{BO}}}=f_{c} \sqrt{1-n}$ and $f_{y_{\mathrm{BO}}}=f_{c} \sqrt{n}$ respectively, where $f_{c}=v /\left(2 \pi R_{0}\right)$ is the cyclotron frequency. This motion is also known as betatron oscillation (hence the "BO" subscripts).

The above equations describe the motion of a single particle in both magnetic and electric fields. However as mentioned above, in this experiment, the electrostatic quadrupoles have a lattice design, with four-fold symmetry covering $43 \%$ of the ring. In this case, the equations for $x$ and $y$ as a function of azimuthal position in the ring are [54]

$$
x=x_{e}+\sqrt{\epsilon_{x} \beta_{x}(s)} \cos \left[\nu_{x} \frac{s}{R}+\phi_{x}\right]
$$

and

$$
y=\sqrt{\epsilon_{y} \beta_{y}(s)} \cos \left[\nu_{y} \frac{s}{R}+\phi_{y}\right]
$$

where $x_{e}=R_{e}-R_{0}$ is the equilibrium position in the storage aperture, $\epsilon_{x, y}$ are amplitude factors for the trajectories of individual particles, and $\beta_{x, y}(s)$ are the so-called Beta functions, which are periodic. $\nu_{x}$ and $\nu_{y}$ are defined as [54]:

$$
\begin{aligned}
\cos \left(\pi \nu_{x} / 2\right)= & \cos \left(l_{i} / R\right) \cos \left(\sqrt{1-n_{0}} l_{q} / R\right)- \\
& \frac{1}{2}\left(\frac{2-n_{0}}{\sqrt{1-n_{0}}}\right) \sin \left(l_{i} / R\right) \sin \left(\sqrt{1-n_{0}} l_{q} / R\right)
\end{aligned}
$$

and

$$
\cos \left(\pi \nu_{y} / 2\right)=\cos \left(\sqrt{n_{0}} l_{q} / R\right)-\frac{\sqrt{n_{0}} l_{i}}{2 R} \sin \left(\sqrt{n_{0}} l_{q} / R\right)
$$

where $n_{0}$ is the field index inside the quadrupoles, $l_{i}$ is the length of the interval between quadrupoles and $l_{q}$ is the length of a quadrupole. Therefore, $n_{0}=n / 0.43, l_{i} / R_{0} \simeq 0.90$ and $l_{q} / R_{0} \simeq 0.68$ in this experiment. However, since the four-fold symmetry period $\pi R / 2$ 


\begin{tabular}{|c|c|c||c|c|c|}
\hline $\begin{array}{c}\text { Order of } \\
\text { multipole }\end{array}$ & $\begin{array}{c}\text { Cosine Term } \\
\text { (Normal) }[\mathrm{V}]\end{array}$ & $\begin{array}{c}\text { Sine Term } \\
\text { (Skew) }[\mathrm{V}]\end{array}$ & $\begin{array}{c}\text { Order of } \\
\text { multipole }\end{array}$ & $\begin{array}{c}\text { Cosine Term } \\
\text { (Normal) }[\mathrm{V}]\end{array}$ & $\begin{array}{c}\text { Sine Term } \\
\text { (Skew) }[\mathrm{V}]\end{array}$ \\
\hline 1 & 0.0 & 0.0 & 8 & -5.5 & -0.2 \\
2 & 20177.8 & 0.1 & 9 & 0.0 & 0.0 \\
3 & 0.0 & 0.0 & 10 & -391.3 & 0.1 \\
4 & 33.0 & 0.1 & 11 & 0.0 & 0.0 \\
5 & 0.0 & 0.0 & 12 & -6.5 & 0.0 \\
6 & -45.9 & 0.1 & 13 & 0.0 & 0.0 \\
7 & 0.0 & 0.0 & 14 & 52.3 & -0.1 \\
\hline
\end{tabular}

Table 2.1: The multipole terms at $r=4.5 \mathrm{~cm}$, the edge of the muon storage region, for negative muon storage and $\pm 24 \mathrm{kV}$ on the plates.

is much less than the period of the radial oscillations $(\sim 2 \pi R)$ and even smaller than the vertical oscillations $(\sim 6 \pi R)$, we can in many cases use the field index $n$ averaged over the orbit $(n=\langle n(s)\rangle)$ to make estimates. This results in betatron tunes $\nu_{x}=f_{x_{\mathrm{BO}}} / f_{c}=\sqrt{1-n}$ and $\nu_{y}=f_{y_{\mathrm{BO}}} / f_{c}=\sqrt{n}$.

Since the field lines of a pure quadrupole field are hyperbolic, the ideal electrodes would also be hyperbolic in shape. However, in order to improve the positioning accuracy and lower manufacturing costs, each of the four quadrupole sections is made up of three $13^{\circ}$ lengths, each length made of 4 flat electrode plates. Flat plates obviously produce higher normal multipoles than hyperbolic plates, in particular the 12- and 20-poles. However, the 12-pole can be made near-zero by adjusting the width of the electrode, leaving the relative strength of the 20-pole to be $\sim 2 \%$ that of the quadrupole. Table 2.1 lists the normal and skew components of the electric field for flat plates held at $\pm 24 \mathrm{kV}$.

The electrode plates are made of $0.5 \mathrm{~mm}$ thick grade 5052-H34 aluminum and mounted on aluminum support frames using Macor electrical isolators. The placement of the quadrupole plates was confirmed to be within $\pm 0.5 \mathrm{~mm}$ of their design location $(50 \mathrm{~mm}$ away from the center of the storage volume) by a special survey taken in July of 1998 [54].

Each of the four quadrupole regions in the ring occupies $39^{\circ}$, the support frames installed in two $28^{\circ}$ vacuum chambers (Fig. 2.13(a)). The upstream chamber contains one $13^{\circ}$ segment, the other two segments sit in the downstream chamber. Because the quadrupole lengths could not be accurately placed in the bellows sections which connect the vacuum chambers to each other, there is a $4^{\circ}$ separation between the first and second lengths.

Field emission from the high voltage quadrupole plates produces free electrons, which can become trapped in the quadrupole regions. These trapped electrons oscillate about because of the presence of $E-$ and $B$-fields, which in turn could effect the determination of the magnetic field, since the trapped electrons are not present during the magnetic field measurement (the quadrupoles are turned off during the measurement of the magnetic field). Trapped electrons also accumulate on the quadrupole plate support insulators, which can result in sparking between the insulator and the positively charged vertical plate. To prevent sparking, the quadrupoles are pulsed, the duration of which is $\simeq 1 \mathrm{~ms}$. To further reduce the field emission, the quadrupoles were "conditioned" by applying $\sim 20 \%$ higher voltage than normal for short periods of time. The integrated maximum $B$-field disturbance from residual trapped electrons oscillating in the storage ring was determined [54] to be $0.03 \mathrm{ppm}$ 


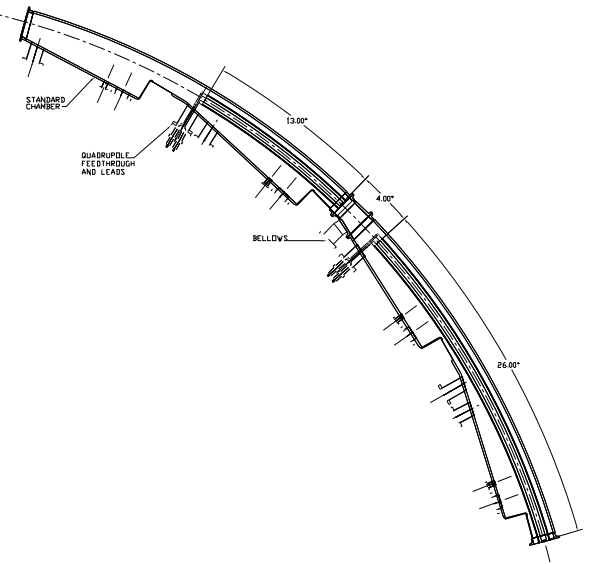

QUADRUPGLE REGION - TYPICAL

(a) Top view.

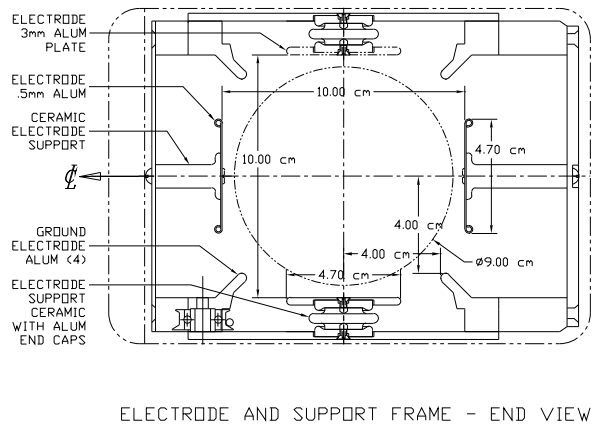

(b) Cross-section view.

Figure 2.13: Top and cross-section views of the electrostatic quadrupoles inside the $g-2$ storage ring.

of the storage ring $B$-field $(\simeq 1.45 \mathrm{~T})$.

\section{Resonance, Muon Losses and Scraping}

Resonances occur when particles repeatedly (synchronously) pass through perturbations in the focusing fields. As a result, a stored beam becomes unstable and particles may be lost. In this experiment, lost muons produce a non-exponential background in the decay electron time spectrum. It can be easily shown [55] that the most general resonance condition for both horizontal and vertical motion is

$$
k \nu_{x}+l \nu_{y}=n
$$

where $k, l$, and $n$ are integers and $\nu_{x}$ and $\nu_{y}$ are the betatron tunes defined above. Since for our focusing scheme,

$$
\nu_{x}^{2}+\nu_{y}^{2} \simeq 1
$$

the effective $n$-value must be chosen with care to avoid resonances. Eq. 2.45 is not exact because of the four-fold symmetry of the quadrupoles in the storage ring; the sum of the squares is in fact slightly $\left(\sim 2 \times 10^{-3}\right)$ larger than 1 . Fig. 2.14 shows an area of the tune plane encompassing the typical $n$-values used in this experiment. The various resonance lines are drawn, as is the curve in Eq. 2.45. The two operating $n$-values for the 2001 data set are also shown, $n=0.122$ and $n=0.142$. We note that in 1999 and 2000 data were collected using $n \simeq 0.137$. However, this resulted in a rather large systematic error (to be discussed later) in the determination of $\omega_{a}$. Therefore in 2001 it was decided to take data at two different $n$-values in order to decrease the sensitivity of $\omega_{a}$ to the $n$-value. 


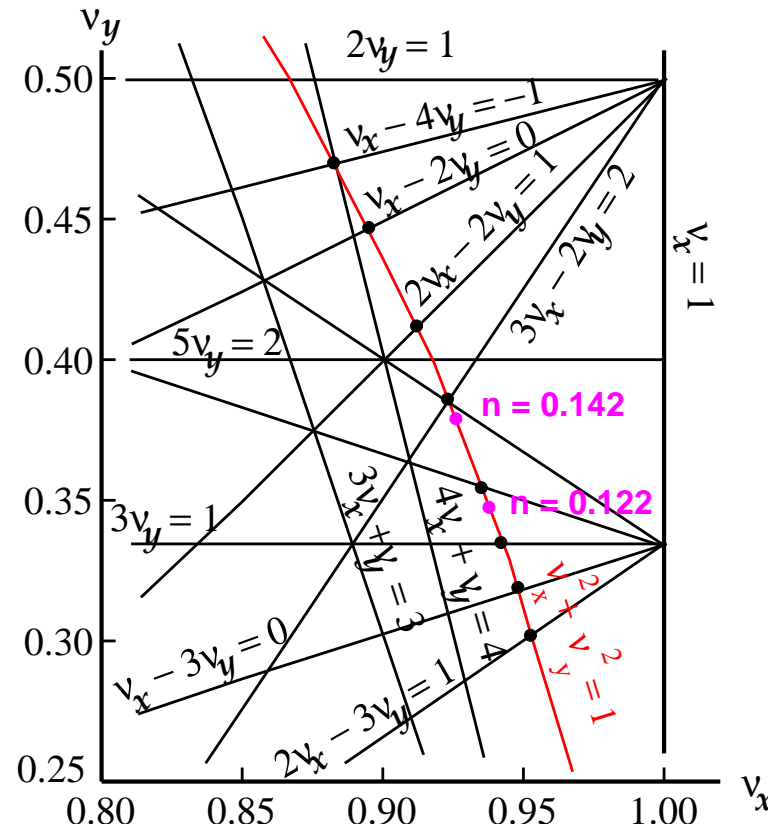

Figure 2.14: Tune plane in the region near the operating values of the $g$-2 storage ring.

Beam losses cannot, however, be entirely avoided in the experiment. Therefore, to further reduce the number of lost muons, at early times after injection $(\sim 0-25 \mu \mathrm{s})$ the beam is "scraped" against the edges of $3 \mathrm{~mm} \mathrm{Cu}$ rings with an inner radius of $4.5 \mathrm{~cm}$ and an outer radius of $5.5 \mathrm{~cm}$. By adding differences in the voltages on the quadrupole plates (effectively adding dipole terms to the electric field), the beam center is pushed vertically down $2 \mathrm{~mm}$ and radially out $2 \mathrm{~mm}$ on one side of the ring and radially in $2 \mathrm{~mm}$ on the other side of the ring. Those particles that sit on the edge of the allowed phase-space of the storage ring, and are therefore more likely to be lost at later times, clip the edge of the collimators, lose energy, are scattered and quickly lost. After some time (15 $\mu$ s in 2000, 7 $\mu \mathrm{s}$ in 2001), scraping is slowly turned off with a RC time constant of $5 \mu \mathrm{s}$.

\section{Coherent Betatron Motion}

Because of the mismatch between the inflector and storage ring apertures, the phase-space of the storage ring is not uniformly filled. Therefore a beam "image" is created in phase-space $\left(\left(x, x^{\prime}\right)\right.$ or $\left.\left(y, y^{\prime}\right)\right)$ at the exit of the inflector and is re-formed every integer and half-integer betatron wavelength (the image is inverted at half-integer betatron wavelengths). This beam motion is referred to as coherent betatron oscillation (CBO).

Fig. 2.15 illustrates this effect: at a particular position and time $\left(s=\right.$ Det. A,t $\left.t=t_{1}\right)$, the beam has a given phase-space distribution. One cyclotron period later, at $(s=$ Det. $\mathrm{A}, t=t_{1}+T_{c}$ ), the phase-space distribution of the beam appears different, and in fact the same distribution as found at $\left(s=\right.$ Det. $\left.\mathrm{A}, t=t_{1}\right)$ does not re-form until the beam reaches another location, in this example that of detector $\mathrm{B}$, at $\left(s=\right.$ Det. $\left.\mathrm{B}, t=t_{1}+t_{\mathrm{BO}}\right)$. The phase-space distribution therefore "walks" around the ring, so at a given location in the 


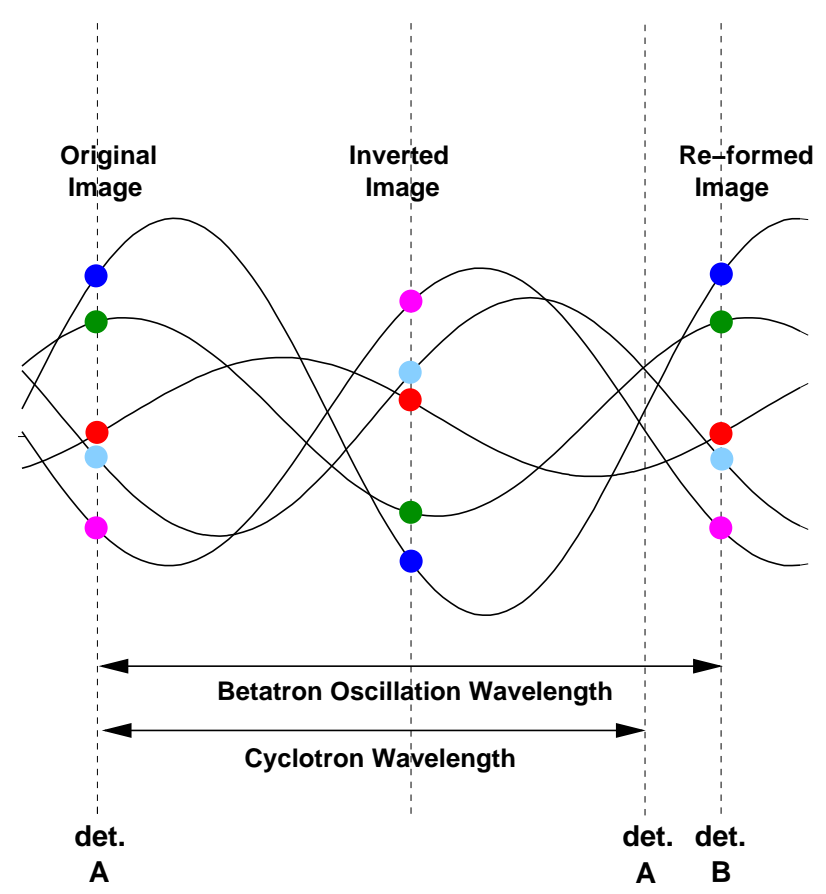

Figure 2.15: Illustration of coherent betatron motion. The solid curves represent the betatron oscillations of individual muons. Since the betatron oscillation period is longer than the cyclotron period, the phase-space distribution at detector A varies with time.

ring, the phase-space distribution re-forms at the CBO frequency:

$$
\omega_{x_{C B O}}=\omega_{c}-\omega_{x_{B O}}=\omega_{c}(1-\sqrt{1-n})
$$

and

$$
\omega_{y_{C B O}}=\omega_{c}-\omega_{y_{B O}}=\omega_{c}(1-\sqrt{n})
$$

In this experiment, the situation is complicated somewhat by the kick the muons receive upon injection. Fig. 2.16 shows the time-dependence of the radial phase-space distribution, $\left(x, x^{\prime}\right)$. For clarity, the kick is treated as a perfect delta function, however, as in the case of the actual experiment, the beam is not kicked to the exact center of the acceptanceellipse (defined by the maximum and minimum values of $x$ and $x^{\prime}$ allowed by the storage ring). Therefore the radial beam centroid and width oscillate inside the ring. Vertical coherent betatron oscillations also occur, but the kick does not affect the vertical phase-space distribution. Coherent betatron oscillations result in time-dependent acceptance effects, which will be discussed in Section 4.3.1.

\section{The Radial Electric Field Correction}

To first order the effect of using electric fields to focus the beam on the spin precession frequency is canceled by injecting muons at the "magic" momentum such that $\gamma=\gamma_{m}=$ $1+1 / a_{\mu}$. However, the storage ring has a momentum acceptance of $\pm 0.5 \%$ and the injected 


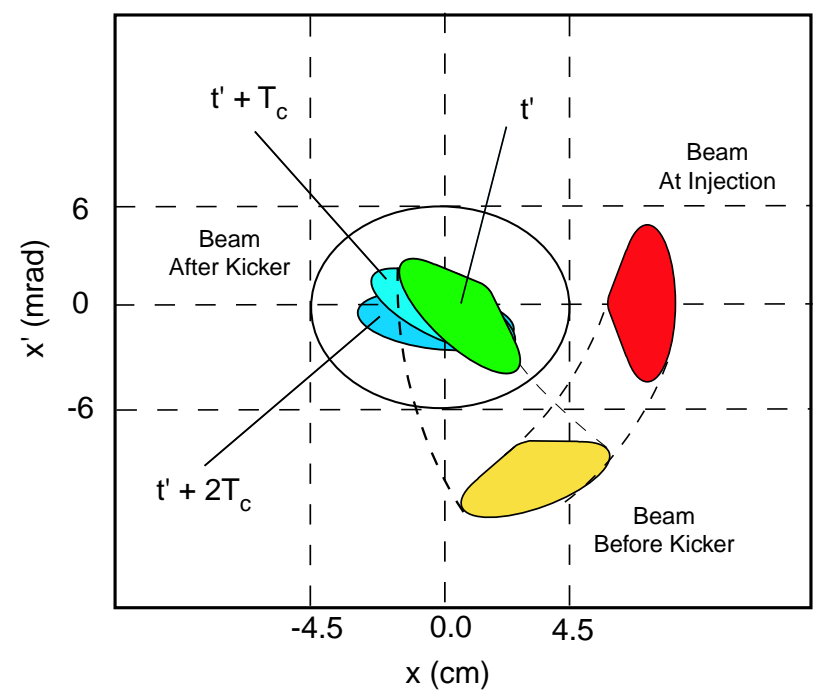

Figure 2.16: Schematic of the radial phase-space diagram of the beam at injection, just before the kick and just after the kick. The image of the beam at the inflector rotates around inside the storage ring acceptance-ellipse as a function of time.

beam is not mono-energetic. As a result, the observed spin precession frequency is reduced by the presence of the $\vec{\beta} \times \vec{E}$ terms in Eq. 2.8 for off-magic-momentum muons stored in the ring. The following second-order correction, called the Radial Electric Field Correction $\left(C_{E}\right)$, must be applied to the measured spin precession frequency.

For a muon of momentum $p \neq p_{m}$, the spin precession frequency is

$$
\omega_{a}^{\prime}=\omega_{a}\left[1-\beta \frac{E_{r}}{B_{y}}\left(1-\frac{1}{a_{\mu} \beta^{2} \gamma^{2}}\right)\right]
$$

where $E_{r}$ is the radial electric field and $B_{y}$ is the vertical magnetic field. Substituting $p=\beta \gamma m=\left(p_{m}+\Delta p\right)$, we find

$$
\begin{aligned}
\frac{\Delta \omega_{a}}{\omega_{a}} & =-\frac{\beta E_{r}}{B_{y}}\left[1-\frac{m^{2}}{a_{\mu} p_{m}^{2}\left(1+2 \frac{\Delta p}{p_{m}}+\left(\frac{\Delta p}{p_{m}}\right)^{2}\right)}\right] \\
& \simeq-\frac{\beta E_{r}}{B_{y}}\left[1-\frac{m^{2}\left(1-2 \frac{\Delta p}{p_{m}}-\left(\frac{\Delta p}{p_{m}}\right)^{2}\right)}{a_{\mu} p_{m}^{2}}\right]
\end{aligned}
$$

Since $m^{2} / a_{\mu} p_{m}^{2}=1$, we find that

$$
\frac{\Delta \omega_{a}}{\omega_{a}}=-2 \frac{\beta E_{r}}{B_{y}}\left(\frac{\Delta p}{p_{m}}\right)
$$


Using the relation

$$
\frac{\Delta p}{p}=(1-n) \frac{\Delta R}{R_{0}}=(1-n) \frac{x_{e}}{R_{0}}
$$

where $x_{e}$ is the particle's equilibrium radius relative to the center of the aperture of the storage ring, and

$$
E=\kappa x=n \beta B_{y} \frac{x}{R_{0}}
$$

we find

$$
\frac{\Delta \omega_{a}}{\omega_{a}}=-2 n(1-n) \beta^{2} \frac{x x_{e}}{R_{0}^{2} B_{y}}
$$

Assuming a perfect quadrupole field, the time-average of $x$ for a given particle is simply $x_{e}$ since the horizontal oscillations are perfectly sinusoidal. Since $\tau_{\mu} \gg T_{x_{\mathrm{BO}}}$ (the period of horizontal betatron oscillations), replacing $x$ with $x_{e}$ is perfectly acceptable. Thus for each muon,

$$
C_{E}=\frac{\Delta \omega_{a}}{\omega_{a}}=-2 n(1-n) \beta^{2} \frac{x_{e}^{2}}{R_{0}^{2} B_{y}}
$$

The average value of $C_{E}$ depends on the average square of the equilibrium radius, $\left\langle x_{e}^{2}\right\rangle$, which must be either computed or, preferably, measured.

\section{The Vertical Pitch Correction}

Another second-order correction that must be applied to the measured value of $\omega_{a}$ is the so-called Vertical Pitch Correction $\left(C_{P}\right)$. Eq. 2.9 assumes $\vec{\beta} \cdot \vec{B}=0$, yet the stored muons have a vertical velocity component upon injection and the weak-focusing fields force the muons to oscillate up and down inside the storage ring. This motion results in a smaller magnetic force felt by the muons, thus a reduced observed spin precession frequency.

A general derivation of the vertical pitch correction may be found in [43]. Here we present a derivation for the pitch correction for this specific experiment. We assume $\vec{B}=\left(0, B_{y}, 0\right)$, $\vec{\beta}=\left(\beta_{x}, \beta_{y}, \beta_{z}\right), \beta_{x, y} / \beta_{z} \ll 1$, and $a_{\mu}=1 /\left(\gamma^{2}-1\right)$. Hence, all $\vec{\beta} \times \vec{E}$ are neglected.

We consider a reference frame in which the momentum vector always lies in the $y-z$ plane, as depicted in Fig. 2.17. The $x$ - and $z$-axes therefore rotate about the $y$-axis at a frequency

$$
\omega=\frac{e}{m \gamma} B_{y}
$$

The pitch angle $\psi$ oscillates at the vertical betatron frequency $\omega_{1}=\omega_{B O_{y}}$ with an amplitude $\psi_{0}$ :

$$
\psi=\psi(t)=\psi_{0} \cos \omega_{1} t
$$

As shown in Fig. 2.17, the spin precession frequency is resolved into components parallel and perpendicular to the plane $P$. However the parallel component changes in sign and thus the net effect (time average) of the parallel component is zero. The perpendicular component of $\omega_{a}$ is in this coordinate system

$$
\omega_{\perp}=\omega_{a}=\omega_{y} \cos \psi-\omega_{z} \sin \psi
$$




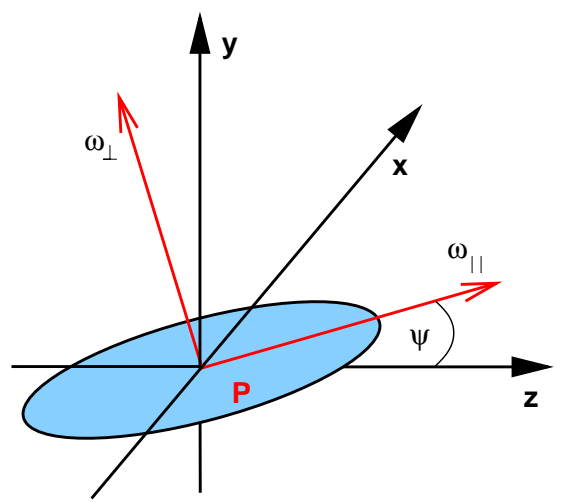

Figure 2.17: In a rotating coordinate system such that the momentum vector always lies in the $y-z$ plane, the vertical pitch angle $\psi$ oscillates at the vertical betatron oscillation frequency.

The components of the spin precession frequency vector are obtained using Eq. 2.8:

$$
\begin{aligned}
\omega_{a_{y}} & =\frac{e}{m} a_{\mu} B_{y}-\frac{e}{m} a_{\mu} B_{y}\left(\frac{\gamma}{\gamma+1}\right) \beta_{y}^{2} \\
& =\omega_{0}\left[1-\left(\frac{\gamma}{\gamma+1}\right) \beta_{y}^{2}\right] \\
& =\omega_{0}\left[1-\left(\frac{\gamma}{\gamma+1}\right) \beta^{2} \frac{\beta_{y}^{2}}{\beta^{2}}\right]
\end{aligned}
$$

Since

$$
\frac{\beta_{y}}{\beta}=\sin \psi \simeq \psi
$$

and

$$
\frac{\gamma}{\gamma+1} \beta^{2}=\frac{\gamma}{\gamma+1} \frac{\gamma^{2}-1}{\gamma^{2}}=\frac{\gamma-1}{\gamma}
$$

we have

$$
\omega_{a_{y}}=\omega_{0}\left[1-\left(\frac{\gamma-1}{\gamma}\right) \psi^{2}\right]
$$

Next we have

$$
\begin{aligned}
\omega_{a_{z}} & =-\frac{e}{m} a_{\mu} B_{y}\left(\frac{\gamma}{\gamma+1}\right) \beta_{y} \beta_{z} \\
& =-\omega_{0}\left(\frac{\gamma}{\gamma+1}\right) \beta^{2} \frac{\beta_{y}^{2}}{\beta^{2}} \frac{\beta_{z}}{\beta_{y}}
\end{aligned}
$$

Since

$$
\frac{\beta_{y}}{\beta_{z}}=\tan \psi \simeq \psi
$$


then

$$
\omega_{a_{z}}=-\omega_{0}\left(\frac{\gamma-1}{\gamma}\right) \psi
$$

Inserting Eqns. 2.61 and 2.64 into Eq. 2.57 and using the small angle approximation for $\psi$, we find

$$
\omega_{a}=\omega_{\perp} \simeq \omega_{0}\left(1-\frac{\psi^{2}}{2}\right)
$$

Therefore we find that to a very good approximation,

$$
C_{P}=\frac{\psi^{2}}{2}
$$

and the average value of $C_{P}$ depends on the average value of the square of the vertical pitch angle, $\left\langle\psi^{2}\right\rangle$, which must be computed or, preferably, measured.

\subsubsection{Monitoring the Muon Beam}

\section{Fast Rotation Analysis}

As discussed in Section 2.4.2, determining the average radial distribution of the stored muon beam is necessary for calculating the radial electric field correction to the spin difference frequency. An effective method for determining this distribution was developed for the second and third CERN muon $(g-2)$ experiments, based on the debunching of the beam at early times after injection. The time width of the beam $(\sim 22 \mathrm{~ns})$, is much smaller than the cyclotron period $(\sim 150 \mathrm{~ns})$. The bunched structure of the beam results in a modulation in the number of counts at the cyclotron frequency at very early times after injection. These high-frequency modulations are often referred to as the "fast-rotation" modulation.

Since all particles travel at essentially the same speed $(0.9994 \mathrm{c})$, those particles with smaller equilibrium radii will move steadily ahead of those particles with larger equilibrium radii. Therefore the time distribution broadens of over time at a rate which depends primarily on the initial equilibrium radius distribution. Fig. 2.18 shows the time spectrum of the decay electrons shortly after injection: the plot on the left shows a very large fast rotation amplitude at early times $(\sim 6-12 \mu \mathrm{s})$ after injection whereas the plot on the right shows much smaller amplitudes at later times $(\sim 36-42 \mu \mathrm{s})$ after extensive beam debunching.

A method to determine the average radial distribution of the stored muons developed for the CERN muon $g$-2 experiments involves an iterative fit to the increasing time-width of the circulating beam. An alternative method uses Fourier analysis of the same data [56]. Both methods agree quite well, as shown in Fig. 2.19. The values in Fig. 2.19(a) are with respect to the design orbit radius of $711.2 \mathrm{~cm}$. The mean radial positions of the beam as determined by the two methods agree within $5 \times 10^{-5}$, and the widths typically agree within $5 \%$.

\section{Traceback Chambers}

Another indirect means of measuring the stored muon distribution is by tracking the paths of decay electrons. The point of decay of a muon is determined by the measurement of 


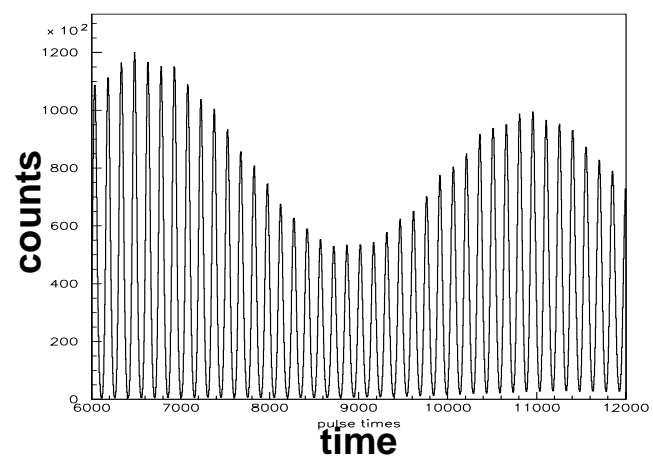

(a)

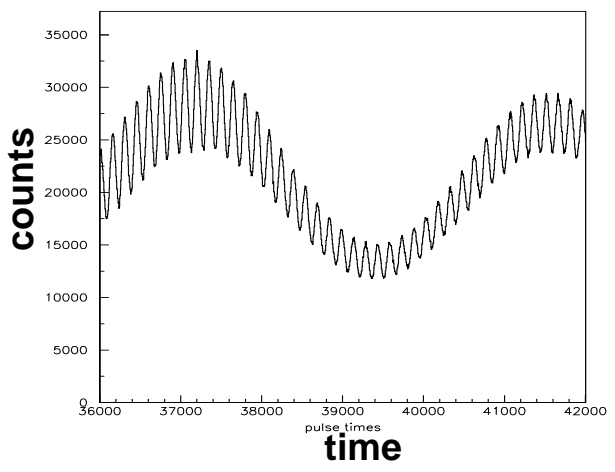

(b)

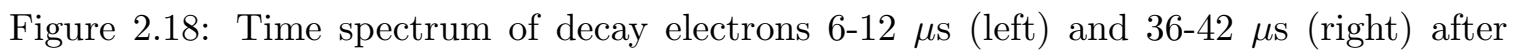
injection. The short time-scale ("fast rotation") oscillations are due to the structure of the bunched beam, and the long time-scale oscillations are due to the $g$-2 oscillation in the detected number of counts. Note the amplitude of the fast rotation oscillations dies away at later times as the beam de-bunches.

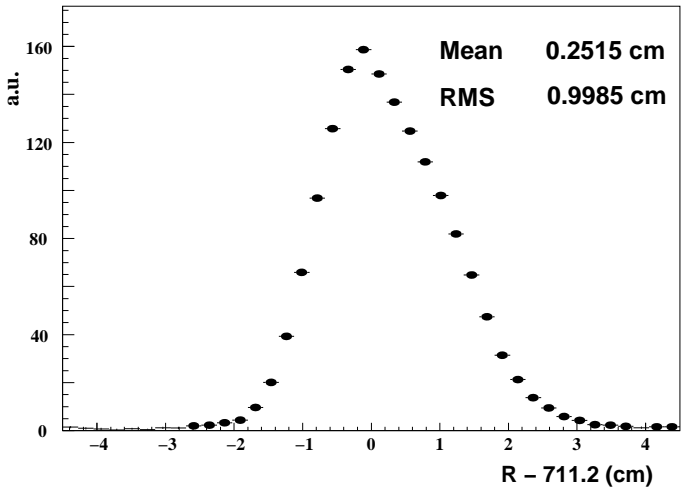

(a) Results of CERN method.

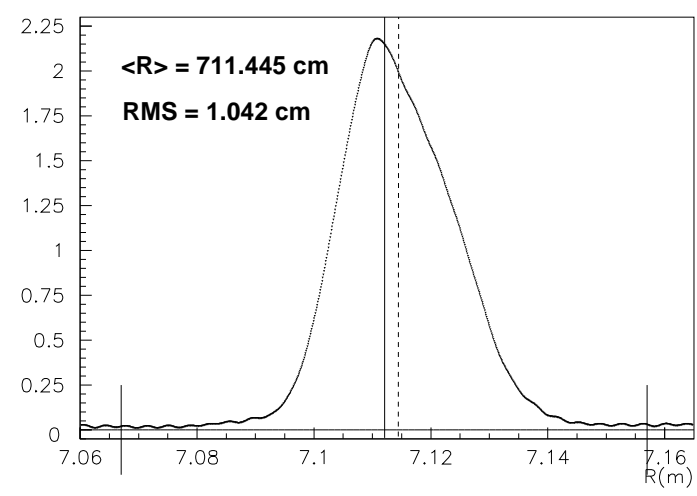

(b) Results of Fourier analysis approach.

Figure 2.19: Equilibrium radial distributions obtained using two different fast-rotation analyses. 

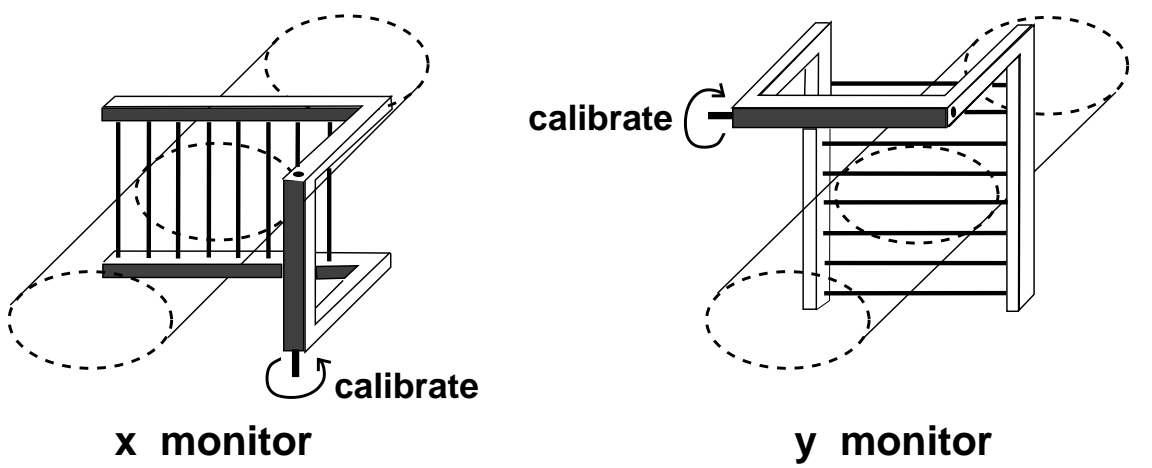

Figure 2.20: Sketch of the fiber beam monitors.

the radius of curvature of the path of the decay electron, assuming the decay electron is emitted tangentially with respect to the equilibrium orbit (which is true, on average). The distribution of decay vertices is directly related to the distribution of stored muons.

The paths of decay electrons are measured using a series of four straw tube "traceback" wire chambers located just upstream of an electromagnetic calorimeter (detector number 20), which is located far away from the scalloped vacuum wall[51]. Each traceback chamber consists of a pair of radially and vertically aligned sets of straws. Decay electrons produce ionization signals in the straws. The paths of decay electrons, and hence their point of decay, may be reconstructed (or "traced back") from signals from contiguous straws and chambers. Unfortunately, although traceback data were successfully collected during the 1999 and 2000 data runs, the chambers failed to respond in 2001 and no data were obtained for the latest data run.

\section{Fiber Beam Monitors}

A more direct means of measuring the beam motion is via fiber beam monitors (FBMs), shown in Fig. 2.20. A FBM consists of seven, thin, parallel scintillating fibers, running either vertically or radially; the fibers are strung through an aluminum structure which may be placed in the storage aperture of the ring when FBM data is taken. Otherwise the structure sits above the storage aperture, away from the beam.

Four such detectors were built for this experiment, two x-y beam monitor pairs located $\sim 180^{\circ}$ and $\sim 270^{\circ}$ away from the injection point. The light output of each individual fibers is directed into a photo-multiplier tube, the output of which is read out continuously by a waveform digitizer. Because of data acquisition system constraints, only the first $\sim 10 \mu \mathrm{s}$ of data could be taken after injection.

In order to calibrate the signals from each fiber, the FBMs may be put in "calibration mode", where all seven fibers are rotated such that all fibers see the same amount of beam (see Fig. 2.20). The signals from the fibers are compared to each other and normalized. Once the response of the fibers are calibrated, the FBMs are put in "data mode", where the seven fibers are rotated orthogonal to the beam. The time-dependence of the beam centroid and width can then be measured. The time and frequency spectra obtained from 

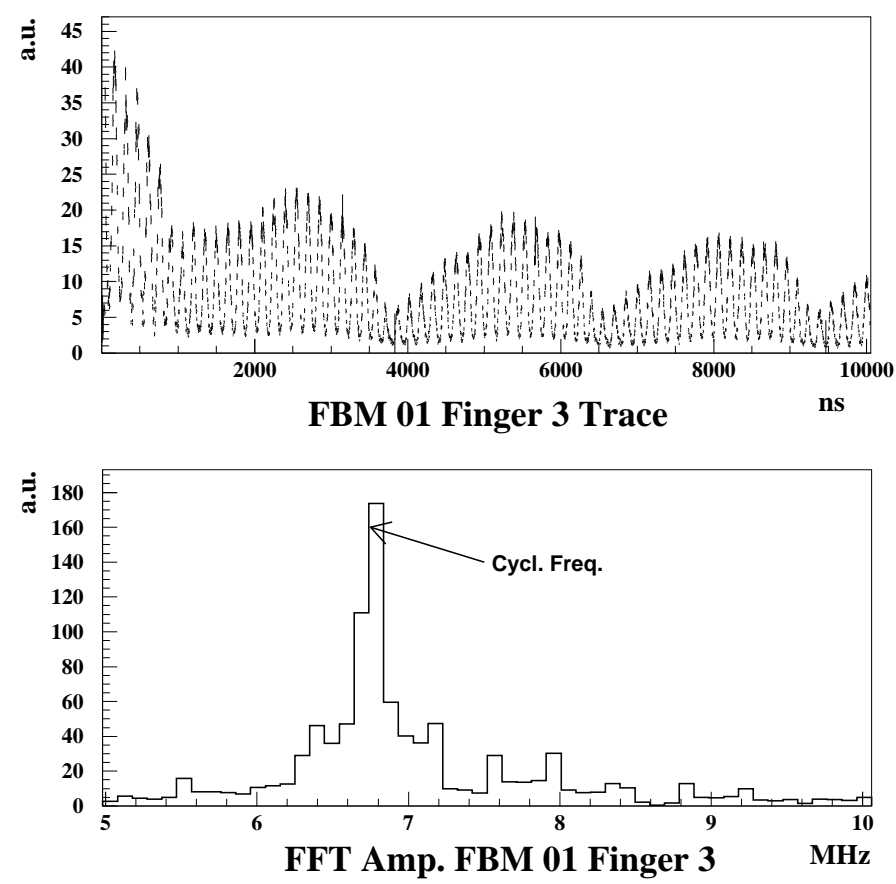

Figure 2.21: Time and frequency spectra of a single fiber read-out. The FBM is located at $\sim 180^{\circ}$ from the injection point and data are read out for the first $\sim 10 \mu$ s after injection. The high-frequency oscillations are due to the fast-rotation, the low-frequency oscillations (seen only in the top plot) are due to coherent radial beam motion.

calibration data for a single fiber are shown in Fig. 2.21, where we see both a high- and a low-frequency modulation of the time spectrum. The high frequency corresponds to the fast-rotation; the bunched beam produces signals in the scintillating fibers as it passes by the FBM. The low frequency corresponds to the radial CBO. As the beam moves in and out radially, it moves back and forth across the individual finger. Plots of the measured radial CBO are shown in Section 4.3.1.

\subsubsection{Muon Injection and Beam Dynamics Simulation}

Although various means exist to measure the muon distribution inside the storage ring, further insight can be obtained from Monte Carlo simulation. A muon tracking simulation named g2track, originally written in Fortran by S. Mane and consequently modified by E. Efstathiadis and others, integrates the equations of motion in small steps around the $g$-2 storage ring. One could certainly write a tracking code using faster beam dynamics/optics techniques. However, these techniques are designed to transport particles over large distances and are therefore unable (at least not efficiently) to take into account fluctuations in a magnetic field to the order of one ppm. Using g2track, however, one can get a microscopic view of the effects on the stored muon distribution of perturbations in the $\vec{E}$ and $\vec{B}$ fields, which we have in the form of detailed electrostatic quadrupole field calculations and precise measurements of the $g$-2 magnetic field. 


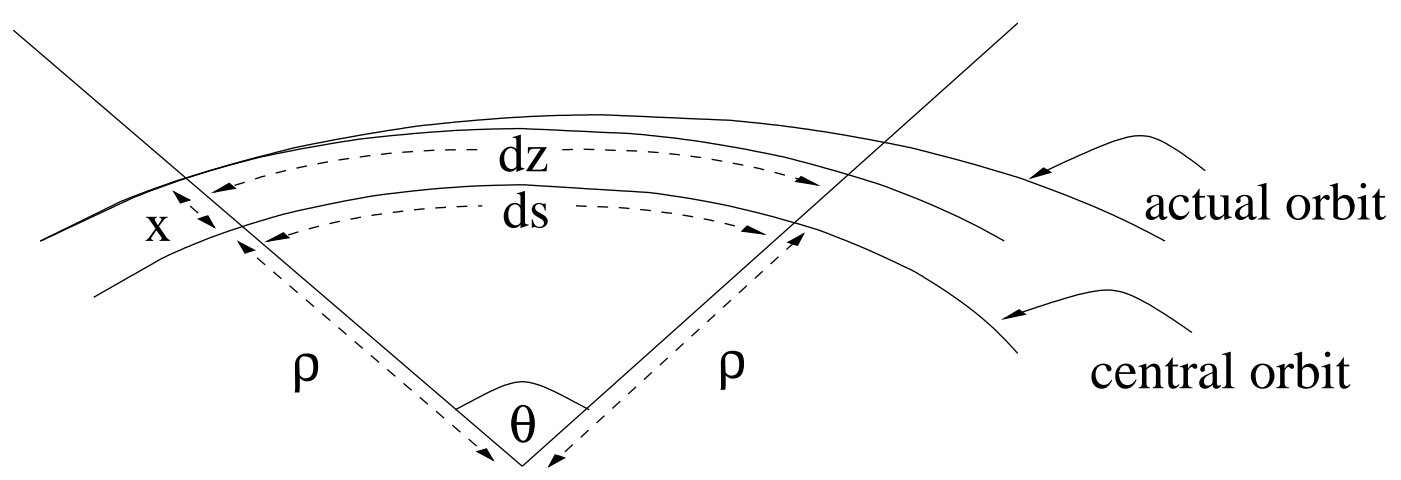

Figure 2.22: Coordinate system used in g2track.

\section{The Equations of Motion}

We are used to seeing equations of motion in terms of derivatives with respect to time. However, in g2track it is more useful to think in terms of azimuthal position, so the equations of momentum are a bit trickier to derive. The coordinate system used in the simulation is shown in Fig. 2.4.4. $+\hat{x}$ points radially out, $+\hat{y}$ points vertically up, and $+\hat{s}$ is tangential to the central (design) orbit at the current azimuthal position.

To derive our equations of motion, we begin with

$$
\vec{F}(t)=\frac{d \vec{p}}{d t}
$$

which we need to rewrite as $\vec{F}(s)$. Now,

$$
\begin{aligned}
\vec{p}(s) & =\vec{p}\left(s_{0}\right)+\int_{t\left(s_{0}\right)}^{t(s)} \overrightarrow{F(t) d t} \\
& =\vec{p}\left(s_{0}\right)+\int_{s_{0}}^{s} \vec{F}\left(s^{\prime}\right) \frac{d t}{d s^{\prime}} d s^{\prime} \\
& =\vec{p}\left(s_{0}\right)+\int_{s_{0}}^{s} \vec{F}\left(s^{\prime}\right) t^{\prime} d s^{\prime}
\end{aligned}
$$

where $t^{\prime}=d t / d s$.

However, in going from $s_{0}$ to $s$, our coordinate system was rotated around the y-axis by an angle

$$
\theta=\int_{s_{0}}^{s} h\left(s^{\prime}\right) d s^{\prime}
$$

where $\mathrm{h}(\mathrm{s})$ is the curvature of the orbit (in other words, $1 / \rho(s)$ ). So, we need to rotate $p(s)$ to our new "local" $p, p_{\text {local }}$ :

$$
\begin{aligned}
\vec{p}_{\text {local }}(s) & =\vec{R}(\theta) \cdot \vec{p}(s) \\
& =\vec{R}(\theta) \cdot\left(\vec{p}\left(s_{0}\right)+\int_{s_{0}}^{s} \vec{F}\left(s^{\prime}\right) t^{\prime} d s^{\prime}\right)
\end{aligned}
$$


where $\vec{R}(\theta)$ is the rotation matrix:

$$
\vec{R}(\theta)=\left(\begin{array}{ccc}
\cos \theta & 0 & \sin \theta \\
0 & 1 & 0 \\
-\sin \theta & 0 & \cos \theta
\end{array}\right)
$$

Now,

$$
\begin{aligned}
\vec{F}(s) & =\frac{d \vec{p}_{\text {local }}(s)}{d s} \\
& =\frac{d}{d s} \vec{R}(\theta) \cdot\left(\vec{p}\left(s_{0}\right)+\int_{s_{0}}^{s} \vec{F}\left(s^{\prime}\right) t^{\prime} d s^{\prime}\right)
\end{aligned}
$$

From Eq. 2.71, we see that

$$
h(s)=\frac{d \theta}{d s}
$$

so that

$$
\frac{d \vec{R}(\theta)}{d s}=\left(\begin{array}{ccc}
-h(s) \sin \theta & 0 & h(s) \cos \theta \\
0 & 1 & 0 \\
-h(s) \cos \theta & 0 & -h(s) \sin \theta
\end{array}\right)
$$

which, in the limit $\theta \rightarrow 0$, gives us

$$
\frac{d \vec{p}_{\text {local }}(s)}{d s}=\vec{I} \cdot \vec{F}(s) t^{\prime}+\left(\begin{array}{ccc}
0 & 0 & h(s) \\
0 & 0 & 0 \\
-h(s) & 0 & 0
\end{array}\right) \cdot p(s)
$$

where $\vec{I}$ is the identity matrix.

We can now write our equations of motion. Since

$$
\vec{F}(s)=q(\vec{E}+\vec{\beta} \times \vec{B})
$$

we find that

$$
\begin{aligned}
& p_{x}^{\prime}=\frac{d p_{x}}{d s}=q E_{x} t^{\prime}+q\left(\beta_{y} B_{z}-\beta_{z} B_{y}\right) t^{\prime}+h p_{z} \\
& p_{y}^{\prime}=\frac{d p_{y}}{d s}=q E_{y} t^{\prime}+q\left(\beta_{z} B_{x}-\beta_{x} B_{z}\right) t^{\prime} \\
& p_{z}^{\prime}=\frac{d p_{z}}{d s}=q E_{z} t^{\prime}+q\left(\beta_{x} B_{y}-\beta_{y} B_{x}\right) t^{\prime}-h p_{x}
\end{aligned}
$$

We also need expressions for $x^{\prime}=d x / d s, y^{\prime}=d y / d s$, and $z^{\prime}=d z / d s$. Looking at Fig. 2.4.4 we obtain the following relations:

$$
z^{\prime}=\frac{d z}{d s}=\frac{\rho+x}{\rho}=1+h x
$$

where $h=1 / \rho$ 


$$
x^{\prime}=\frac{d x}{d s}=\frac{d x}{d z} \frac{d z}{d s}=(1+h x) \frac{p_{x}}{p_{z}}
$$

Similarly, we get:

$$
y^{\prime}=\frac{d y}{d s}=(1+h x) \frac{p_{y}}{p_{z}}
$$

and so

$$
\begin{aligned}
\frac{d t}{d s} & =\frac{1}{v} \sqrt{\left(\frac{d x}{d s}\right)^{2}+\left(\frac{d y}{d s}\right)^{2}+\left(\frac{d z}{d s}\right)^{2}} \\
t^{\prime} & =\frac{(1+h x)}{v} \frac{p_{t}}{p z}
\end{aligned}
$$

where $p_{t}=\sqrt{p_{x}^{2}+p_{y}^{2}+p_{z}^{2}}=$ total momentum. Eqs. 2.81-2.83 and 2.84-2.88 will be numerically integrated in g2track.

\section{Integration Routine}

There are of course several different numerical algorithms to integrate ordinary differential equations. Perhaps the best known is the Runge-Kutta method, which is robust, will work on most ordinary differential equations (ODEs), but will produce low-accuracy solutions. Since our equations of motion are quite smooth functions, the Bulirsch-Stoer method is preferable. This algorithm calculates the values of the variables using a few different step sizes, and take the limit as the step size approaches zero. It is considered the fastest and most accurate routine known for the integration of smooth ODEs (see [57], for details), and is implemented in g2track using the CERNLIB library routine DDEQBS.

\section{Simulating The Kicker}

The $g$-2 kicker is simulated in g2track as close as possible to reality. Therefore, three "kickers", with physical specifications identical to the real kickers used in the experiment, are included in the g2track storage ring. The physics kickers in the simulation include threesets of two parallel kicker plates of the correct height, width and length. The simulation uses a multipole kicker magnetic field.

Beginning in the 1999 run, the kicker pulses of each module were read out by a WFD and placed into the data stream. Time-averaged kicker pulses are shown in Fig. 2.23. The kicker WFD readout data were then fit to obtain the values of $\mathrm{L}, \mathrm{C}$ and $\mathrm{R}$ for each kicker module. However, fitting the data to the simple equation for an over-damped harmonic oscillator resulted in too-large current values at later times (eg: after $800 \mathrm{~ns}$ ). Therefore a linear term was added to the fit function to bring this value down and give an improved parameterization of the kicker current (see Fig. 2.24):

$$
I(t)=\frac{I_{0}}{\omega L} e^{-X t} \sin (\omega t)+a t+b
$$



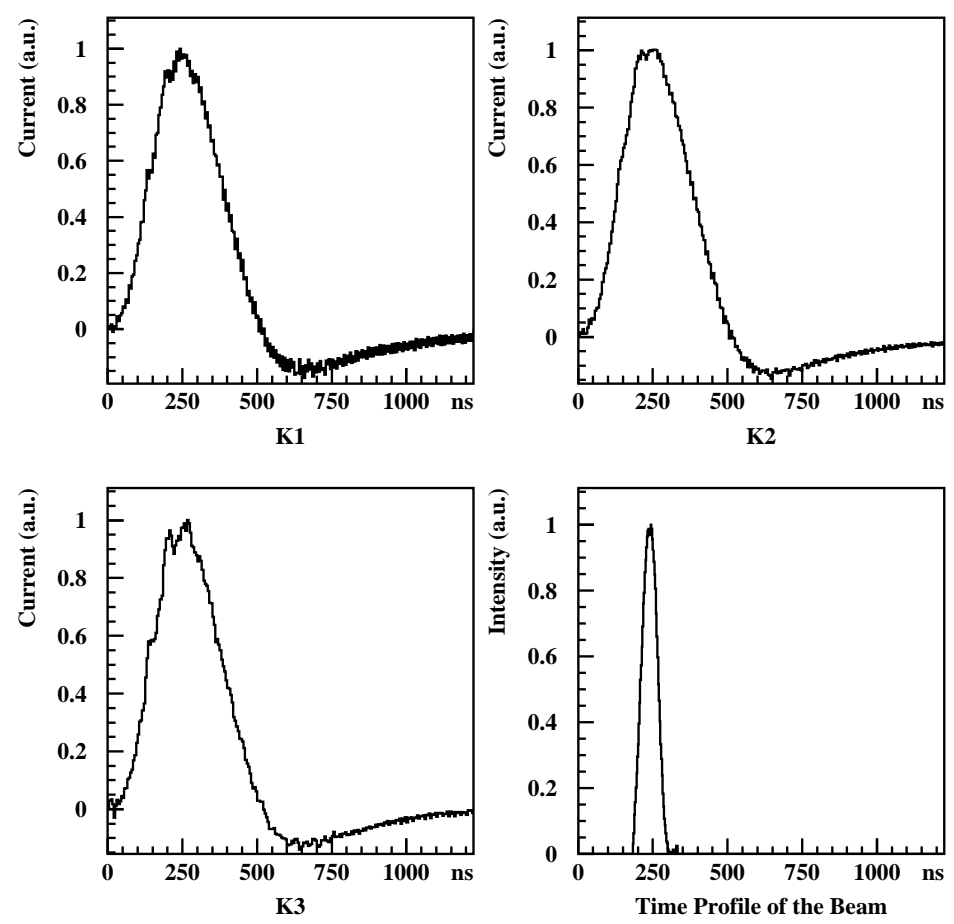

Figure 2.23: Average kicker pulse shapes and T0 pulse shape, as read from WFDs.

where $a t+b$ is the linear term,

$$
X=\frac{R}{2 L}
$$

and

$$
\omega=\sqrt{\frac{1}{L C}-X^{2}}
$$

Calculations of the time varying magnetic field were made using the OPERA 2d package[58] by Y. Semertzidis and incorporated into the earlier versions of g2track by E. Efstathiadis. The value of the current and each muon's position in the storage ring is then used in a multipole calculation of the magnetic field (see [59]).

\section{Simulating The Electrostatic Quadrupoles and Scraping}

As with the kicker modules, precise physical descriptions of the electrostatic quadrupoles are used in g2track. Four sets of four aluminum electrostatic quadrupole plates (top, bottom and sides of storage region), matching the size and locations of the real quadrupoles are evenly distributed around the ring. Multipole calculations of the electrostatic fields, both during and after scraping, were made by Semertzidis using POISSON (see, for example, [60]). In order to speed up the simulation, the electrostatic fields are obtained using a look-up table of grid width $1 \mathrm{~mm}$, and points in between are linearly interpolated. Edge and curvature effects of the quadrupole plates are taken into account by increasing the effective length of 


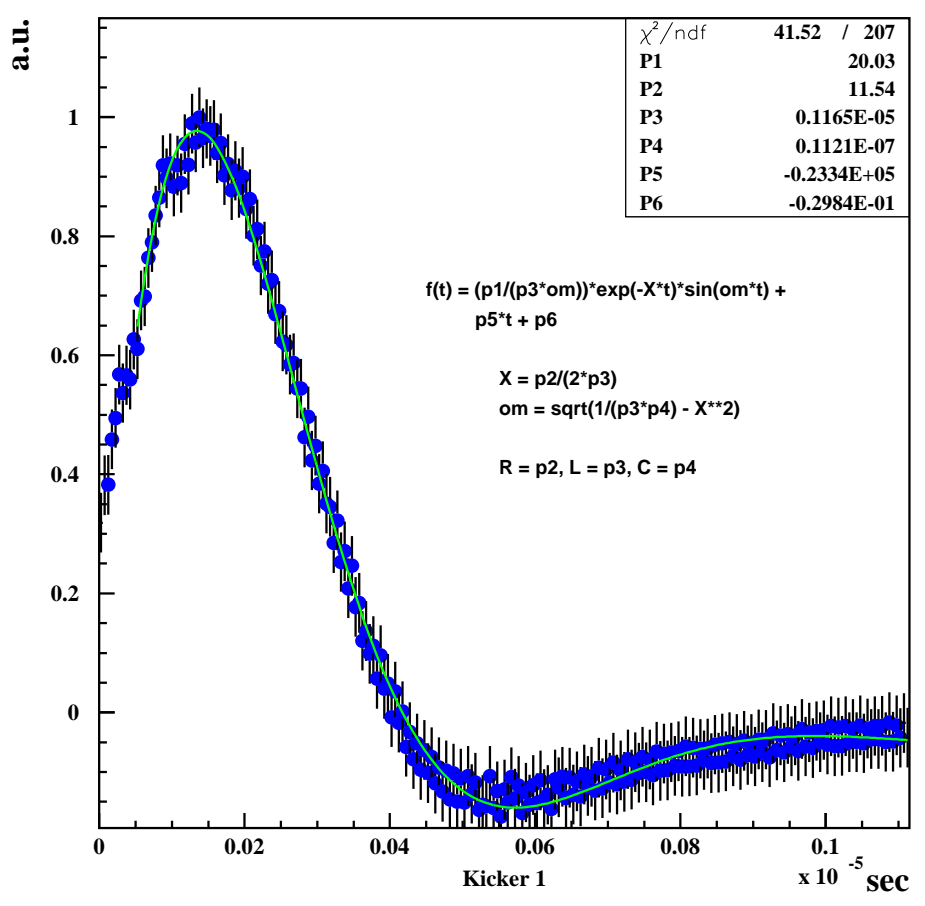

Figure 2.24: Simulated kicker current pulse used in g2track. The pulse is calculated using fitted values of $\mathrm{L}, \mathrm{C}$ and $\mathrm{R}$ of a LCR circuit to a real kicker pulse.

the quadrupoles and adding a curvature correction to the calculated fields. Scraping turns off with a linear interpolation between the scraping fields and non-scraping fields over 15 $\mu \mathrm{s}$. The amount of time the beam is scraped is a variable in the simulation, typically set to $7-15 \mu \mathrm{s}$.

\section{Input Muon Distribution}

The input muon distribution is obtained from the BTRAF simulation, which tracks particles from the point of the target all the way to the exit of the $g-2$ inflector. All beamline magnets are included in the simulation. Figs. 2.25-2.26 show the radial and vertical distribution of the injected muons, as well the radial and vertical phase space diagrams.

\section{Tracking Results}

Each muon created by BTRAF and successfully transported to the exit of the inflector is then transported step-by-step through the $g-2$ storage ring. Although by default the vertical magnetic field is held constant at $1.4513 \mathrm{~T}$ and there is no radial magnetic field, the user has the option to include a multipole map of the magnetic field. When the muon enters the kicker region or a quadrupole region it is subjected to the time-dependent magnetic and electric fields found in those regions.

Fig. 2.27 shows the phase-space diagram of a muon during its first two turns around the storage ring. The muon enters close to $7.8 \mathrm{~cm}$ outside the center of the storage ring; 


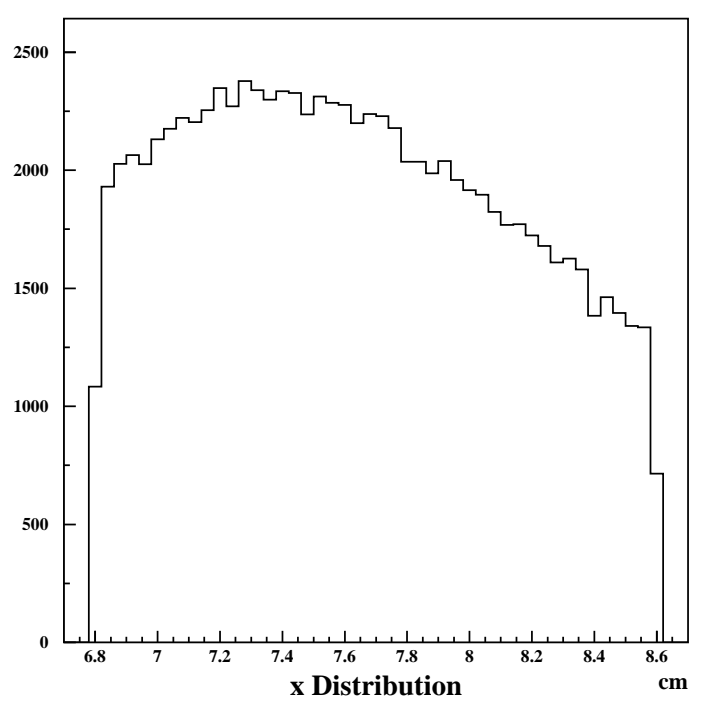

(a) Radial distribution.

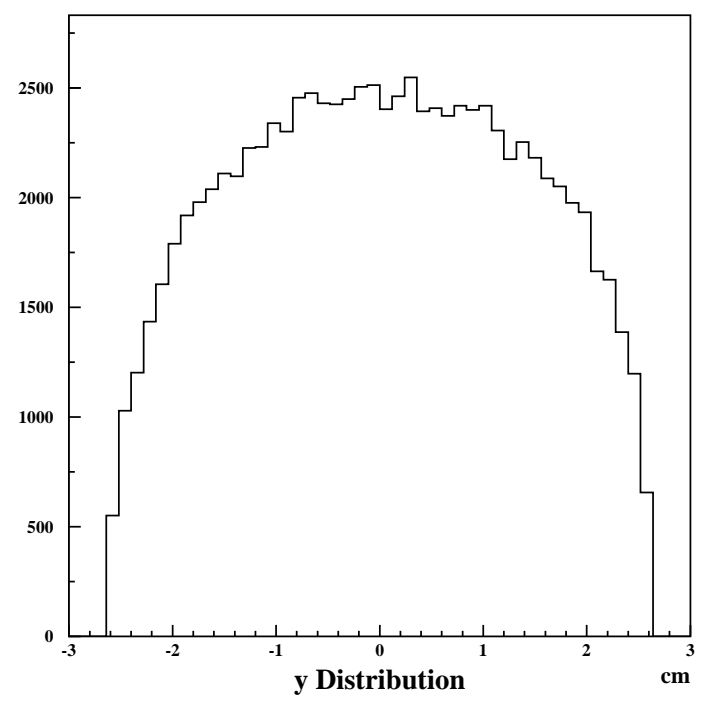

(b) Vertical distribution.

Figure 2.25: Injected muon radial and vertical distributions as obtained from BTRAF. The asymmetry of the radial distribution is due to the shape of the inflector (see Fig. 2.5(b)).

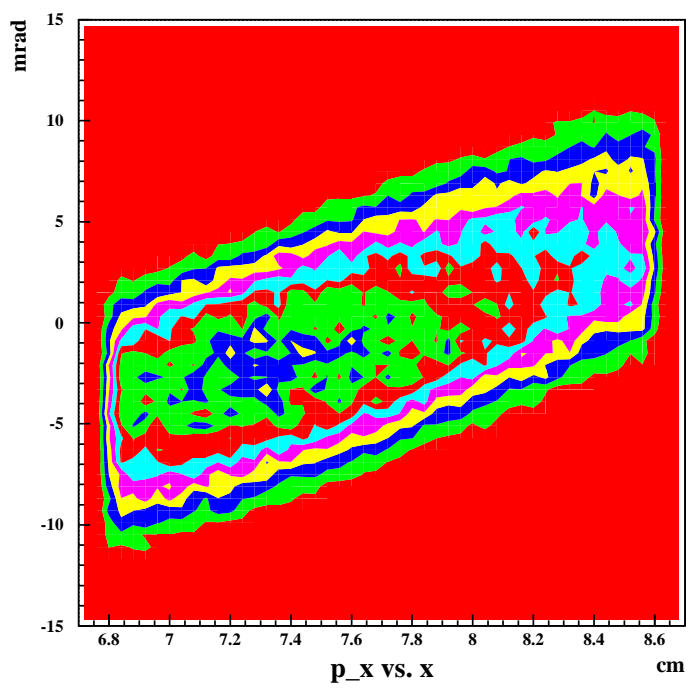

(a) Radial phase space distribution.

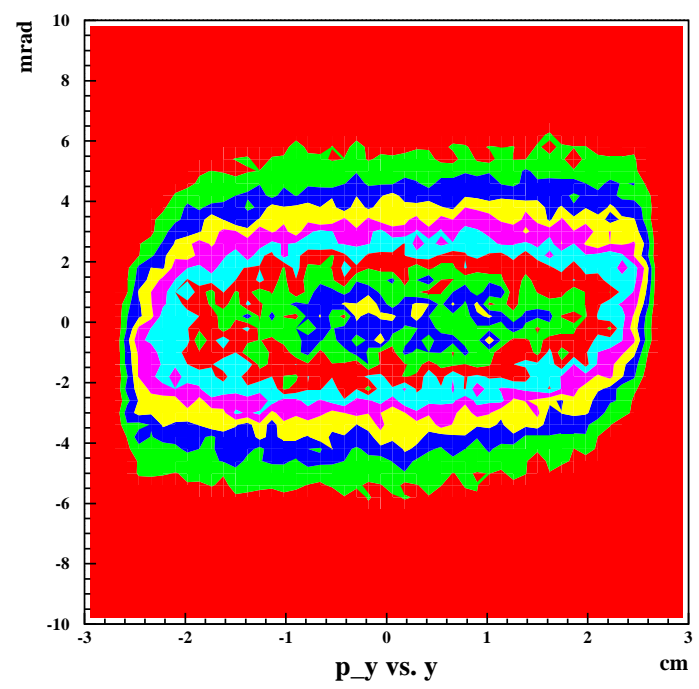

(b) Vertical phase space distribution.

Figure 2.26: Phase space distributions of the injected muons, as obtained from BTRAF. 


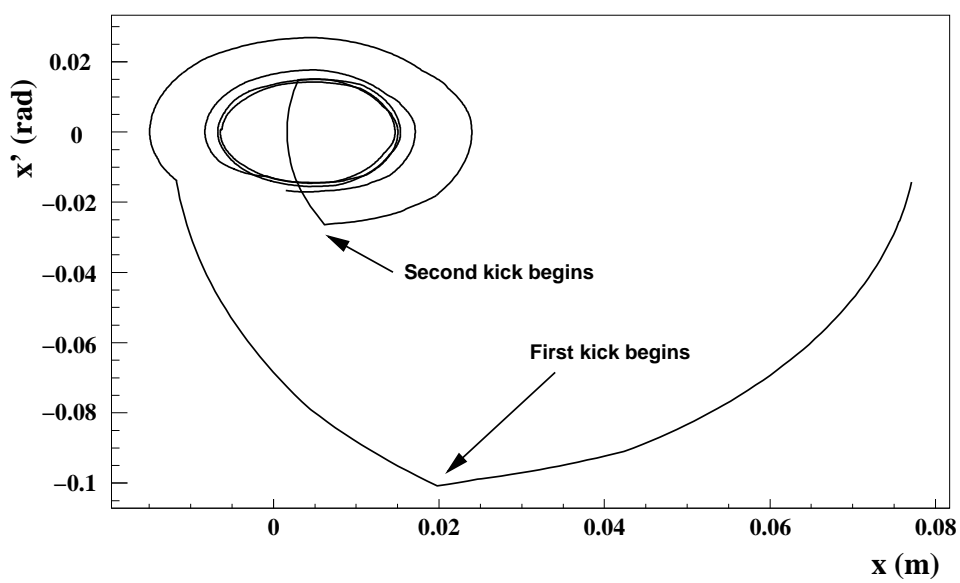

Figure 2.27: Phase-space diagram of $x$ vs. $x^{\prime}$ for a single muon in the g2track simulation. The muon is injected nearly $8 \mathrm{~cm}$ outside of the center of the storage aperture, and two kicks, the result of the kicker current pulses being wider than the cyclotron period, are seen.

near $90^{\circ}$ of the circumference of the ring later, the muon enters the kicker region, where we see the resulting kick in both $x$ and $x^{\prime}$. The oscillatory (circular) motion of $x$ vs. $x^{\prime}$ arises from the focusing electric fields. Note also that because the width of the kicker pulse is greater than twice the cyclotron period (see Fig. 2.23), the muon receives a second kicks. Therefore, although the timing of the kicker pulse is aligned such that the injected muons see the peak of the kicker pulse, they also receive smaller second kick (and indeed a much smaller third kick).

CBO is also easily seen using g2track. By looking at a single location in the ring (such as where the fiber beam monitors, or FBMs, sit), we can measure the radial centroid of the beam as a function of time. Fig. 2.28 shows the motion of the radial centroid of the beam as determined in g2track, for an $n$-value of $n \simeq 0.122$. The oscillations were fit to the function

$$
\langle x\rangle(t)=A_{\mathrm{CBO}} e^{-t / \tau_{\mathrm{CBO}}} \cos \left(2 \pi f_{\mathrm{CBO}} t+\phi_{\mathrm{CBO}}\right)+x_{e}
$$

where $A_{\mathrm{CBO}}(\mathrm{P} 1$ in Fig. 2.28$)$ is the amplitude of the $\mathrm{CBO}$ oscillation, $\tau_{\mathrm{CBO}}(\mathrm{P} 5)$ is the $\mathrm{CBO}$ lifetime, $f_{\mathrm{CBO}}(\mathrm{P} 2)$ is the $\mathrm{CBO}$ frequency, $\phi_{\mathrm{CBO}}(\mathrm{P} 3)$ is the phase of the $\mathrm{CBO}$ oscillations, and $x_{e}(\mathrm{P} 4)$ is the average radial beam position. The frequency agrees extremely well with the expected frequency, as we see that the fitted lifetime of the CBO is of the order $100 \mu \mathrm{s}$.

\section{Tracking of Lost Muons}

g2track is also well-suited to study lost muons. Since a multipole expansion of the electric field is used in the simulation, muons are indeed lost in g2track. Figure 2.29 shows a scan of the fraction of lost muons per muon lifetime over different quadrupole high-voltages used in g2track. The quadrupole voltages have been calibrated by comparing the radial CBO frequency found in the simulation to that found in the data. Two resonances are apparent 


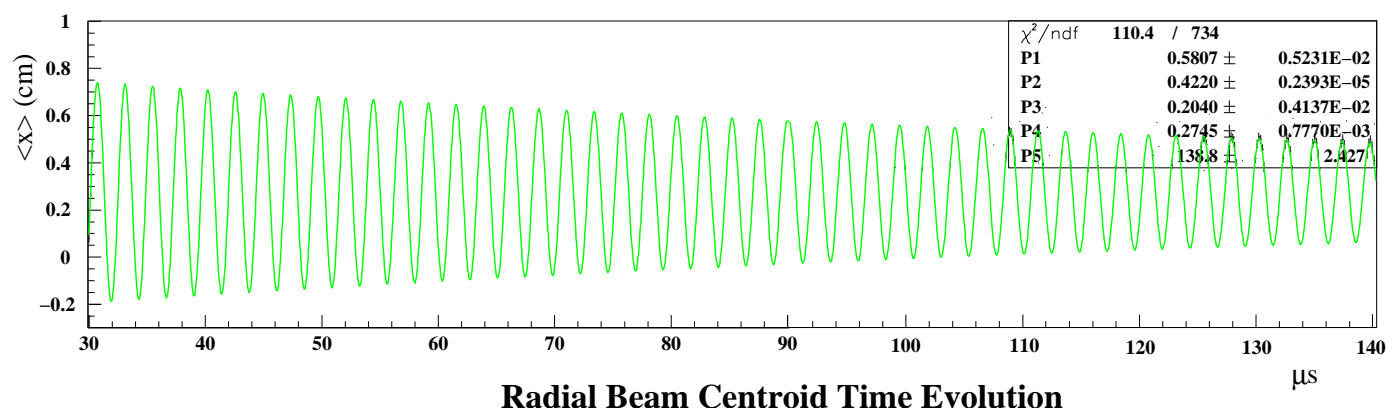

Figure 2.28: Results from g2track in "FBM" mode, with quadrupole voltages set to 21.7 $\mathrm{kV}$. The radial beam centroid from the simulation is measured mimicking what the fiber harp detectors would see, and the results are fit to a five parameter function.

in this plot: one very large resonance which seems to have a peak near $22 \mathrm{kV}$, and a smaller resonance which seems to have a peak near $26.2 \mathrm{kV}$.

We may compare the second resonance to a study done during the 2001 data run on March 8, where the number of FSD double coincidences (see Section 4.2.7) were plotted as a function of quadrupole voltage (the red triangles). These data are arbitrarily offset from zero and have rather arbitrary units, however their relative heights are valid. We clearly have a resonance which sits nearly $0.2 \mathrm{kV}$ higher than the resonance seen in the simulation. Since we know the electric fields used in the simulation do not exactly reproduce the fields in the actual experiment, it is not surprising that these resonance do not exactly overlap. Indeed, the fact that the peaks of the resonances agree to within $1 \%$ actually says much about the reliability of the simulation. Unfortunately, no comparison between the simulation to actual data for the larger resonance at the lower n-value can be made at this point, as no data were taken at these n-values.

The time spectrum and azimuthal location of muons lost in g2track is shown in Fig. 2.30. Although there are obviously too few lost muons in the distribution to be able to make a meaningful comparison to the $3 \mathrm{FC}$ time spectrum, in general the simulated time spectrum agrees with what we see in the data: most of the muons are lost before $70 \mu \mathrm{s}$, and after that the level of lost muons is more or less flat. Finally, the peaks in the azimuthal distribution of the losses in g2track seem to be about $\sim 14^{\circ}$ downstream from the collimators placed in the simulation.

\subsection{Magnetometer}

The magnetic field is measured using pulsed NMR techniques. The field inside the storage ring is mapped out a few times per week via a trolley which houses an array of 17 NMR probes. In between trolley measurements, the field is "tracked" by approximately 120 "fixed" probes, which sit above and below the vacuum chamber. Furthermore, a feedback loop consisting of a subset of the fixed probes adjusts the current in the superconducting coils to stabilize the field. Before describing the NMR probe systems used in this experiment, we first describe the fundamentals of NMR. 


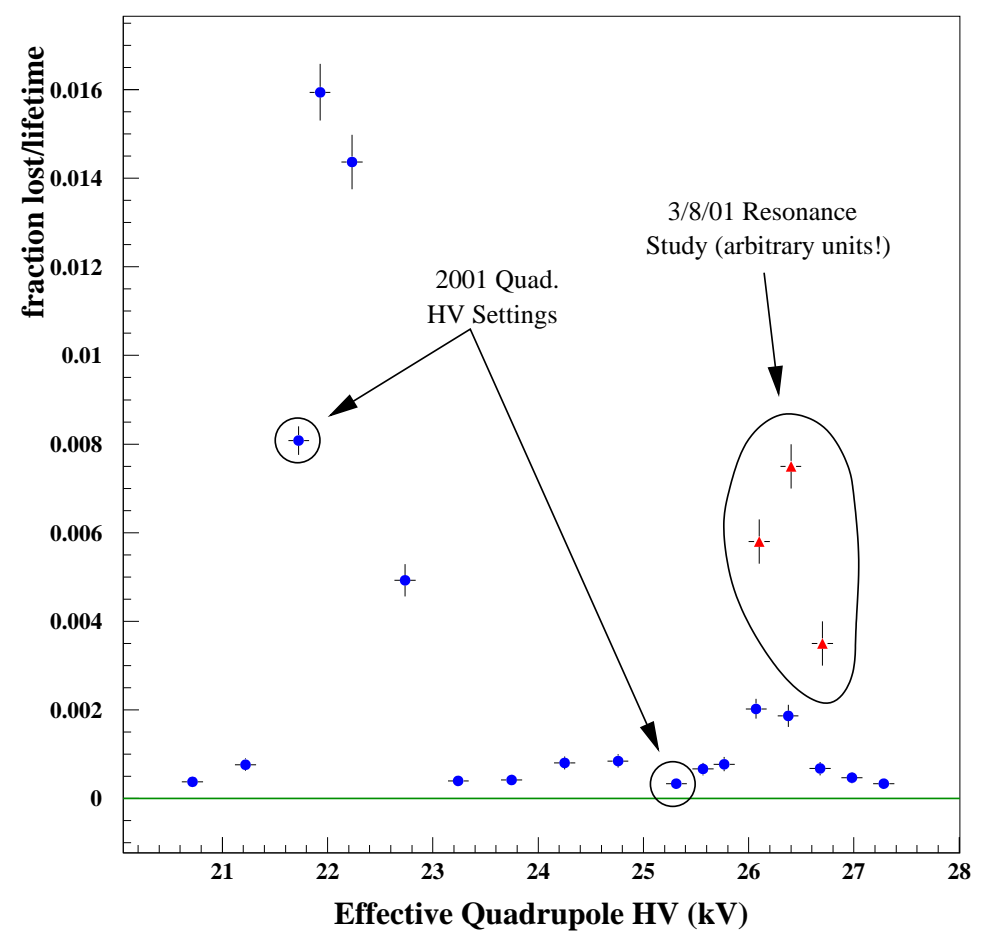

Figure 2.29: Fraction of lost muons as a function of applied quadrupole high voltage.

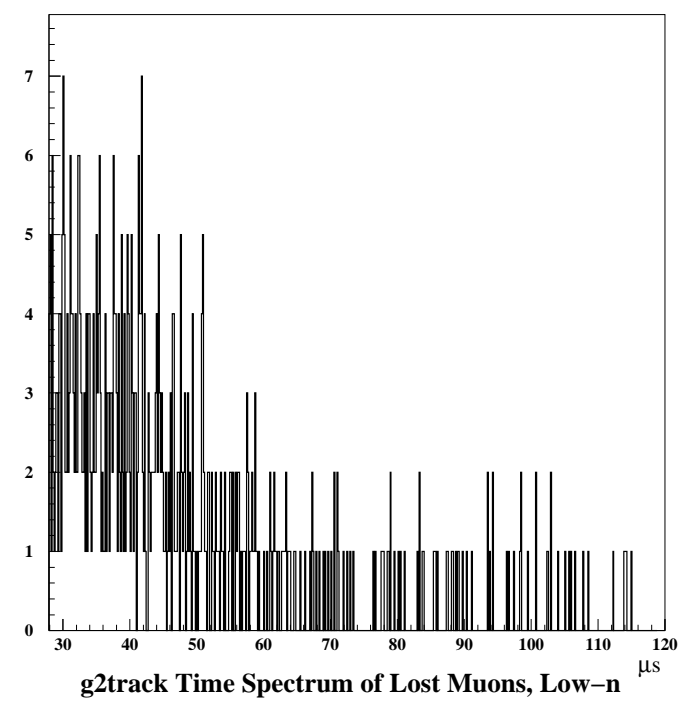

(a) Time spectrum of lost muons in g2track.

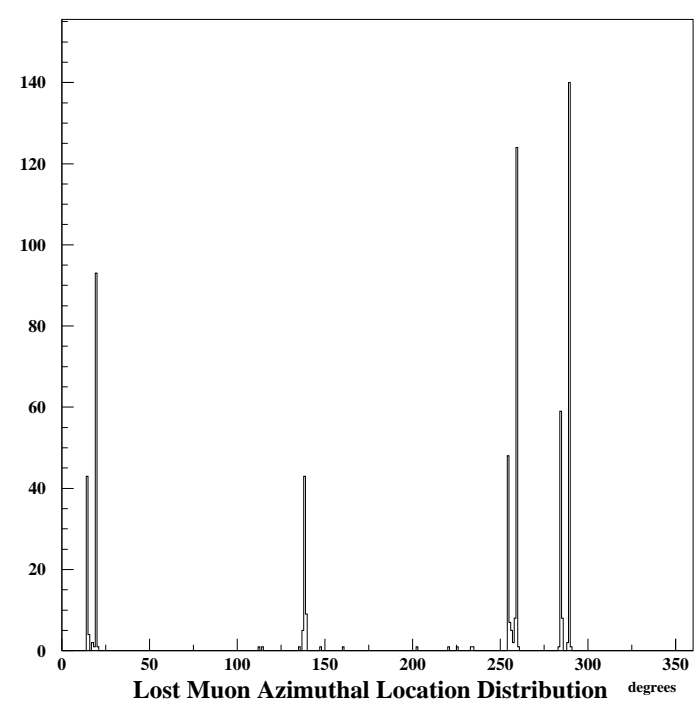

(b) Azimuthal position distribution of lost muons in g2track.

Figure 2.30: Time spectrum and azimuthal location of where muons are lost in g2track for the low-n quadrupole HV setting. 


\subsubsection{Nuclear Magnetic Resonance}

All nuclei with nuclear spin $\vec{I} \neq 0$ have a magnetic moment

$$
\vec{\mu}=\gamma_{I} \hbar \vec{I}
$$

where $\gamma_{I}$ is proportional to the gyromagnetic ratio. For a nucleus in a magnetic field $\vec{B}$, the Hamiltonian $\mathbf{H}$ is

$$
\mathbf{H}=-\gamma_{I} \hbar \vec{I} \cdot \vec{B}
$$

The proton, having spin=1/2, has only two energy eigenvalues, $E_{ \pm}=\mp \gamma_{I} \hbar B_{0} / 2$. The difference between these two energies is $\Delta E=\hbar \omega_{L}$, where $\omega_{L}=\gamma_{I} B_{0}$ is the Larmor frequency. This is the fundamental condition for magnetic resonance absorption [61]. At room temperature, the energy difference between the two eigenstates is extremely small compared to thermal energies $\left(\Delta E \sim 10^{-5} \cdot k T\right.$ for $\left.B=1.5 \mathrm{~T}\right)$. Therefore in a system containing many protons at room temperature, the populations of the two energy levels are approximately equal.

In a macroscopic sample of $N$ nuclei, the sum of the magnetic momenta, $\vec{M}$ is defined as

$$
\vec{M}=\sum_{i=1}^{N} \overrightarrow{\mu_{i}}
$$

In thermal equilibrium and in an external magnetic field, $\vec{M}_{0}$ points in the direction of the external field:

$$
\vec{M}_{0}=\frac{N \hbar^{2} \gamma_{I}^{2} I(I+1)}{3 k T} \overrightarrow{B_{0}} \equiv \chi_{0} \overrightarrow{B_{0}}
$$

where $\chi_{0}$ is the static nuclear susceptibility.

NMR measurements are made by turning $\vec{M}$ away from the direction of the magnetic field using a pulsed RF signal. A circularly polarized field $\overrightarrow{B_{1}}$ perpendicular to $\vec{M}$ is applied, which exerts a torque on $\vec{M}$ :

$$
\vec{\tau}=\vec{M} \times \vec{B}
$$

where $\vec{B}=\left(B_{1} \cos \omega t,-B_{1} \sin \omega t, B_{0}\right)$ and we have chosen our coordinate system such that the external magnetic field lies along the $z$-axis. Therefore, the equations of motion for $\vec{M}$ are

$$
\frac{d \vec{M}}{d t}=\vec{M} \times \vec{B}
$$

To explain the dynamics of the magnetization, we now choose a rotating coordinate system with axes $x^{\prime}, y^{\prime}$, and $z^{\prime}$, such that the $x-y$ plane rotates about the $z$-axis (= $z^{\prime}$-axis), as depicted in Fig. 2.31. In this frame,

$$
\left(\frac{\partial \vec{M}}{\partial t}\right)_{r o t}=\vec{M} \times \vec{B}_{e f f}
$$

where $B_{\text {eff }}=\left(B_{1}, 0, B_{0}-\omega / \gamma_{I}\right)$. As shown in Fig. 2.31, the magnetization precesses about the effective magnetic field in the rotating coordinate system. In the resonance case where $\omega$ is chosen such that $\omega=\omega_{L}=\gamma_{I} B_{0}$, the $z^{\prime}$ component of $B_{\text {eff }}$ vanishes and $\vec{M}$ rotates 


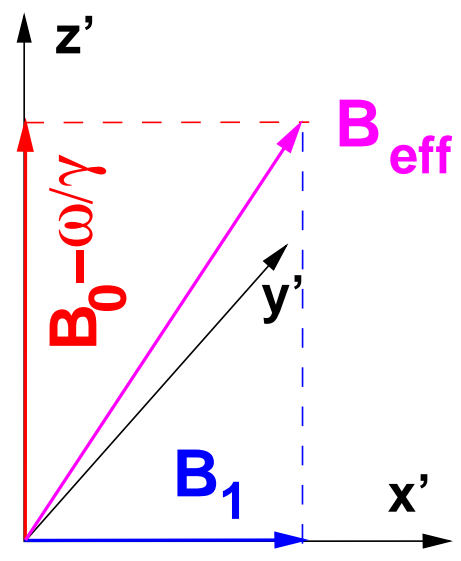

(a)

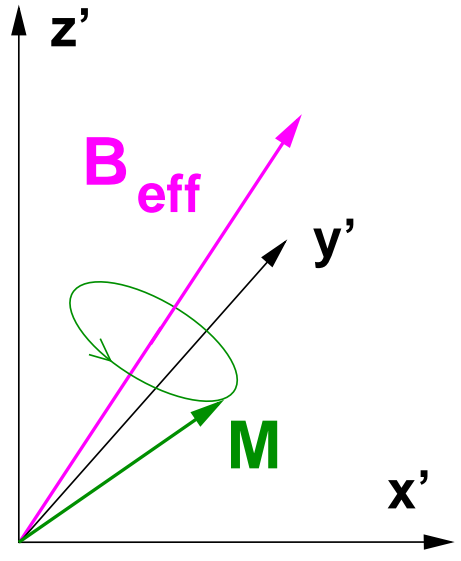

(b)

Figure 2.31: In a coordinate system with the $x-y$ plane rotating about the $z$-axis at a frequency $\omega$, the $\hat{z}$ component of the external magnetic field $B_{0}$ is reduced by $-\omega / \gamma_{I}$. The applied magnetic field $B_{1}$ is orthogonal to $B_{0}$, resulting in an effective magnetic field $B_{\text {eff }}$. A torque is exerted on the magnetization $\vec{M}$ which causes it to precess about the effective magnetic field.

about the $x^{\prime}$ axis.

If the RF signal is pulsed, $\vec{M}$ rotates about the $x^{\prime}$ axis by an angle $\alpha=\gamma_{I} B_{1} t_{p}$, where $t_{p}$ is the pulse length. Using a pulse length $t_{p}=\pi /\left(2 \omega_{L}\right), \vec{M}$ is tilted by $90^{\circ}$ such that it lies along the $y^{\prime}$ axis. In the non-rotating coordinate system, which is the lab frame, $\vec{M}$ precesses around $\overrightarrow{B_{0}}$ and emits electromagnetic radiation at the Larmor frequency, $\omega_{L}=\gamma_{I} B_{0}$.

The emitted RF signal decays in time as relaxation forces drive the magnetization $\vec{M}$ back to its thermal equilibrium value. The relaxation process is referred to as free induction decay (FID). The time constant of the FID from the proton in pure water in a very homogeneous magnetic field is of the order of a few seconds. Since a single $1 \mathrm{~ms}$ measurement of the FID signal results in a $0.05 \mathrm{ppm}$ uncertainty in the Larmor frequency, an FID time constant of several seconds is three orders of magnitude longer than is required by the proposed precision of this experiment. As a higher NMR measurement repetition rate is desirable, the time constant of the FID signal is reduced to about $30 \mathrm{~ms}$ by diluting $\mathrm{CuSO}_{4}$ in the water.

The Larmor frequency of the proton in pure water at room temperature in a magnetic field of $\sim 1.45 \mathrm{~T}$ is $\omega_{L} \simeq 2 \pi \times 61.7 \mathrm{MHz}$. The width (FWHM) of the resonance line under the same conditions is $\omega_{\text {half }} \simeq 2 \pi \times 0.1 \mathrm{~Hz}[62]$. The quality factor of the resonance line is extremely high, $\omega_{L} / \omega_{\text {half }} \simeq 6 \times 10^{8}$, which makes NMR a natural choice for making precise measurements of magnetic fields. 


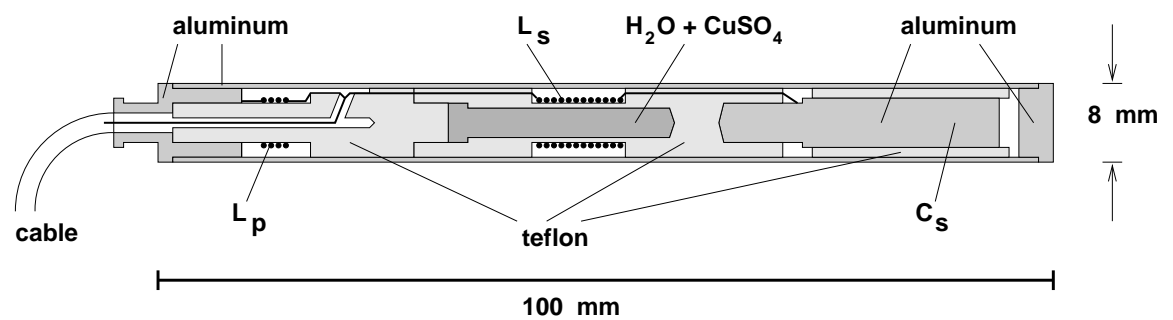

Figure 2.32: A typical NMR probe used in this experiment. The $\mathrm{H}_{2} \mathrm{O}$ and $\mathrm{CuSO}_{4}$ solution is contained in a cylindrical glass tube $15 \mathrm{~mm}$ long and $2.5 \mathrm{~mm}$ inner diameter. The cylindrical symmetry minimizes the field distortions caused by the bulk susceptibility of all parts of the probe.

\subsubsection{NMR Probes}

Fig. 2.32 shows a schematic of the trolley and fixed probes. A resonant circuit is formed by two coils with inductances $L_{s}$ and $L_{p}$ and a capacitance $C_{s}$. The arrangement of the circuit is shown in the lower-right corner of Fig. 2.33. The parallel inductor $L_{p}$ serves to match the circuit to the $50 \Omega$ line impedance. The capacitance $C_{s}$ is formed by the $\mathrm{Al}$ housing of the tube and a metal electrode; one may vary the capacitance by moving a Teflon ring in and out, allowing one to tune the resonance circuit. A pulse from a low phase noise synthesizer, stabilized by a Loran $\mathrm{C}$ receiver to $10^{-12}$ absolute accuracy [62], is sent into an amplifier which sends a several-Watt RF pulse into the trolley NMR probe for up to $10 \mu$ s, exciting the nuclear spins of the water sample.

After the RF pulse is emitted, the water sample emits a $\mu \mathrm{V}$ FID signal, which is received by the NMR probe resonant circuit. Fig. 2.33 shows a schematic of the electronics of the pulsed NMR system. The strong transmitted RF signal is decoupled from the weak FID signal through a duplexer [62]. The FID signal then passes though a low-noise preamplifier and is mixed with the synthesizer signal of a well-defined reference frequency, $f_{\text {ref }}=61.70$ $\mathrm{MHz}$. Since the reference frequency is close to the Larmor frequency $f_{L}$, the resulting beat frequency, $f_{\mathrm{FID}}=f_{L}-f_{\text {ref }}$ is in the $\mathrm{KHz}$ region. The NMR frequency is determined by counting the number of zero crossings $\left(N_{z}\right)$ of the FID signal and, simultaneously, counting the number of cycles $\left(N_{c}\right)$ of a precision clock, running at a frequency $f_{c}$. Therefore, the NMR frequency is

$$
f_{N M R}=f_{r e f}+\frac{N_{z}}{N_{c}} f_{c}
$$

The FID signal of the fixed probes is also digitized, and it is therefore possible to obtain a time spectrum (see Fig. 2.34). The frequency obtained from the Fourier analysis of the time spectrum agrees to within $0.01 \mathrm{ppm}$ of the zero-crossing measurement [63].

Three types of NMR probes are used to measure the magnetic field of the $g$ - 2 storage ring. The first type, described above and shown in Fig. 2.32 is used both in the trolley system and the fixed probe system. The second type of probe is the so-called "plunging" probe, used to calibrate the trolley probes with respect to one another. This probe, shown in Fig. 2.35, is mounted on a stand at a fixed single azimuthal position in the storage ring 


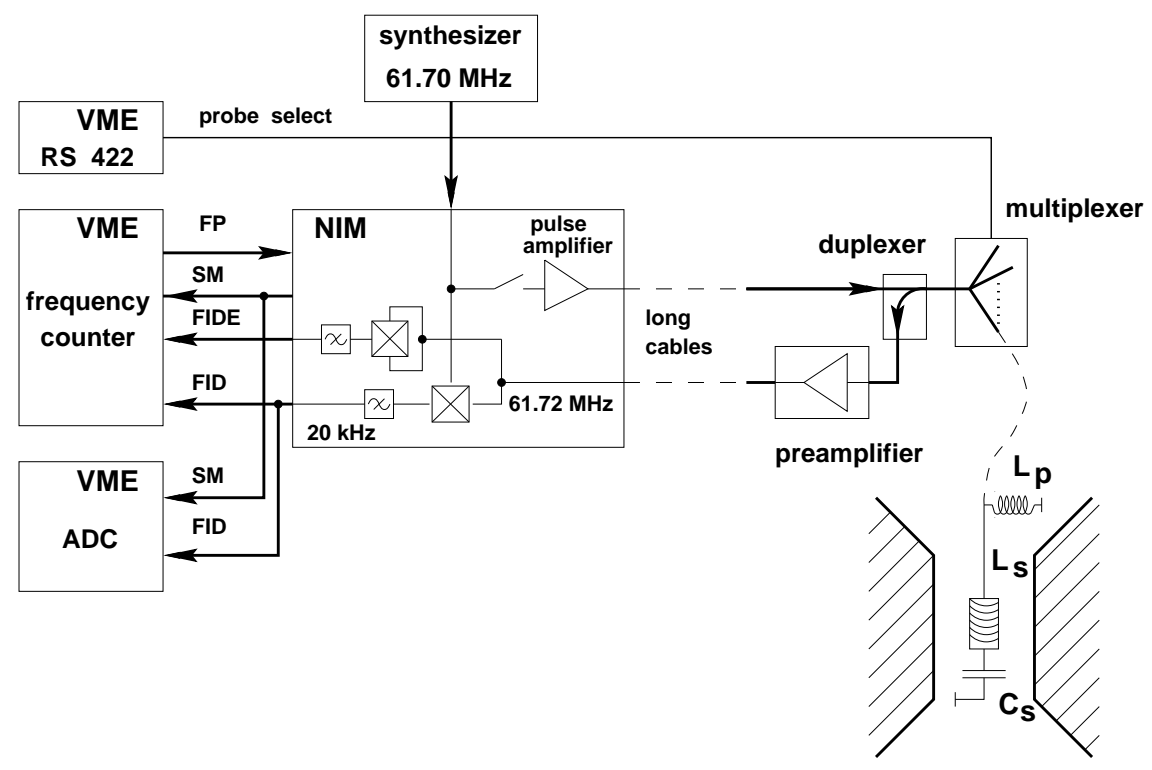

Figure 2.33: The electronics of the pulsed NMR magnetometer. The NMR probe is represented by the lower-right resonance circuit. The multiplexer allows to serve up to 20 NMR probes, and the duplexer decouples the transmitted signal from the emitted signal. The high frequency FID signal is mixed with a reference signal and the beat frequency is measured.

and may be moved into the storage aperture in both the radial and vertical directions. Note that the fundamental difference between the plunging probe and trolley/fixed probe is in the shape of the water sample. In order to determine the field in very precise locations, the water sample in the plunging probe is more localized in the spherical glass shell than the long cylinder containing the trolley/fixed probe water sample. A standard probe, shown in Fig. 2.36, provides the absolute calibration of the plunging probe and the central trolley probe. With this probe, the Larmor frequency of protons in a spherical sample of pure water was measured with a systematic uncertainty of $0.03 \mathrm{ppm}$ [63].

\subsubsection{The Trolley}

The trolley is a vacuum-tight aluminum tube $\sim 0.5 \mathrm{~m}$ long, curved to match the curvature of the storage ring. Fig. 2.37(a) is a photo of the trolley sitting inside an open vacuum chamber; Fig. 2.37(b) shows the arrangement of the 17 NMR probes used in the trolley to measure the magnetic field in different radial and vertical position inside the vacuum chamber. The trolley tube is filled with air for better heat transfer from the electronics inside to the trolley shell.

The trolley has 12 wheels which run on rails in the vacuum chamber but outside the storage aperture. Since the trolley operates in the storage ring under vacuum, frequent magnetic field measurements (once every 2-3 days) are possible and systematic errors that would arise from having to open the vacuum chamber are eliminated. When muons are 

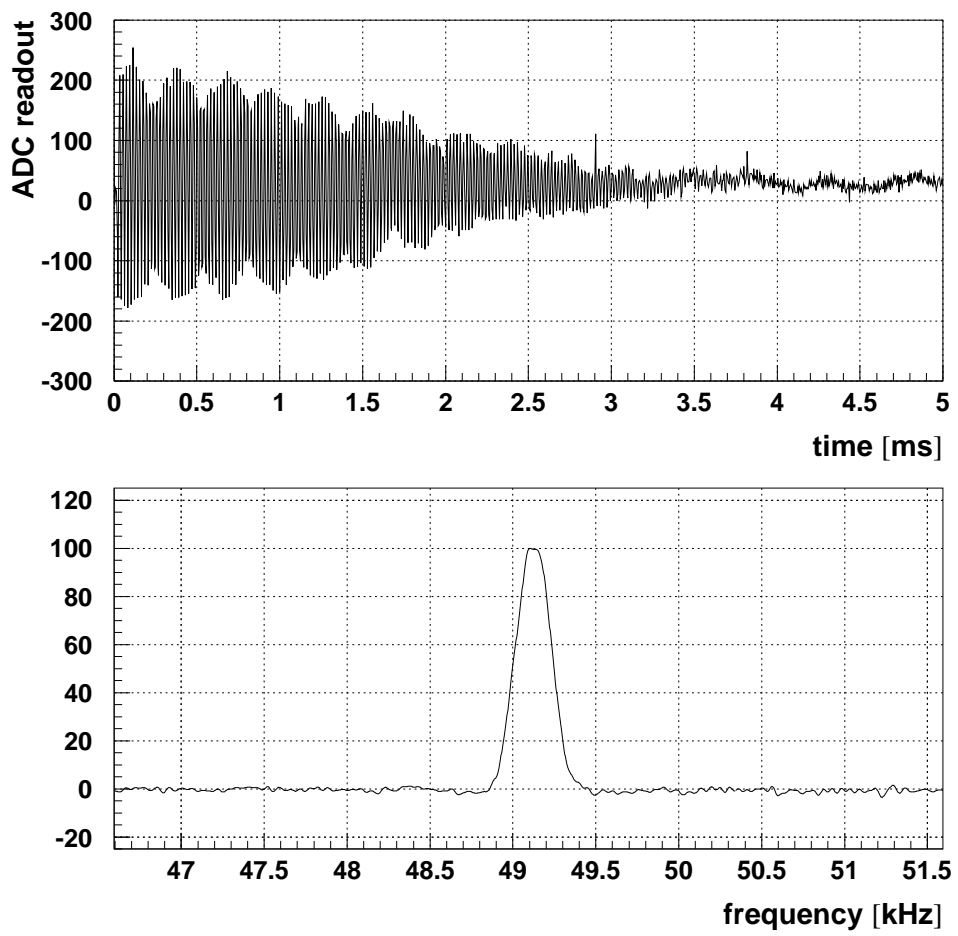

Figure 2.34: Time (top) and Fourier frequency (bottom) spectra of the FID signal obtained from a single NMR probe. The beating seen in the time spectrum is between the FID frequency and the clock, or ADC sampling frequency, and does not affect the determination of $f_{N M R}$.

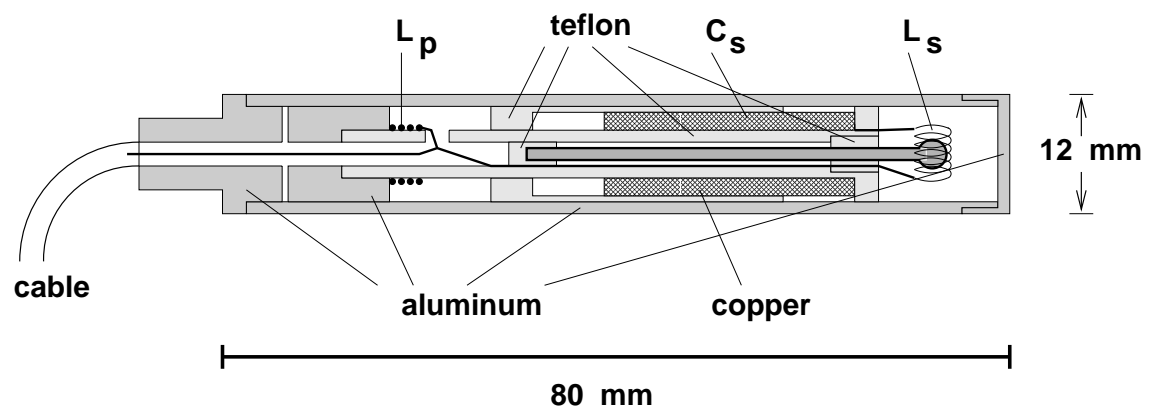

Figure 2.35: Schematic of the plunging probe used to calibrate the trolley probes. 


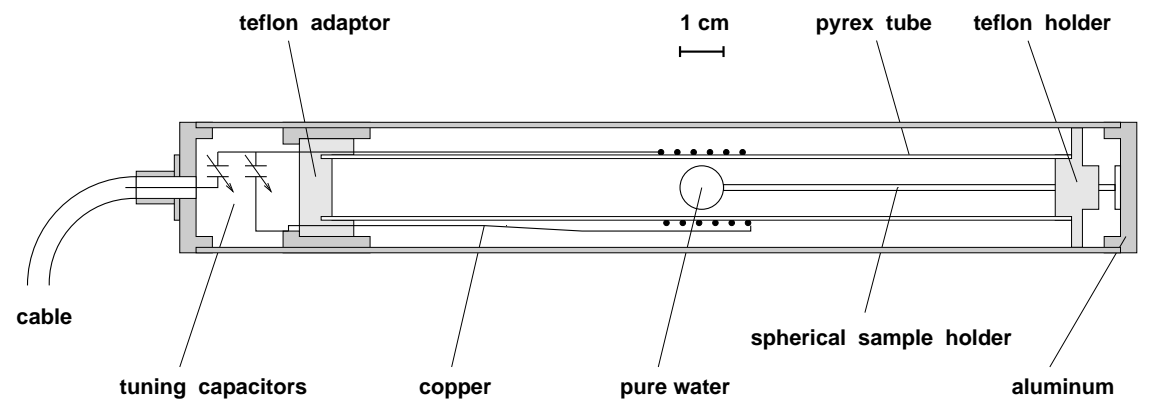

Figure 2.36: Schematic of the standard calibration probe.

injected into the ring, the trolley is stationed in a "garage" which sits outside of the storage ring aperture, but is still part of the vacuum chamber. When measuring the magnetic field, a mechanical structure in the garage pushes the trolley into position in the storage ring; two cables are then used to pull the trolley around the storage ring, one of which is used to send and receive signals to and from the electronics inside the unit. The length of the cable inside the storage ring, and hence the azimuthal position of the trolley, is determined by measuring the resistances of potentiometers attached to the drums around which the cables are wound.

The trolley NMR probes measure the absolute value of the magnetic field $|\vec{B}|$, not the direction of the field. However, the radial and longitudinal components of the field contribute less than $0.01 \mathrm{ppm}$ of the absolute value and are therefore negligible in the determination of the average B-field. Assuming $\vec{B}=\left(0, B_{y}, 0\right)$, then the 17 independent NMR probe measurements may be fit to a multipole expansion of $B_{y}$ :

$$
B_{y}(x, y)=B_{y}(r, \theta)=B_{0}+\sum_{j=1}^{4} a_{j}\left(\frac{r}{r_{0}}\right)^{j} \cos (j \theta)+\sum_{j=1}^{4} b_{j}\left(\frac{r}{r_{0}}\right)^{j} \sin (j \theta)
$$

where $x$ is the radial position inside the storage aperture, $(r, \theta)$ are polar coordinates, and $r_{0}$ is chosen to be $4.5 \mathrm{~cm}$, the radius of the storage aperture. The values of $a_{j}$ and $b_{j}$, averaged over azimuth, and the average radial and vertical position of the beam are used to determine the average magnetic field seen by the muons.

\subsubsection{Data Acquisition System}

The electronics and computers of the data acquisition (DAQ) system sit in a control room set about $50 \mathrm{~m}$ away from the storage ring. The analog signals from the NMR probes are sent to the control room through low-loss RG-213 coaxial cables. The DAQ system used for the $\omega_{p}$ measurement is a VME-based framework, shown in Fig. 2.33. A VME RS 422 module is used to select the NMR probe to be excited and read out. A VME frequency counter is used to count the number of zero crossing of the FID signal; the counter operates at $20 \mathrm{MHz}$ for the fixed probes, and $61.74 \mathrm{MHz}$ for the trolley probes. As mentioned in Section 2.5.2, additional VME ADC modules may be used to obtain actual time spectra of 


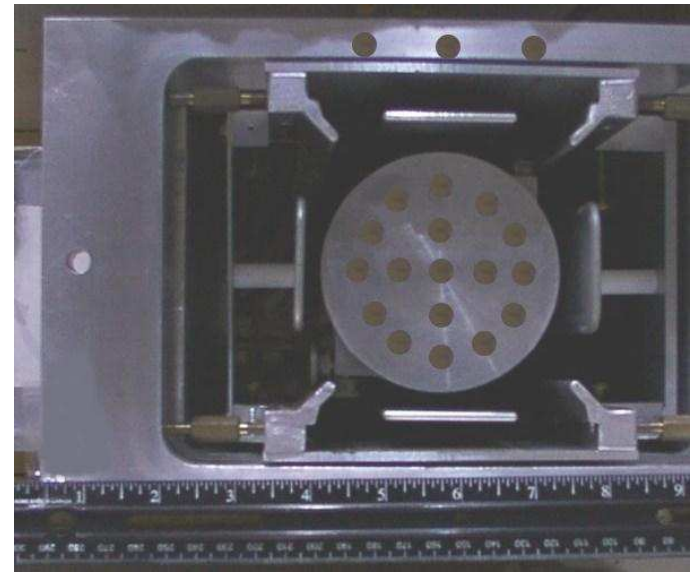

(a)

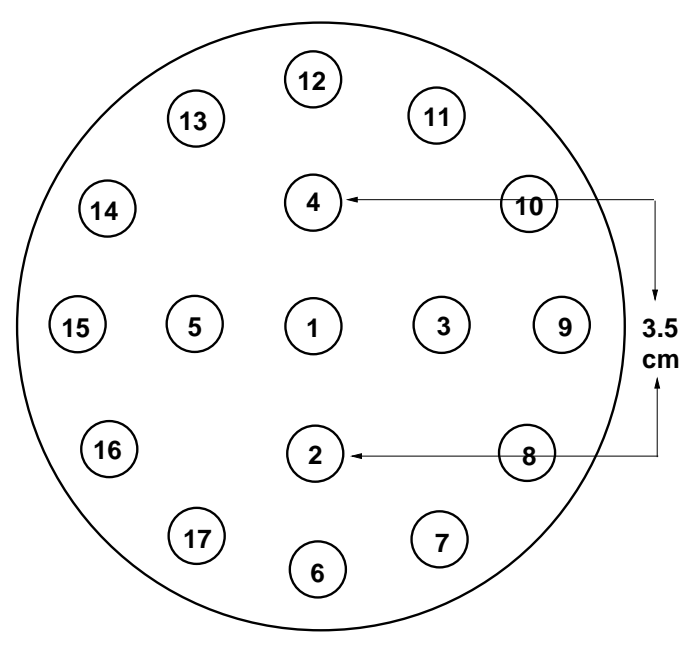

(b)

Figure 2.37: Photo of the trolley sitting on the rails inside the vacuum chamber, and a schematic of the distribution of the NMR probes inside the trolley.

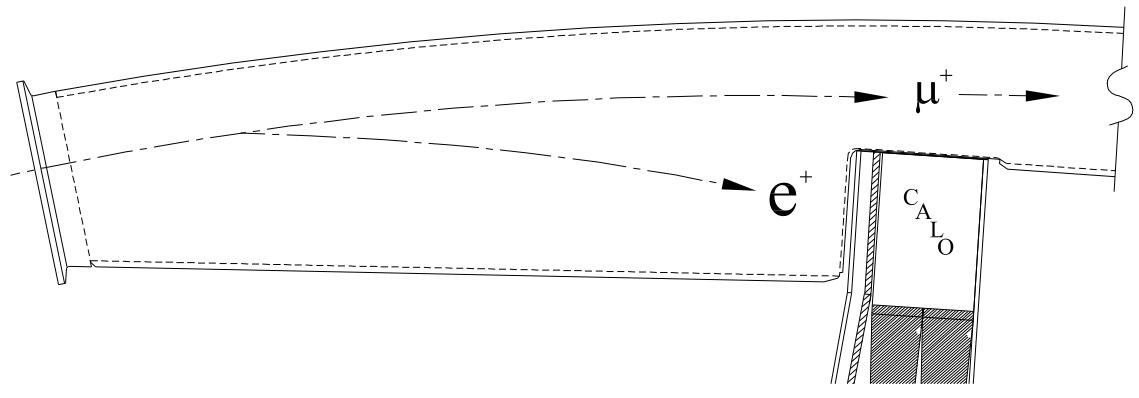

Figure 2.38: Top view of location of a single detector used in the experiment. The shape of the inner vacuum wall of the storage ring is scalloped in order to minimize scattering and energy loss of the decay electrons, improving the resolution of the detectors.

the FID signal. A PC connected to the NMR VME crate reads the data from the VME module memory buffers and saves them to disk. The PC also provides online monitoring of the magnetic field and an interface to the superconducting coil current settings.

\subsection{Muon Decay Detector System}

\subsubsection{Electron Detectors}

The measurement of the spin precession frequency is essentially a counting experiment. Twenty-four detectors, stationed symmetrically around the inside of the storage ring, de- 
termine the energy and time of the decay electrons. Fig. 2.39 shows a schematic drawing of a detector station. Each detector station consists of a lead/scintillator fiber electromagnetic calorimeter (labeled CALO). A subset of detector stations (14 in 2001) also have Front Scintillator Detectors (FSDs), and a handful (5) also have Position Sensitive Detectors (PSDs) (see below).

In order to reduce the amount of preshowering from the decay electrons as they pass through the vacuum wall of the storage ring on the way to a detector, the inner storage ring was built with a novel scalloped design (Fig. 2.38). This design results in most decay electrons exiting the vacuum chamber nearly orthogonal to the vacuum chamber. The detectors are placed very close to the outside of the vacuum chamber, in the position shown in Fig. 2.38.

To ensure a systematic shift $<0.1 \mathrm{ppm}$ in the spin precession frequency, the change in energy response must be less than $0.2 \%$ over $200 \mu \mathrm{s}$, whereas the change in the average pickoff time must be less than 20 picoseconds over the same amount of time. Therefore the precession frequency is much less sensitive to changes in the energy threshold than to changes in the average pickoff time. However, to protect the PMTs and the PMT bases (the electronics directly connected to the PMTs) from the large flash produced after each AGS injection (fill), the PMTs are gated off before and gated back on $\sim 6-20 \mu$ s after each injection. The gate-on time (the time when the PMT is switched on) depends on the detector's azimuthal position, since those detectors located closer to the injection point see more flash and may not be gated on until the neutron background drops to an acceptable level. The PMTs are required to turn on to within $\sim 0.2 \%$ of the nominal gain in less than $\sim 10 \mu \mathrm{s}$. The PMTs were chosen and their bases were carefully designed and built by the Boston University Electronics Design Facility to give the fastest rise time and reasonable gain and linearity[64].

\section{T0 Counter}

The times of all decay electrons from different fills must be properly aligned with respect to the time of injection, defined as T0. Therefore a $1 \mathrm{~mm}$ thick, $10 \mathrm{~cm}$ diameter Lucite Cerenkov counter is placed upstream of the inflector. The light output from the Lucite of this $T 0$ counter is transported into a photomultiplier tube (PMT), the output of which is recorded by a $400 \mathrm{MHz}$ waveform digitizer. The shape of the $T 0$ pulse is approximately Gaussian with a width of $27 \mathrm{~ns}$. The mean time of the T0 pulse for each fill is defined as $t=0$ for all decay electrons detected in that fill.

\section{Electromagnetic Calorimeters}

The calorimeter is made of polystyrene-based scintillating fibers embedded in a lead alloy (Pb/Sci) [65]. The fibers are $1 \mathrm{~mm}$ diameter Bicron BCF-99-49A, developed from a fluor used in BC-404 fast timing scintillator [65], oriented radially with respect to the storage ring so that most decay electrons strike transverse to the axis of the fiber.

The calorimeter has dimensions $22.5 \mathrm{~cm}$ wide $\times 14 \mathrm{~cm}$ high $\times 15 \mathrm{~cm}$ deep. The plastic-tovolume ratio was adjusted to yield a radiation length $X_{0}$ of $1.14 \mathrm{~cm}$ so that the effect of sideentering positrons is reduced while ensuring 93-96\% electromagnetic shower containment in the 1-3 GeV energy region of interest (in the center of the calorimeter). 


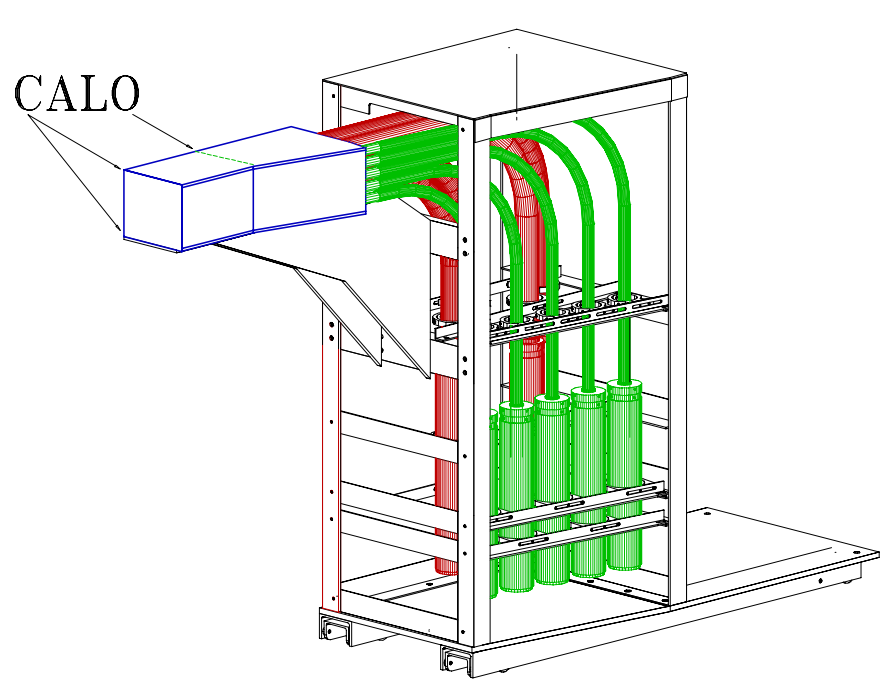

(a)

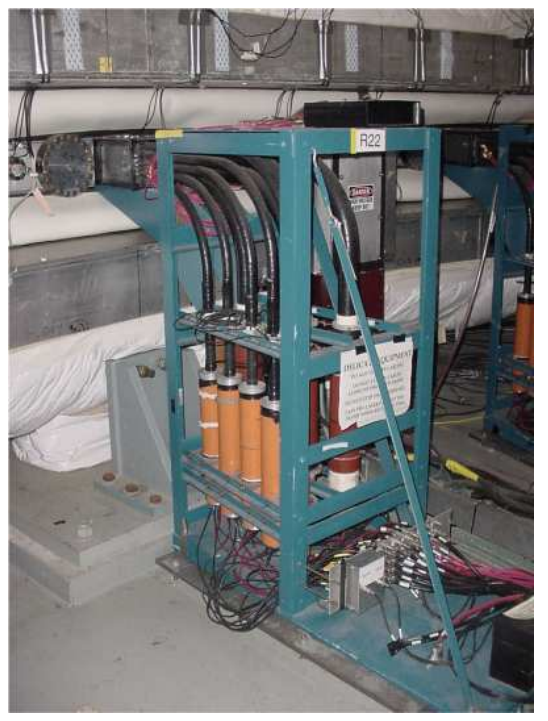

(b)

Figure 2.39: Schematic drawing and photo of one of the 24 electromagnetic calorimeters used in the experiment. Light from the electromagnetic shower in each quadrant of the detector is transported down the curved scintillator (light guides) and into individual photo-multiplier tubes.

In order to limit the amount of light each single PMT is exposed to, and in order to collect a sufficient amount of the light from the fibers and obtain uniform light collection, the calorimeter is subdivided into four quadrants. Unwrapped, tapered UV-absorbing acrylic light-guides, glued to the $\mathrm{Pb} / \mathrm{SciFi}$ block, extend away horizontally and collect the light from each quadrant. The light is then piped down a UVT acrylic rod, approximately 1 meter in length and bent $90^{\circ}$ downward. Finally, the light is collected by 2 inch Hamamatsu R1828 PMTs which have a transit time jitter of less than $240 \mathrm{ps}$ and a $1.3 \mathrm{~ns}$ rise time, allowing for fast and stable timing.

The voltages on the calorimeter PMT tubes were calibrated at the beginning of the 2001 data run using the decay electron energy spectra read out by CAMAC modules. In general the voltages were set so that the energy spectra of the top and bottom halves of the calorimeter agreed within 5-10\%. During data taking, the time response of the calorimeters were monitored with a laser calibration system. UV light emitted from a pulsed nitrogen laser is guided along quartz optical fibers to the center of the storage ring. The light is then split and guided by fibers connected to small Lucite blocks located on the large-radius side of each quadrant of the calorimeter. A very stable solid-state photodidoe was used to monitor the stability of the laser light output. A fraction of the data runs were designated "laser runs", where the laser was pulsed at pre-determined times. The pick-off time of the laser pulses by each calorimeter is then compared to the actual times the laser was pulsed. 


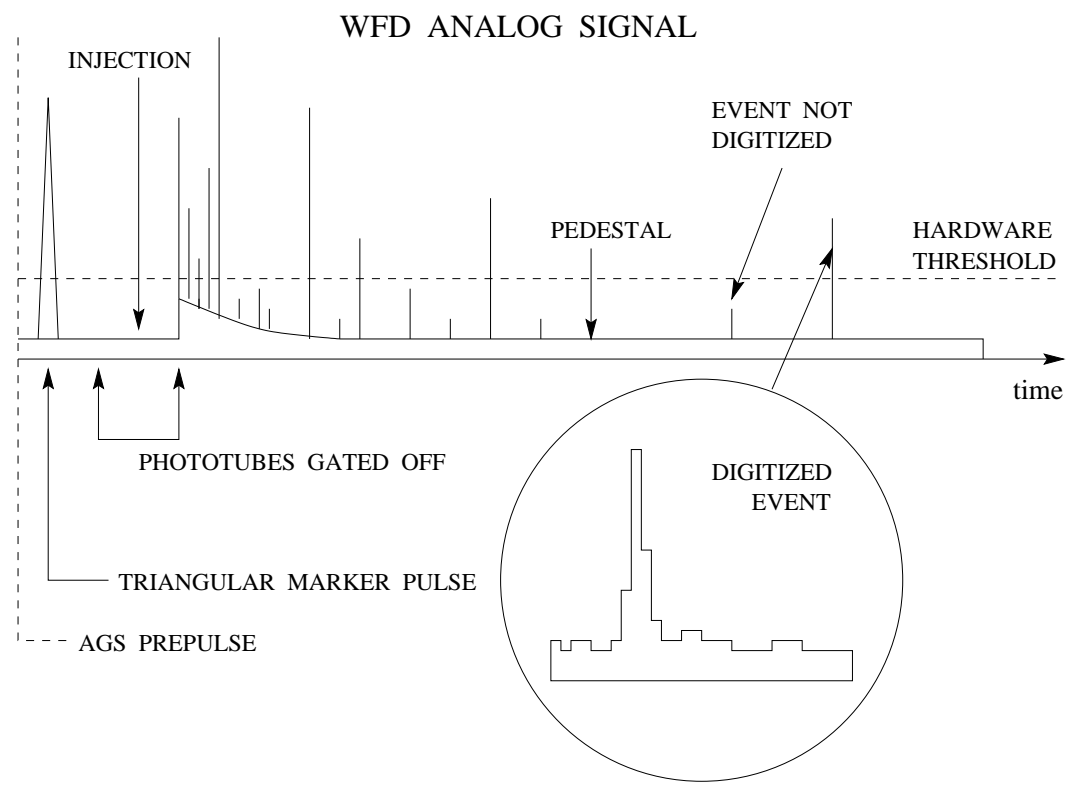

Figure 2.40: A prepulse from the AGS triggers the DAQ system to acquisition mode, and a triangular marker pulse is generated and input to each WFD module so that the two phases of the WFD may later be time-ordered properly. Only pulses above a set hardware energy threshold are written to memory and saved. Figure from [66].

\section{Front Scintillating and Position Sensitive Detectors}

To complement the electromagnetic calorimeters, just over half of the detector stations are equipped with front scintillating detectors (FSDs) and five detector stations are also equipped with position sensitive detectors (PSDs). FSDs consist of five strips of scintillator which lay horizontally across the face of the calorimeter, thus providing information on the vertical position of particles entering the front face (facing the scalloped wall) of the detector. Each strip is $2.8 \mathrm{~cm}$ wide, $23 \mathrm{~cm}$ long and $\frac{3}{8}$ inches thick, and is coupled to a gated photomultiplier through an acrylic light guide. PSDs are 2D hodoscopes made of 32 vertical tiles and 20 horizontal tiles. Each PSD tile is made of $7 \mathrm{~mm}$ wide, $8 \mathrm{~mm}$ thick scintillator coupled through a wavelength-shifting optical fiber to a multi-channel photomultiplier or hybrid photodiode. A multi-channel photomultiplier allows a large set of channels to be read out by a single device.

\subsubsection{Data Acquisition System}

As with the magnetometer, analog signals from the calorimeters and FSDs are sent to the DAQ system in the control room. The PSD signals are very small, and so to avoid noise pickup, they are discriminated with electronics near the detector station, and digital signals are sent to the control room. Analog signals of the electrostatic quadrupole voltages, muon kicker module currents, and Fiber Harp PMTs are also sent to the same DAQ system.

In the analysis of the muon decay spectrum, the times and energies of the decay electrons 
are typically determined from pulses processed by custom-built waveform digitizers (WFDs). The operation of the WFD is sketched in Fig. 2.40. These analog-digital converters (ADCs), designed and tested by the Boston University Electronics Design Facility, basically act as $400 \mathrm{MHz}$ digital oscilloscopes. The analog output of the four calorimeter PMT tubes are summed together, and then sampled by two 8-bit $200 \mathrm{MHz}$ flash ADCs (FADCs) operating at opposite phases of a $200 \mathrm{MHz}$ clock, giving an effective $400 \mathrm{MHz}$ sampling rate. The two phases are recombined by aligning the two phases of a triangle "marker pulse" which is input to the WFD $\sim 50 \mu$ s before the beam is injected into the $g$ - 2 storage ring. The marker pulse may also be used as a reference time for all WFDs.

Since the DAQ system cannot continuously process data from the electronics at the required rates, a "hardware threshold" is set such that only pulses above the threshold are digitized. The WFDs have $64 \mathrm{kB}$ of memory for each detector per phase. Half of the memory is used for time information, the other half is used for ADC samples. Time data are kept in two byte (=16 bits) segments of memory, the maximum value of which is $2^{16} \times 5 \mathrm{~ns}$ $=327 \mu$ s. In order to enforce at least one time word write within each $327 \mu$ s clock period, the WFD is put in "forced digitization" mode every $300 \mu \mathrm{s}$. Besides being used to record the calorimeter data, the WFDs are also used in single-phase $200 \mathrm{MHz}$ mode to digitize the waveforms of the voltages of the muon kickers, electrostatic quadrupoles and fiber beam monitors.

During the initial detector quadrant calibration, the individual quadrants are recorded by CAMAC [67] ADC and time-to-digital converter (TDC) modules in conjunction with the laser calibration system to determine the voltage settings of the individual calorimeter PMTs. During data taking, information from the individual calorimeter quadrants is lost by summing the four signals in the WFD. Some quadrant information is kept, however, by recording the quadrant or quadrants in which the decay electron shower takes place. Four "discriminator bit" flags are therefore attached to the time word in the WFD.

The time of the decay electron events from both the calorimeter WFD sum and the individual FSD tiles is recorded by $800 \mathrm{MHz}$ multi-channel time-to-digital converters (MTDCs). The calorimeter and scintillator pulses are converted to logic signals (discriminated), and the MTDCs record the times of the two transitions of the square pulse in 20-bit time words. The FSD hit patterns in the scintillator sticks are only recorded by the MTDCs.

The PSDs are read out with separate electronics modules operating at $50 \mathrm{MHz}$. The PSD module records both time and position information, and may be set to trigger on the calorimeter summed signal or self-triggered by a coincidence between the two detection planes [66].

Fig. 2.41 shows a block diagram of the DAQ system used for the muon spin precession measurement. The WFD, MTDC and PSD electronics are all VME [68] modules. The VME bus uses 32-bit addressing to read out the modules on a common backplane and can operate at rates of 5-20 megabytes per second $(\mathrm{MB} / \mathrm{s})$ depending on the data transfer scheme. Six $9 \mathrm{U}$ VME crates, each with its own front end CPU controller, are used in this experiment. The front end CPUs read out the memory buffers from the electronics modules after each fill of the $g$-2 storage ring and store the data on local memory. The front ends are connected to a $6 \mathrm{U}$ VME event builder crate. Since the CAMAC data is relatively small in volume, the event builder CPU reads out the CAMAC data over a VME-CAMAC interface module after each fill of the $g$-2 storage ring. However since the VME data is quite large in volume, 


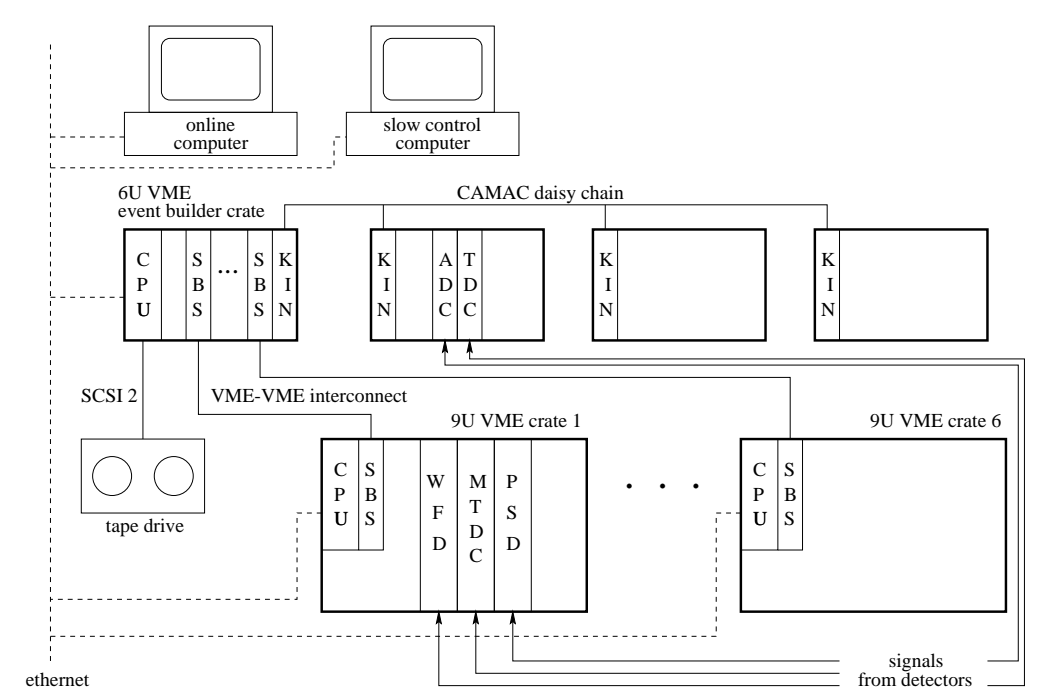

Figure 2.41: Block diagram of the DAQ system. Figure from [66].

the data in the front end memory is only read out by the event builder CPU between AGS cycles, at which point the event builder CPU writes all VME and CAMAC data to tape via a SCSI-2 connection.

The VxWorks [69] operating system (OS) is used on all front end and event builder CPUs; a computer using the Linux [70] OS runs the UNIDAQ [71] data acquisition software, and connects the user to the VME CPUs via an ethernet connection. A fraction (typically 5$10 \%$ ) of the VME data is copied to the Linux-based computer which displays the appropriate time and energy distributions in real time. Data collection is broken up into short periods called runs, which are numbered for ease of reference. A run typically contains about $5-25 \times 10^{6}$ decay electrons with energies $>1.8 \mathrm{GeV}$ and decay times $>32 \mu$ s.

A time stamp from a common $100 \mathrm{~Hz}$ timer is recorded by both the $\omega_{a}$ and $\omega_{p}$ DAQ systems so that the data may be correlated in the offline analysis. Furthermore, both DAQ systems use a $10 \mathrm{MHz}$ reference frequency provided by a Stanford Research Systems (SRS) FS700 module locked to the Loran-C standard radio frequency. The SRS FS700 module is stable at the $10^{-10}$ level, and the Loran-C is stable to $10^{-12}$. However, since the final value of $a_{\mu}$ involves the ratio $\omega_{a} / \omega_{p}$, any shifts in clock frequencies tend to cancel out.

\subsubsection{Data Production}

Two computer programs convert the raw WFD, MTDC and PSD data to easily readable and accessible formats. The two data productions are based on very different frameworks; the G2OFF production is based on Fortran and the HBOOK [72] and CERNLIB [73] libraries, and the g2Too production is based on $\mathrm{C}++$ and the ROOT [74] framework. Although the two productions are implemented using very different frameworks, the algorithms used for determining the energies and times of the decay electron pulses in the calorimeters are very similar; they really only differ in their implementation. Because in this dissertation only data from the g2off production were used to determine $a_{\mu}$, some of its more relevant 
features are described below.

\section{Energy and Time Extraction Algorithm}

In order to extract the time and energy of the decay electron pulses in the calorimeter, the time-ordered WFD data for each fill and each detector are broken into segments, or "islands". Pulses are identified as local maxima, and the length of the island is defined by a maximum time distance between consecutive pulses, typically $29 \mathrm{~ns}$. An average pulse shape for each detector is constructed [75] using $\sim 10 \times 10^{3}$ decay electrons $200 \mu$ s after injection, at which time most islands have only one pulse and effects such as PMT gain, flash and pileup are reduced to a negligible level. In the construction of the average pulse shape, it is assumed that the pulse shape does not depend on the amplitude (energy) of the pulse. This is of course true on average as long as the detector response is independent of time.

The WFD ADC samples of all pulses are then fit to an average pulse shape with a constant background (the pedestal) to obtain an energy and time. The amplitude of the pulse scales linearly with the energy (or area under the curve) of the pulse; the energy may therefore be obtained in arbitrary units of ADC counts from either the area or the amplitude of the peak of the pulse. The ADC counts are later normalized to energy units of "GeV" using the end points of the decay electron energy spectrum (see Section 4.2.1). Detailed simulation studies showed [76] that the pulse-finding algorithm employed by the G2OFF production has an effective low-energy threshold of $\sim 250 \pm 33 \mathrm{MeV}$, and a small $(3 \mathrm{MeV})$ systematic shift in the fitted amplitude of the pulses. The shift is inconsequential since it is later normalized away; however there is a small time-dependence of the fitted amplitude of pulses of about 0.05 ADC counts, or $<0.05 \%$ for pulses above $1.5 \mathrm{GeV}$, from $\sim 32-100 \mu$ s after injection. As will be shown in Section 4.2.5, this time-dependence of the reconstructed energy of decay electron pulses is two to four times smaller than other effects resulting in "energy-scale changes", and we may safely ignore this effect.

The time associated to each pulse, the "pick-off" time, is the fitted time of the pulse peak. The uncertainty of the pick-off time is typically less than $100 \mathrm{ps}$ [76] with a systematic shift of $\sim 35 \mathrm{ps}$, depending on the energy. More importantly, the early-to-late change in the average pick-off time in the fill is less than 2 ps.

\section{Data Storage Format}

The data are saved in the format of ntuples, data structures containing large amounts of related information, accessible using either the interactive Physics Analysis Workstation (PAW) program or the HBOOK Fortran library routines. A simplified diagram of the structure of the ntuples used in the G2OFF production is shown in Fig. 2.42. Appendix A contains a full list of the 84 variables contained in the G2OFF ntuple. For each data run and for each detector, data are saved in a time-ordered array of "events", or AGS fills. Electrostatic quadrupole voltages, muon kicker voltages, T0 pulse parameters, and many other parameters are kept in a general data "block". Separate data blocks of all time-ordered calorimeter WFD, FSD and (not shown in Fig. 2.42) PSD events are created for each event. Note that not all FSD events correspond to the same indexed WFD event; this is because in the g2off production, FSD events are saved when there is a WFD event within a given time window. 


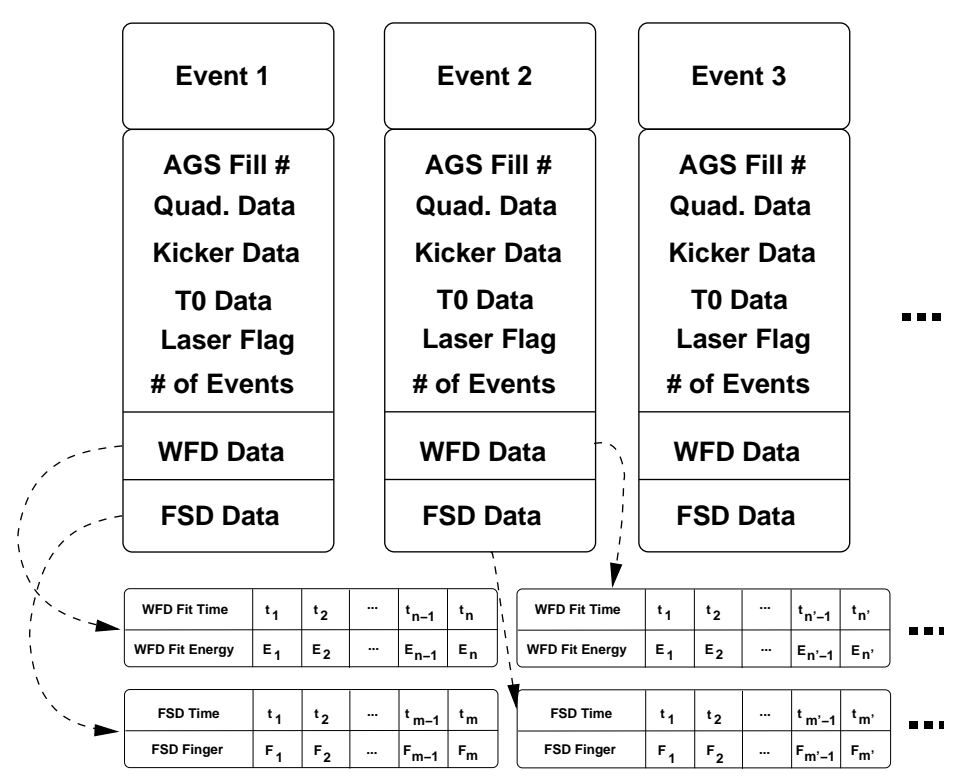

Figure 2.42: Simplified block diagram of the ntuple data structure created by the G2OFF production for each detector and data run. Much more information is actually kept than is shown here.

Since there are many low-energy pulses that are not seen by the WFD (below the hardware threshold), several FSD pulses could fall within the WFD time window. It is up to the analyzer to match WFD events to the FSD events.

\subsection{Previous Results of E821}

Experiment 821 at BNL has taken data over several months every year from 1997 until 2001. With the exception of 2001, all "runs" measured the magnetic moment of the positive muon; the polarity of all magnets were reversed for the 2001 measurement.

The first "run", in 1997, used the same pion injection scheme as the CERN III experiment. This was a commissioning run, where the various subsystems of the experiment were tested. The value obtained for the anomaly agreed very well with the previous CERN experiments, with a similar precision of $13 \mathrm{ppm}$ [77]:

$$
a_{\mu^{+}}(1997)=11659250(150) \times 10^{-10}
$$

The following year the muon kicker was commissioned and data were taken using the muon injection scheme. The benefits of muon injection were immediately obvious: the flash in the detectors was greatly reduced and the number of muons stored in the ring per AGS fill increased by an order of magnitude. The 1998 data run was cut short when a leak in the water cooling unit of the Q1 magnet near the AGS V-line production target caused it to overheat and fail. Still, in the few weeks that data were taken with the muon kicker, a 5 
ppm measurement of $a_{\mu^{+}}$was made [78]:

$$
a_{\mu^{+}}(1998)=11659191(59) \times 10^{-10}
$$

The decay positron time spectrum was fit to the five-parameter function

$$
N(t)=N_{0} e^{-t / \tau}\left(1+A \cos \left(\omega_{a} t+\phi\right)\right)
$$

and the total systematic uncertainty on $a_{\mu^{+}}$was estimated to be $1 \mathrm{ppm}$.

The 1999 run saw a 20-fold increase in the amount of data collected over the 1998 run, resulting in a 1.3 ppm measurement [79]:

$$
a_{\mu^{+}}(1999)=11659202(14)(6) \times 10^{-10}
$$

With the huge increase in the amount of data, various backgrounds in the decay positron time spectrum, such as pileup, coherent betatron oscillations and muon losses, were no longer lost in the noise of large statistical fluctuations. Indeed, the fit $\chi^{2} /$ d.o.f. of the five-parameter function proved insufficient to fully describe the data at the $\sim 100 \sigma$ level. Therefore, the fit function and/or the data itself was modified to account for these effects, resulting in an acceptable fit $\chi^{2} /$ d.o.f. and a total systematic uncertainty of $0.3 \mathrm{ppm}[79]$.

In 2000, another four-fold increase in the amount of data collected over the 1999 run was achieved by increasing the flux of the AGS, and a $0.7 \mathrm{ppm}$ measurement was made [80]:

$$
a_{\mu^{+}}(2000)=11659204(7)(5) \times 10^{-10}
$$

Several improvements were made that reduced certain systematics; a new superconducting inflector magnet improved the homogeneity of the magnetic field and thus reduced the overall systematic uncertainty on the measurement of $\omega_{p}$. AGS backgrounds, extraneous particles from other bunches in the AGS ring, were reduced by about a factor of 10 using a "sweeper" magnet in the straight, pion-decay-channel beamline. The sweeper magnet, which was also used in the 2001 data run, is a traditional ferrite kicker with an RC time constant of $700 \mu \mathrm{s}$. It is turned off just before each AGS fill of the $g$ - 2 storage ring, after which it produces a maximum field of about $50 \mathrm{mT}$ in $15 \mu \mathrm{s}$. The sweeper magnetic field provides a $\sim 5$ mrad kick to charged particles, effectively sweeping the beamline clean. However, there was an increased rate and high level of flash in the detectors from attempts to inject and store more muons in the ring, which forced the use of high hardware energy thresholds in the WFDs. This resulted in rather late fit start times of the data, at $\sim 50 \mu \mathrm{s}$ after injection, a significant loss of data. Furthermore, as with the previous years, one "sees" backgrounds more clearly with smaller statistical fluctuations: both the asymmetry, $A$, and phase, $\phi$, in the positron decay time spectrum are modulated at the CBO frequency, $f_{C B O}$. The $\omega_{a}$ analysis was complicated by the fact that the effective weak-focusing field index value of $n \simeq 0.137$ used in the 2000 data run resulted in a value of $\omega_{C B O}$ very close to twice the $g$ - 2 frequency, $\omega_{a}$. If not properly dealt with, the interference frequency, $\omega_{C B O}-\omega_{a}$, from the asymmetry and phase CBO modulations produce time-dependent shifts in the fitted value of $\omega_{a}$ of up to $4 \mathrm{ppm}$. The results of many careful studies showed a systematic uncertainty in $\omega_{a}$ due to $\mathrm{CBO}$ of $\sim 0.2 \mathrm{ppm}$, with an overall systematic uncertainty of 0.3 


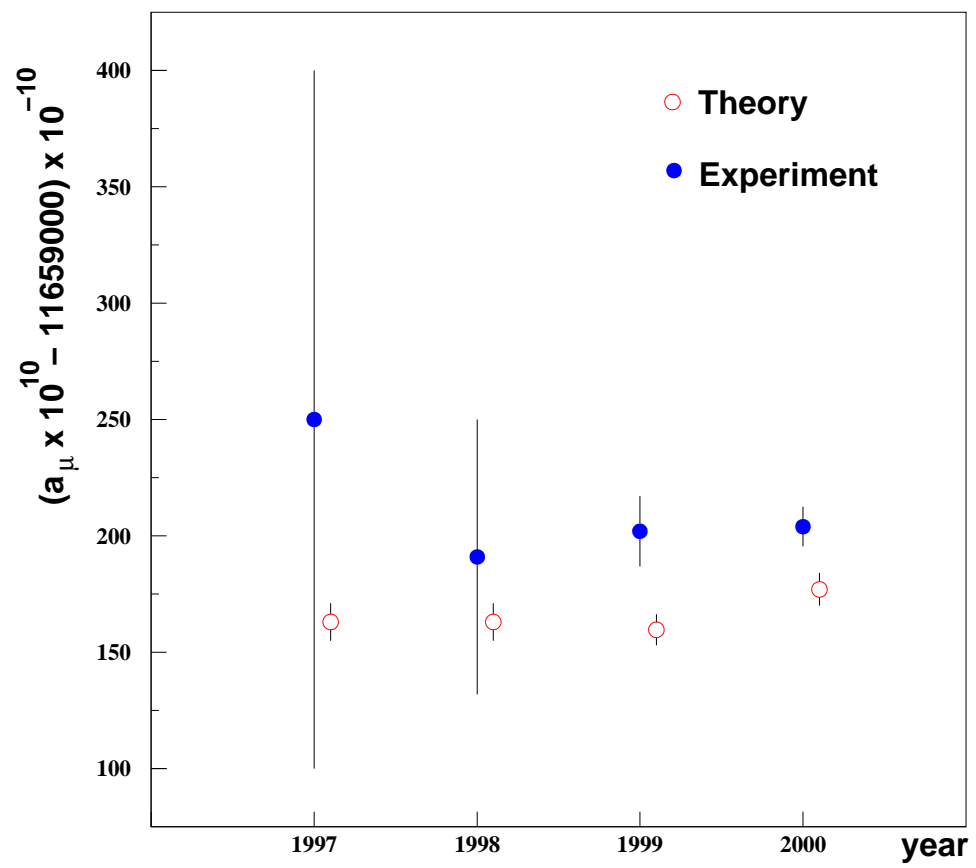

Figure 2.43: Brookhaven E821 measurements of $a_{\mu}$ as a function of time. For comparison, the theoretical values of $a_{\mu}$ as reported in each year's publication is shown in red.

$\operatorname{ppm}[80]$.

The 2001 run conditions were set to resolve and/or possibly study further the problems found from the analysis of the 2000 data. The rates in the detectors and the hardware energy thresholds were lowered to allow earlier fit start times. Data were collected at two different $n$-values, $n \simeq 0.122$ and $n \simeq 0.142$, which correspond to CBO frequencies that are below and above the second harmonic of the $g$-2 frequency by $\sim 30 \mathrm{kHz}$, respectively.

Fig. 2.43 shows the evolution of the experimental and theoretical values of $a_{\mu}$ since the 1997 run. The E821 Collaboration published results[77, 78, 79, 80] of the analysis of each data run, and the theoretical values shown in Fig. 2.43 are those reported by the collaboration at the time each year's experimental result was published. The jump in the theory value between 1999 and 2000 was the result of the re-analysis of the hadronic lightby-light contribution discussed in Section 1.4.2.

The agreement of the measured values of $a_{\mu}$ from year to year is remarkable. Furthermore, over a span of less than four years, and in less than 16 months of actual data taking, the statistical uncertainty was reduced by an order of magnitude and the systematic uncertainty reduced by more than a factor of two. Each year has presented a challenge in the analysis of the data, and as will be discussed, 2001 is no exception. 


\section{Chapter 3}

\section{Magnetic Field Measurement}

The magnetic field was measured, in terms of $\omega_{p}$, by the trolley 20 times during the 2001 run, and changes in the field between each trolley measurement were monitored using the fixed probes. Two independent analyses of the magnetometer data were done by E. Sichtermann and H. Deng. Two trolley probes, 4 and 8, did not function properly and were therefore discarded by both analyses. The analyses differed in the treatment of the calibration data, in the evaluation of the average field measured by the trolley, and in the selection of the fixed probes used to interpolate the field between trolley measurement. These points will be discussed further in Section 3.6.

The measurement of the magnetic field is limited by systematic uncertainties. Some of the more obvious uncertainties arise from the calibration of the trolley probes, from the trolley measurements, from the fixed-probe interpolation of the field drifts and from uncertainties in the muon distribution.

\subsection{Standard Probe Calibration}

As discussed in Section 2.5.2, the standard probe measures the Larmor frequency of the proton in a spherical water sample, $\omega_{p_{\mathrm{w}}}$. Since we wish to use the Larmor frequency of the free proton, $\omega_{p_{\mathrm{f}}}$, a correction to the measured value of $\omega_{p_{\mathrm{w}}}$ must be applied to account for the temperature-dependent diamagnetic shielding of the water molecule, $\sigma_{\mathrm{H}_{2} \mathrm{O}}(T)$ :

$$
\omega_{p_{\mathrm{w}}}=\omega_{p_{\mathrm{f}}}\left(1-\sigma_{\mathrm{H}_{2} \mathrm{O}}(T)\right)
$$

The value of the shielding constant at $34.7^{\circ} \mathrm{C}$ has been measured $[81,82]: \sigma_{\mathrm{H}_{2} \mathrm{O}}\left(34.7^{\circ} \mathrm{C}\right)=$ $25.790(14) \times 10^{-6}$. The temperature dependence of $\sigma_{\mathrm{H}_{2} \mathrm{O}}$ in the temperature range of $5-45^{\circ} \mathrm{C}$ was independently measured [83], $\sigma_{\mathrm{H}_{2} \mathrm{O}}(T)=10.36(30) \times 10^{-9} /{ }^{\circ} \mathrm{C}$. The spherical shape of the water sample, the use of pure $\mathrm{H}_{2} \mathrm{O}$ and the careful design and choice of materials used in the probe reduce to a negligible level systematic effects resulting from the bulk diamagnetism of the water sample, paramagnetic impurities in the water sample, and paramagnetic and diamagnetic impurities in the probe structure. Extensive tests of the standard probe determined an overall systematic uncertainty of $0.034 \mathrm{ppm}$ in the absolute value of the free proton Larmor frequency [62]. Uncertainties in the temperature of the standard probe increase the uncertainty of the absolute calibration from $0.034 \mathrm{ppm}$ to 0.05 


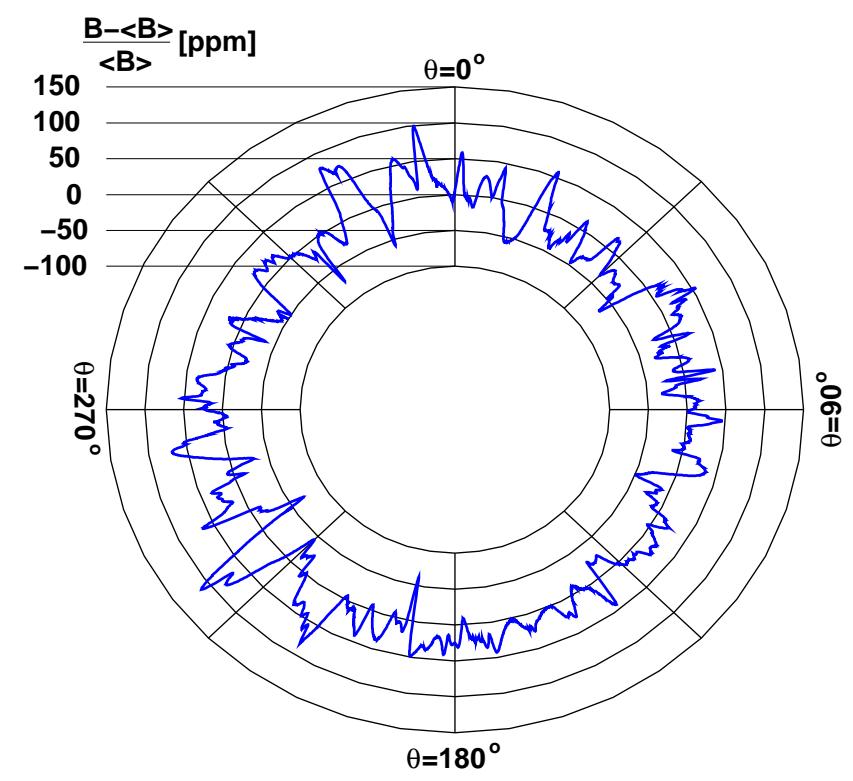

Figure 3.1: Relative fluctuations of the center trolley probe measurement of the magnetic field around the storage ring. Figure from H. Deng.

ppm.

\subsection{Trolley Probe Calibration and Measurements}

The trolley probes are calibrated relative to the plunging probe by measuring the field with each at the same azimuthal location in the storage ring. Both the plunging probe and the center trolley probe are then calibrated absolutely to the standard probe. We therefore expect systematic errors to arise from both the absolute and relative calibrations.

To minimize systematic uncertainties from positioning the trolley probe during the calibration measurements, currents in the surface correction coils were adjusted to minimize the magnetic field gradients at the position of the plunging probe. Six comparisons of the trolley probes were made using the plunging probe during the 2001 run, and from the average RMS scatter of these comparisons, an uncertainty of $0.07 \mathrm{ppm}$ is assigned to the relative calibration of the trolley probes.

The standard probe was used to calibrate the center trolley probes and the plunging probe before and after the run. Six measurements were made, the average of which is the final calibration used in the $\omega_{p}$ analysis. The uncertainty of the absolute calibration of the trolley probes is taken as the RMS of the distribution of the measurements, 0.05 ppm. Adding the relative and absolute calibration uncertainties in quadrature, the total systematic error on $\omega_{p}$ from the calibration with respect to the standard probe is $0.09 \mathrm{ppm}$.

Fig. 3.1 shows the relative homogeneity of the magnetic field around the ring, measured by the center trolley probe. The fluctuations of 50-100 ppm are very large compared to the uncertainty in each single measurement of $\sim 0.09 \mathrm{ppm}$. Therefore, uncertainties in the 


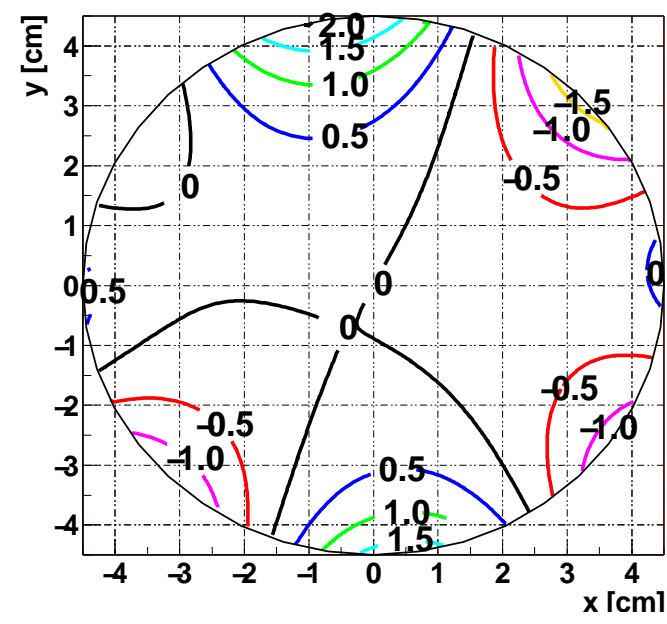

\author{
Multipoles (ppm) \\ \begin{tabular}{rrr} 
normal & skew \\
Quad & -0.29 & 0.15 \\
\hline Sext & -0.71 & -0.48
\end{tabular} \\ Octu $\quad 0.06 \quad 0.01$ \\ Decu $\quad 1.08 \quad 0.39$
}

Figure 3.2: Contour plot of the dipole magnetic field, averaged over azimuth, in the storage aperture. All values are relative differences with respect to the field in the center of the aperture. The contour lines are 0.5 ppm wide. Figure from H. Deng[84].

trolley probe measurement are dominated by the uncertainty in the azimuthal position of the trolley as it is pulled through the ring.

In 2001, two methods were used to determine the trolley's position in the ring. The first, employed in previous years' analyses, uses two potentiometers attached to the drums which pull the trolley cables. The readings from the potentiometers have uncertainties up to $20 \mathrm{~cm}$. However, as the trolley passes a fixed probe, a jump in the reading of the fixed probe frequency is seen. By correlating the passage of the trolley to jumps in the fixed probe readings, the uncertainties in the position of the trolley are reduced to $2 \mathrm{~cm}$. A new method uses an encoding system which directly measures the rotation of the drums. Again, the position measurements are corrected using the fluctuations in the fixed probe readings. The two methods of determining the azimuthal position of the trolley agree to within $\sim 0.1^{\circ}$, and the average difference between the measured dipole field using the two methods in $\sim 0.02 \mathrm{ppm}$. Since the potentiometer method has slightly worse resolution than the encoding system, the latter is used to determine the azimuthal position of the trolley. The uncertainty of the trolley probe position during the measurements is $1 \mathrm{~cm}$, resulting in a systematic uncertainty of $0.05 \mathrm{ppm}$ in the final value of $\omega_{p}$. Fig. 3.2 shows a contour plot of the dipole magnetic field, averaged over azimuth.

\title{
3.3 Fixed-Probe Tracking of the Field Between Trolley Mea- surements
}

Between the trolley measurements, fixed probes are used to monitor and to help maintain the average magnetic field to within $0.1 \mathrm{ppm}$. Limits on changes in the fixed probe frequency are set by comparing the average field determined by the trolley to the average field inferred by the fixed probes. In 2001, there was typically about a $25 \mathrm{ppm}$ difference between the 
average dipole field measured by the trolley and the fixed probes. Because of hysteresis, the difference shifted after the currents for the main magnet and inflector were ramped down and back up, so care must be taken to compare only those points when the currents in the magnets had not just been ramped down. With this constraint, 14 changes in the difference between trolley and fixed-probe average fields were available to monitor changes in the fixed probe readings. The systematic uncertainty on $\omega_{p}$ from changes in the fixed-probe tracking of the field is calculated from the RMS of the distribution: $0.11 \mathrm{ppm} / \sqrt{2}=0.08 \mathrm{ppm}$.

The fixed probes are located uniformly along the tops and bottoms of the pole pieces of the magnet. There is a small gap at each junction of the pole pieces which affects the local field. Therefore, those probes that are located near the azimuthal edges are given less weight than those probes that are located at the azimuthal center of the poles. The differences between trolley and fixed-probe fields discussed above depend on the fixed-probe weights. The weights are therefore determined by minimizing the RMS of the distribution of changes in these differences. The optimal normalized weights found in 2001 were 0.683 for the fixed probes at the azimuthal centers of the poles and 0.317 for the fixed probes at the azimuthal edges of the poles.

\subsection{Averaging over Muon Distribution}

The determination of $a_{\mu}$ requires a measurement of the magnetic field in the muon storage ring, averaged over the muon density distribution, $\rho_{\mu}(\vec{r})$ :

$$
\left\langle\left|\overrightarrow{\omega_{p}}\right|\right\rangle=\left\langle\omega_{p_{y}}\right\rangle=\frac{\int \rho_{\mu}(\vec{r}) \omega_{p_{y}}(\vec{r}) d^{3} r}{\int \rho_{\mu}(\vec{r}) d^{3} r}
$$

However, because the muon lifetime is much longer than the radial and vertical betatron oscillation periods, and because the magnetic field is so uniform, it is sufficient to use

$$
\left\langle\omega_{p_{y}}\right\rangle=\left\langle\omega_{p_{y}}\right\rangle(\bar{x}, \bar{y})
$$

where the magnetic field has been averaged over azimuth, and $\bar{x}$ and $\bar{y}$ are the average radial and vertical positions of the stored muon beam. The uncertainties in the average radial and vertical positions of the stored muons are taken as $\pm 1 \mathrm{~mm}$ (from the fast rotation analyses) and $\pm 2 \mathrm{~mm}$ (from FSD studies) respectively. Using the amplitudes of the quadrupole moments of the field at the storage aperture limits, the systematic uncertainty on $\left\langle\omega_{p}\right\rangle$ from uncertainties on $\bar{x}$ and $\bar{y}$ is $0.03 \mathrm{ppm}$ [85]. g2track simulations which incorporate a full 3-dimensional map of the magnetic field determined from trolley measurements have confirmed that the difference between Eqs. 3.2 and 3.3 is smaller than the systematic error on $\left\langle\omega_{p}\right\rangle$ arising from uncertainties on $\bar{x}$ and $\bar{y}[86]$.

\subsection{Other Systematic Effects}

Other, more subtle, systematic uncertainties are related to higher multipoles, kicker eddy currents, changes in the trolley power supply voltage and temperature effects on the trolley probes and electronics. These effects are lumped together as a single "others" systematic 
uncertainty. Although each effect results in a relatively small uncertainty in the measured field, together they contribute a level of uncertainty comparable to the effects discussed above.

A multipole expansion involves a sum over an infinite number of multipoles. However, since the field is quite uniform inside the storage ring, only the first five multipoles of the expansion for $B_{y}$ are determined from fits to the trolley data. Measurements of the field outside the storage aperture using the the shimming trolley (which is physically larger in radius than the trolley) confirmed that the size of the coefficients of the multipole terms higher than the decapole moment are smaller than the the lower order terms of the expansion. The missing higher multipole systematic error is estimated to be $<0.05 \mathrm{ppm}$.

The muon kicker produces eddy currents primarily in the kicker plates themselves, but also in the the vacuum chamber walls. These eddy currents create a time-dependent remnant magnetic field (RMF) after the kicker pulse; if large enough, the RMF could influence the average magnetic field seen by the stored muons. The RMF produced by the kicker was measured using the Faraday Effect technique described in [53]. For a $95 \mathrm{kV}$ kick, the systematic effect of the resulting RMF on the average magnetic field is measured to be less than $0.1 \mathrm{ppm}$ at $\sim 20 \mu$ s after injection.

The trolley probes and electronics are sensitive to changes in both the trolley power supply and temperature changes. The average linear dependence of the measured field as a function of operating voltage was determined in the $9.4-10.4 \mathrm{~V}$ range to be $0.38 \mathrm{ppm} / \mathrm{V}$. Since the trolley operated in the much smaller range of 9.85-9.90 V, the uncertainty from trolley power supply fluctuations is $0.02 \mathrm{ppm}$ [85]. Similarly, the dependence of each of the trolley probes on the ambient temperature was measured to be $0.027 \mathrm{ppm} /{ }^{\circ} \mathrm{C}[85]$. The uncertainty on the average ambient temperature during all trolley runs is less than $1^{\circ} \mathrm{C}$. An upper limit of the systematic uncertainty from temperature fluctuations is $0.03 \mathrm{ppm}$.

Adding these uncertainties together in quadrature, a conservative estimate of the uncertainty on $\omega_{p}$ from "other" effects is set to $0.10 \mathrm{ppm}$.

\subsection{Final Value of $\omega_{p}$}

The two $\omega_{p}$ analyses were cross-checked to verify computations and internal consistency. One way in which the analyses differ is how significant field drifts during some of the calibration measurements were handled. These drifts were observed by fixed probes $\sim 10^{\circ}$ away from the plunging probe stand at the bottom of the vacuum chamber, but not observed by the fixed probes above the vacuum chamber at the same location. Although the drift is likely due to changes in surface coil currents, the true cause was not identified. Regardless of the cause, the effect of these drifts on the calibration measurements must be taken into account properly.

For the trolley probe calibrations, Sichtermann rejected an entire sequence of calibration measurements and Deng rejected only those measurements corresponding to times when large drifts were observed. In the case of the calibration of the center trolley probe with respect to the plunging probe, Sichtermann averaged trolley measurements before and after the trolley was moved into position, whereas Deng used the measurement with the smallest observed field drift. There were three plunging probe measurements common to 


\begin{tabular}{|l|c|}
\hline \multicolumn{2}{|c|}{$\omega_{p}$ Systematic Error Table } \\
\hline \hline Source of Error & Size $(\mathbf{p p m})$ \\
\hline Absolute calibration & 0.05 \\
\hline Calibration of trolley probes & 0.09 \\
\hline Trolley measurements of $B_{0}$ & 0.05 \\
\hline Interpolation with fixed probes & 0.07 \\
\hline Uncertainty from muon distribution & 0.03 \\
\hline Others & 0.10 \\
\hline \hline Total & 0.17 \\
\hline
\end{tabular}

Table 3.1: Systematic error table for the $\omega_{p}$ measurement.

both analyses, and the RMS width of the distribution of the differences of the measurements is $\sim 0.03 \mathrm{ppm}$, within the trolley and plunging probe calibration systematic uncertainty.

Although all trolley measurements were used by both analyses, the technique used to calculate the dipole field integral differed. Deng used a grid of $\sim 9000$ points and corrected for non-linearities of the trolley probe position readout using the perturbation of the fixed probes as the trolley passes. Sichtermann used the measured field values and determined the azimuthal position of the trolley from the optical encoders. Using a common relative calibration of the trolley probes, the average fields determined by both analyzers agreed to within $0.04 \mathrm{ppm}$, again within the systematic uncertainty of the trolley measurement of the average field.

Finally, differences exist in the way the analyses treated the fixed probes. Over half of the fixed NMR probes were found to be unreliable and were therefore not used to monitor the field. Sichtermann used 175 fixed probes, whereas Deng used 129 probes. Nearly all of the probes in Deng's selection are part of Sichtermann's. Both analyses find the same optimal values of the weights assigned to the fixed probes near the pole centers and near the pole edges, $68 \%$ and $32 \%$ respectively. Since fixed probe measurements are perturbed by the passage of the trolley, the fixed probe measurement must be interpolated from data points before and after the trolley passes. Deng interpolates using measurements at fixed time intervals before and after the peak in the perturbation and Sichtermann interpolates using measurements at a fixed distance away from each fixed probe. A comparison of the calculated average dipole fields of the two analyses as a function of run number shows that the average fields agree to within $0.02 \mathrm{ppm}$, within the quoted systematic uncertainty.

The final values of both analyses agree to within $0.02 \mathrm{ppm}$, and the systematic uncertainties determined by both analyses also agree at the same level. Table 3.1 lists the systematic errors discussed above, and the total systematic error is taken from the quadratic sum of all entries in the table. Applying the $\sim 26 \mathrm{ppm}$ correction for the diamagnetic shielding of the water molecule discussed in 3.1, the final value of the free proton resonance frequency is $\omega_{p} /(2 \pi)=61791400(11) \mathrm{Hz}$. 


\section{Chapter 4}

\section{Spin Precession Frequency Measurement}

\subsection{Data Selection}

During the 2001 run, data were collected using two different electrostatic quadrupole highvoltage settings, $21.7 \mathrm{kV}$ and $25.3 \mathrm{kV}$, corresponding to effective $n$-values of $n \simeq 0.122$ and $n \simeq 0.142$, respectively. For this analysis the data were divided into two subsets, the "low-n" (for $n=0.122$ ) and the "high-n" $(n=0.142)$.

The data selection involved a series of quality and stability requirements, or "cuts". As discussed in more detail below, some requirements are checked on a run-by-run basis, others are checked injection-by-injection (fill-by-fill). Individual decay electrons are only cut based on software lower and upper energy thresholds.

To allow room for the traceback chambers, the vacuum wall just upstream is not scalloped. As a result, many electrons pass through the wall at a glancing angle and have a long path length through the aluminum. Furthermore, some electrons pass through the thick flange of the vacuum window upstream of the first traceback chamber[87]. The passage of the decay electrons through so much material results in very poor energy resolution and a distorted energy spectrum in detector 20. Therefore, no data from this detector are used in the analysis of $\omega_{a}$, a $4 \%$ loss in the number of counts.

\subsubsection{Run Selection}

The "golden", high-quality data runs used in this analysis were selected by C. Polly, X. Huang, E. Sichtermann and others. Runs were selected based on experimental stability (eg: only runs after the detectors were calibrated and the electrostatic quadrupoles were "stable" were selected), accessibility/readability (some runs were simply lost or unreadable), and data content. Details of the run selection may be found in [88].

In 2001, data runs were numbered from 9000 to 11384. From them a total of 978 runs were selected, beginning at run number 9423 and ending at run 11384. 606 of the runs were of the low-n data set. This corresponds to, roughly, $1.7 \times 10^{9}$ high-energy decay electrons integrated between 32 and $675 \mu$ s for the low-n data set, and $1.2 \times 10^{9}$ for the high-n data 
set. With an average asymmetry of $\sim 0.38,2.9$ billion decay electrons corresponds to a relative statistical uncertainty of $\sim 0.77 \mathrm{ppm}$ for $\omega_{a}$.

An additional "silver+bronze" $(\mathrm{S}+\mathrm{B})$ set of runs was also analyzed. The $\mathrm{S}+\mathrm{B}$ set includes data taken under non-ideal, but still acceptable, run conditions, such as low kicker and low scraping voltages runs. In addition, 13 golden runs which were missing from the initial g2off production were reprocessed. All but four of the $\mathrm{S}+\mathrm{B}$ runs were taken with the low-n quadrupole high voltage settings. The "S+B" list consists of 54 runs, which contain a total of approximately $160 \times 10^{6}$ high-energy decay electrons after $32 \mu \mathrm{s}$. Note, however, that systematic studies were not done separately for this data set, which is only $1 / 10$ the size of the larger low-n data set.

\subsubsection{Fill Selection}

Quality-control was further enforced with fill cuts. We require that the electrostatic quadrupoles be on for at least $700 \mu$ s after injection and that there be no sign of quadrupole sparking. In the G2OFF ntuple, quadrupole voltage information is kept in the IQUAD, QEARLY, QMID and QLATE variables. IQUAD is an integer number giving the length, in $\mu \mathrm{s}$, of the quadrupole pulse. QEARLY is an array of measurements of the voltages of the quadrupoles in arbitrary ADC counts at the time of injection. Similarly, QMID is an array of measurements of the voltages $\sim 45 \mu$ s after injection, and QLATE is an array of measurements of the voltages 30 $\mu \mathrm{s}$ before the quadrupole voltage drops to $80 \%$ of the QMID value. The QEARLY, QMID and QLATE variables are averaged over 12 samples $(1$ sample $/ \mu \mathrm{s})$ from the times stated. To see if the quadrupoles held charge and did not spark during a fill, we verify that the variables QEARLY, QMID, and QLATE are not zero and that differences between these quadrupole readings are not "out of range". The range is determined from the distributions of (QMID-EARLY) and (QLATE-QMID) values. By requiring (QMID-QEARLY) $<40$ ADC counts and (QLATE-QMID) $<15$ ADC counts, fills where the quadrupoles sparked or completely failed are effectively removed.

We also look for fills with missing (or "bad") $T 0$ pulses, fills where the laser was fired and fills with a very small $(<15)$ number of pulses per calorimeter. Fig. 4.1(a) shows the percent of "good" fills vs. run number. The low percentage of good fills early on in the data-taking period is the result of the T0 cuts (see Fig. 4.1(b)). During these runs, the voltage on the $T 0$ counter was set so low that often the $T 0$ pulse was below threshold, and disappeared from the data stream. Although the low $T 0$ counter voltage was eventually noticed and fixed, approximately $15 \%$ of all fills failed the T0 cut. However in terms of useful data, only $3.7 \%$ of the total number of pulses above the hardware threshold were lost.

Late in the analysis, Q. Peng discovered a large number of "narrow pulses" in the g2of $f$ data. Narrowness of a pulse is defined as

$$
n=\frac{a\left(k_{\max }-1\right)+a\left(k_{\max }+1\right)}{a\left(k_{\max }\right)}
$$

where $a(k)=I S A M(k)-P E D, I S A M$ is an array of 8 ADC samples of the pulse in the WFD, $k=1,8, k_{\max }$ is the element in the array with the largest value (the peak of the pulse) and $P E D$ is the minimum value in $I S A M$. Narrow pulses therefore have a small value of $n$, 


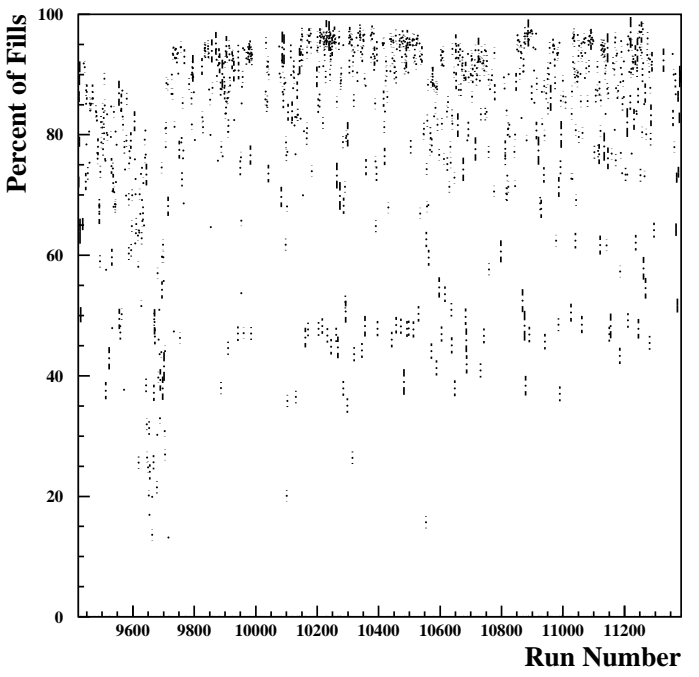

(a) Percent of "good" fills vs. run number.
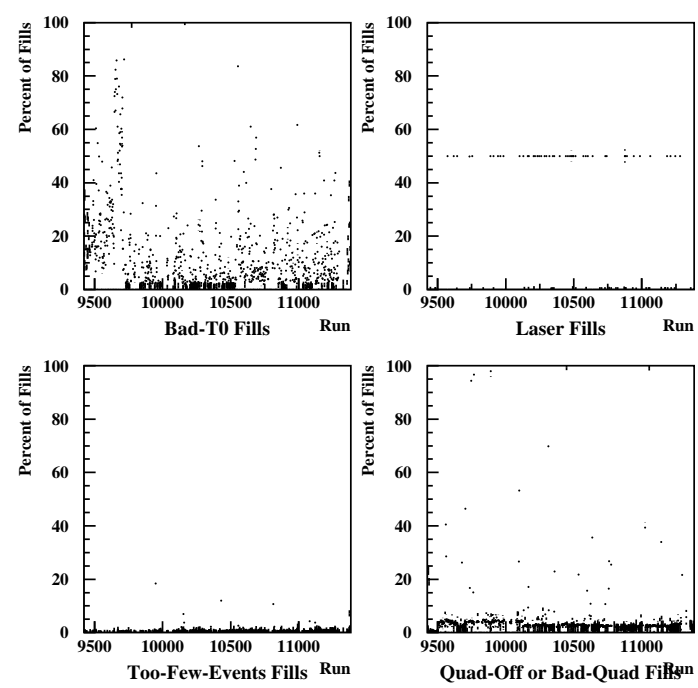

(b) Percent of the different kinds of "bad" fills vs. run number.

Figure 4.1: Fill selection as a function of run number.

and "normal" pulses have values of $n$ ranging from 1-2. Peng also succeeded in discovering two types of narrow pulses, one with "one peak", and the other with "two peaks". The two peak narrow pulses have been identified as pulses for which the two phases of the WFD were not properly aligned. Noise on the baseline before the marker pulse confused the WFD phase-alignment algorithm. Since the times and energies of all the pulses in such misaligned fills are wrong, they must not be included in the final data set. Peng has also shown that nearly all narrow pulses have a FITCHI $2^{1}$ greater than 20 , whereas "good" pulses have a FITCHI2 less than 18. Therefore, fills for which the average FITCHI2 is greater than 20 are thrown out.

\subsection{Construction of Decay Electron Time Spectra}

\subsubsection{Energy Calibration}

Since the energy of a decay electron pulse is extracted from the WFD, the height of the pulse is in arbitrary units of ADC counts. It is useful, although not necessary, to determine a conversion between units of ADC counts and the more physical units of GeV. Since the maximum energy a decay electron can have is $3.1 \mathrm{GeV}$, we use the endpoint of the decay energy spectrum to determine the conversion factor between ADC counts and GeV and track long time-scale changes in each detector's response. The endpoint of the energy spectrum

\footnotetext{
${ }^{1}$ The FITCHI2 is an ntuple variable quantifying the quality of the fit to the pulse, similar to an unnormalized $\chi^{2}$. One cannot expect the average FITCHI2 to have a value of 1 , and indeed the distribution of FITCHI2 over all pulses has a mean value close to 3 and a very long tail, out to 18-20.
} 

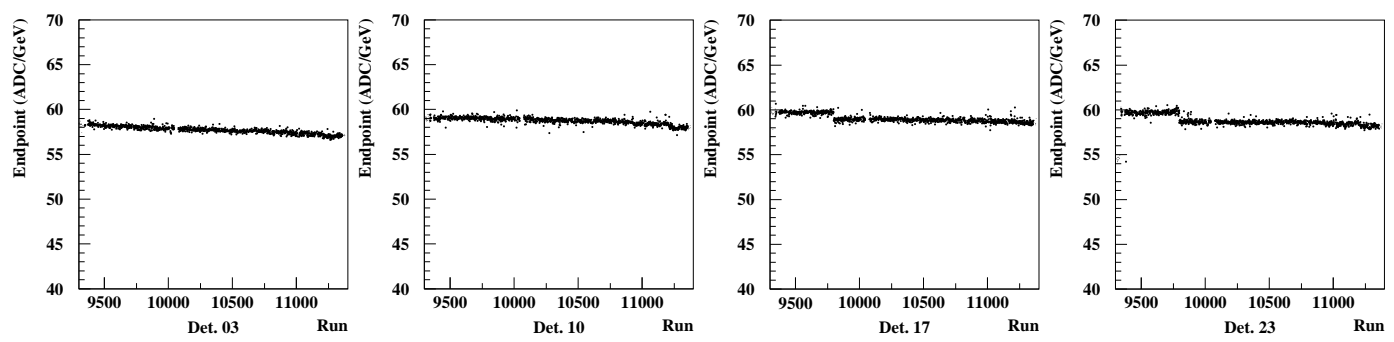

Figure 4.2: Energy calibration on a run-by-run basis for detectors 3, 10, 17 and 23. The slow change over time in the energy calibration could be the result of temperature changes and/or degradation of the detectors and electronics. The small, sudden jumps in normalization are the result of changes in voltages on the detector PMTs.

is determined by fitting a line to the region between $\sim 60-90 \%$ of the maximum value, and extrapolating to zero counts. The point where it crosses the axis (the "endpoint") is then assumed to be $3.1 \mathrm{GeV}$. The endpoints for the G2OFF data production were determined by X. Huang. Fig. 4.2 shows the endpoints for four individual detectors $(3,10,17$ and 24$)$ on a run-by-run basis. We see that over time the energies of the pulses drop by nearly $2 \%$, and in some cases there are sudden jumps in the $\mathrm{ADC}$ to $\mathrm{GeV}$ conversion. Temperature changes most likely cause the long-term drift in the conversion factor, although degradation of the detectors and electronics is also possible. The sudden jumps in the conversion factor have been traced to changes made in the applied calorimeter PMT voltages.

\subsubsection{Determination of Lower Energy Threshold}

Since $N$ and $A$ are both functions of the lower energy threshold, we may minimize the statistical uncertainty of the measurement of $\omega_{a}$ by choosing our lower energy threshold such that $\left(N A^{2}\right)$ is maximized. Therefore, time spectra were constructed for each detector and each run with various lower energy thresholds. The time spectra were then summed over runs and fit to the five-parameter function:

$$
N_{5}(t)=N_{0} e^{-t / \tau}\left(1+A \cos \left(\omega_{a} t+\phi\right)\right)
$$

starting with $t>180 \mu$ s after injection. At these late start times, many background effects such as pileup, muon losses, $\mathrm{CBO}$ and gain changes are minimized.

From these fits, we obtain $A\left(E_{t}\right)$ and $N\left(E_{t}\right)$ (and therefore $N A^{2}\left(E_{t}\right)$ ), where $E_{t}$ is the energy threshold in units of GeV. The plots in Fig. 4.3 show the dependence of $A, N$ and $N A^{2}$ on energy threshold for detector 1. A $7^{\text {th }}$ order polynomial is fit to the histogram of $N A^{2}$ vs. $E_{t}$, and the root of the derivative of the fitted polynomial is determined using the CERNLIB function RZERO. Fig. 4.4 shows the threshold energies where $N A^{2}$ is a maximum for each detector, the same thresholds that are used to fill the time-spectrum histograms. To eliminate as much pileup as possible, an upper energy cut of $3.2 \mathrm{GeV}$ is applied to all detectors when filling the time spectra. 


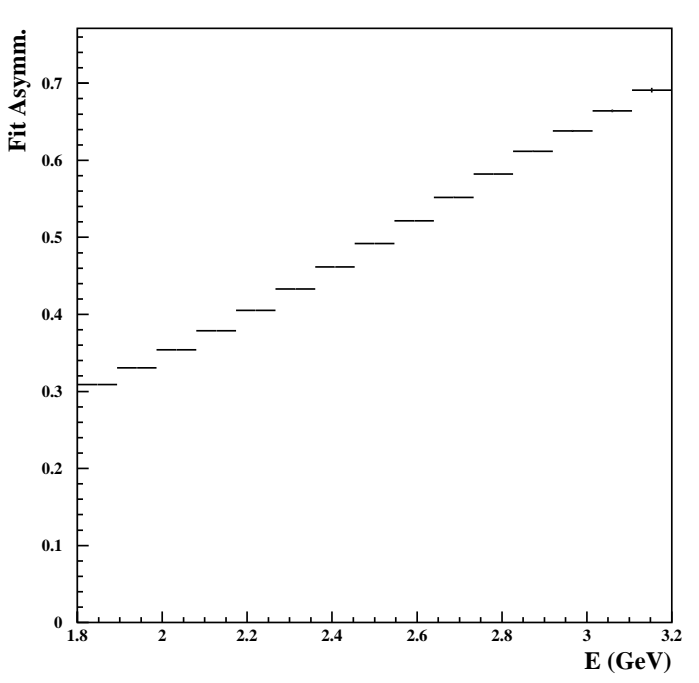

(a) Fit asymmetry vs. low energy threshold.

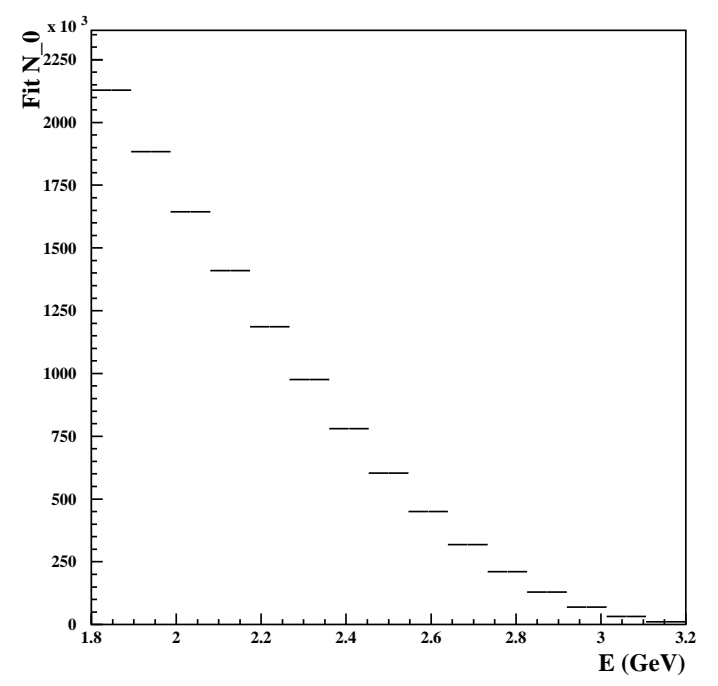

(b) Fit $N$ vs. low energy threshold.

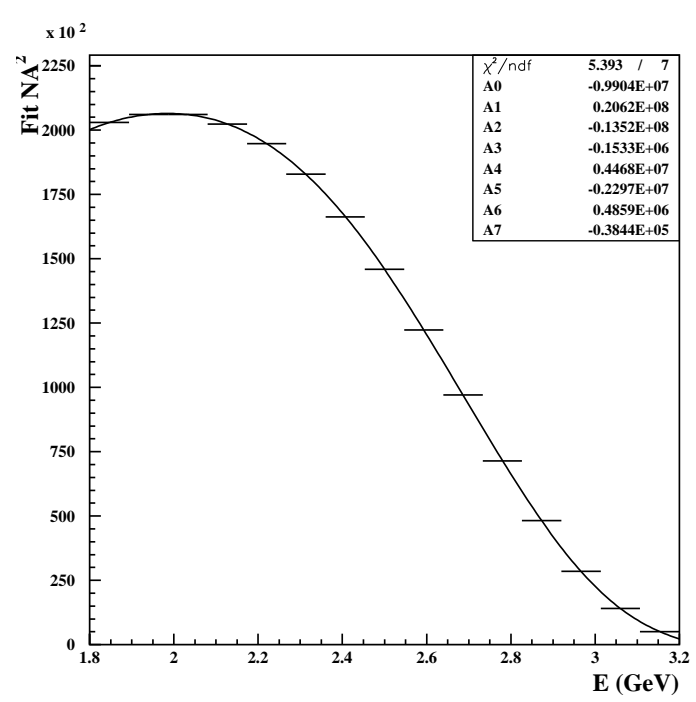

(c) Fit $N A^{2}$ vs. low energy threshold.

Figure 4.3: All plots are for detector 1. The histogram $N A^{2}$ histogram is fit to a $7^{\text {th }}$ order polynomial in order to determine the energy at which the statistical error on the fitted value of $\omega_{a}$ will be minimized. 


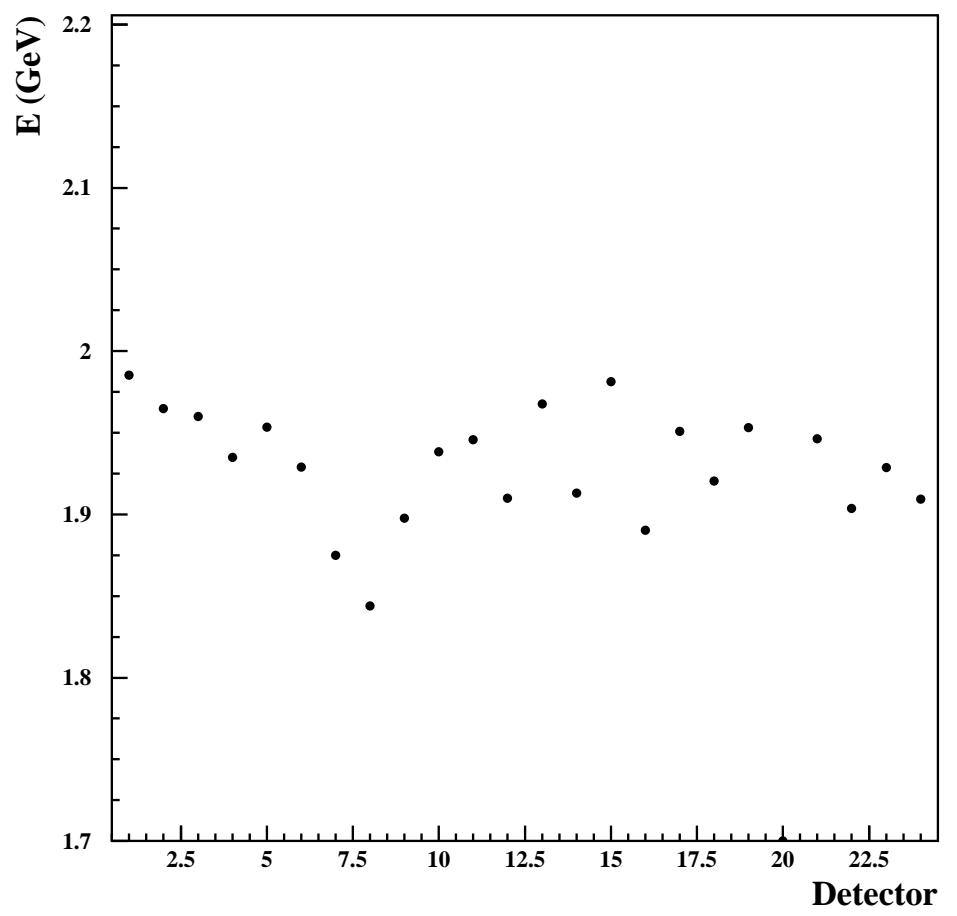

Figure 4.4: Energy threshold values at the peak of $N A^{2}$ vs. $E_{t}$.

\subsubsection{Histogram Filling}

The G2OFF ntuple only stores real number in single precision. Since we are trying to extract a value from the data with better than than $1 \mathrm{ppm}$ precision, the time of the decay electron must be stored in double precision format. The double precision time obtained from the pulse fitting algorithm is stored as two single precision times, one a multiple of $5 \mathrm{~ns}$, the other with a smaller value offset with respect to the first, larger time.

Furthermore, the detectors have slightly different energy responses and therefore have different $g-2$ phases. If we were to add the data from the individual detectors are combined into one time spectrum, the amplitude $(A)$ of the $g-2$ oscillations would be slightly diminished. Therefore, we first fit the non-phase-aligned time spectra at $\sim 100 \mu$ s to extract the $g$-2 phase for each detector. As expected for $\mu^{-}$, all phases are quite close to $\pi$. We then build new time spectra with aligned $g$-2 phases, shifting the times by $\Delta t=\left(\pi-\phi_{0}\right) / \omega_{a}$, where $\omega_{a}=229.067 \mathrm{kHz}$.

The final time spectra are built using 150 ns-wide time bins. Using data in the range 0-675 $\mu \mathrm{s}$, there are a total of 4500 bins per time spectra. The width of the time bin was chosen primarily for its proximity to the cyclotron period of the $g$ - 2 storage ring.

\subsubsection{Removing Pileup From the Data}

When two (or more) decay electrons hit a detector within the pulse reconstruction algorithm's time resolution, the pulses cannot be separated and are treated as one pulse of 


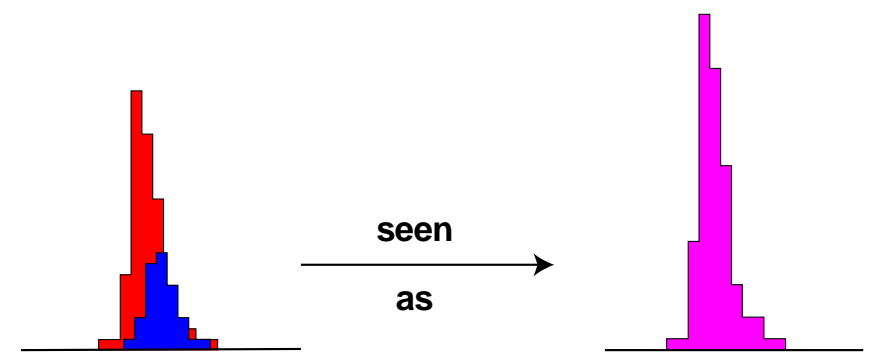

Pileup: Overlapping pulses cannot be resolved

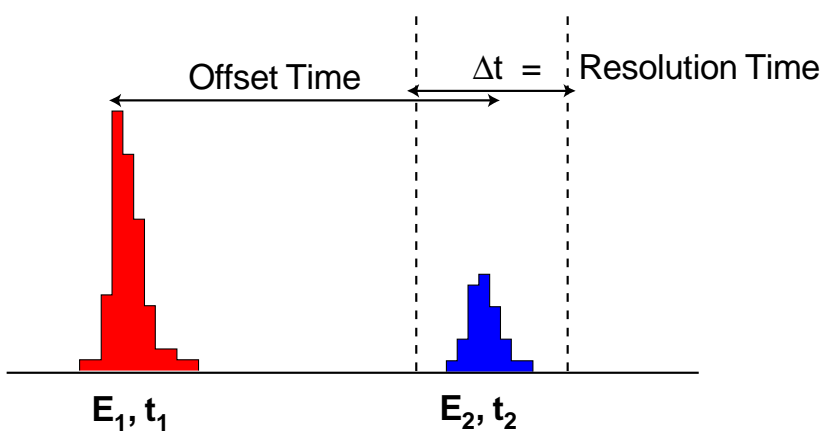

Probrability of having overlapping pulses is the same as having pulses separated by a small offset time ( 10 ns)

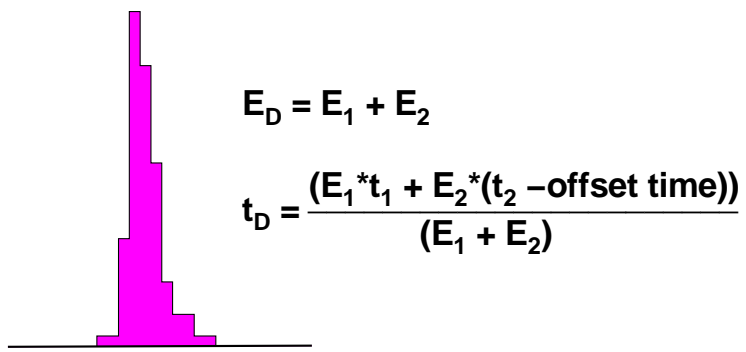

Reconstructed pileup pulse with energy $E_{D}$ and time $t_{D}$

Figure 4.5: Method of pileup construction. 
higher energy. Therefore pileup events either add events to the time and energy spectra (eg: two pulses below the lower energy threshold may add up to a pulse above the lower energy threshold) or subtract events from the time spectrum (eg: two pulses above the lower energy threshold add up to a pulse above the upper energy threshold). Since pileup is also rate-dependent, and since more decay electrons are observed at the peak of the $g$ - 2 cycle than at the bottom, the number of pileup events oscillates at the $g$ - 2 frequency. Furthermore, since pileup events include pulses with a different $g$-2 phase (eg, low-energy pulses), the phase of the pileup $g-2$ oscillations is slightly different from that of the main signal of single events. Therefore pileup events affect the decay electron time and energy spectra as a background term with its own asymmetry and $g$-2 phase. In other words, pileup results in a time-dependent asymmetry and $g$-2 phase, which causes a time-dependent, oscillating fit value of $\omega_{a}$ (often referred to as "phase-pulling"), unless dealt with appropriately.

A method of removing pileup from the time and energy spectra of single decay electron events, developed by Y. Semertzidis, C. Özben [89] and others, involves constructing the pileup time spectrum from the data itself. The approach is based on the principle that the probability that two pulses may overlap is the same as the probability that two pulses will be separated by a small time offset, such as $10 \mathrm{~ns}$. The pileup time and energy spectra may be statistically constructed by looking at "shadow" pulses, or pulses that follow an initial trigger pulse, as described in Fig. 4.5. If a shadow pulse falls within a window (the size of which is determined by the dead-time) some time-distance from the trigger pulse, we use the energies and times of the two pulses to construct a pileup pulse.

In this analysis, we use the pileup construction algorithm derived in [89]. An energydependent dead-time is used to select shadow pulses in the construction of pileup. The energy and time of each constructed pileup pulse, $E_{D}$ and $t_{D}$, are given as

$$
E_{D}=c_{L}\left(E_{1}, E_{2}\right) \times\left(E_{1}+E_{2}\right)
$$

and

$$
t_{D}+\frac{E_{1} t_{1}+E_{2}\left(t_{2}-t_{\mathrm{off}}\right)}{E_{1} E_{2}}
$$

$c_{L}$ is an energy-dependent scale-factor for combining the energies of two single pulses into one double pileup pulse. The energy-dependent dead-time and scale-factor $c_{L}$ (often referred to as the "Logashenko coefficient") were calculated via simulation and provided in a look-up table by I. Logashenko. Typical values for the dead-time and scale factor are $2.9 \mathrm{~ns}$ and 0.96, respectively. The time offset of the shadow pulse search window is set to $12 \mathrm{~ns}$ for all detectors.

The constructed pileup energy and time spectra are then subtracted from the original spectra. Fig. 4.6 shows the energy spectra of a few of the individual detectors before pileup subtraction (ALL) and the energy spectra of the constructed pileup events (PU). Note that the number of pileup events goes negative around $2.6 \mathrm{GeV}$, which means that below this energy, pileup events have a negative contribution to the number of observed decay electrons, whereas above $2.6 \mathrm{GeV}$ pileup events have a positive contribution. The actual energy at which the pileup spectrum crosses zero as a function of detector is shown in Fig. 4.7.

Time spectra of the constructed pileup events using different energy cuts for detector 1 are shown in Fig. 4.8. Note that for the nominal cuts $(1.9<E<3.2 \mathrm{GeV})$, the number of 

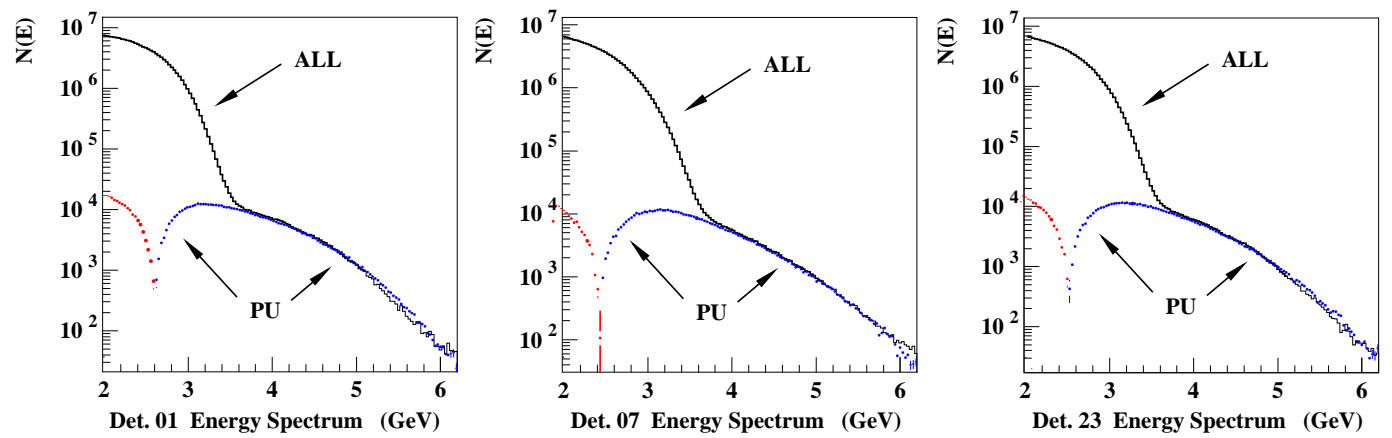

Figure 4.6: Energy spectra for a few of the individual detectors. Since the pileup energy spectrum goes negative near $2.6 \mathrm{GeV}$, we plot the absolute number of pileup events for energies $<2.6 \mathrm{GeV}$.

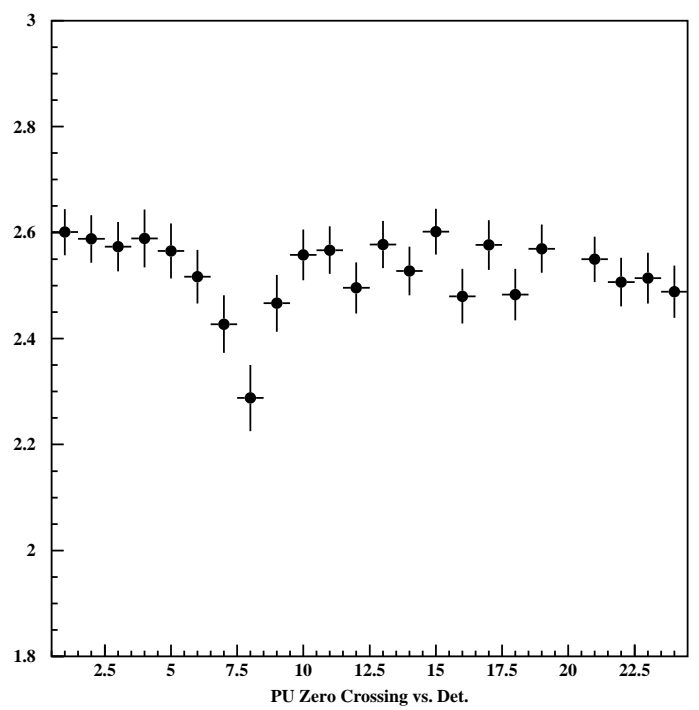

Figure 4.7: Energy at which the number of pileup events crosses zero as a function of detector. 


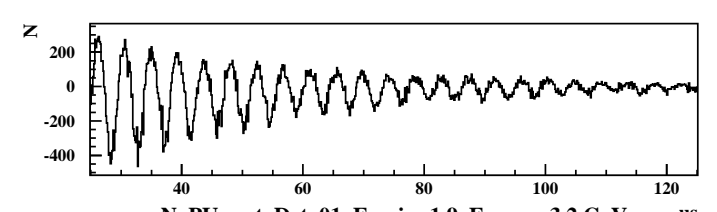

N_PU vs. t, Det. 01, E_min=1.9, E_max $=3.2 \mathrm{GeV}$ us
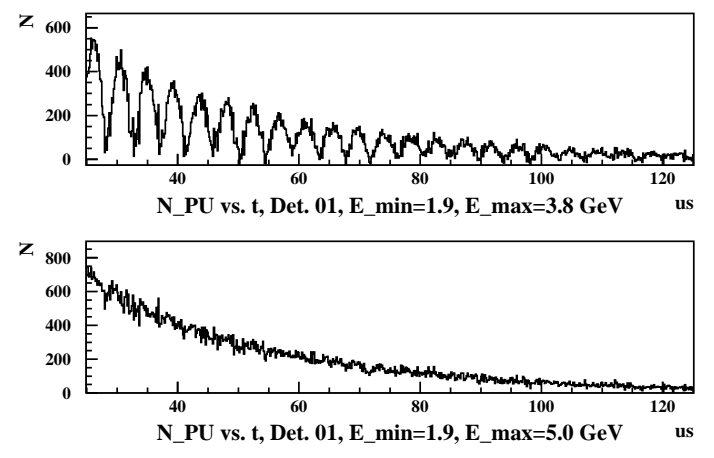

(a) Lower energy threshold is fixed, upper energy threshold is varied.
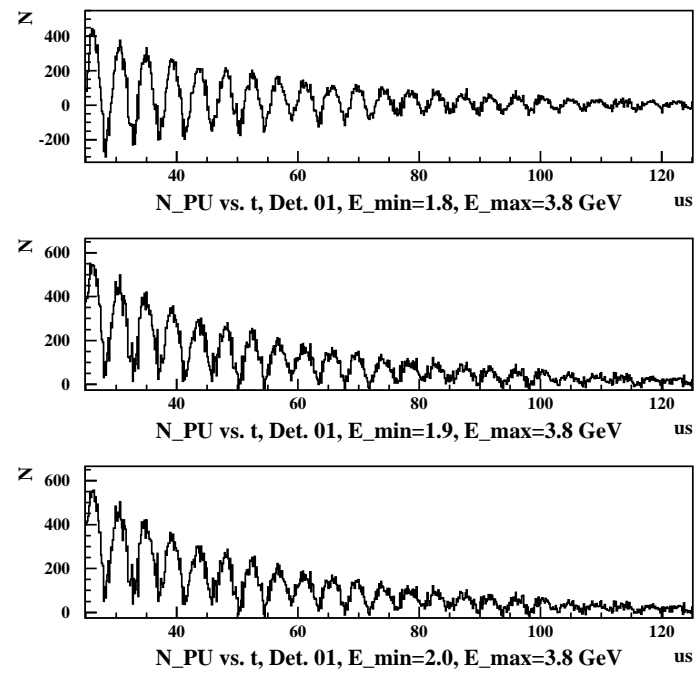

(b) Upper energy threshold is fixed, lower energy threshold is varied.

Figure 4.8: Constructed pileup time spectra for detector 1 using different energy cuts.

pileup events as a function of time oscillates near zero and therefore the integrated number of events is very close to zero. This is not surprising, since if one looks at Fig. 4.6 it is clear that from 1.9-3.2 GeV, nearly half the constructed PU events contribute negatively (the lower energy events) and the other half contribute positively (the higher energy events).

\subsubsection{Energy Scale Changes}

Since $N, A$ and $\phi$ are energy-threshold dependent, a changing detector energy response during the fill results in $\omega_{a}$ phase-pulling. We refer to changes in the observed energy spectrum as "energy scale changes". Since the PMT tubes of the electron detectors are turned on 6-20 $\mu$ s after every injection, the gain on the tubes is not stable until $\sim 10 \mu \mathrm{s}$ later. However, gain may not be the only cause of energy scale changes. The high level of neutron background produced at injection causes small change in the fitted energies of the decay electron pulses. Also, the pulse reconstruction algorithm may be rate-dependent. As a rule of thumb, in order to claim an energy-scale change systematic uncertainty less than $0.1 \mathrm{ppm}$, changes in energy-scale should be less than $0.2 \%$ over $200 \mu \mathrm{s}$.

One way to get a handle on energy scale changes is to look at the average energy of pulses as a function of time in the fill. Because the average energy oscillates at the $g-2$ frequency, the data must be averaged over a $g$-2 cycle. Fig. 4.9 show the normalized average energy vs. time, $\xi(t)$, in the fill for a few detectors. The normalization is performed with respect to the average energy of decay electrons detected after $250 \mu \mathrm{s}$, so that

$$
\xi(t)=\frac{\langle E(t)\rangle}{\left\langle E_{\text {late }}\right\rangle}
$$



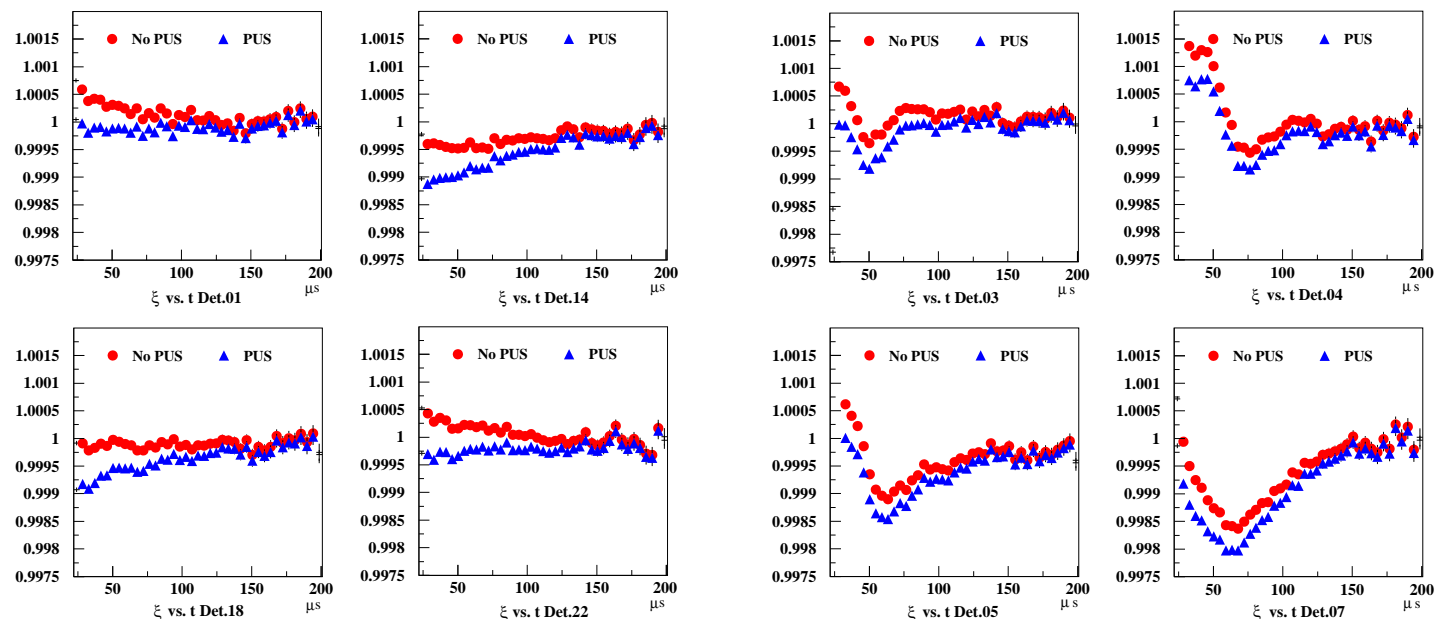

(a) "Well-behaved" detectors.

(b) "Noisy" detectors, near the point of injection.

Figure 4.9: Average energy vs. time after injection. The red circles are $\xi=\langle E\rangle /\left\langle E_{\text {late }}\right\rangle$ for non-pileup-subtracted data, the blue triangles are $\xi$ for pileup-subtracted data.

where

$$
\langle E\rangle=\frac{\sum_{E_{\min }}^{E_{\max }} N\left(E^{\prime}\right) E^{\prime}}{\sum_{E_{\min }}^{E_{\max }} N\left(E^{\prime}\right)}
$$

The circles show the average energy vs. time before pileup subtraction and the triangles show the average energy vs. time after pileup subtraction. We see that at early times pileup raises the average energy by about $0.07-0.08 \%$.

Most changes in average energy after pileup subtraction are less than $0.2 \%$ from $25-200$ $\mu$ s. However of particular concern are detectors 3-7 which show very large changes in $\xi(t)$. Moreover, the shapes of gain vs. time for these detectors not "behave" as those of the other detectors. The origin of these large, odd-shaped changes in average energy during the first $100 \mu \mathrm{s}$ for these detectors was an error in the treatment of island length by the G2OFF production. Because of their close proximity to the injection point, detectors 3-7 see the largest amount of flash, and at early times the calorimeter WFDs digitize continuously. The very long islands of data seen during and just after continuous digitization of the WFDs are broken up into smaller islands in order to fit the individual pulses. However, the pulses have very long tails, and the average pulse shape to which the pulses are fit has a fixed island length. Therefore fits to pulses on islands longer than that of the average pulse shape do not properly account for the pedestal, resulting in a systematic shift in the average energy[90]. Logashenko has shown that one may treat this island-length effect on the extracted energies of pulses as a linear energy-scale change. However, a related systematic error arises from having multiple pulses on a single island, an issue which will be discussed and evaluated in Section 4.4.2. 


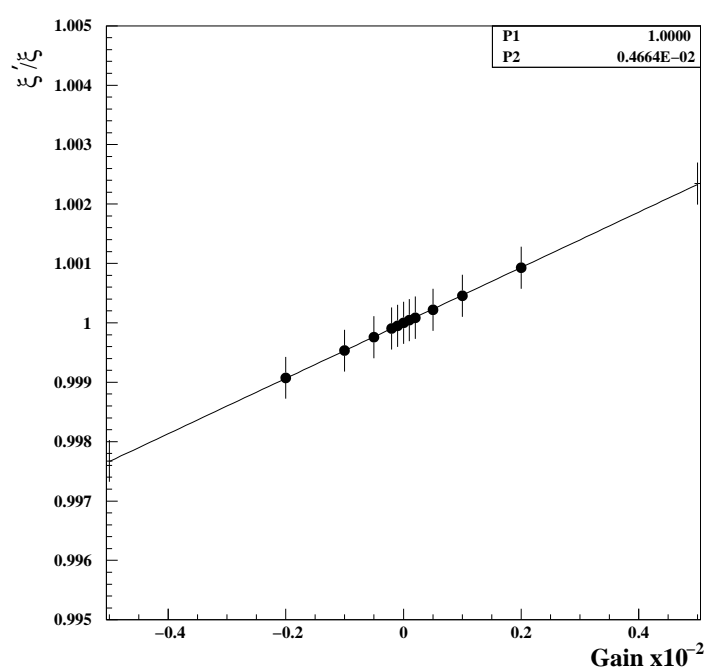

(a) The effect of gain on the average energy for detector 10 .

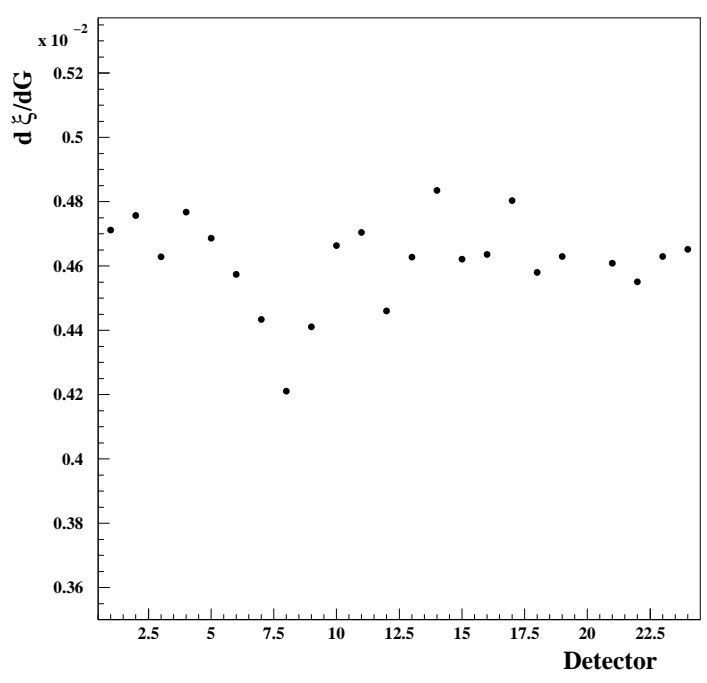

(b) Slope of average energy vs. gain for each detector.

Figure 4.10: To determine the sensitivity of average energy to gain, artificial gains were applied to the data.

We therefore assume that the time dependence of the average energy is caused by some linear effect on the recorded energies of the decay electrons. To correct for energy scale changes, we scale the energy of each pulse according to

$$
E(t)=\frac{E(t)^{\prime}}{1+(\xi(t)-1) / m}
$$

where $E^{\prime}$ is the observed energy of the decay electron at time $t, E$ is the "true" energy of the electron, and $m$ relates a change in average energy to a change in gain. The shape of the gain is parameterized by fitting a polynomial to $\xi(t)$. In practice, the order of the polynomial is chosen 'by eye' such that the function passes through all the data points.

The relation between gain and average energy is determined by applying various software gains to late-time data where energy-scale changes are assumed negligible. The slope $m$ is therefore

$$
m=\frac{\Delta \frac{\left\langle E^{\prime}\right\rangle}{\langle E\rangle}}{\Delta g}=\frac{\Delta \frac{\xi^{\prime}}{\xi}}{\Delta g}
$$

where $\left\langle E^{\prime}\right\rangle\left(\xi^{\prime}\right)$ is the observed (normalized) average energy of the gain-enhanced pulses, $\left\langle E^{\prime}\right\rangle(\xi)$ is the "true" (normalized) average energy of the pulses, and $g$ is the applied gain. The plot on the left of Fig. 4.10 shows the dependency of $\xi^{\prime} / \xi$ on gain for detector 10, and the plot on the right shows the extracted slope, or $m$, for each detector.

Applying the scale factor $(1+(\xi(t)-1) / m)^{-1}$ to each pulse used in the data, we see in Fig. 4.11 a great improvement in the stability of the average energy vs. time. This implies 

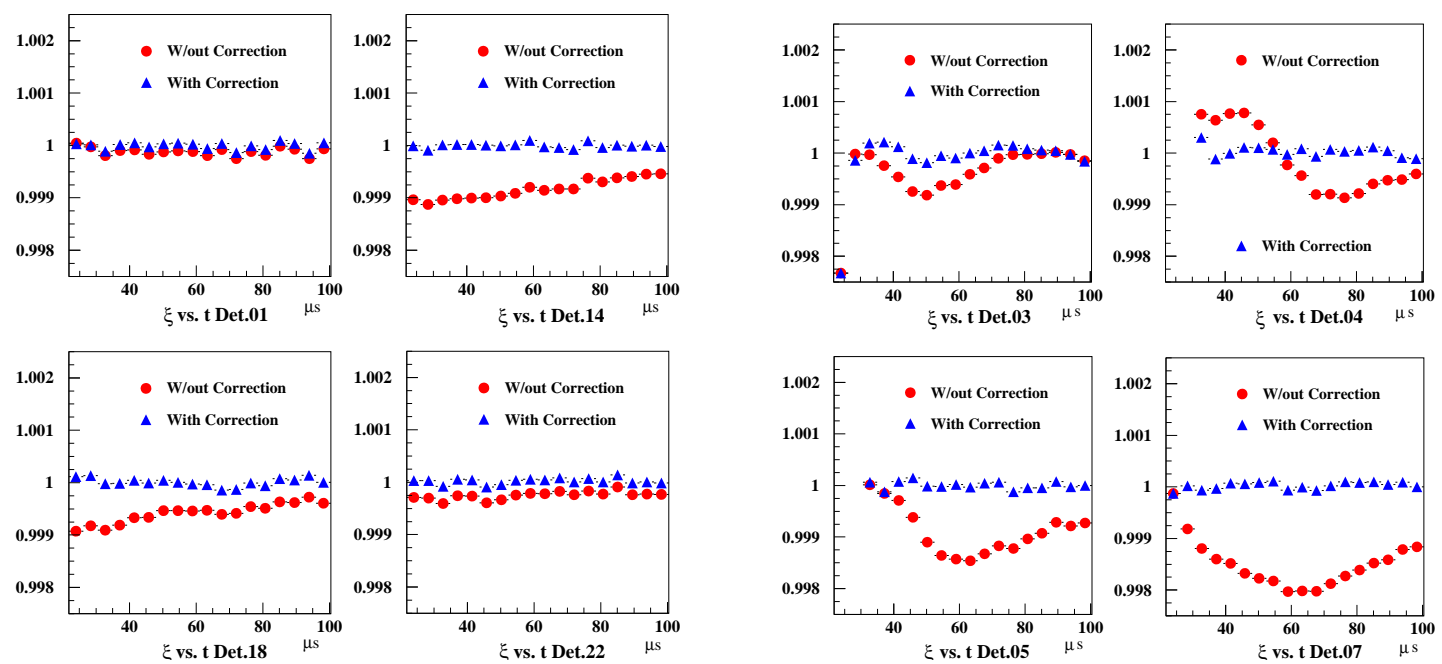

(a) "Well-behaved" detectors.

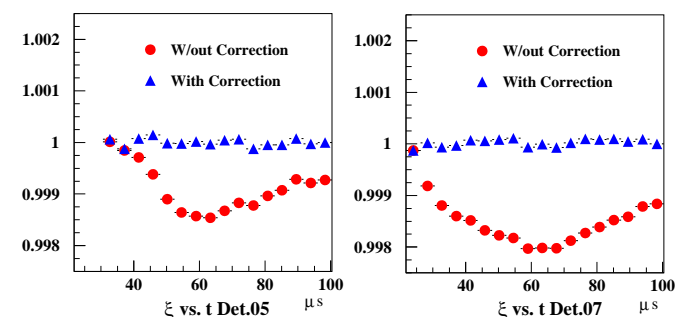

(b) "Noisy" detectors, near the point of injection.

Figure 4.11: Pileup-subtracted average energy vs. time before (circles) and after (triangles) applying an energy-scale change correction.

that whatever is causing the changes in average energy vs. time has a dominantly linear effect on the energies of the pulses. If this were not the case, if the effect were non-linear, then applying a gain correction would not flatten out the average energy vs. time.

However, although the gain correction manages to flatten out the average energy vs. time of the decay electrons, this does not necessarily mean that applying the gain correction is the proper thing to do. A better judge of whether or not one should make the correction is the time spectrum itself, or rather, the fit to the time spectrum. Although the fit to the data has not yet been described, here we show results of fits to justify the application of the gain correction.

Fig. 4.12 shows the fit $\chi^{2} /$ d.o.f. for detector 25 when applying a $-1 \times$ correction (open circles), no correction (triangles) and a $1 \times$ correction (closed circles). We see that for the low-n data set that there is a significant improvement in the fit $\chi^{2} /$ d.o.f. when we apply the gain correction. The improvement is considerably smaller for the high-n data set, the reason for which has not been determined. As expected, the fits get significantly worse when we magnify the gain in the data by applying a $-1 \times$ correction.

Another parameter to investigate is the asymmetry. Since asymmetry is energy dependent, the fit asymmetry will not be constant versus fit time if the average energy changes with time. We see in Fig. 4.13 that when we do apply the correction (middle plots) the fit asymmetry is more or less stable vs. time. However it does trend downward over time. When the correction is applied (bottom plots), the asymmetry is still more or less stable vs. time, but now it trends upward over time.

Based on the facts that the average energy vs. time is constant, the fit $\chi^{2} /$ d.o.f. improves 


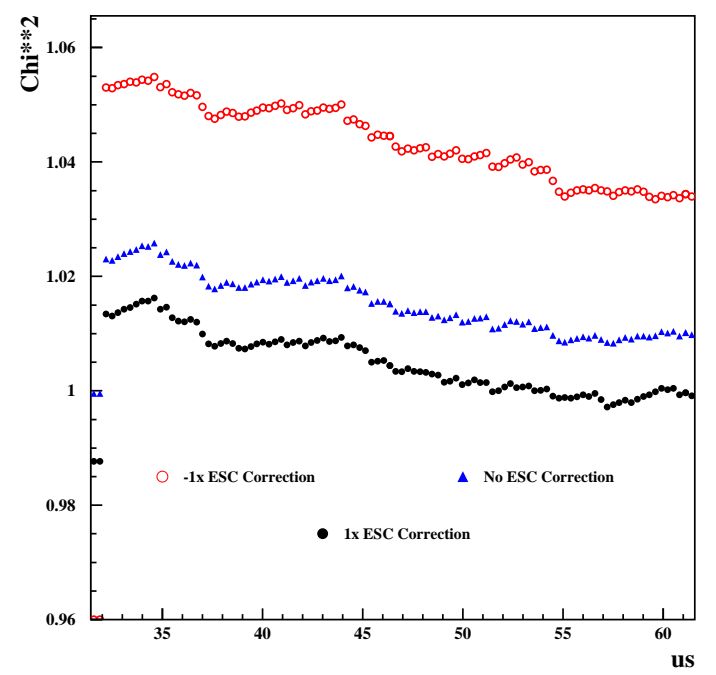

(a) Low-n data set.

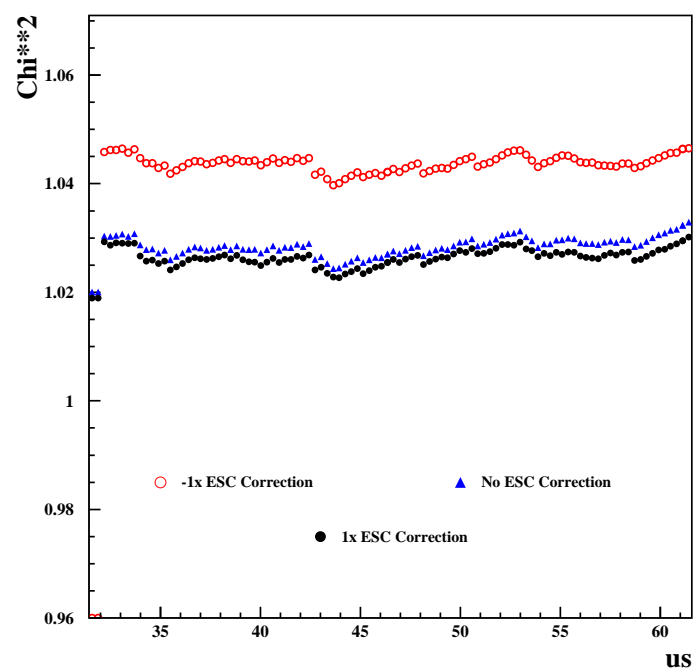

(b) High-n data set.

Figure 4.12: Comparison of fit $\chi^{2} /$ d.o.f. vs. fit time between between un-corrected and gain-corrected data.
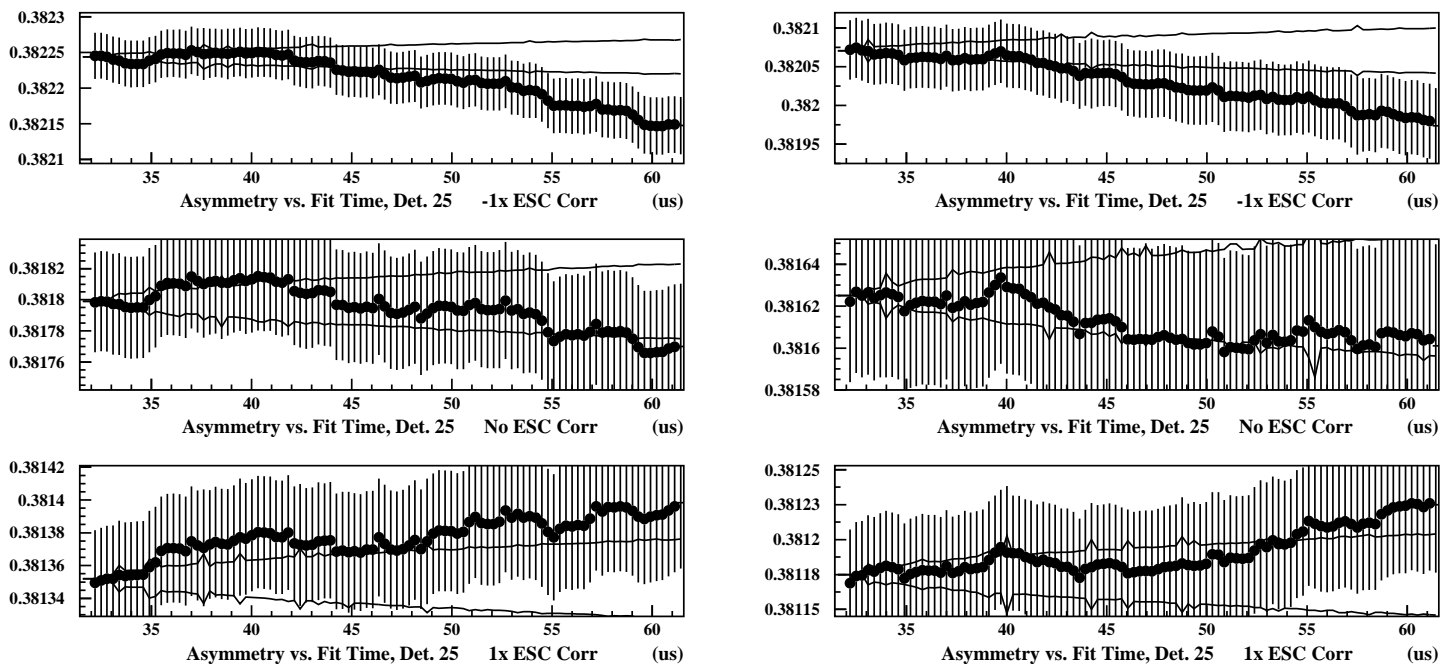

(a) Low n-value data.

(b) High n-value data.

Figure 4.13: Comparison of fit asymmetry vs. fit time between uncorrected data sets (top) and gain-corrected data sets (bottom). 

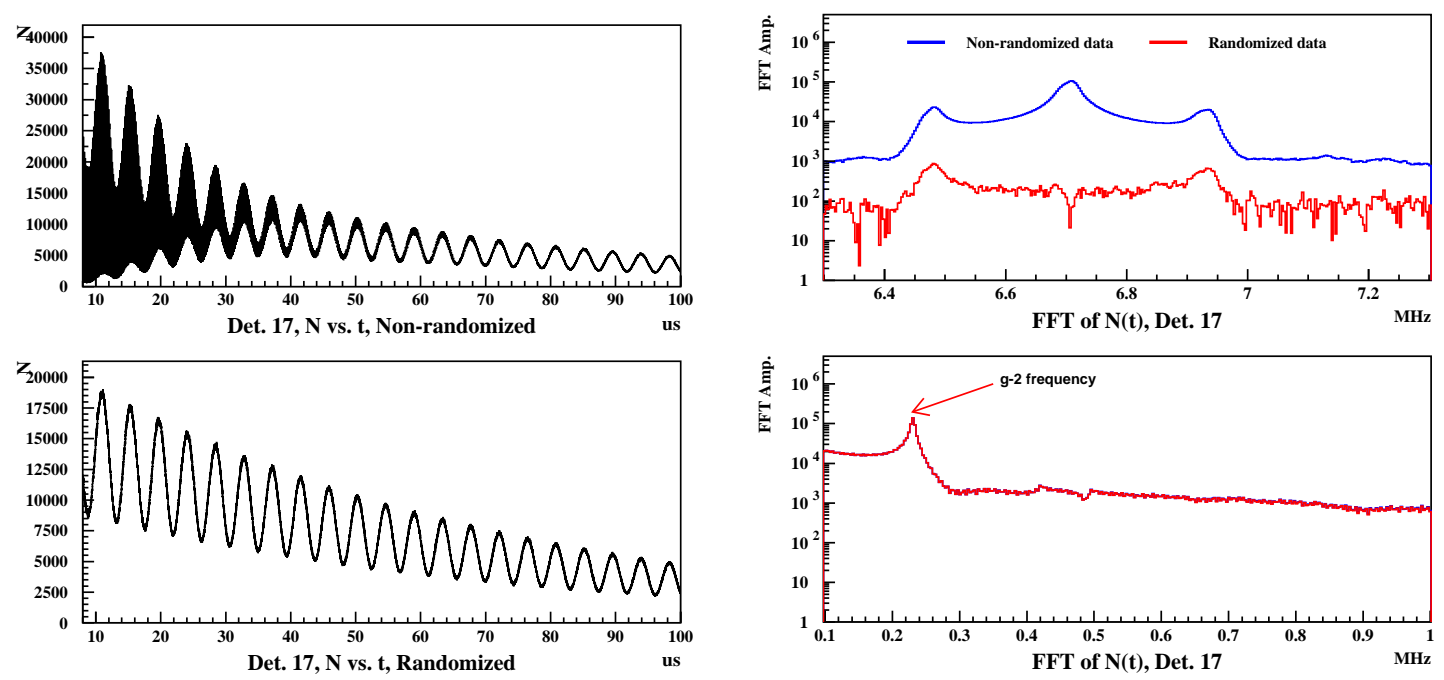

(a) Time spectra of non-randomized data (top) and randomized data (bottom) for detector 17 .

(b) FFT spectra of non-randomized data (blue) and randomized data (red) for detector 17 .

Figure 4.14: Comparison of time and frequency spectra of fill-randomized data and nonrandomized data.

and the fit asymmetry is flat vs. time after we apply a gain correction to the data, we use gain-corrected data to obtain our final fit values for $\omega_{a}$. The systematic error from uncertainties in the corrections are discussed in Section 4.4.2.

\subsubsection{Beam Debunching and Fill Randomization}

Because the muon beam is injected in bunches of width $\sim 20 \mathrm{~ns}$, immediately after injection the stored muons are not distributed uniformly around the storage ring. However, within $50 \mu \mathrm{s}$ the bunch-structure of the injected muon beam is greatly diminished. Debunching occurs because, although all muons travel at 0.9994c, the less energetic muons at smaller radii have a shorter cyclotron period than the more energetic muons. Therefore the less energetic muons actually catch up to and pass the more energetic muons.

The bunching of the beam results in a modulation of the counting rate at early times:

$$
N(t)=N_{5}(t) * f_{\mathrm{bb}}(t)
$$

where

$$
f_{\mathrm{bb}}(t)=A_{\mathrm{bb}}(t) * \cos \left(\omega_{\mathrm{cyc}} t+\phi_{\mathrm{cyc}}\right)
$$

where bb stands for "bunched beam", $A_{\mathrm{bb}}(t)$ describes the decaying envelope of the bunched structure of the muons in the storage ring, and $\omega_{\text {cyc }}$ is the cyclotron frequency. However, because the cyclotron frequency is so much higher than that of the $g$-2 frequency, instead of modifying our fit function we can effectively filter out the cyclotron frequency from the 


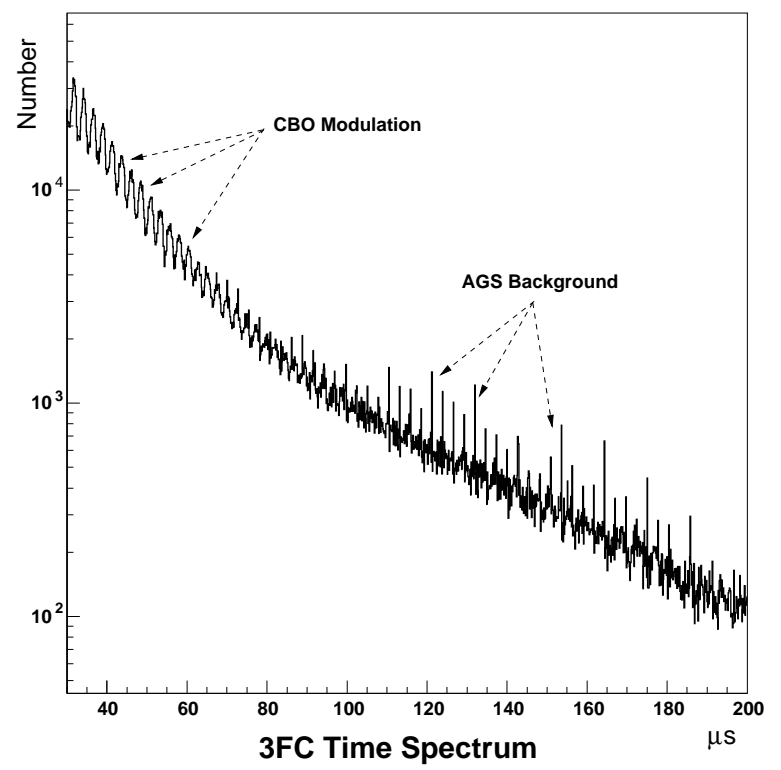

Figure 4.15: Number of three-fold coincidences (3FCs) as a function of time in the low-n 2001 data. The oscillations at early times after injection are at the CBO frequency, and the sharp spikes in the observed number of 3FCs are an artifact from the AGS background.

data by randomizing the time for T0 in each fill. Since the cyclotron period is very close to $149.185 \mathrm{~ns}$, adding random times generated from a uniform distribution between -74.5925 to $+74.5925 \mathrm{~ns}$ for each fill washes out the effect of the bunched beam. Fig. 4.14 shows the FFT of the time spectra of the data from detector 17 with and without randomization. The data are from between 0 and $200 \mu \mathrm{s}$, and is stored in five ns-wide bins. We see that while the amplitude of the cyclotron frequency peak is reduced by 3 orders of magnitude, the amplitude of the $g-2$ frequency peak is unaltered by the fill randomization procedure. In the end, five histograms are built with different random numbers in each fill, and the results of the final value for $\omega_{a}$ is taken from the average of the five fits.

\subsubsection{Muon Losses}

As discussed in Section 2.4.2, beam dynamics resonances can cause muons to be lost before they decay. Losses distort the assumed exponential shape of the decay electron time spectrum, resulting in unstable fit parameters, and a systematic error on the fit value of $\omega_{a}$. In principle, if the functional form of the loss rate were known, this effect could be eliminated by modifying the fit function appropriately.

To this end, a method of detecting lost muons was developed using coincidences in three sequential FSD detectors. The details of this three-fold coincidence (3FC) approach are found in [91]. Simulations have shown that nearly a third of the muons exiting the storage ring pass through three detectors. Although a much larger fraction (60-70\%) of lost muons pass through two detectors (a two-fold coincidence, or 2FC), the dominant decay electron background is eliminated more efficiently using 3FCs. 
The rate of 3FCs in the low-n data set is shown in Fig. 4.15. The time dependence appears to have two exponential components, one with a $\sim 20 \mu$ s lifetime that dominates at early times after injection, the other with a $\sim 50 \mu$ s lifetime that dominates from $\sim 100-200$ $\mu$ s after injection. The large spikes seen in the number of 3FCs after $80 \mu \mathrm{s}$ are caused by AGS background, or "flashlets". Like true lost muons, the particles in the AGS background deposit too little energy in the calorimeters to be observed in the WFDs, but are observed by the FSDs (that is, they are minimum ionizing).

The CBO oscillations in the rate of 3FCs at early times after injection implies that there is a preferred location in the storage ring for the muons to be "lost". The relative number of 3FCs depends strongly on detector location, and most 3FCs are observed approximately $90^{\circ}$ downstream of the inflector. Since the vacuum wall in the inflector region is closer to the storage aperture than anywhere else, it is likely that muons strike the wall, scatter, lose energy and are observed by the detectors downstream.

Having determined the time dependence of the lost muons, the five-parameter fit function is extended to a six-parameter fit function:

$$
N_{6}(t)=N_{0} e^{-t / \tau}\left(1+A \cos \left(\omega_{a} t+\phi\right)\right) e^{-A_{L} F_{L}(t)}
$$

where $A_{L}$ is the fraction of total losses,

$$
F_{L}(t)=\int_{t_{0}}^{t} N_{3 F C}\left(t^{\prime}\right) d t^{\prime}
$$

$t_{0}$ is the fit start time, and $N_{3 F C}(t)$ is the number of $3 \mathrm{FCs}$ as a function of time normalized such that $F_{L}(\infty)=1$. This approach is different from others used in the past, where the functional form of the muon loss term was assumed to be either a Gaussian or exponential, and both the amplitude and the lifetime were determined from fits. Use of the measured $3 \mathrm{FC}$ time spectrum substantially improves the $\chi^{2} /$ d.o.f. of fits to the data, and is employed by all fits to $N(t)$ in the 2001 analysis.

The method has its drawbacks. The 3FC time spectrum is limited to only those lost muons that pass through three sequential detectors, and is not measured everywhere around the storage ring. Indeed, the shape of the $3 \mathrm{FC}$ time spectrum varies from detector to

detector. Therefore it is questionable that the $3 \mathrm{FC}$ time spectrum sufficiently represents the true loss spectrum. The insensitivity of the ratio to the shape of the muon loss time spectrum was one of the motivations in choosing the ratio method over conventional direct fits to the decay electron time spectrum.

\subsubsection{The Ratio Method}

The ratio method was originally derived by Y. Orlov and first implemented by J. Kindem [47]. Since then, the ratio method has been used in the analysis of the 1999 data by L. Duong [66] and the 2000 data by B. Bousquet. While this analysis is based on previous works, we have extended the ratio method to include CBO background terms.

Figs. 4.16-4.17 show how the ratio method works. The basic idea is to divide out the exponential decay in the time spectrum. One quarter of the data is shifted forward in time by half a $g-2$ cycle, another quarter of the data is shifted backward in time by half a $g$ - 2 


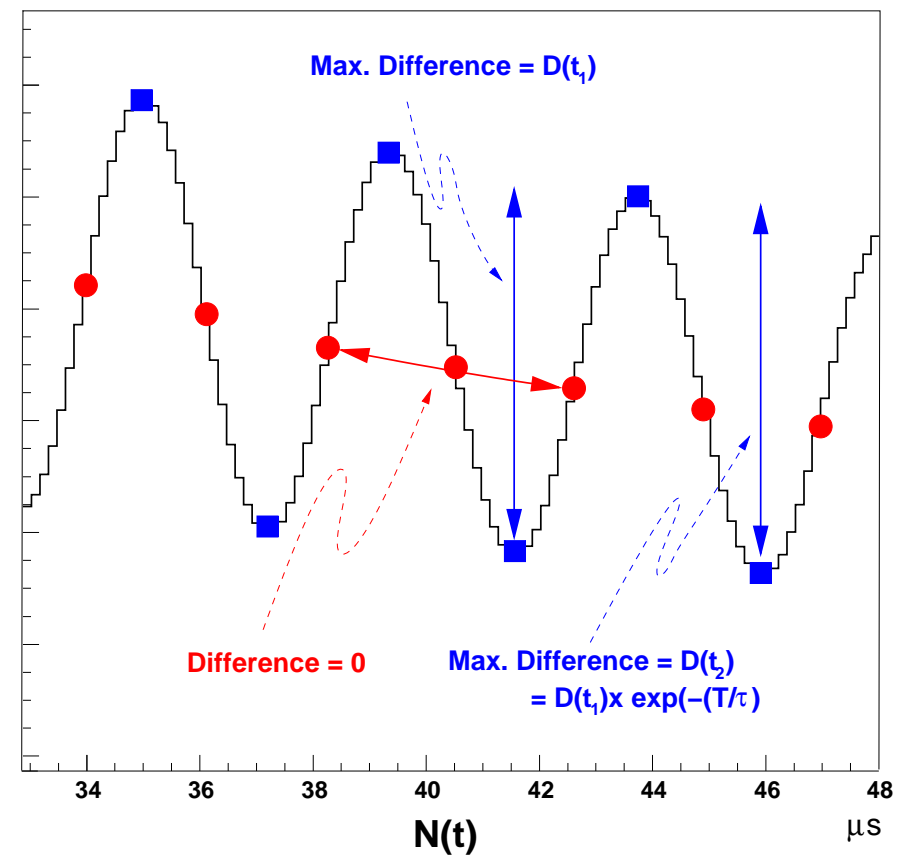

Figure 4.16: In the ratio method, one quarter of the data is shifted back in time by half a $g-2$ period, another quarter of the data is shifted forward in time by half a $g-2$ period, and half of the data is unshifted. At the $g$-2 zero crossings, the difference between the sum of the shifted data and the unshifted data is at a minimum (nearly zero), whereas at the peak (or trough) of the $g-2$ oscillation, the difference is maximal $(\simeq A)$.

cycle, and the remaining one-half of the data remains untouched. We define

$$
U(t)=u_{+}(t)+u_{-}(t), \quad V(t)=v_{1}(t)+v_{2}(t)
$$

where

$$
u_{+}=N\left(t+T_{a} / 2\right), \quad u_{-}=N\left(t-T_{a} / 2\right) \quad \text { and } \quad v_{1,2}=N(t)
$$

and $T_{a}$ is the assumed $g$-2 frequency.

As shown in Fig. 4.16, the difference $U(t)-V(t)$ is nearly zero at a $g$-2 zero crossing, whereas it is maximal at the peak or trough of the $g$-2 cycle. However, although one $g-2$ period later the difference is still zero at the zero crossing, the exponential decay in the number of counts causes the difference to be slightly smaller at the peak or trough than the earlier difference. Therefore the difference is an exponentially decaying cosine, shown in the top left plot of Fig. 4.17. Note that the sum of all data, $U(t)+V(t)$, is simply an exponential decay, shown in the bottom left plot of Fig. 4.17. Taking the ratio of the difference over the sum, the five-parameter function

$$
N_{5}(t)=N_{0} e^{-t / \tau}\left(1+A \cos \left(\omega_{a} t+\phi\right)\right.
$$




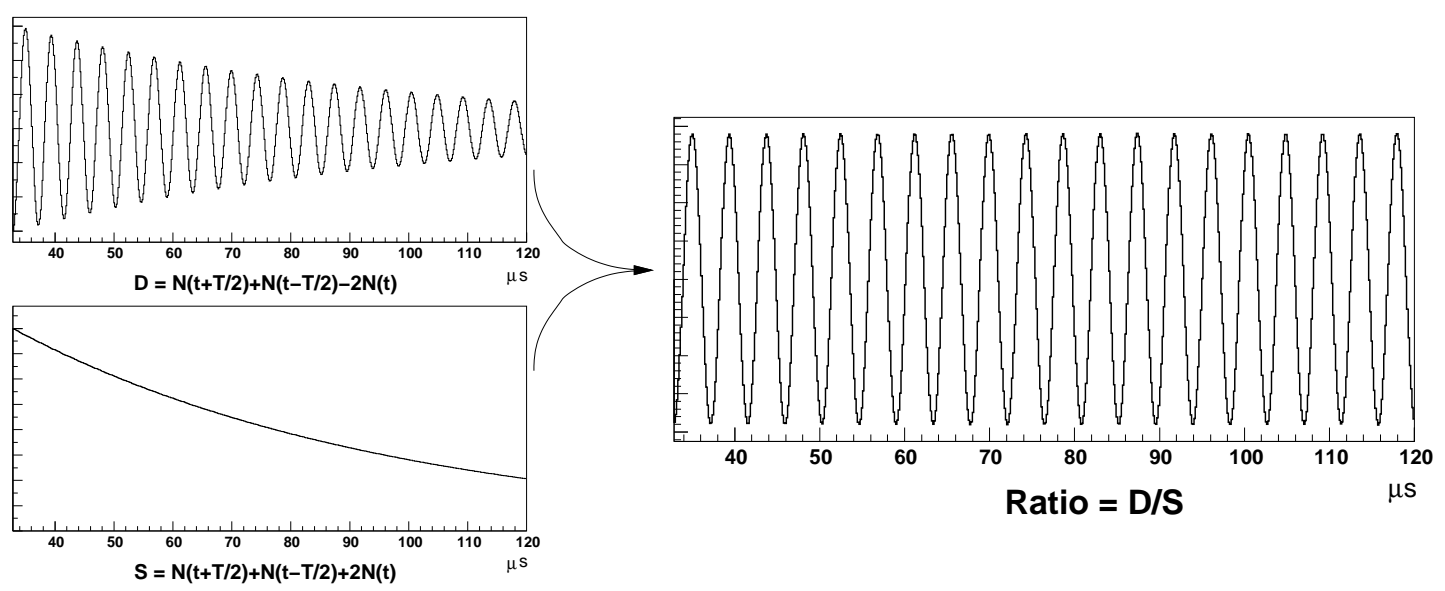

Figure 4.17: The difference of the sum of the time-shifted data and the unshifted data results in an exponentially decaying cosine. The sum of all time-shifted data and unshifted data results in a exponential of the same lifetime. The ratio of the difference $(D)$ over the sum $(S)$ is simply a cosine.

is reduced to the three-parameter function

$$
r(t)=\frac{U(t)-V(t)}{U(t)+U(t)}=A \cos \left(\omega_{a} t+\phi\right)+C_{1}
$$

where

$$
C_{1}=\frac{1}{16}\left(\frac{T_{a}}{\tau}\right)^{2} \simeq 2.87 \times 10^{-4}
$$

A full derivation of the three-parameter ratio function is found in Appendix B.1. Fig. 4.18(a) shows the time spectrum of all decay electrons used in this analysis, in 150 ns-wide bins. The number of counts is plotted on a log scale. In order to make sure the $g$-2 oscillations are visible on this scale, the data are wrapped around the time axis every $90 \mu \mathrm{s}$. The corresponding ratio spectrum of the same data is shown in Fig. 4.18(b).

In practice, one must know the spin precession frequency to within $\sim 10 \mathrm{ppm}[66]$ in order to make a ratio spectrum with sufficiently negligible higher order terms (see Appendix B.1). We have verified that the value of $T_{a}=2 \pi / \omega_{a}$ obtained from late-time fits to the data agree within errors of the value used in this analysis.

The ratio method requires four time spectra to be built. For each pulse, a random integer between 0 and 3 is generated from a flat distribution obtained using the RANLUX routine at luxury level 4 . The four numbers $0,1,2$, and 3 determine into which of the four histograms the pulse time gets inserted: if it is 0 , the pulse time is shifted by $+1 / 2$ the $g$ - 2 period and is inserted into the $u_{+}$histogram, if it is 1 , the pulse time is shifted by $-1 / 2$ the $g-2$ period and is inserted into the $u_{-}$histogram, and if it is either 2 or 3 , the pulse time is not shifted and is inserted to either the $v_{1}$ or the $v_{2}$ histogram respectively. The pileup subtracted $u_{+}, u_{-}, v_{1}$, and $v_{2}$ time spectra are later summed over runs, and the ratio time spectrum is constructed using Eq. 4.16. 


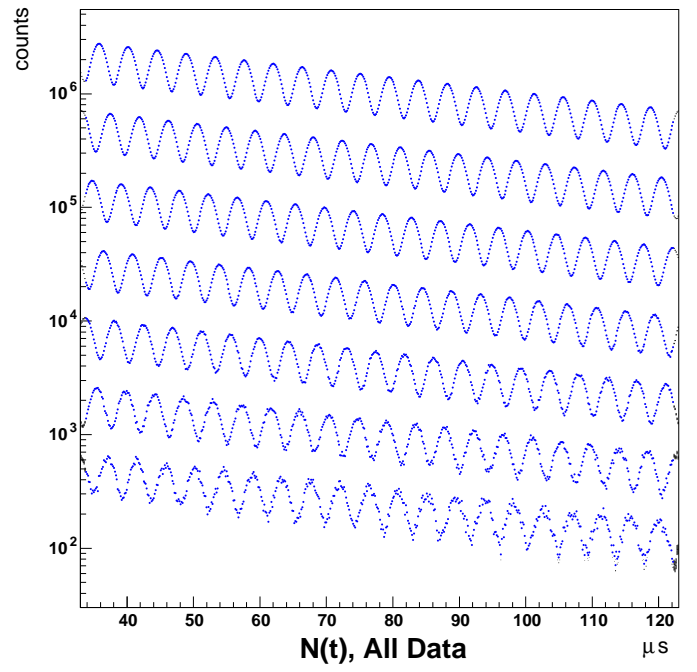

(a) Original spectrum, $N(t)$.

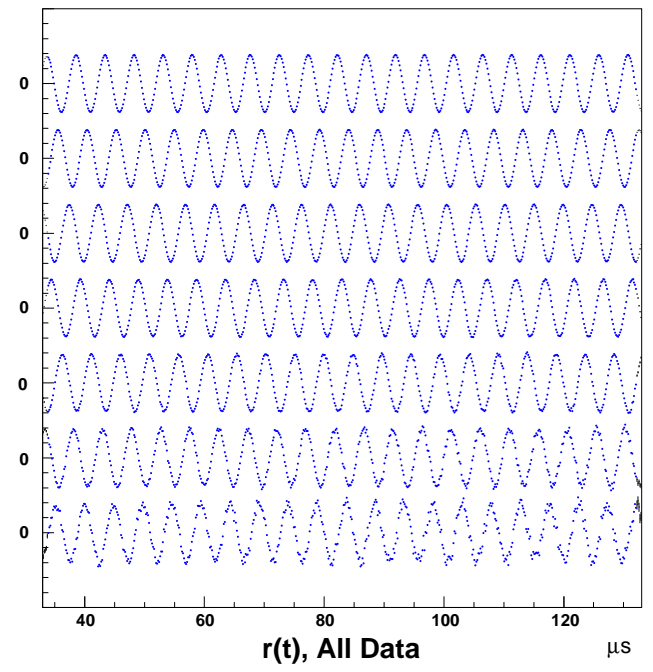

(b) Ratio, $r(t)$.

Figure 4.18: Original $N(t)$ spectrum and ratio function, both produced using all 2001 data. Note that $N(t)$ is plotted on a log vertical scale. The horizontal axis wraps around every $90 \mu \mathrm{s}$, starting from $33 \mu \mathrm{s}$ after injection.

There are several advantages in using the ratio to obtain $\omega_{a}$. The most obvious is the reduction in the number of fit parameters, from five to three. This is related to the most powerful advantage of the ratio method, the decreased sensitivity of the fit $\chi^{2} /$ d.o.f. and $\omega_{a}$ to changes in number of counts over time scales greater than the $g$ - 2 period $(\sim 4.4 \mu \mathrm{s})$. Therefore, some background terms, such as muon losses, may be neglected when fitting the data.

To see this, we have simulated a single time spectrum of $3.6 \times 10^{9}$ decay electrons according to the five-parameter function, but with muon losses included. The variable $R$ is defined as $R=1-\omega_{a} / \omega_{a_{0}}$, where in the simulation $\omega_{a_{0}}=2 \pi \times 0.229067 \mathrm{rad} / \mathrm{s}$ and $R=15$ ppm. The other variables in the simulation were $\tau=64.4 \mu \mathrm{s}, A=0.4136$, and $\phi=1.5$ $\mathrm{rad}$. The muon loss time spectrum used is an exponential with a lifetime of $25 \mu \mathrm{s}$ and an amplitude of $1 \%$ at $\mathrm{t}=0$. The data are binned in $150 \mathrm{~ns}$ steps. The ratio is constructed from the same data. The time spectrum is then fit to the simple five-parameter function, and the ratio is fit to the simple three-parameter function. Fig. 4.19 shows the values of the individual parameters and $\chi^{2} /$ d.o.f. as a function of fit start time. The dashed lines are the correlated error bands with respect to the first fit result; statistically, for an acceptable fit function, one expects $\sim 67 \%$ of the fit results to fall within these lines. From the very poor fit $\chi^{2}$ /d.o.f. at early times and from the time dependence of the fit parameters, it is clear that the five-parameter fit function fails to describe the decay electron time spectrum. On the other hand, the results from the three-parameter fit to the ratio seem unperturbed by muon losses. 

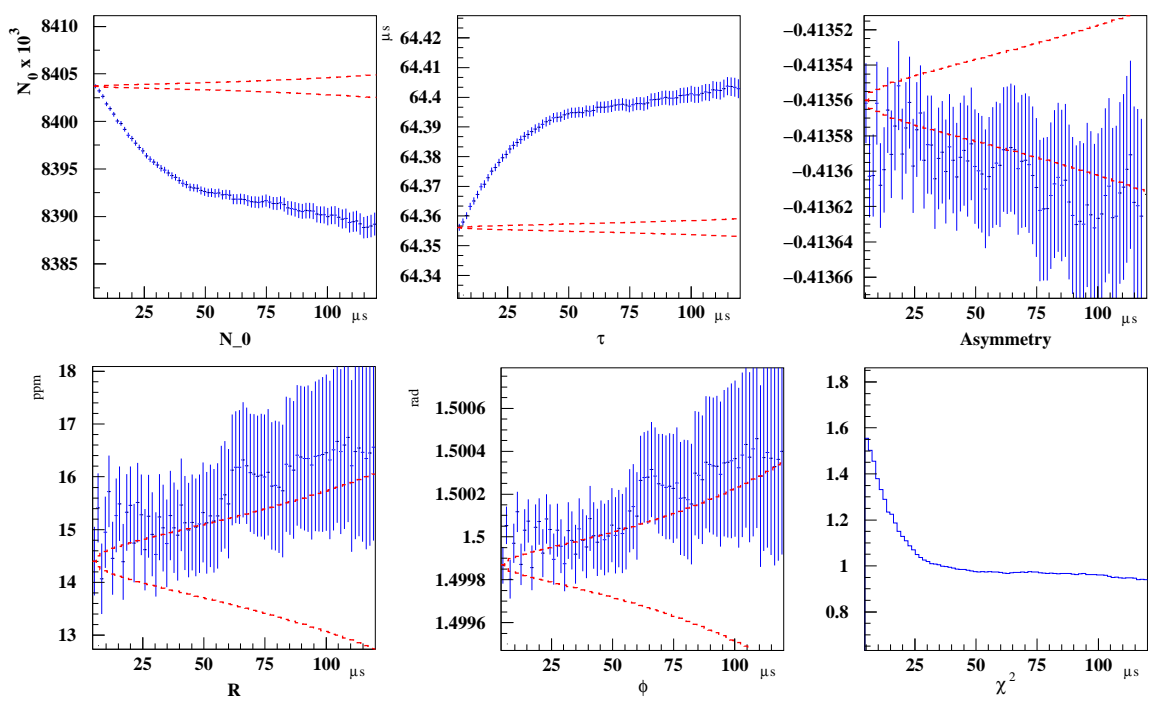

(a) Five-parameter fit results to the decay electron time spectrum.
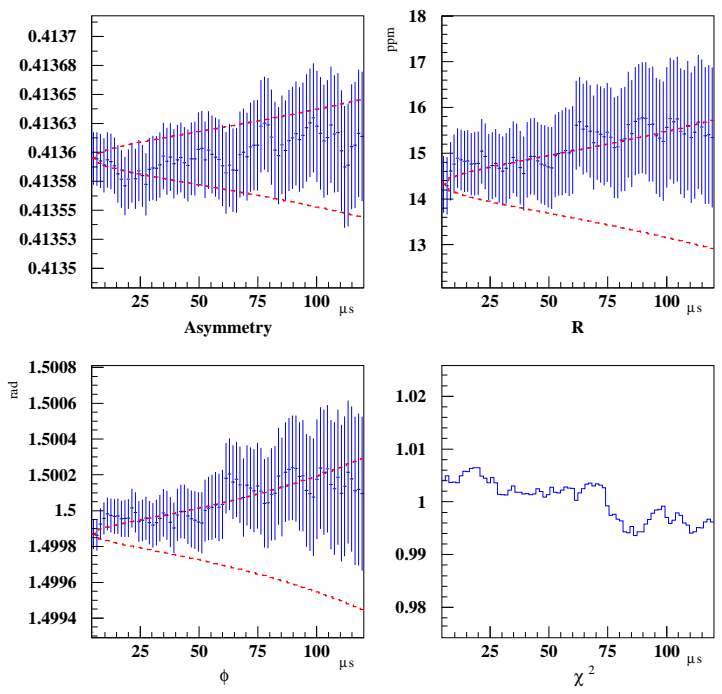

(b) Three-parameter fit results to the ratio.

Figure 4.19: Fit results to simulated data. The simulated spectrum is derived from a fiveparameter function, but with muon losses included. The input values of the simulated time spectrum are $\tau=64.4 \mu \mathrm{s}, R=15 \mathrm{ppm}, A=0.4136$ and $\phi=1.5 \mathrm{rad}$. Both the decay electron time spectrum and the ratio are fit to their corresponding functions, and the fitted values of the parameters are plotted as a function of fit start time. The dashed lines are the correlated error bands. 


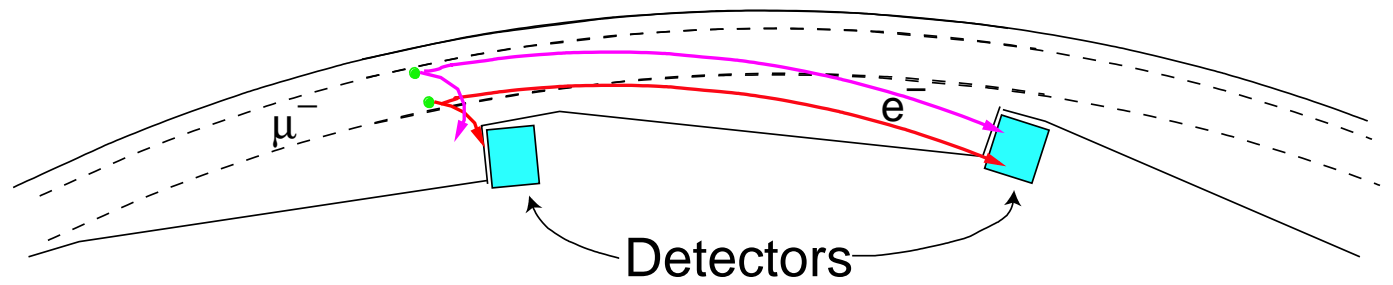

Figure 4.20: Schematic of muon decay inside the storage region.

Although the ratio fit is much less sensitive than a simple fit to slowly varying backgrounds, it is still sensitive to changes in the asymmetry and phase. Therefore, if lost muons have a different average asymmetry or phase than the rest of the stored muon population, a systematic shift in the fitted value of $\omega_{a}$ will be observed. Furthermore, effects such as energy scale changes, pileup, and $\mathrm{CBO}$, which all affect $N_{0}, A$ and $\phi$, must still be taken into account with the ratio method. As previously discussed, energy scale changes and pileup are both corrected for before fitting the data. Therefore this analysis attempts to account properly for all CBO effects by modifying the ratio fit function.

\subsection{Fits to the Data}

\subsubsection{CBO Parameters}

\section{CBO Frequency and Lifetime}

The acceptance of the electron detectors depends on the location of the muon decay vertex in the ring, as well as the energy of the decay electron, as demonstrated in Fig. 4.20. This dependence arises from geometric considerations: for example, low-energy electrons emitted at smaller radii are more likely to hit a detector than low-energy electrons emitted at larger radii. Furthermore, because electrons born at larger radii take longer to reach a detector than electrons born at smaller radii, there is a difference in the observed time of decay, which appears as a difference in phase. From the point of view of a single detector, the muon beam is oscillating in and out radially at the CBO frequency, and the average energy of the decay electrons oscillates at the same frequency. Since $N_{0}, A$ and $\phi$ are all energythreshold dependent, these terms will also oscillate at the CBO frequency. We therefore find that the decay electron counting rate is modified from

$$
N(t)=N_{0} e^{-t / \tau_{\mu}}\left(1+A \cos \left(\omega_{a} t+\phi\right)\right)
$$

to

$$
N(t)=N_{0}\left(1+A_{N_{\text {cbo }}}(t)\right) e^{-t / \tau_{\mu}}\left(1+A\left(1+A_{A_{\text {cbo }}}(t)\right) \cos \left(\omega_{a} t+\phi+A_{\phi_{\text {cbo }}}(t)\right)\right)
$$

where the functions $A_{N_{\mathrm{cbo}}}(t), A_{A_{\mathrm{cbo}}}(t)$ and $A_{\phi_{\mathrm{cbo}}}(t)$ are of the form

$$
A_{m_{\mathrm{cbo}}}=A_{m}(t) \cos \left(\omega_{\mathrm{cbo}} t+\phi_{m}\right)
$$




\begin{tabular}{|c|c|c|c|c|}
\hline \multicolumn{5}{|c|}{ Low n-value Data Set } \\
\hline \hline & $\begin{array}{c}\text { main } \\
\text { cbo }\end{array}$ & $\begin{array}{c}\text { resid. } \\
g-2\end{array}$ & $\begin{array}{c}\text { lower cbo } \\
\text { sideband }\end{array}$ & $\begin{array}{c}\text { upper cbo } \\
\text { sideband }\end{array}$ \\
\hline \hline freq. (kHz) & 419.06 & 229.75 & 188.46 & 649.74 \\
\hline sigma (kHz) & 7.49 & 6.73 & 8.36 & 9.93 \\
\hline amp. (a.u.) & 22293. & 12224. & 6029.7 & 4739.6 \\
\hline \multicolumn{5}{|c|}{ High n-value Data Set } \\
\hline \hline & $\begin{array}{c}\text { main } \\
\text { cbo }\end{array}$ & $g$-2 & $\begin{array}{c}\text { lower cbo } \\
\text { sideband }\end{array}$ & $\begin{array}{c}\text { upper cbo } \\
\text { sideband }\end{array}$ \\
\hline \hline freq. (kHz) & 490.62 & 230.25 & 260.87 & 719.76 \\
\hline sigma (kHz) & 5.94 & 7.43 & 6.22 & 7.69 \\
\hline amp. (a.u.) & 25861. & 8355.1 & 6058.6 & 4512.0 \\
\hline
\end{tabular}

Table 4.1: Results of Gaussian fits to the four main peaks in the FFT spectrum of residuals to fits at late times.

where $m=N, A$, or $\phi$ and $A_{m}(t)$ is a monotonically decreasing function. Although vertical oscillations have a similar effect, simulation studies and data analysis have shown that the radial oscillations dominate this background. This is primarily because the vertical oscillations die out very quickly, with lifetimes of $\sim 20 \mu \mathrm{s}$.

The CBO envelope, $A_{f}(t)$, is well described by an exponential. Assuming $N_{\text {cbo }}, A_{\text {cbo }}$ and $\phi_{\text {cbo }}$ all have the same functional form, then there are 11 free parameters for the ratio method: three from the original ratio equation, three CBO amplitudes, three CBO phases, the CBO lifetime, and the CBO frequency. Note that there is no $N_{0}$ or $\tau$ when fitting a ratio. However, we can reduce the number of parameters by obtaining the CBO frequency and lifetime by other means.

To obtain the CBO frequency, we use the time spectrum of the residuals of a fit. A residual is the differences between the data and the fit function. The decay electron time spectrum is fit to the five parameter function at $\sim 180 \mu \mathrm{s}$ after injection. The residuals are then calculated for all times: $D\left(t_{i}\right)=N\left(t_{i}\right)-N_{i}$. This approach subtracts out the large $g-2$ signal and allows one to see the small backgrounds at early times after injection.

Figs. 4.21-4.22 show the results of Fast Fourier Transforms (FFTs) of the residuals of late-time fits for both the low-n and high-n data set. The top plots show the frequency range of $100-1000 \mathrm{kHz}$, in which we see quite clearly four peaks: the main CBO peak, the residual $g$-2 signal at $\simeq 229.1 \mathrm{kHz}$, and the "lower" and "upper" CBO sidebands $\left(f_{\text {cbo }}-f_{a}\right.$ and $f_{\text {cbo }}+f_{a}$ ).

Gaussian fits around the peaks were performed, the results of which are summarized in Table 4.1 (the results are also printed in the upper right corners of each plot). The top plots show the range of frequencies from 100 to $1000 \mathrm{kHz}$, and the fit results of the CBO peak are printed in the corner. The bottom plots show narrower frequency ranges to zoom in on the sideband frequencies, the left plot showing the lower CBO sideband and the right plot showing the upper CBO sideband.

We may further reduce the number of free parameters in our fit function by determining the CBO lifetime by again using the average energy vs. time. For this study we use use 

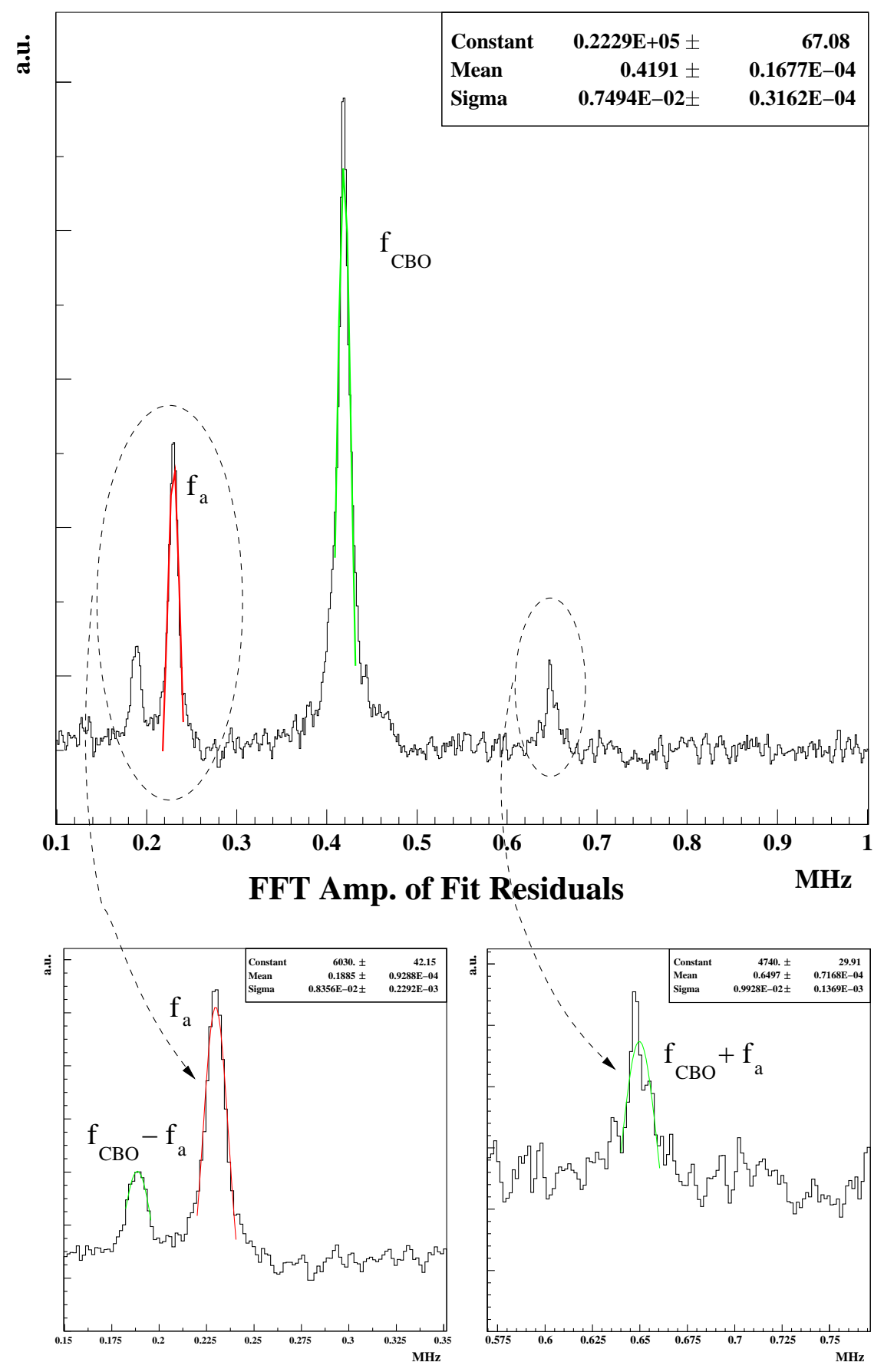

Figure 4.21: FFT of the residuals of a five parameter fit of the low-n electron decay time spectrum at $180 \mu \mathrm{s}$ after injection. The $f_{\mathrm{CBO}}$, residual $f_{a}$, and $f_{\mathrm{CBO}} \pm f_{a}$ peaks were fit to a Gaussian, the results of which are shown in Table 4.1. 

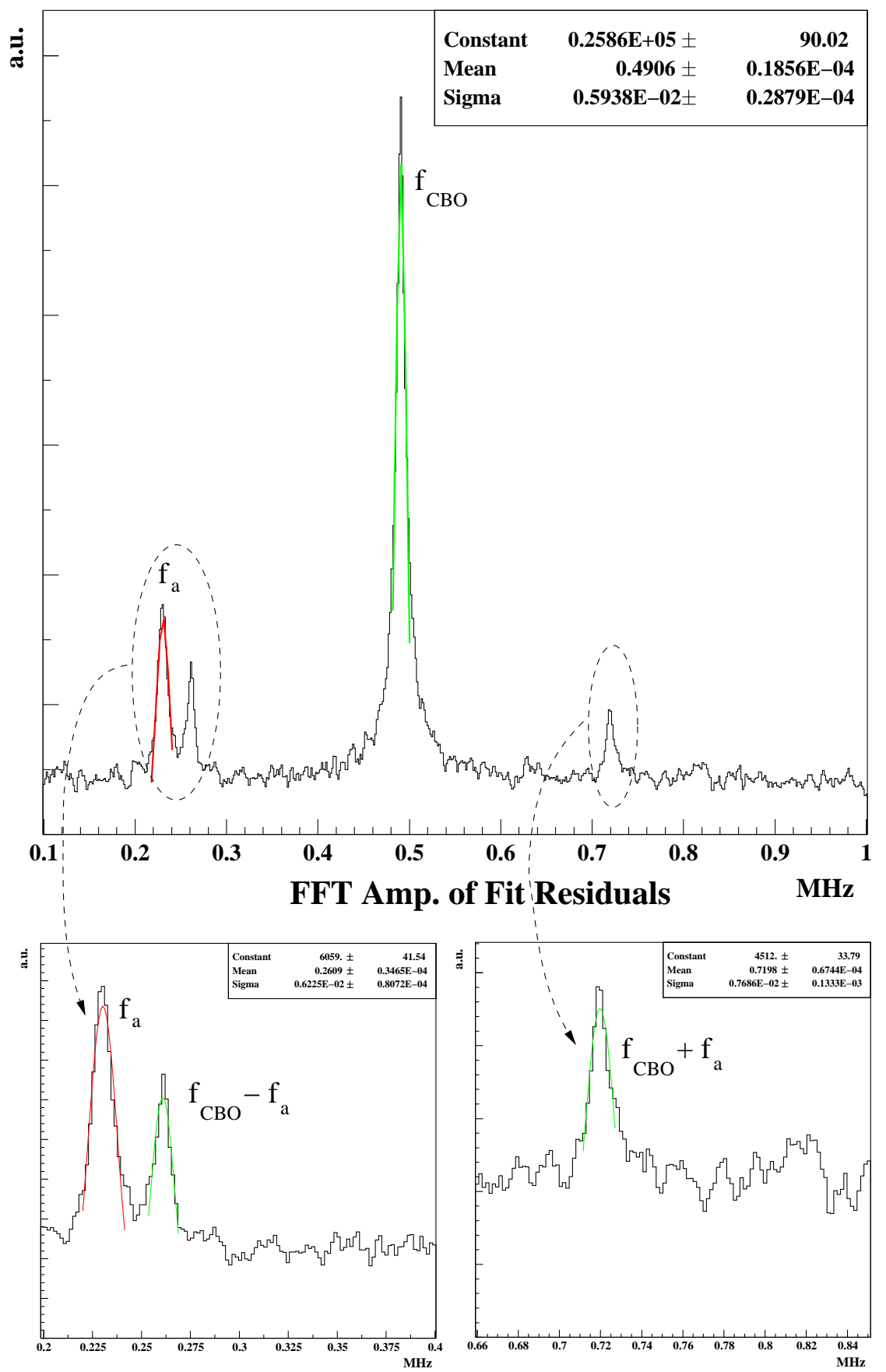

Figure 4.22: FFT of the residuals of a five parameter fit of the high-n electron decay time spectrum at $180 \mu \mathrm{s}$ after injection. The $f_{\mathrm{CBO}}$, residual $f_{a}$, and $f_{\mathrm{CBO}} \pm f_{a}$ peaks were fit to a Gaussian, the results of which are shown in Table 4.1. 

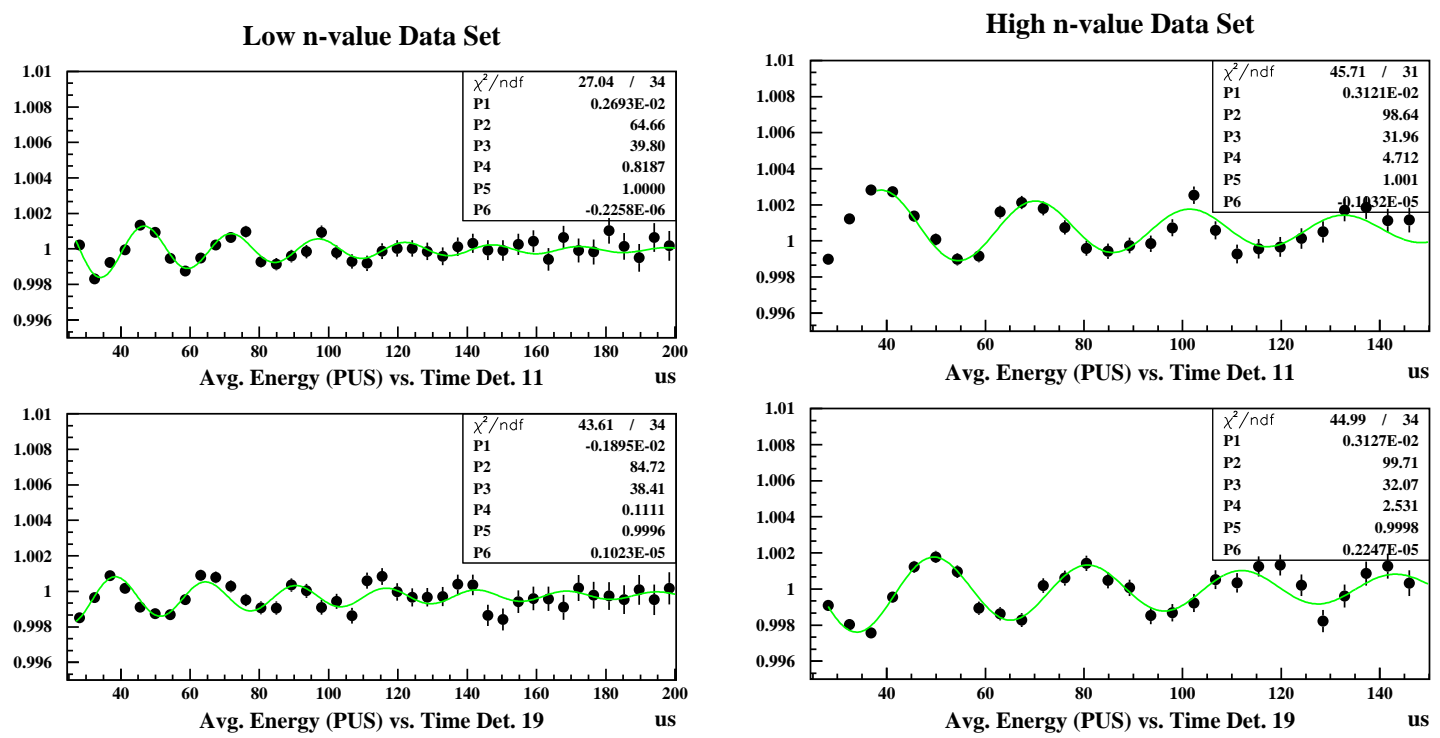

(a) Low n-value data set.

(b) High n-value data set.

Figure 4.23: Average energy vs. time where only decay electrons falling within the same $g$-2 phase are considered. The acceptance effect of CBO is very apparent, and the histograms are fit to Eq. 4.21 in order to extract the frequency and lifetime of the CBO.

time bin widths of $T_{a} / 8 \mu$ s instead of averaging the energy over one $g-2$ period. The energy distribution varies a great deal over a $g$ - 2 period, since at the peak of the $g$ - 2 period there are many more high-energy decay electrons than at the trough of the period. With no other energy-dependent backgrounds, the energy distribution should be the same every eighth time bin (always at the same $g$-2 phase). However, CBO acceptance effects are energydependent, and have a measurable effect on the average energy. A few examples of the CBO acceptance effect on the average energy are shown in Fig. 4.23, for detectors 11 and 19, for both data sets.

The oscillations are quite clear, and in fact are present in nearly all eight $g$ - 2 phases, not just those near the $g-2$ peak. Therefore the data for detectors 9-24 (except 20) were fit (in the range of $\sim 30$ to $\sim 200 \mu \mathrm{s}$ ) to the function

$$
\left\langle E_{T_{a} / 8}\right\rangle(t)=A e^{-t / \tau_{c}} \cos (\omega t+\phi)+c_{1} t+c_{0}
$$

where $A=p 1, \tau_{c}=p 2, \omega=\omega_{\text {cbo }}-\omega_{a}=p 3, \phi=p 4, c_{1}=p 5$ and $c_{0}=p 6$. We have assumed an exponential decay as the CBO envelope, and the $c_{1}$ and $c_{0}$ parameters of the fit function are present in order to account for actual gain. For detectors 9-24, a linear function is sufficient to describe the changes in average energy. Since there are eight $g-2$ phases and 15 detectors used in this study, a total of 120 fits were made. Cuts on the fit results were applied (reasonable values of fit $\chi^{2} /$ d.o.f. and reasonable values of fit parameters and errors on the fit parameters), and typically about $2 / 3$ of the fits were acceptable. 
Low n-value Data Set

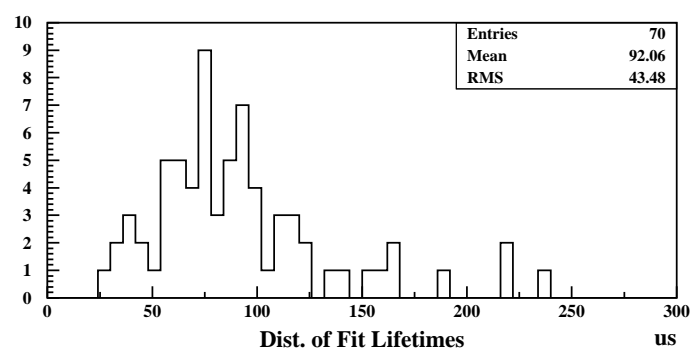

(a) Distributions of fit CBO lifetimes, low-n data.

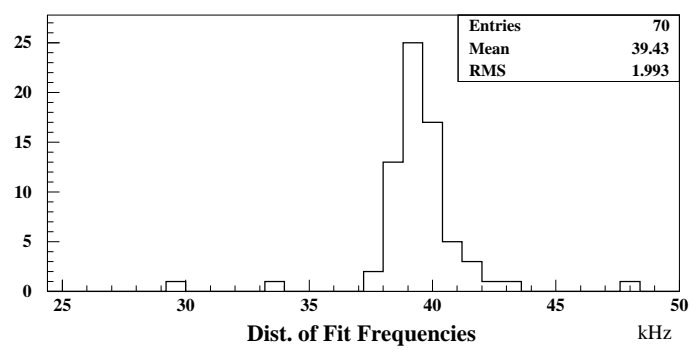

(c) Distribution of fit CBO frequencies, low-n data.

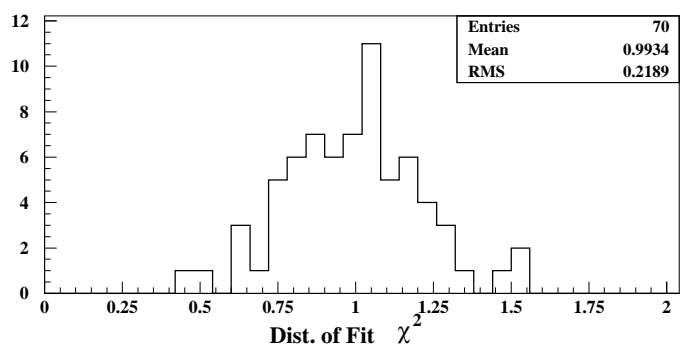

(e) Distribution of fit $\chi^{2} /$ d.o.f. , low-n data.
High n-value Data Set

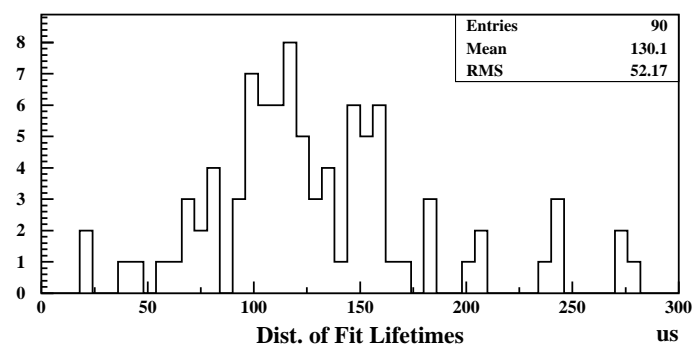

(b) Distributions of fit CBO lifetimes, high-n data.

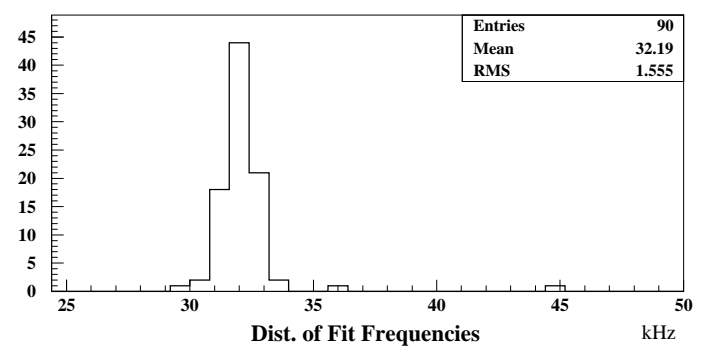

(d) Distribution of fit CBO frequencies, highn data.

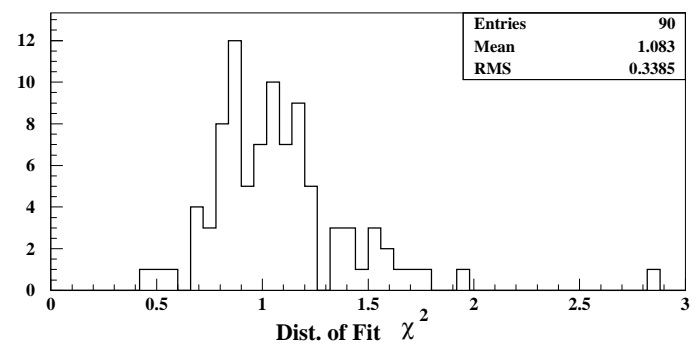

(f) Distribution of fit $\chi^{2} /$ d.o.f., high-n data.

Figure 4.24: Distributions of CBO parameters obtained from fits to $\left\langle E_{T_{a} / 8}\right\rangle$ vs. $t$ to Eq. 4.21. 


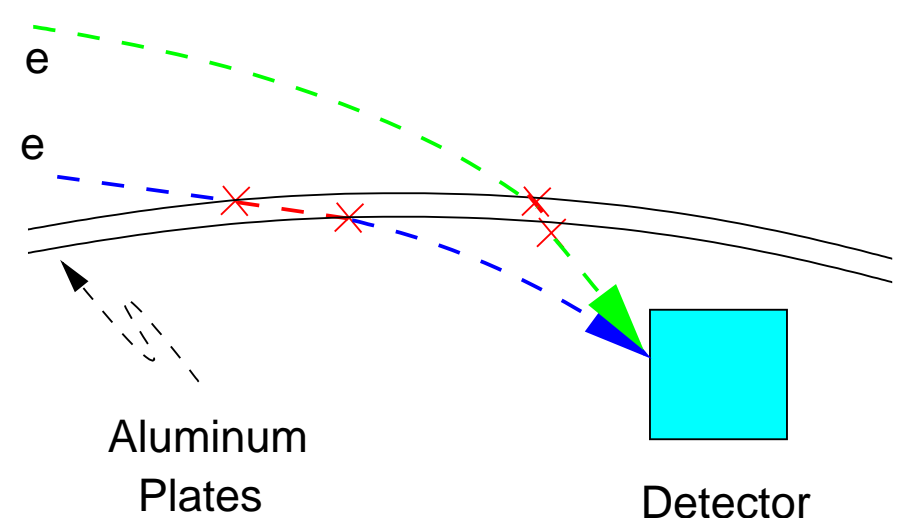

Figure 4.25: Effect of the kicker and quadrupole plates: electrons born at larger radii pass through less of the kicker and quadrupole plate material $(\mathrm{Al})$ than electrons born at smaller radii.

The distributions of the fitted CBO lifetimes and frequencies and fit $\chi^{2} /$ d.o.f. of the acceptable fits are shown in Fig. 4.24. The mean CBO frequencies, $f=\left|f_{c}-2 f_{a}\right|$, are found to be $f_{\text {cbo }}=418.7 \pm 0.2$ and $490.3 \pm 0.2 \mathrm{kHz}$ for the low and high n-value data sets respectively. These agree to within $2 \sigma$ with the CBO frequencies found above. The means of the distributions of the fit $\chi^{2}$ also agree with the expected value of 1.0 to within $2 \sigma$. Finally, the mean CBO lifetimes, which are fixed to their central values in the final ratio fits are $92.1 \pm 5.2$ and $130.1 \pm 5.5 \mu$ s for the low and high n-value data sets respectively.

\section{CBO vs. Detector}

There are two important features of the CBO spectra in the electromagnetic calorimeters that have important repercussions:

- The observed phase of the CBO oscillations varies from 0 to $2 \pi$ around the storage ring.

- Some detectors are more sensitive to CBO oscillations than others.

The first point is simply the result of observing the electron decay spectrum at different locations in the ring. This implies that adding data from all detectors together would cancel the effect of CBO. However, this would only work if all detectors had the same acceptance. Unfortunately, this is not the case.

Different detector have different acceptances because of objects, typically aluminum plates, inside the storage ring just upstream of the detector. For example, a "shadow" of the kicker and quadrupole plates is observed in the detectors located nearby. When passing through the plates, the decay electrons shower and lose energy. Since the beam is moving in and out radially, electrons born at larger radii pass through less aluminum than electrons born at smaller radii (see Fig. 4.25), resulting in a time-dependent distortion of the observed energy spectrum. We therefore expect to see larger CBO effects in those detectors near the muon kickers and near the electrostatic quadrupole regions. In fact, since the kicker plates 
are thicker and taller than the quadrupole plates, the effect is even larger for those detectors near the kicker plates.

\subsubsection{Fit Procedure}

To fit the ratio time spectra, the $\chi^{2}$ of the ratio function,

$$
\chi^{2}=\sum_{i} \frac{\left(r\left(t_{i}\right)-r_{i}\right)^{2}}{\sigma_{i}^{2}}
$$

is minimized. $t_{i}$ is the central value of the time bin, $r\left(t_{i}\right)$ is the calculated value of the ratio at time $t_{i}, r_{i}$ is the measured value of the ratio in the $i^{t h}$ bin, and $\sigma_{i}$ is the uncertainty on $r_{i}$. Note that since we have taken a ratio, $\sigma_{i}$ is not the $\sqrt{N_{i}}$ that one would typically use in a fit to the measured number of counts. Appendix B.4 gives a full derivation of $\sigma_{i}$, which is of the form

$$
\sigma_{i}^{2} \simeq \frac{\left(1-r_{i}^{2}\right)}{U_{i}+V_{i}}
$$

where $U_{i}+V_{i}$ is the sum of the shifted and unshifted data at time $t_{i}$.

The $\chi^{2}$ is minimized using the CERNLIB package MINUIT. MINUIT is designed to find the minimum value of a multi-parameter function [92], and accepts a variety of commands to specify the fit procedure. In this analysis, the following commands are given to MINUIT:

(1) MIGRAD

(2) IMPROVE

(3) MINOS

(4) MIGRAD

The command MIGRAD minimizes the function with "the most efficient and complete single method" [92]. IMPROVE searches "for additional distinct local minima" [92], and MINOS performs a very reliable error analysis calculation which takes "account of non-linearities in the problem as well as parameter correlations ..." [92]. We use the information from the previous calls in the last call to MIGRAD to obtain the final values of the free parameters in the fit, although this has little, if any, effect on the final fit values.

\subsubsection{Fit Results}

The data from the 2001 run were fit to various ratio functions, ranging from the simple three parameter function to a nine parameter function. The following sections discuss the results from four fit functions, each function an extension of the previous one. We note that data from detector 20 are never used. Also, data from the 23 detectors were summed together and fit; for ease of reference, the summed data is referred to as detector 25. It is also useful to compare fit results of data summed over different halves of the full detector set. Therefore, detector 26 represents the summed data from detectors 1-12, and detector 27 represents the summed data from detectors 13-24, detector 20 excluded. 
Low-n Data Set, Three Par. Fit
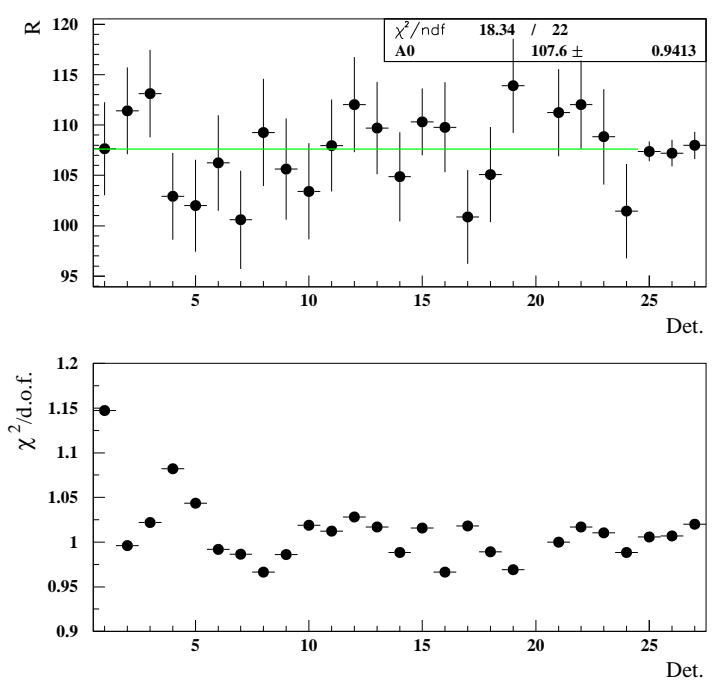

(a) Low-n data set.
High-n Data Set, Three Par. Fit
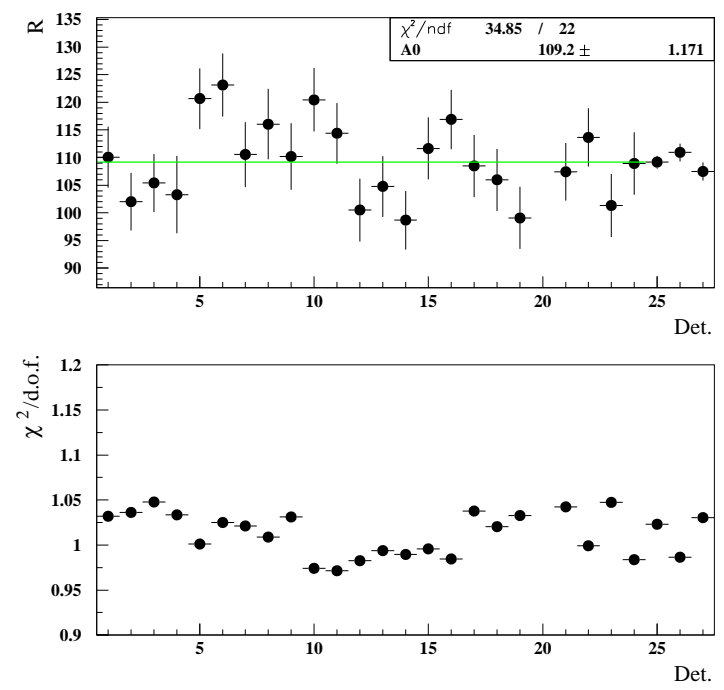

(b) High-n data set.

Figure 4.26: Top: $R$ vs. Detector for the three parameter fit. Bottom: Fit $\chi^{2} /$ d.o.f. for each detector for the three parameter fit. All points are taken at $\mathrm{t}=32.5 \mu \mathrm{s}$.

\section{Three Parameter Fit}

The first function the data were fit to was the simple three parameter ratio function,

$$
r_{3}(t)=\boldsymbol{A} \cos \left(2 \pi f_{a}\left(1-\left(\boldsymbol{R}-R_{o f f}\right) \times 10^{-6}\right) t+\boldsymbol{\phi}_{\boldsymbol{a}}\right)-C_{1}
$$

where the bold parameters are free in the fit, $f_{a}=229.1 \mathrm{kHz}$ and $C_{1}=2.874 \times 10^{-4} . R_{\text {off }}$ is an offset equal to $-7.80 \mathrm{ppm}$, originally known only to the $\omega_{a}$ analyzers and used to ensure a blind analysis of $a_{\mu}$ (the $\omega_{p}$ analyzers used a different offset known only to themselves until the final value of $a_{\mu}$ was calculated). Fit values of $R$ and fit $\chi^{2} /$ d.o.f. at a fit start time of $32.5 \mu \mathrm{s}$ are plotted as a function of detector number in Fig. 4.26. Results for the low-n data set are on the left, the high-n results are on the right. The first 24 detectors in the $R$ vs. detector plot are fit to a constant, which gives the average $R$ value over the detectors. The values for detector 20 are ignored in the fit. The resulting average $R$ values, for the low- and high-n data sets respectively, are $107.6 \pm 0.94 \mathrm{ppm}$ and $109.2 \pm 1.17 \mathrm{ppm}$, where the error is obtained from the fit to a constant.

The fit start time dependence of $R, A$ and fit $\chi^{2} /$ d.o.f. is shown in Fig. $4.27^{2}$. The curved lines drawn on some plots are the correlated difference error bands. The top plots of Fig. 4.27 shows $R$ vs. as a function of fit start time for the detectors 25,26 and 27 . For the low-n data set, we see that from about 30 to $100 \mu \mathrm{s}$, the $R$-values for both halves of the ring

\footnotetext{
${ }^{2}$ Since $\phi_{a}$ is highly correlated to $R$, its time dependence tracks almost exactly with $R$ 's; we learn little from plots of $\phi_{a}$ vs. fit start time.
} 

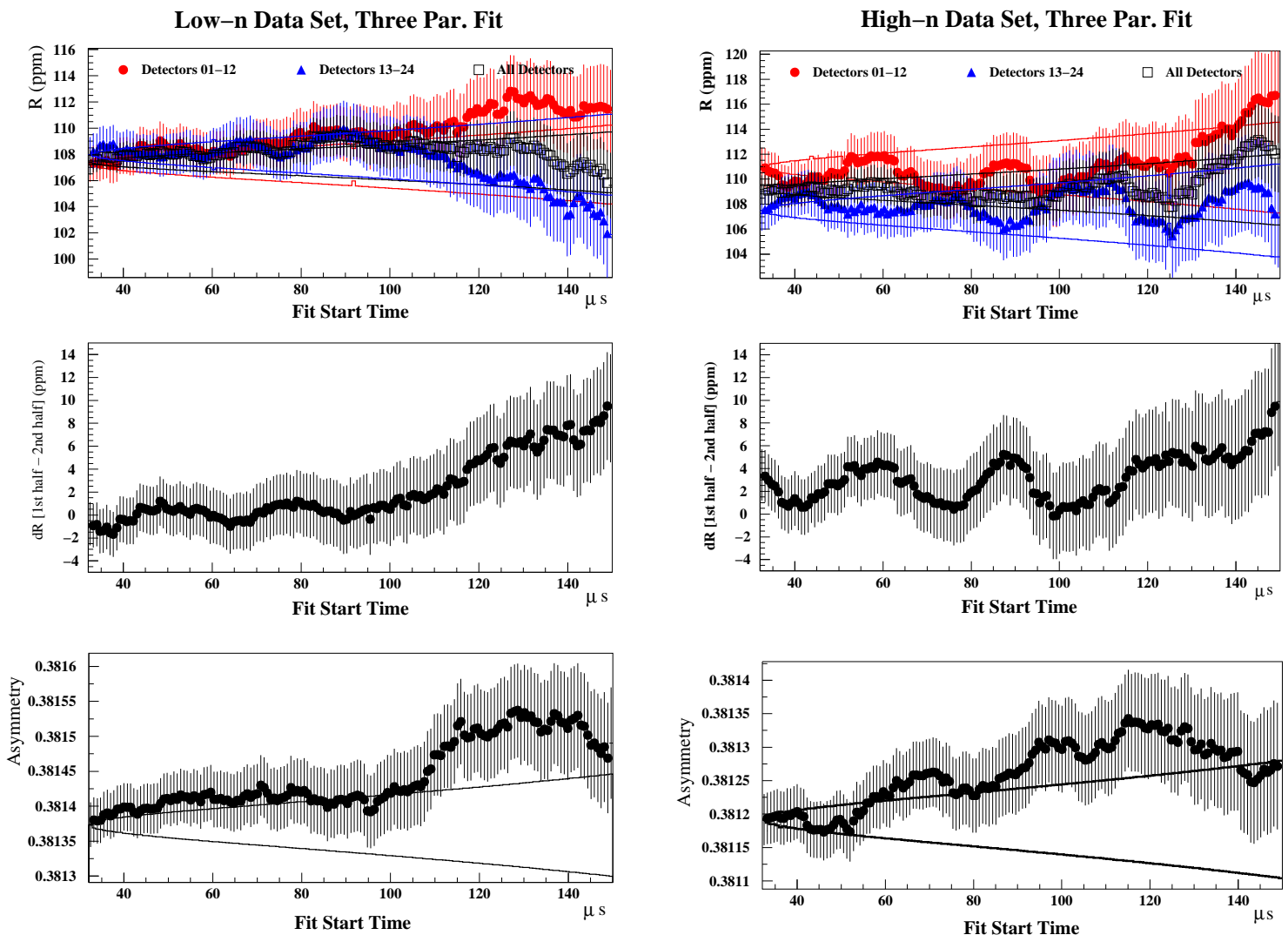

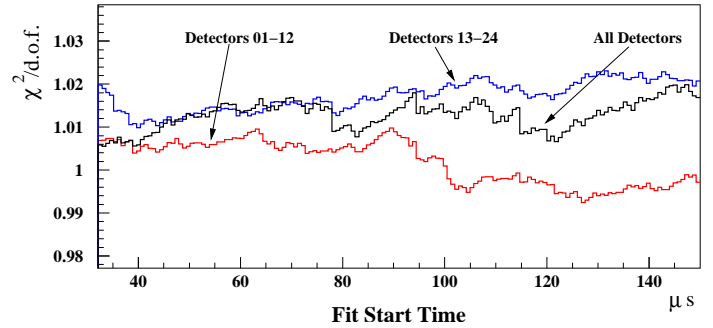

(a) Low-n data set.

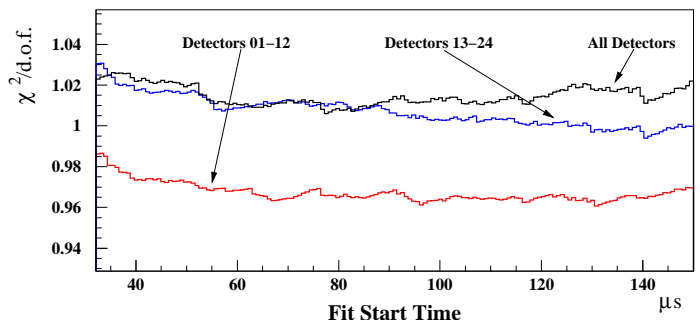

(b) High-n data set.

Figure 4.27: From top to bottom: 1 . $R$ vs. fit start time for the three parameter fit for different combinations of detectors: 1-12 (red circles), 13-24 (blue triangles), and 1-24 (black squares). 2. Difference of $\mathrm{R}$ vs. fit start time plots between first and second halves of the ring. 3. Asymmetry vs. fit start time for detector 25. 4. $\chi^{2} /$ d.o.f. vs. fit start time for different combinations of detectors. 
track quite well. The second plot from the top shows the difference between the $R$ vs. fit start time plots for detectors 26 and 27. Although the fit $R$-values of the two halves of the ring do seem to diverge around $100 \mu \mathrm{s}$, they do not diverge by more than $2 \sigma$. Furthermore, studies have shown that they later converge around $200 \mu \mathrm{s}$. Therefore, this is most likely a statistical effect.

However, for the high-n data set, there is quite a large difference $(\sim 3 \pm 2 \mathrm{ppm}$ at $\sim 33$ $\mu \mathrm{s})$ in the $R$-values for the two halves of the ring at all fit start times. By fitting various subsets of the high-n data set and looking at the differences between the fit $\mathrm{R}$ values of the first and second halves of the ring, Peter Shagin has demonstrated that this $3 \mathrm{ppm}$ difference is very likely a statistical effect.

We also see oscillations of roughly $30 \mathrm{kHz}$ in the differences of the half-ring $R$ vs. fit start time plots, in both data sets. These oscillations, often referred to as the "half-ring effect", are related to $\mathrm{CBO}$ and the frequency is the difference $f_{\mathrm{CBO}}-2 f_{a}$.

The plots second from the bottom in Fig. 4.27 show the fit start time dependency of the asymmetry for detector 25 . The asymmetry appears to drift to a slightly larger value at late start times, which is possibly an effect of low-energy pileup. At early times, the low-energy pileup has a tendency to lower the average asymmetry (low-energy pulses have a small, negative asymmetry), and at later times the amount of pileup diminishes.

The bottom plots in Fig. 4.27 show the $\chi^{2} /$ d.o.f. for detectors 25-27. We note that for Gaussian statistics and $N$ degrees of freedom, $\chi^{2} /$ d.o.f. $=1 \pm \sqrt{2 / \mathrm{N}}$. Since the fits have

approximately 4400 degrees of freedom (time bins), the $\chi^{2} /$ d.o.f. should be $1 \pm 0.024$. The values of the fit $\chi^{2} /$ d.o.f. are therefore acceptable.

\section{Five Parameter Ratio Fit}

We now extend the fit function to include the main CBO background term, the modulation of $N: N_{\text {cbo }}(t)$. Our procedure parallels the CBO extensions to the functions used to fit the decay positron time spectra from the 1999 data set. Although one could use a parameterization for the ratio as described in Appendix B.3, we found that, in practice, fits to the data converged better by calculating the explicit ratio function:

$$
r_{5}(t)=\frac{2 f_{0}(t)-f_{+}(t)-f_{-}(t)}{2 f_{0}(t)+f_{+}(t)+f_{-}(t)}
$$

where

$$
f_{0}(t)=\left(1+e^{-t / \tau_{\mathrm{CBO}}} \boldsymbol{A}_{\boldsymbol{N}_{\mathrm{CBO}}} \cos \left(\omega_{\mathrm{CBO}} t+\phi_{\mathrm{CBO}}\right)\right)\left(1+\boldsymbol{A} \cos \left(\boldsymbol{\omega}_{\boldsymbol{a}} t+\boldsymbol{\phi}_{\boldsymbol{a}}\right)\right)
$$

and

$$
f_{ \pm}(t)=\left(1+e^{-t^{\prime} / \tau_{\mathrm{CBO}}} \boldsymbol{A}_{\boldsymbol{N}_{\mathbf{C B O}}} \cos \left(\omega_{\mathrm{CBO}} t^{\prime}+\phi_{\mathrm{CBO}}\right)\right) e^{\mp T /(2 \tau)}\left(1+\boldsymbol{A} \cos \left(\boldsymbol{\omega}_{\boldsymbol{a}} t^{\prime}+\boldsymbol{\phi}_{\boldsymbol{a}}\right)\right)
$$

where $t^{\prime}$ is the shifted time

$$
\begin{gathered}
t^{\prime}=t \pm T / 2, \\
\boldsymbol{\omega}_{\boldsymbol{a}}=2 \pi f_{a}\left(1-\left(\boldsymbol{R}-R_{o f f}\right) \times 10^{-6}\right),
\end{gathered}
$$


Low-n Data Set, Five Par. Fit
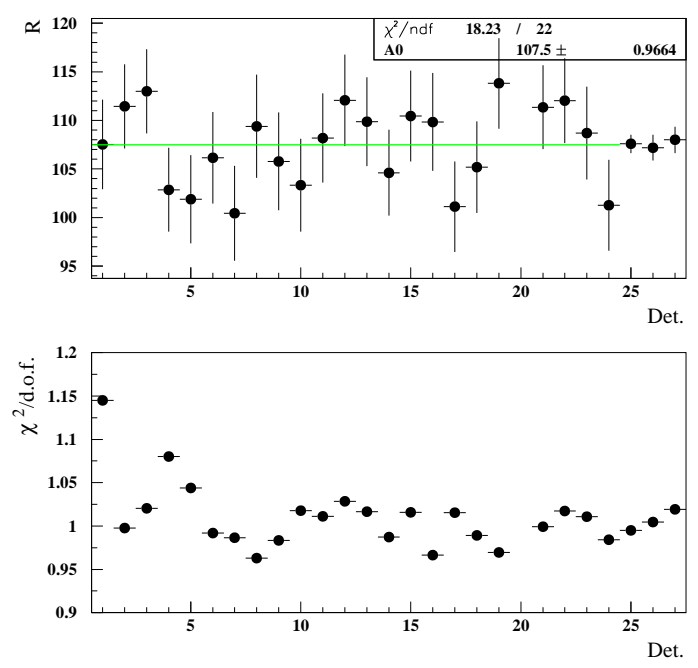

(a) Low-n data set.
High-n Data Set, Five Par. Fit
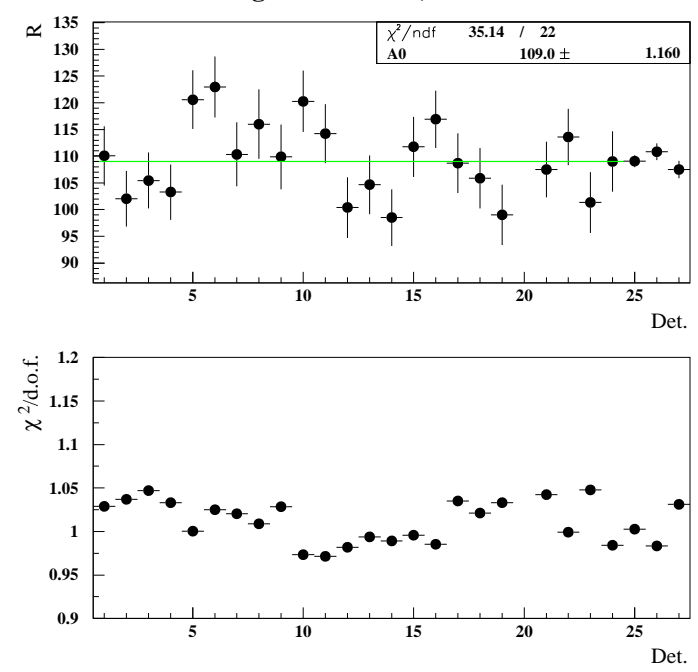

(b) High-n data set.

Figure 4.28: Top: R vs. Detector for the five parameter fit. Bottom: Fit $\chi^{2} /$ d.o.f. for each detector for the five parameter fit. All points are taken at $\mathrm{t}=32.5 \mu \mathrm{s}$.

$\tau$ is the dilated muon lifetime fixed to $64.4 \mu \mathrm{s}, \boldsymbol{A}_{N_{\text {CBO }}}$ is the amplitude of the CBO effect on $N$, and $\phi_{\mathrm{CBO}}$ is the $\mathrm{CBO}$ phase. Note that the $N_{0}$ and $\exp (-t / \tau)$ terms have been divided out already. The CBO frequencies and lifetimes were fixed to the values determined in Section 4.3.1: $419.1 \mathrm{kHz}$ and $92.1 \mu \mathrm{s}$ for the low-n data set and $490.6 \mathrm{kHz}$ and $130.1 \mu \mathrm{s}$ for the high-n data set.

Fig. 4.28 shows $R$ vs. detector for the two data sets. The fitted values for average $R$ at $32.5 \mu$ s are very consistent with those obtained from the three parameter fit. In fact, comparing all fit results in Fig. 4.29 of the five parameter fits to those of the three parameter fits (Fig. 4.27, we see no significant difference between the two.

$\mathrm{CBO}$ amplitudes and phases vs. detector are shown in Fig. 4.30, where the data points are taken at a fit start time of $32.5 \mu \mathrm{s}$. As expected, the CBO phase varies almost linearly as a function of detector number, covering a range of $2 \pi$ radians around the ring. Deviations from a straight line arise from statistical fluctuations and differences in detector acceptance.

As discussed in Section 4.3.1, it is expected that the observed CBO amplitude be larger for detectors near the kicker modules (detectors 7, 8, and 9). This has been verified by direct fits of the decay electron time spectrum [93] as well as from simulation. However, in fits to the ratio we see only a slightly larger $\mathrm{CBO}$ amplitude in some of the detectors near the kickers; in fact, one could well argue that most of the fit values agree within errors. The reason that larger $\mathrm{CBO}$ amplitudes are not observed in the ratio fits for the detectors near the kicker is that the ratio is less sensitive to the main CBO term (see Appendix B.3). Indeed, it is for this reason that the half-ring oscillations are still present after fitting the ratio with the five parameter function. We therefore conclude that the function used to fit 

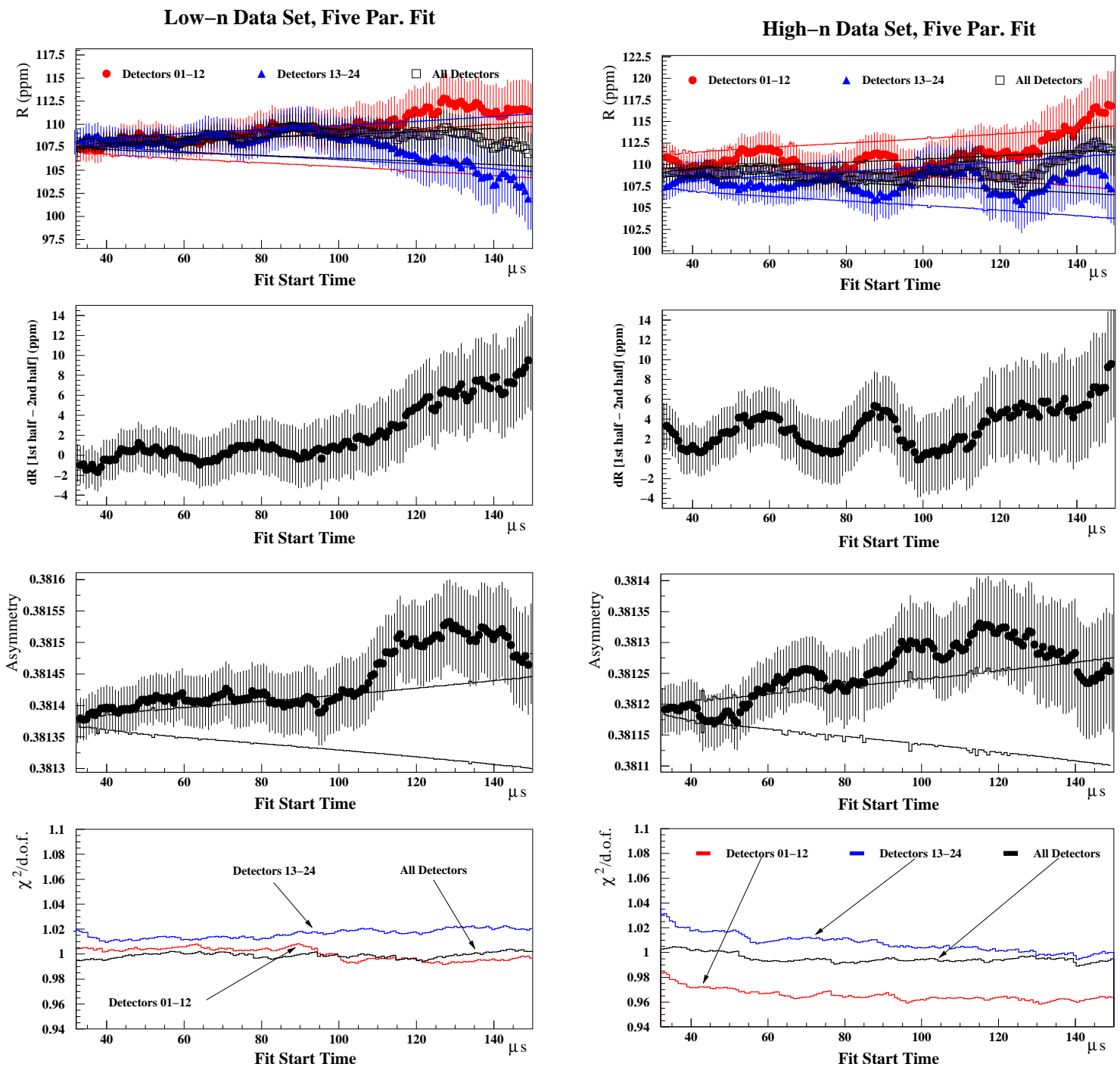

(a) Low-n data set.

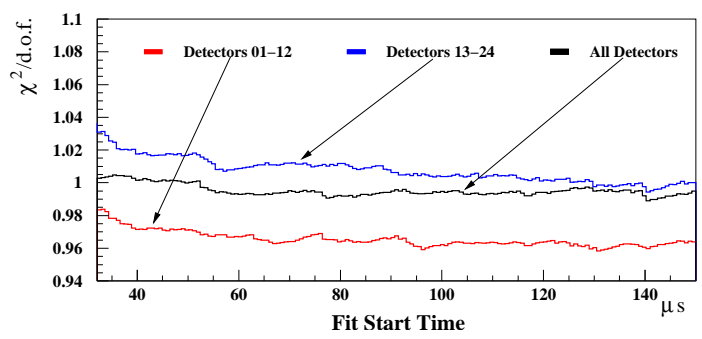

(b) High-n data set.

Figure 4.29: From top to bottom: 1. $R$ vs. fit start time for the five parameter fit for different combinations of detectors: 1-12 (red circles), 13-24 (blue triangles), and 1-24 (black squares). 2. Difference of $\mathrm{R}$ vs. fit start time plots between first and second halves of the ring. 3. Asymmetry vs. fit start time for detector 25. 4. $\chi^{2} /$ d.o.f. vs. fit start time for different combinations of detectors. 
Low-n Data Set, Five Par. Fit
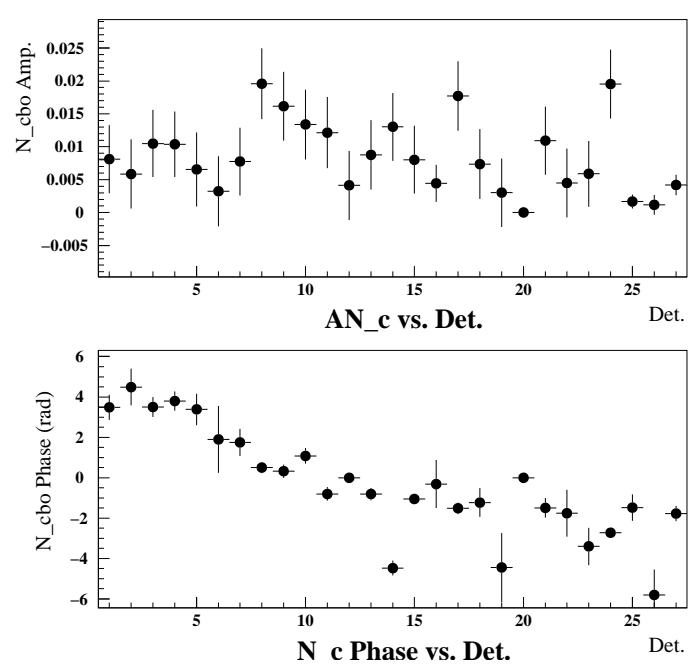

(a) Low-n data set.
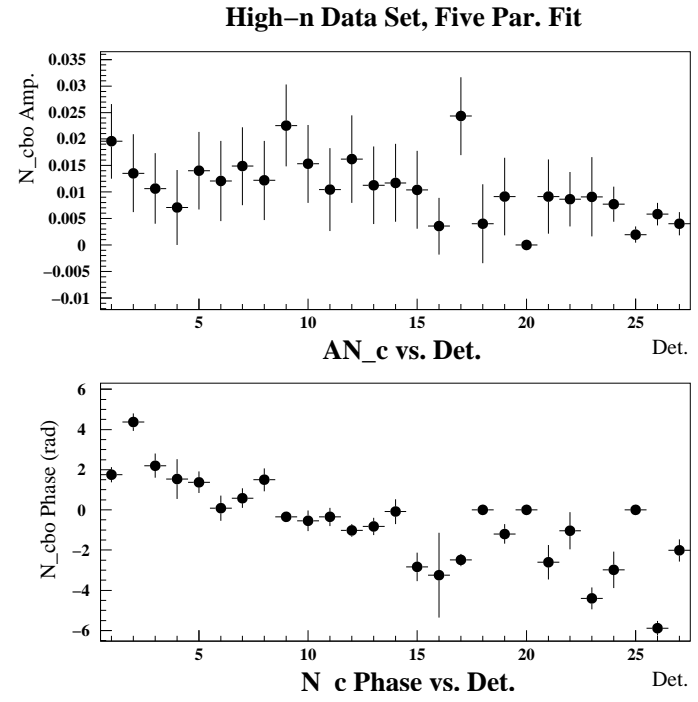

(b) High-n data set.

Figure 4.30: CBO amplitude (top) and phase (bottom) vs. detector for the five parameter ratio fit.

the data is still inadequate.

\section{Seven Parameter Ratio Fit}

We once gain extend our fit function to include another CBO term, in this case, $A_{\text {cbo }}(t)$. The data were fit to the function:

$$
r_{7}(t)=\frac{2 f_{0}(t)-f_{+}(t)-f_{-}(t)}{2 f_{0}(t)+f_{+}(t)+f_{-}(t)}
$$

where

$$
f_{0}(t)=\left(1+\boldsymbol{A}_{\boldsymbol{N}_{\mathrm{CBO}}}(t)\right)\left(1+\boldsymbol{A}\left(1+\boldsymbol{A}_{\boldsymbol{A}_{\mathrm{CBO}}}(t)\right) \cos \left(\boldsymbol{\omega}_{\boldsymbol{a}} t+\boldsymbol{\phi}_{\boldsymbol{a}}\right)\right)
$$

and

$$
f_{ \pm}(t)=\left(1+\boldsymbol{A}_{\boldsymbol{N}_{\mathrm{CBO}}}\left(t^{\prime}\right)\right) e^{\mp T /(2 \tau)}\left(1+\boldsymbol{A}\left(1+\boldsymbol{A}_{\boldsymbol{A}_{\mathrm{CBO}}}\left(t^{\prime}\right)\right) \cos \left(\boldsymbol{\omega}_{\boldsymbol{a}} t^{\prime}+\boldsymbol{\phi}_{\boldsymbol{a}}\right)\right)
$$

where $t^{\prime}$ is the shifted time

$$
\begin{gathered}
t^{\prime}=t \pm T / 2, \\
\boldsymbol{\omega}_{\boldsymbol{a}}=2 \pi f_{a}\left(1-\left(\boldsymbol{R}-R_{o f f}\right) \times 10^{-6}\right),
\end{gathered}
$$

as before, $\tau$ is the dilated muon lifetime fixed to $64.4 \mu \mathrm{s}$,

$$
\boldsymbol{A}_{\boldsymbol{N}_{\mathrm{CBO}}}(t)=\boldsymbol{A}_{N_{\mathrm{CBO}}} e^{-t / \tau_{\mathrm{CBO}}} \cos \left(\omega_{\mathrm{CBO}} t+\phi_{N_{\mathrm{CBO}}}\right)
$$


Low-n Data Set, Seven Par. Fit
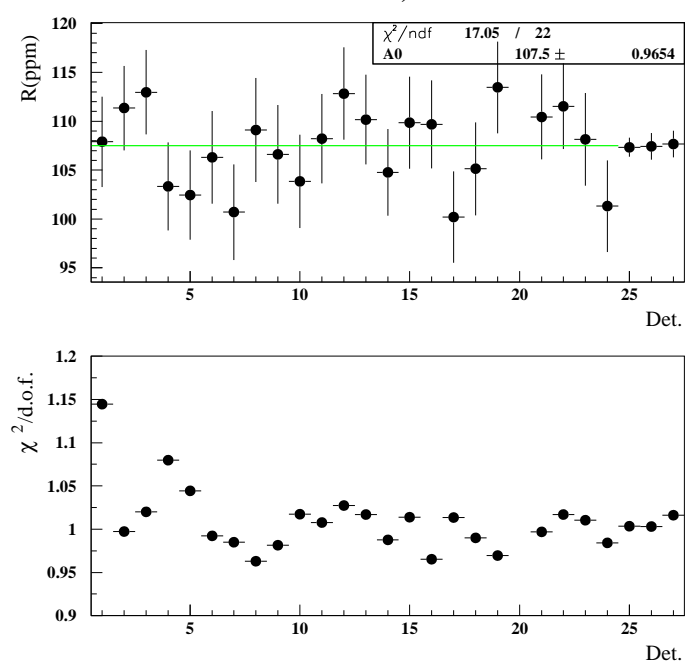

(a) Low-n data set.
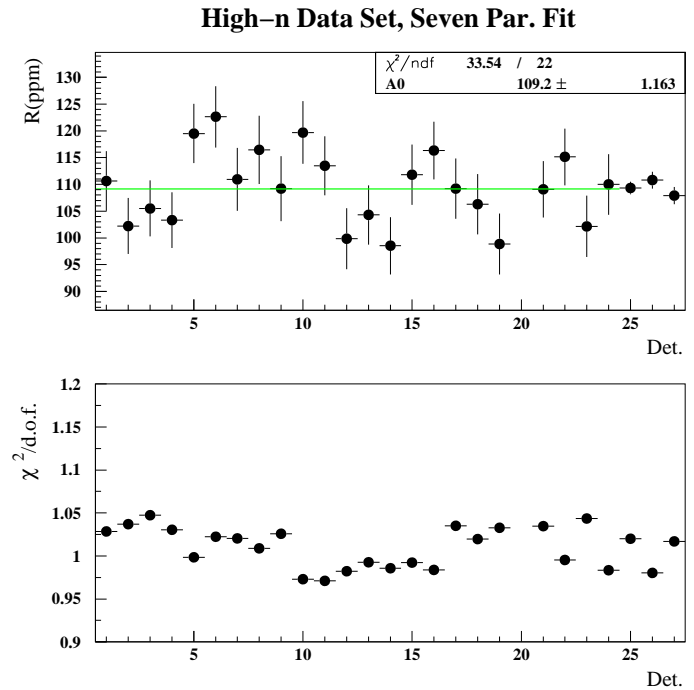

(b) High-n data set.

Figure 4.31: R vs. Detector for the seven parameter ratio fit. Left: Low-n data set. Right: High-n data set. All points are taken at $\mathrm{t}=32.5 \mu \mathrm{s}$.

is the amplitude of the CBO effect on $N$, and

$$
\boldsymbol{A}_{\boldsymbol{A}_{\mathrm{CBO}}}(t)=\boldsymbol{A}_{\boldsymbol{A}_{\mathrm{CBO}}} e^{-t / \tau_{\mathrm{CBO}}} \cos \left(\omega_{\mathrm{CBO}} t+\phi_{\boldsymbol{A}_{\mathrm{CBO}}}\right)
$$

is the amplitude of the CBO effect on $\boldsymbol{A}$. The CBO frequencies and lifetimes are fixed to the same values used in the five-parameter ratio fit.

The fit results for $\mathrm{R}, \chi^{2}, N_{\mathrm{CBO}}$ and $A_{\mathrm{CBO}}$ are shown in Figs. 4.31-4.34. We see a dramatic improvement in the fits with regards to $\mathrm{CBO}$ compared to the three and five parameter fits. The amplitude of the half-ring effect is greatly diminished (see Fig. 4.31). And again, as expected, $\phi_{N_{\mathrm{CBO}}}$ and $\phi_{A_{\mathrm{CBO}}}$ both traverse $2 \pi$ radians around the circumference of the storage ring.

\section{Nine Parameter Ratio Fit}

Although the seven-parameter ratio function eliminates the half-ring effect, we expect CBO to influence the $g$-2 phase, as discussed above. To include the CBO modulation of the $g-2$ phase, we use a nine parameter fit function:

$$
r_{9}(t)=\frac{2 f_{0}(t)-f_{+}(t)-f_{-}(t)}{2 f_{0}(t)+f_{+}(t)+f_{-}(t)}
$$

where

$$
f_{0}(t)=\left(1+\boldsymbol{A}_{\boldsymbol{N}_{\mathrm{CBO}}}(t)\right)\left(1+\boldsymbol{A}\left(1+\boldsymbol{A}_{\boldsymbol{A}_{\mathrm{CBO}}}(t)\right) \cos \left(\boldsymbol{\omega}_{\boldsymbol{a}} t+\phi_{\boldsymbol{a}}\left(1+\boldsymbol{A}_{\boldsymbol{\phi}_{\mathrm{CBO}}}(t)\right)\right)\right)
$$


Low-n Data Set, Seven Par. Fit
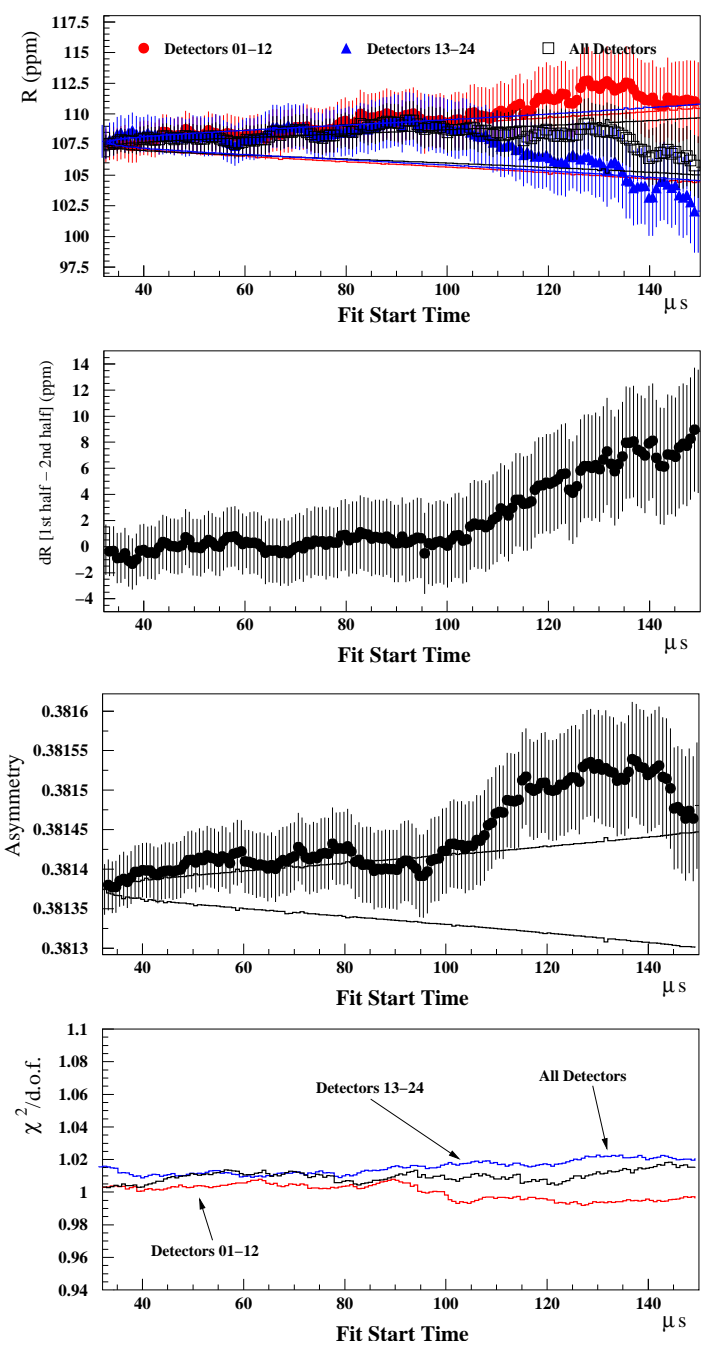

(a) Low-n data set.
High n-value, 7 Par. Fit
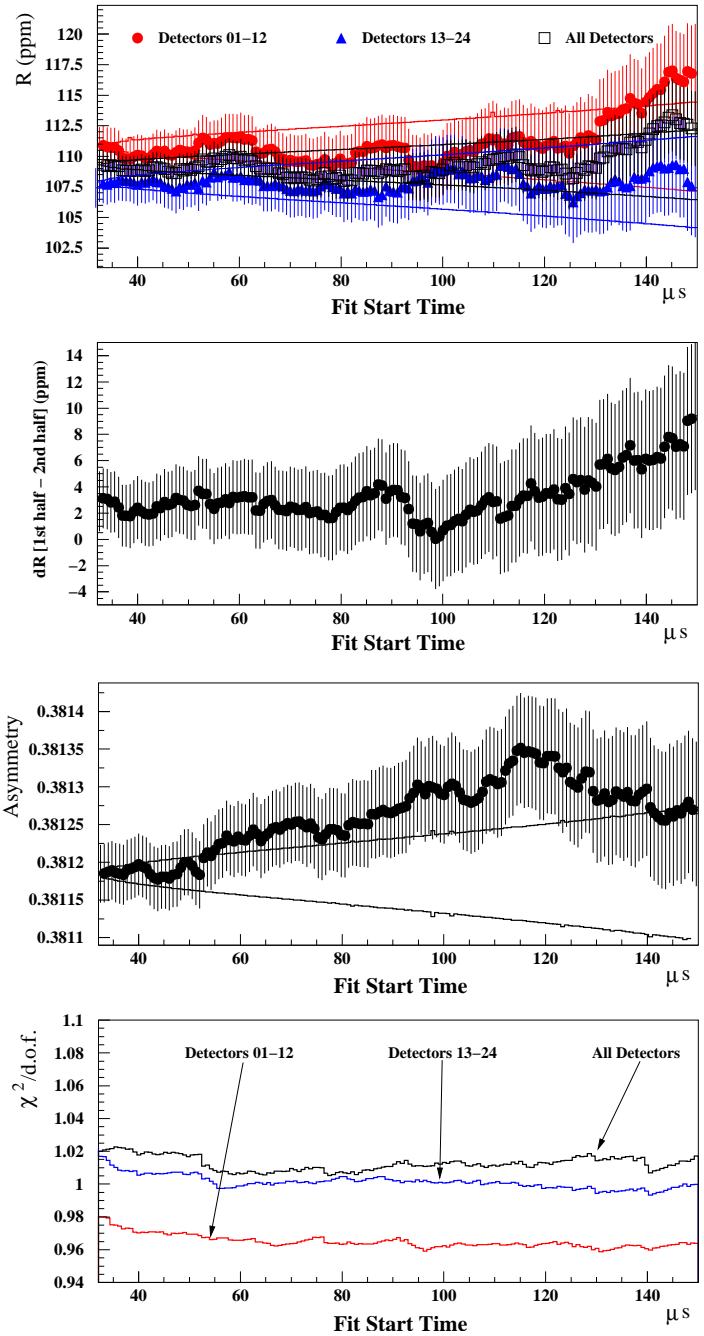

(b) High-n data set.

Figure 4.32: From top to bottom: 1 . $R$ vs. fit start time for the seven parameter fit for different combinations of detectors: 1-12 (red circles), 13-24 (blue triangles), and 1-24 (black squares). 2. Difference of $\mathrm{R}$ vs. fit start time plots between first and second halves of the ring. 3. Asymmetry vs. fit start time for detector 25. 4. $\chi^{2} /$ d.o.f. vs. fit start time for different combinations of detectors. 

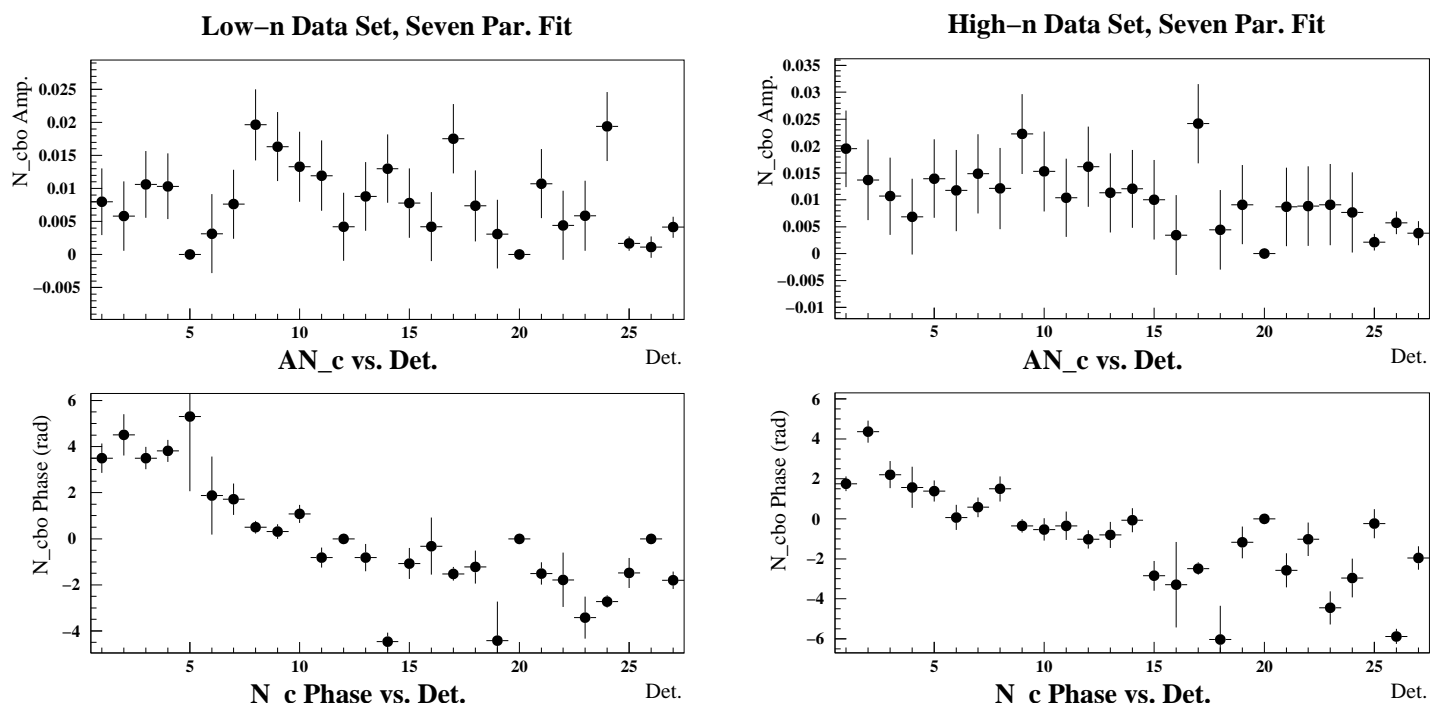

(a) Low-n data set.

(b) High-n data set.

Figure 4.33: $N_{\mathrm{CBO}}$ amplitudes and phases vs. Detector for the seven parameter ratio fit.
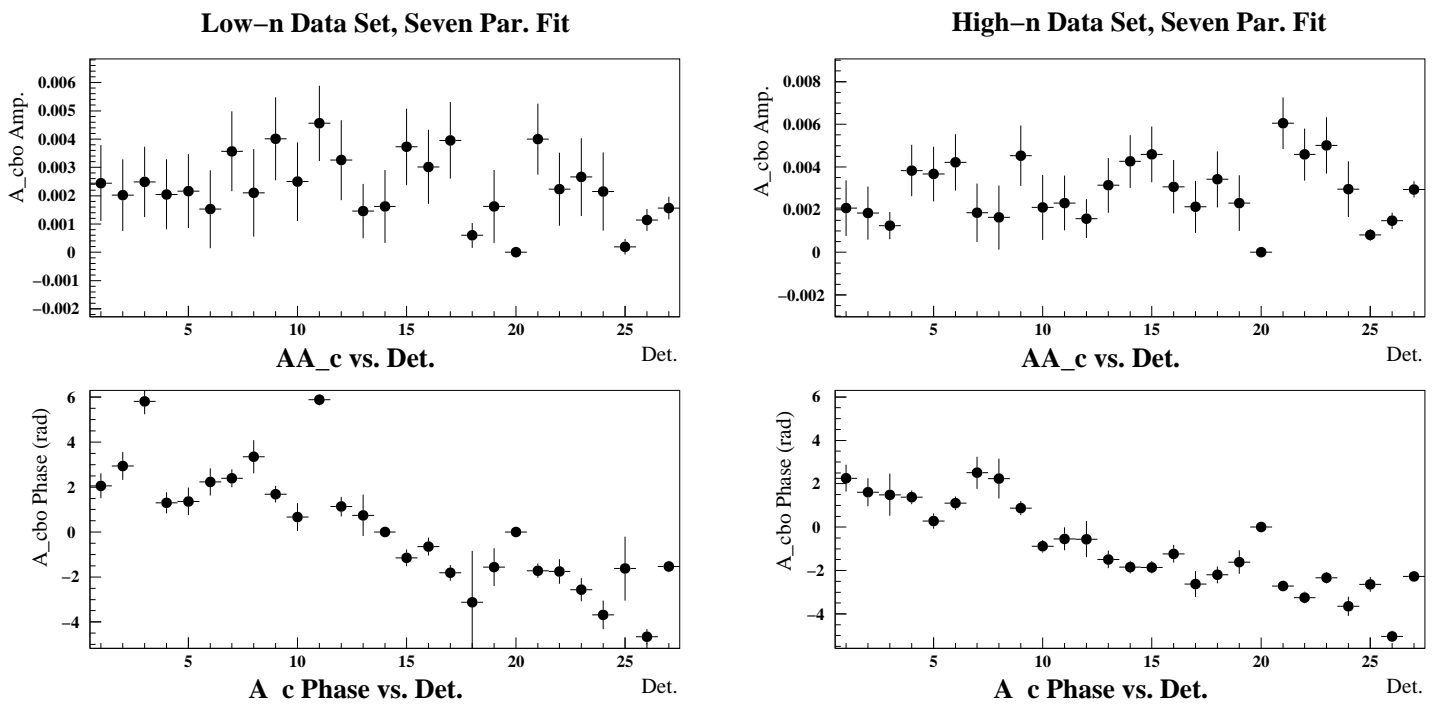

(a) Low-n data set.

(b) High-n data set.

Figure 4.34: $A_{\mathrm{CBO}}$ term amplitudes and phases vs. Detector for the seven parameter ratio fit. 
Low-n Data Set, Nine Par. Fit
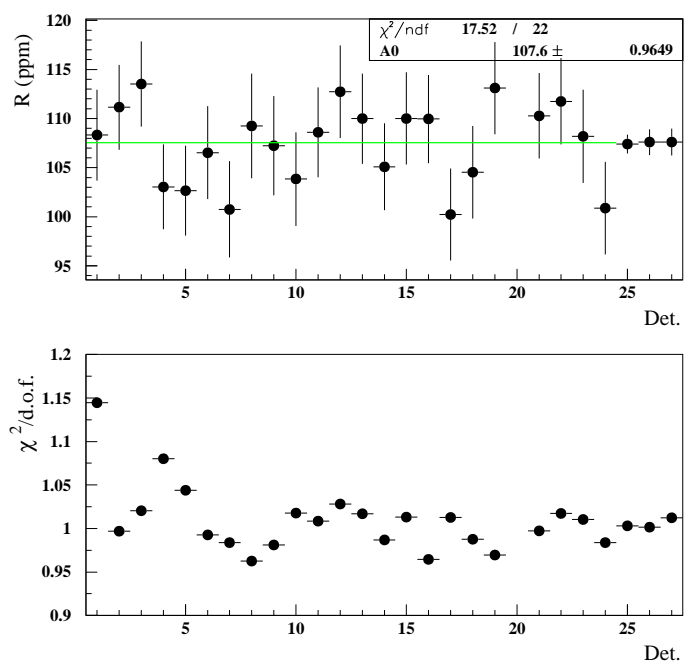

(a) Low-n data set.
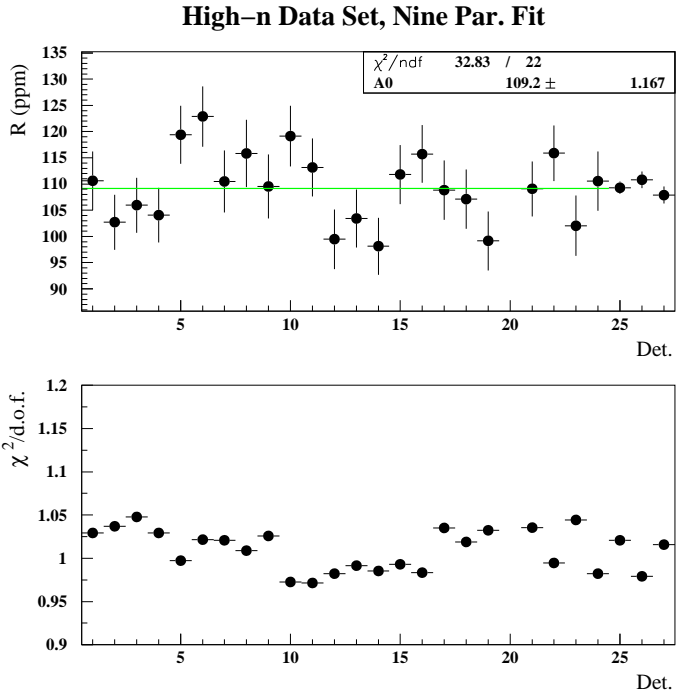

(b) High-n data set.

Figure 4.35: R vs. Detector for the nine parameter fit. All points are taken at $\mathrm{t}=32.5 \mu \mathrm{s}$.

and

$$
f_{ \pm}(t)=\left(1+\boldsymbol{A}_{\boldsymbol{N}_{\mathrm{CBO}}}\left(t^{\prime}\right)\right) e^{\mp T /(2 \tau)}\left(1+\boldsymbol{A}\left(1+\boldsymbol{A}_{\boldsymbol{A}_{\mathrm{CBO}}}\left(t^{\prime}\right)\right) \cos \left(\boldsymbol{\omega}_{\boldsymbol{a}} t^{\prime}+\boldsymbol{\phi}_{\boldsymbol{a}}\left(1+\boldsymbol{A}_{\boldsymbol{\phi}_{\mathrm{CBO}}}\left(t^{\prime}\right)\right)\right)\right.
$$

where $t^{\prime}$ is the shifted time

$$
\begin{gathered}
t^{\prime}=t \pm T / 2, \\
\boldsymbol{\omega}_{\boldsymbol{a}}=2 \pi f_{a}\left(1-\left(\boldsymbol{R}-R_{o f f}\right) \times 10^{-6}\right),
\end{gathered}
$$

as before, $\tau$ is the dilated muon lifetime fixed to $64.4 \mu \mathrm{s}$,

$$
\boldsymbol{A}_{\boldsymbol{N}_{\mathrm{CBO}}}(t)=\boldsymbol{A}_{\boldsymbol{N}_{\mathrm{CBO}}} e^{-t / \tau_{\mathrm{CBO}}} \cos \left(\omega_{\mathrm{CBO}} t+\boldsymbol{\phi}_{\boldsymbol{N}_{\mathrm{CBO}}}\right)
$$

is the CBO effect on $N$,

$$
\boldsymbol{A}_{\boldsymbol{A}_{\mathrm{CBO}}}(t)=\boldsymbol{A}_{\boldsymbol{A}_{\mathrm{CBO}}} e^{-t / \tau_{\mathrm{CBO}}} \cos \left(\omega_{\mathrm{CBO}} t+\phi_{\boldsymbol{A}_{\mathrm{CBO}}}\right)
$$

is the CBO effect on $A$, and

$$
\boldsymbol{A}_{\phi_{\mathrm{CBO}}}(t)=\boldsymbol{A}_{\phi_{\mathrm{CBO}}} e^{-t / \tau_{\mathrm{CBO}}} \cos \left(\omega_{\mathrm{CBO}} t+\phi_{\phi_{\mathrm{CBO}}}\right)
$$

is the $\mathrm{CBO}$ effect on $\phi_{\boldsymbol{a}}$. Once again, the $\mathrm{CBO}$ frequencies and lifetimes are fixed to the same values used in the five- and seven-parameter ratio fits.

The results of the fits are shown in Figs. 4.35-4.39, and are very compatible with the fit results obtained from the seven parameter ratio function. We find that the $g-2$ phase is modulated by $\sim 1.4 \mathrm{mrad}$, which corresponds to a modulation in detection time of $\sim 1$ 
Low-n Data Set, Nine Par. Fit
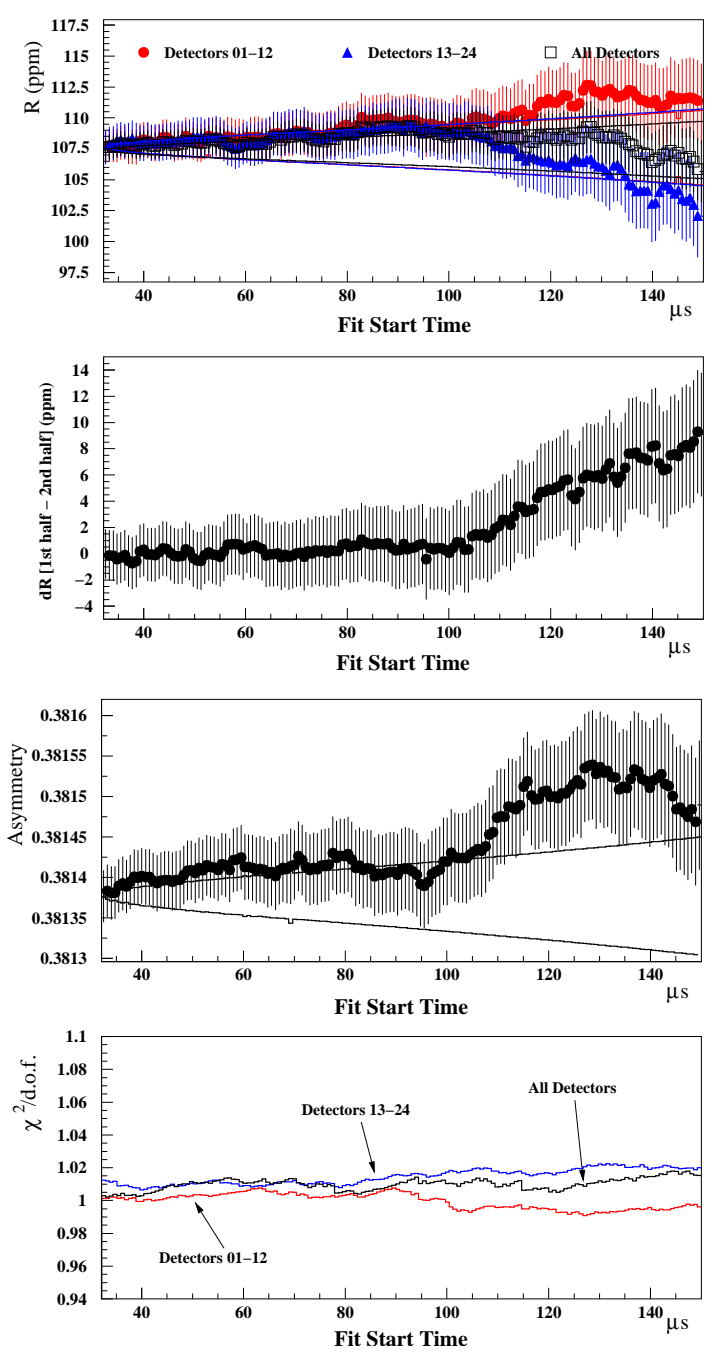

(a) Low-n data set.
High-n Data Set, Nine Par. Fit
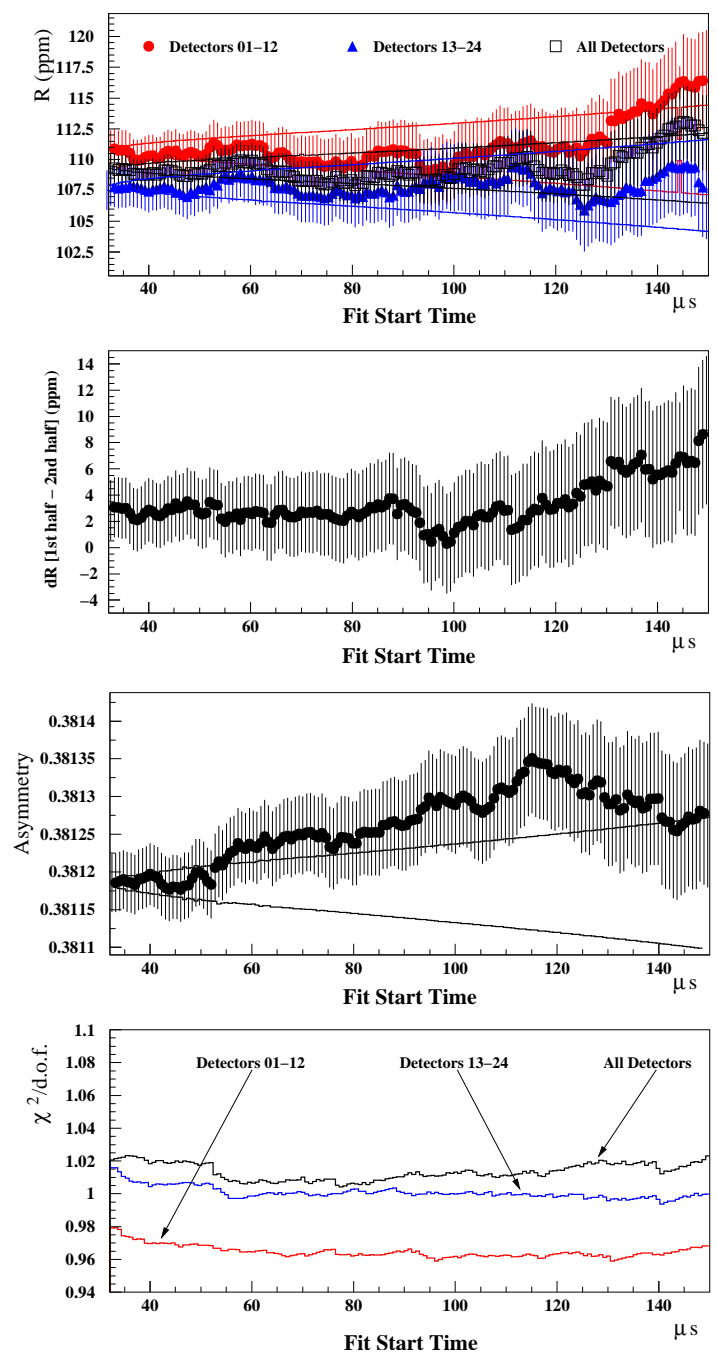

(b) High-n data set.

Figure 4.36: From top to bottom: 1. $R$ vs. fit start time for the nine parameter fit for different combinations of detectors: 1-12 (red circles), 13-24 (blue triangles), and 1-24 (black squares). 2. Difference of $\mathrm{R}$ vs. fit start time plots between first and second halves of the ring. 3. Asymmetry vs. fit start time for detector 25. 4. $\chi^{2} /$ d.o.f. vs. fit start time for different combinations of detectors. 
Low-n Data Set, Nine Par. Fit
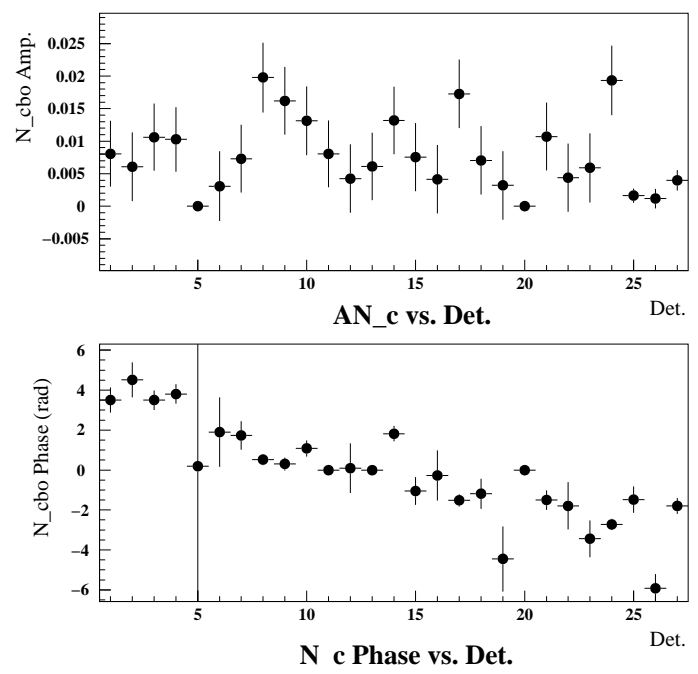

(a) Low-n data set.
High-n Data Set, Nine Par. Fit
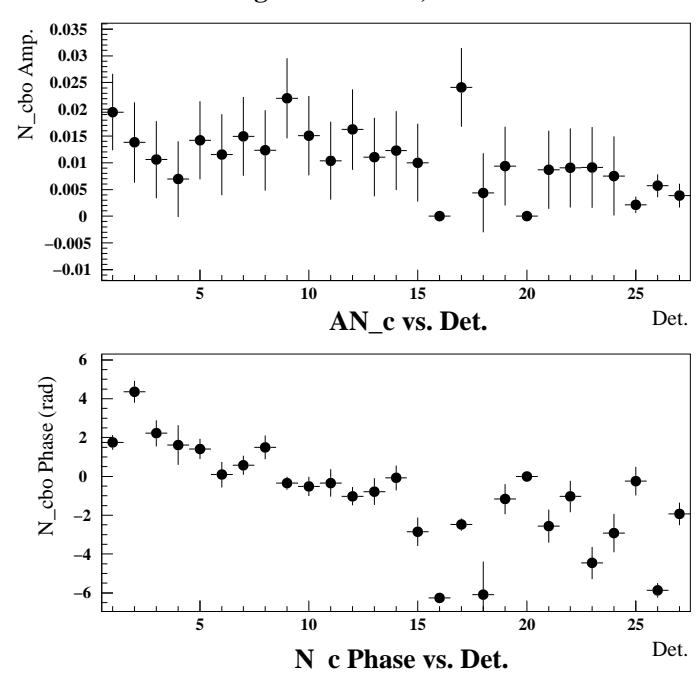

(b) High-n data set.

Figure 4.37: $N_{\mathrm{CBO}}$ term amplitudes and phases vs. Detector for the nine parameter fit. All points are taken at $\mathrm{t}=32.5 \mu \mathrm{s}$.
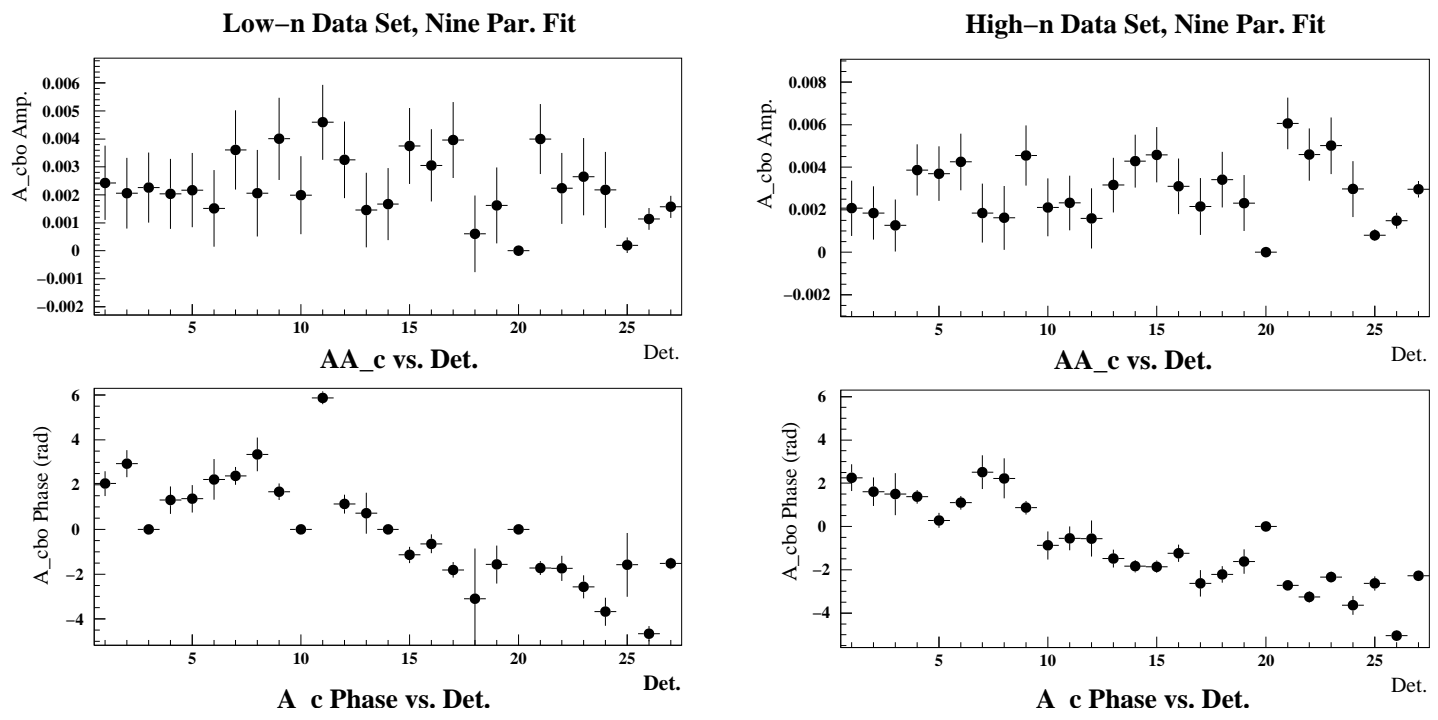

(a) Low-n data set.

(b) High-n data set.

Figure 4.38: $A_{\mathrm{CBO}}$ term amplitudes and phases vs. Detector for the nine parameter fit. All points are taken at $\mathrm{t}=32.5 \mu \mathrm{s}$. 

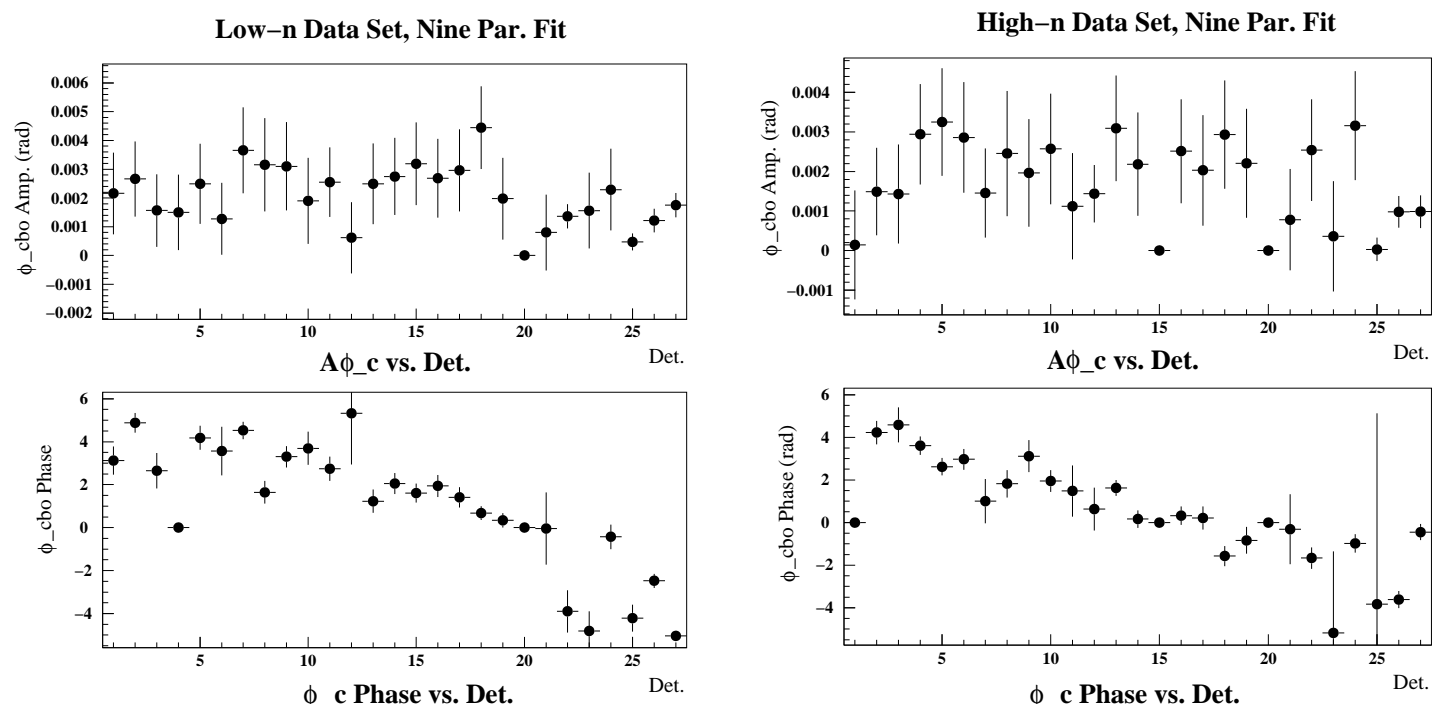

(a) Low-n data set.

(b) High-n data set.

Figure 4.39: $\phi_{\mathrm{CBO}}$ term amplitudes and phases vs. Detector for the nine parameter fit. All points are taken at $\mathrm{t}=32.5 \mu \mathrm{s}$.

ns. Simulations have shown that the expected modulation from differences in drift time alone are of the order of a few hundred picoseconds, accounting for only a fraction of the observed modulation in the $g-2$ phase. The remaining contribution to the modulation arises from acceptance effects; the average phase depends on energy, and CBO affects the average energy.

\section{Fit Results with Different Fit Start Times}

The detectors closest to the injection point see the largest neutron background and therefore the PMTs of these detectors are gated on almost $25 \mu$ s later than the PMTs of the detectors on the opposite side of the ring. If one wishes to combine the time spectra from all of the detectors into one time spectrum (eg: that of detector 25), then the earliest one may begin to fit the data is $\sim 32 \mu$ s after injection. One may reduce the statistical uncertainty if data from the detectors are fit separately using individual, earlier start times. At the end, one simply takes the weighted average value of $R$ from the individual detector fit results. This xsrequires that CBO is properly accounted for, and only a seven or nine parameter fit function is appropriate for this approach.

The individual fit start times $\left(t_{f}\right)$ were determined according to the following criteria:

- $\chi^{2} /$ d.o.f. is 'acceptable'

- fit parameters are stable

- $t_{f}>22 \mu \mathrm{s}$ (after scraping) 


\begin{tabular}{|c||c||c||c|c||c|}
\hline \multicolumn{7}{|c|}{ Fit Start Time } \\
\hline \hline Det. & 1,8 & 2 & $3,6,7$ & 4,5 & $9-24$ \\
\hline Time $(\mu \mathrm{s})$ & 27.4 & 25.3 & 29.6 & 34.0 & 23.0 \\
\hline
\end{tabular}

Table 4.2: Fit start times for the individual detectors. All fit start times are after scraping and at (or very close to) $g$-2 zero crossings.
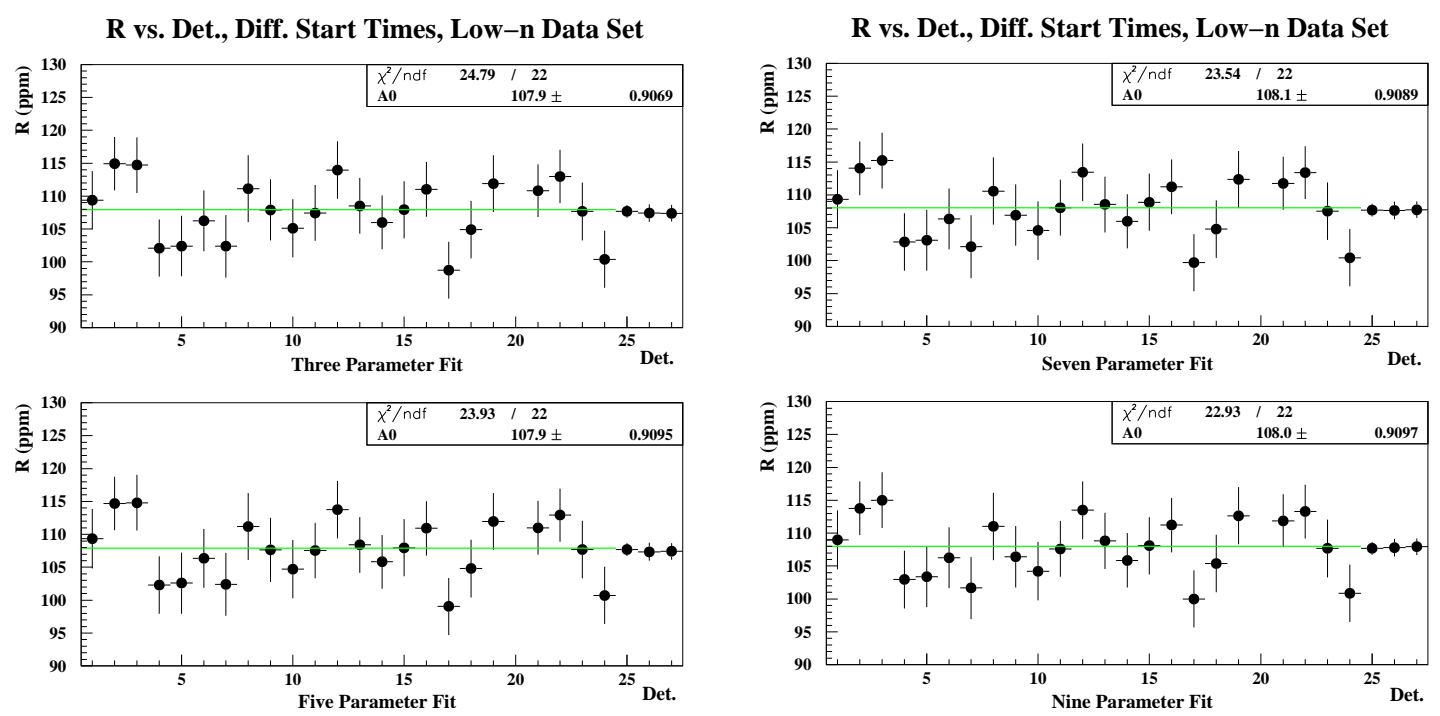

(a) Three and five parameter ratio fits.

(b) Seven and nine parameter ratio fits.

Figure 4.40: Fit result comparison of $R$ vs. Det. for the low-n data set using different fit start times for each detector.

- $t_{f}$ is a $g-2$ zero crossing

"Acceptable" $\chi^{2} /$ d.o.f. is defined as the time at which the $\chi^{2} /$ d.o.f. in the fit-time scan drops below $2.5 \sigma$ from 1 (therefore, $\left|\chi^{2}-1\right|<0.05$ ). In the case of consistently high $\chi^{2} /$ d.o.f. (greater than $2.5 \sigma$ from 1) vs. fit start time, an average $\chi^{2} /$ d.o.f. is determined from fit results between 90 and $150 \mu \mathrm{s}$, and "acceptable" $\chi^{2} /$ d.o.f. is defined as $\left|\left(\chi^{2}-\chi_{\text {late }}^{2}\right) / \chi_{\text {late }}^{2}\right|<$ 0.05 . The reason for choosing a $g$ - 2 zero crossing (where the cosine term of the time spectrum crosses zero) is because most phase-pulling effects, such as pileup and energy-scale changes, are minimized at these times. The fit start times of the detectors are listed in Table 4.2.

Figs. 4.40 and 4.41 show $\mathrm{R}$ vs. detector with different fit start times for all four fit functions. The average values of $\mathrm{R}$ for each fit is obtained by fitting a constant to the 24 detectors. Note that although the fit values of $\mathrm{R}$ vary only slightly vs. fit function, the fit $\chi^{2} /$ d.o.f. improves significantly as more and more CBO effects are fit for, going from the three parameter ratio function to the nine parameter ratio function. 
R vs. Det., Diff. Start Times, High-n Data Set
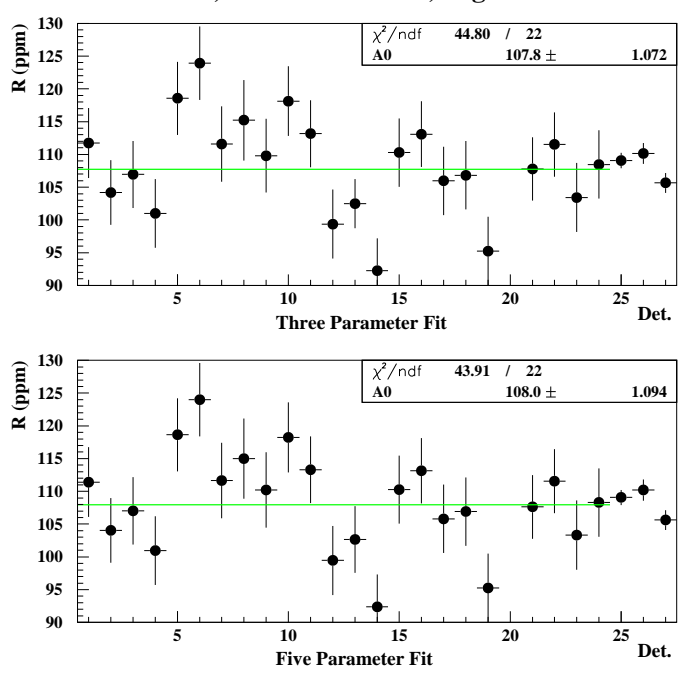

(a) Three and five parameter ratio fits.
R vs. Det., Diff. Start Times, High-n Data Set
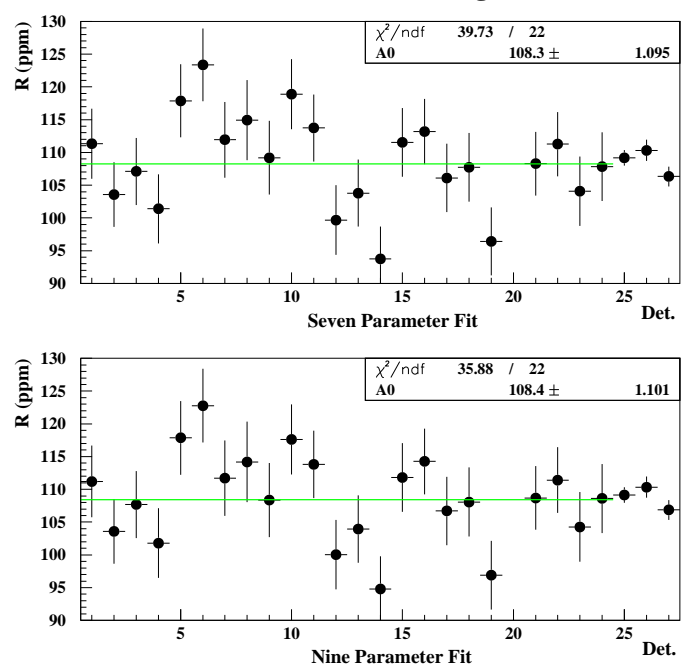

(b) Seven and nine parameter ratio fits.

Figure 4.41: Fit result comparison of $R$ vs. Det. for the high-n data set using different fit start times for each detector.

\section{Summary of Fit Results}

Table 4.3 summarizes the various fit parameters and constants used in each fit function describe above. A "free" parameter refers to one that is determined from the fit, and an empty entry means "not applicable". Since there are a great number of fit results to consider (26 detectors $\times n$-parameters for an $n$-parameter fit), the fit values for $R$ and $\chi^{2} /$ d.o.f. are summarized in Table 4.4. These tables compare $R$-values obtained from the average over all 23 detector $R$-values at $32.5 \mu \mathrm{s}$, detector 25 at $32.5 \mu \mathrm{s}$, and the average over all 23 detector $R$-values with the different start times given in Table 4.2.

The two $R$-values obtained from fits at $32.5 \mu$ s (the average over all detectors and from the fit to the sum of all detectors) agree very well, within $0.2 \mathrm{ppm}$. However, there is a larger difference between the average $R$ obtained from fits to data from detectors at the same fit time $(32.5 \mu \mathrm{s})$ and fits using different individual start times. For the low-n data set, the difference is $\sim 0.4 \mathrm{ppm}$, and for the high-n data set, the difference is $\sim 0.8 \mathrm{ppm}$. The average start time of the fits to data with different start times is $\simeq 25.5 \mu \mathrm{s}$, whereas the fits to data with the same start time began at $32.5 \mu \mathrm{s}$. Taking into account the extra $7 \mu$ s of data, the allowed deviation in $R$ is $\sim 30 \%$ of the statistical uncertainty of the later fit. Therefore the differences of $0.4 \mathrm{ppm}$ and $0.8 \mathrm{ppm}$ represent a deviation in $R$ by $\sim 1.3$ $\sigma$ and $\sim 2.5 \sigma$. While the latter difference is worrisome, as will be shown, no systematic effects were found that could account for the difference. Therefore, we conclude that the difference is statistical in nature.

An aggressive approach of obtaining the final $R$-value from fits to data from individual detectors at different start times is chosen in this analysis. This reduces the statistical 


\begin{tabular}{|c||c||c|c||c|}
\hline \multicolumn{5}{|c|}{ Fit Parameter Status } \\
\hline \hline Fit & 3 Par. & 5 Par. & 7 Par. & 9 Par. \\
\hline \hline$A$ & Free & Free & Free & Free \\
\hline$R$ & Free & Free & Free & Free \\
\hline$\phi$ & Free & Free & Free & Free \\
\hline $\begin{array}{c}\text { constant/ } \\
\tau_{\mu}\end{array}$ & $2.874 \times 10^{-4}$ & 64.4 & 64.4 & 64.4 \\
\hline$A_{N_{\mathrm{CBO}}}$ & - & Free & Free & Free \\
\hline$\phi_{N_{\mathrm{CBO}}}$ & - & Free & Free & Free \\
\hline$A_{A_{\mathrm{CBO}}}$ & - & - & Free & Free \\
\hline$\phi_{A_{\mathrm{CBO}}}$ & - & - & Free & Free \\
\hline$A_{\phi_{\mathrm{CBO}}}$ & - & - & - & Free \\
\hline$\phi_{\phi_{\mathrm{CBO}}}$ & - & - & - & Free \\
\hline $\begin{array}{c}f_{\mathrm{CBO}}(\mathrm{kHz}) \\
\text { low-n }\end{array}$ & - & 419.1 & 419.1 & 419.1 \\
\hline $\begin{array}{c}\tau_{\mathrm{CBO}}(\mu \mathrm{s}) \\
\text { low-n }\end{array}$ & - & 92.1 & 92.1 & 92.1 \\
\hline $\begin{array}{c}f_{\mathrm{CBO}}(\mathrm{kHz}) \\
\text { high-n }\end{array}$ & - & 490.6 & 490.6 & 490.6 \\
\hline $\begin{array}{c}\tau_{\mathrm{CBO}}(\mu \mathrm{s}) \\
\text { high-n }\end{array}$ & - & 130.1 & 130.1 & 130.1 \\
\hline \multicolumn{2}{|c||}{} & & & \\
\hline
\end{tabular}

Table 4.3: Summary of fit parameter status in each ratio fit. "Free" means that the parameter is determined from the fit, number/values in an entry means that the fit parameter was held fixed to that value in the fit, and an empty entry means not applicable.

uncertainty on $\omega_{a}$ by at least $6 \%$. The question remains as to which function to use in the final analysis of the data. Clearly either the seven- or the nine-parameter ratio function must be used in order to account effectively for CBO effects. The decision to use the nine-parameter ratio function is based on three key points:

- The fit to a constant of $R$ vs. detector obtained from the nine parameter ratio fits has a better $\chi^{2} /$ d.o.f. than that obtained from all other ratio fits (see Figs. 4.40 and 4.41).

- The nine parameter ratio function accounts for all known CBO effects.

- There are no strong correlations between $R$ and the other 8 fit parameters in the nine-parameter ratio function.

The second point is clear: there is a physical model which predicts the existence of the $N_{\text {cbo }}, A_{\text {cbo }}$ and $\phi_{\text {cbo }}$ terms. Furthermore, the existence of these terms have been verified by both simulation and by fits to data. It is clear that the seven and nine parameter ratio fits effectively eliminate the half-ring effect, as demonstrated in Fig. 4.42. Oscillations at the frequency $f_{\mathrm{CBO}}-2 f_{a}$, or $\sim 30 \mathrm{kHz}$, are clearly seen in the three and five parameter fits whereas they are not as apparent in the seven and nine parameter. Another comparison of 

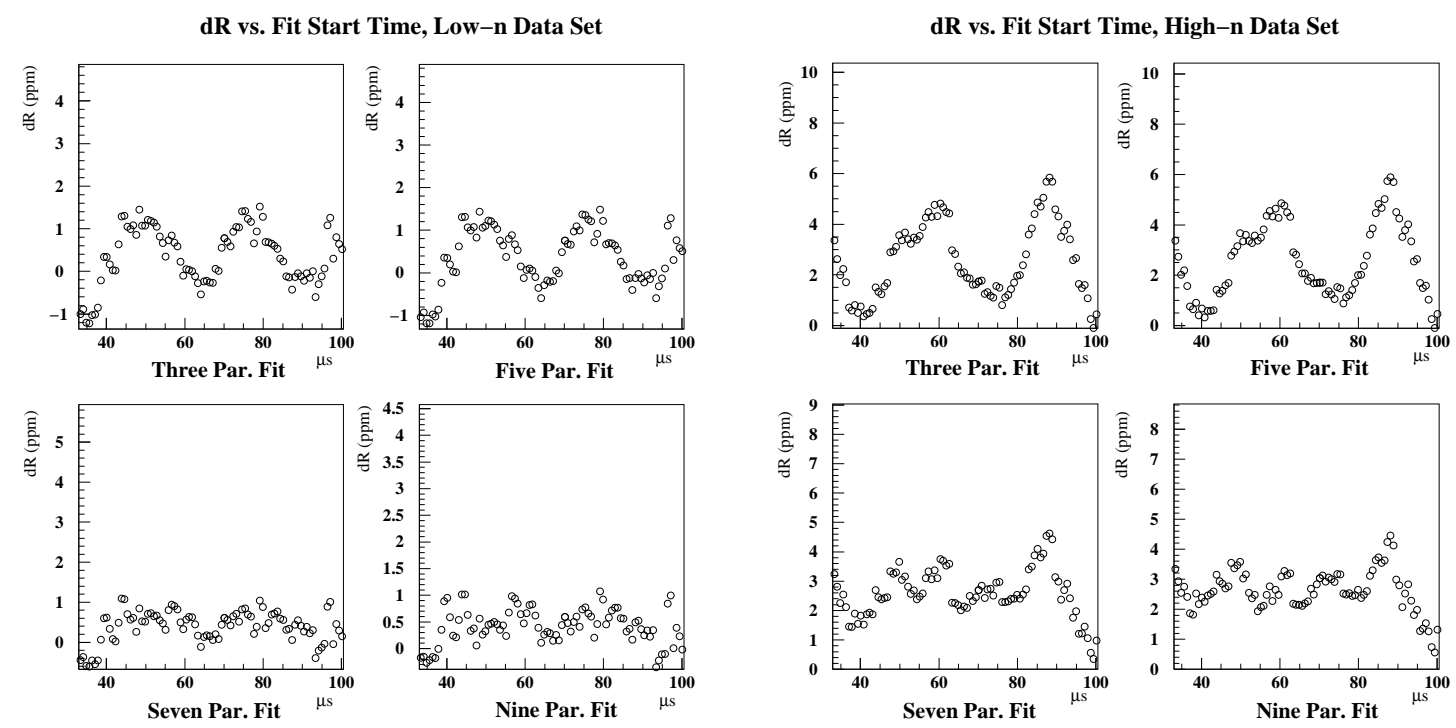

(a) Low-n data set.
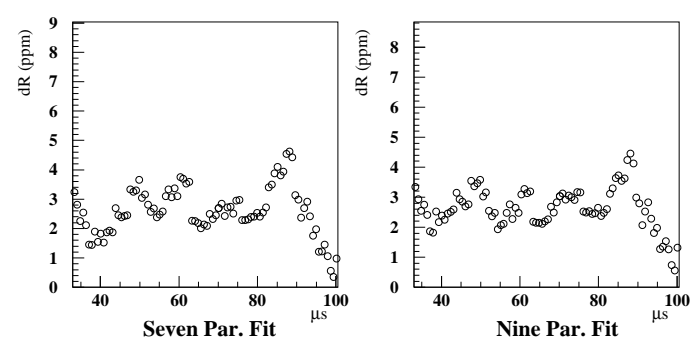

(b) High-n data set.

Figure 4.42: Difference between first-half and second-half of the ring of $R$ vs. fit time for various fit functions.
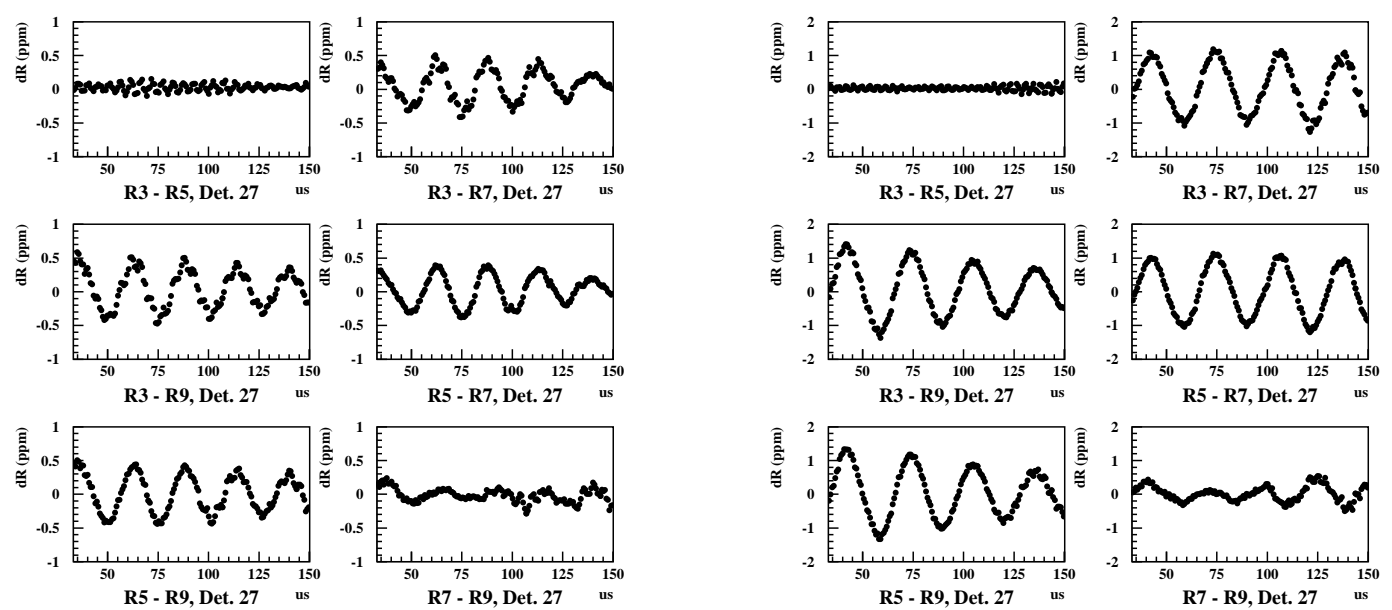

(a) Low-n data set.
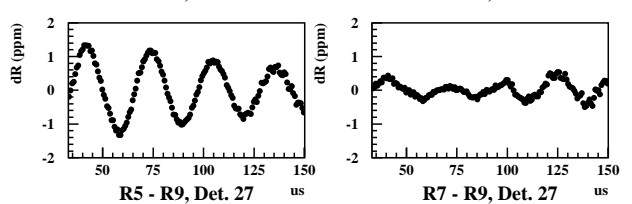

(b) High-n data set.

Figure 4.43: Difference between various fit functions of $R$ vs. fit time plots of detector 27 . $\mathrm{R} n$ corresponds to the $n$-parameter ratio function. 


\begin{tabular}{|c|c|c|c|c|}
\hline \multicolumn{5}{|c|}{ Comparison of Fit R Values - Low-n Data Set } \\
\hline Fit & 3 Par. & 5 Par. & 7 Par. & 9 Par. \\
\hline $\begin{array}{c}\quad\langle R\rangle\left(t_{f}=32.5 \mu \mathrm{s}\right) \\
\text { (constant fit to } R \text { vs. Det.) }\end{array}$ & $\begin{array}{c}107.6 \\
\pm 0.94\end{array}$ & $\begin{array}{l}107.5 \\
\pm .97\end{array}$ & $\begin{array}{l}107.5 \\
\pm 0.97\end{array}$ & $\begin{array}{l}107.6 \\
\pm 0.96\end{array}$ \\
\hline $\begin{array}{c}\chi^{2} / \text { d.o.f. of } \\
\text { constant fit to } R \text { vs. Det. }\end{array}$ & 0.833 & 0.828 & 0.775 & 0.796 \\
\hline $\begin{array}{c}\text { Mean } \chi^{2} / \text { d.o.f. } \\
\text { over } 23 \text { detectors }\end{array}$ & 1.011 & 1.010 & 1.010 & 1.010 \\
\hline $\operatorname{RMS}\left(\chi^{2} /\right.$ d.o.f. $)$ & 0.039 & 0.038 & 0.039 & 0.039 \\
\hline $\begin{array}{l}\mathrm{R} @ 32.5 \mu \mathrm{s} \\
\text { for Det. } 25\end{array}$ & $\begin{array}{l}107.4 \\
\pm 0.96\end{array}$ & $\begin{array}{c}107.6 \\
\pm 0.95\end{array}$ & $\begin{array}{l}107.3 \\
\pm 0.97\end{array}$ & $\begin{array}{l}107.4 \\
\pm 0.97\end{array}$ \\
\hline$\chi^{2} /$ d.o.f. & 1.006 & 0.995 & 1.003 & 1.003 \\
\hline $\begin{array}{c}\langle R\rangle\left(\text { diff. } t_{f} \text { 's) }\right. \\
\text { (constant fit to } R \text { vs. Det.) }\end{array}$ & $\begin{array}{l}107.9 \\
\pm 0.91\end{array}$ & $\begin{array}{l}107.9 \\
\pm 0.91\end{array}$ & $\begin{array}{l}108.1 \\
\pm 0.91\end{array}$ & $\begin{array}{l}108.0 \\
\pm 0.91\end{array}$ \\
\hline $\begin{array}{c}\chi^{2} / \text { d.o.f. of } \\
\text { constant fit to } R \text { vs. Det. }\end{array}$ & 1.113 & 1.088 & 1.070 & 1.042 \\
\hline $\begin{array}{c}\text { Mean } \chi^{2} / \text { d.o.f. } \\
\text { over } 23 \text { detectors }\end{array}$ & 1.016 & 1.015 & 1.014 & 1.014 \\
\hline $\operatorname{RMS}\left(\chi^{2} /\right.$ d.o.f. $)$ & 0.039 & 0.039 & 0.039 & 0.039 \\
\hline \multicolumn{5}{|c|}{ Comparison of Fit R Values - High-n Data Set } \\
\hline Fit & 3 Par. & 5 Par. & 7 Par. & 9 Par. \\
\hline $\begin{array}{c}\langle R\rangle\left(t_{f}=32.5 \mu \mathrm{s}\right) \\
\text { (constant fit to } R \text { vs. Det.) }\end{array}$ & $\begin{array}{l}109.2 \\
\pm 1.17\end{array}$ & $\begin{array}{c}109.0 \\
\pm 1.16\end{array}$ & $\begin{array}{c}109.2 \\
\pm 1.16\end{array}$ & $\begin{array}{l}109.2 \\
\pm 1.17\end{array}$ \\
\hline $\begin{array}{c}\chi^{2} / \text { d.o.f. of } \\
\text { constant fit to } R \text { vs. Det. }\end{array}$ & 1.584 & 1.597 & 1.525 & 1.492 \\
\hline $\begin{array}{c}\text { Mean } \chi^{2} / \text { d.o.f. } \\
\text { over } 23 \text { detectors }\end{array}$ & 1.013 & 1.012 & 1.011 & 1.010 \\
\hline $\operatorname{RMS}\left(\chi^{2} /\right.$ d.o.f. $)$ & 0.025 & 0.024 & 0.024 & 0.024 \\
\hline $\begin{array}{l}\mathrm{R} @ 32.5 \mu \mathrm{s} \\
\text { for Det. } 25\end{array}$ & $\begin{array}{c}109.2 \\
\pm 1.16\end{array}$ & $\begin{array}{c}109.2 \\
\pm 1.13\end{array}$ & $\begin{array}{c}109.3 \\
\pm 1.16\end{array}$ & $\begin{array}{r}109.3 \\
\pm 1.17\end{array}$ \\
\hline$\chi^{2} /$ d.o.f. & 1.023 & 1.020 & 1.020 & 1.021 \\
\hline $\begin{array}{c}\langle R\rangle\left(\text { diff. } t_{f} \text { 's) }\right. \\
\text { (constant fit to } R \text { vs. Det. }\end{array}$ & $\begin{array}{l}107.8 \\
\pm 1.07\end{array}$ & $\begin{array}{c}108.0 \\
\pm 1.09\end{array}$ & $\begin{array}{c}108.3 \\
\pm 1.10\end{array}$ & $\begin{array}{r}108.4 \\
\pm 1.10\end{array}$ \\
\hline $\begin{array}{c}\chi^{2} / \text { d.o.f. of } \\
\text { constant fit to } R \text { vs. Det. }\end{array}$ & 2.036 & 1.996 & 1.806 & 1.631 \\
\hline $\begin{array}{l}\text { Mean } \chi^{2} / \text { d.o.f. } \\
\text { over } 23 \text { detectors }\end{array}$ & 1.029 & 1.028 & 1.026 & 1.026 \\
\hline $\operatorname{RMS}\left(\chi^{2} /\right.$ d.o.f. $)$ & 0.026 & 0.025 & 0.025 & 0.025 \\
\hline
\end{tabular}

Table 4.4: Brief summary of the current results from the various functions fit to the two data sets. The $\chi^{2} /$ d.o.f. is from the result of fitting $R$ vs. Det. to a constant, whereas the mean and $\operatorname{RMS}\left(\chi^{2} /\right.$ d.o.f. $)$ are taken from the distribution of fit $\chi^{2}$ 's from each detector. 


\begin{tabular}{|c|c|c|c|c|c|c|c|c|c|}
\hline \multicolumn{10}{|c|}{ Correlation Matrix for Nine Parameter Fit, Low-n } \\
\hline Par. & $\bar{A}$ & $R$ & $\phi_{a}$ & $A_{N_{\mathrm{CBO}}}$ & $\phi_{N_{\mathrm{CBO}}}$ & $A_{A_{\mathrm{CBO}}}$ & $\phi_{A_{\mathrm{CBO}}}$ & $A_{\phi_{\mathrm{CBO}}}$ & $\phi_{\phi_{\mathrm{CBO}}}$ \\
\hline $\bar{A}$ & 1.000 & -0.005 & -0.007 & 0.000 & 0.013 & 0.049 & -0.054 & -0.046 & 0.045 \\
\hline$R$ & -0.005 & 1.000 & 0.867 & -0.024 & 0.001 & 0.051 & 0.041 & -0.032 & -0.033 \\
\hline$\phi_{a}$ & -0.007 & 0.867 & 1.000 & -0.033 & 0.001 & 0.068 & 0.057 & -0.042 & -0.046 \\
\hline$A_{N_{\mathrm{CBO}}}$ & 0.000 & -0.024 & -0.033 & 1.000 & -0.005 & 0.002 & -0.013 & -0.006 & 0.006 \\
\hline$\phi_{N_{\mathrm{CBO}}}$ & 0.013 & 0.001 & 0.001 & -0.005 & 1.000 & 0.009 & 0.000 & 0.010 & 0.002 \\
\hline$A_{A_{\mathrm{CBO}}}$ & 0.049 & 0.051 & 0.068 & 0.002 & 0.009 & 1.000 & 0.001 & -0.011 & -0.012 \\
\hline$\phi_{A_{\mathrm{CBO}}}$ & -0.054 & 0.041 & 0.057 & -0.013 & 0.000 & 0.001 & 1.000 & 0.009 & 0.001 \\
\hline$A_{\phi_{\mathrm{CBO}}}$ & -0.046 & -0.032 & -0.042 & -0.006 & 0.010 & -0.011 & 0.009 & 1.000 & -0.004 \\
\hline$\phi_{\phi_{\mathrm{CBO}}}$ & 0.045 & -0.033 & -0.046 & 0.006 & 0.002 & -0.012 & 0.001 & -0.004 & 1.000 \\
\hline \multicolumn{10}{|c|}{ Correlation Matrix for Nine Parameter Fit, High-n } \\
\hline Par. & $A$ & $R$ & $\phi_{a}$ & $A_{N_{\mathrm{CBO}}}$ & $\phi_{N_{\mathrm{CBO}}}$ & $A_{A_{\mathrm{CBO}}}$ & $\phi_{A_{\mathrm{CBO}}}$ & $A_{\phi_{\mathrm{CBO}}}$ & $\phi_{\phi_{\mathrm{CBO}}}$ \\
\hline$\overline{\bar{A}}$ & 1.000 & 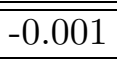 & $\overline{0.000}$ & 0.004 & -0.015 & 0.021 & 0.081 & 0.071 & 0.025 \\
\hline$R$ & -0.001 & 1.000 & 0.861 & 0.007 & 0.014 & -0.085 & 0.003 & -0.041 & 0.067 \\
\hline$\phi_{a}$ & 0.000 & 0.861 & 1.000 & 0.010 & 0.019 & -0.114 & 0.007 & -0.041 & 0.092 \\
\hline$A_{N_{\mathrm{CBO}}}$ & 0.004 & 0.007 & 0.010 & 1.000 & 0.004 & -0.013 & 0.027 & -0.018 & -0.021 \\
\hline$\phi_{N_{\mathrm{CBO}}}$ & -0.015 & 0.014 & 0.019 & 0.004 & 1.000 & -0.035 & 0.001 & 0.020 & 0.026 \\
\hline$A_{A_{\mathrm{CBO}}}$ & 0.021 & -0.085 & -0.114 & -0.013 & -0.035 & 1.000 & -0.005 & -0.011 & -0.016 \\
\hline$\phi_{A_{\mathrm{CBO}}}$ & 0.081 & 0.003 & 0.007 & 0.027 & 0.001 & -0.005 & 1.000 & -0.005 & -0.007 \\
\hline$A_{\phi_{\mathrm{CBO}}}$ & 0.071 & -0.041 & -0.041 & -0.018 & 0.020 & -0.011 & -0.005 & 1.000 & 0.070 \\
\hline$\phi_{\phi_{\mathrm{CBO}}}$ & 0.025 & 0.067 & 0.092 & -0.021 & 0.026 & -0.016 & -0.007 & 0.070 & 1.000 \\
\hline
\end{tabular}

Table 4.5: Correlation Matrix of the nine fit parameters of the full physics function for the low-n data set. Values are from the fit to detector 25 , around $40 \mu \mathrm{s}$. 
the fit functions is the difference of $R$ vs. fit time between the various fit functions, shown in Fig. 4.43 for detector 27. For the sake of clarity, the error bars are not plotted. Since there are 4 different fit functions used, the differences shown are between the three and five parameter fits (R3-R5), the three and seven parameter fits (R3-R7), the three and nine parameter fits (R3-R9), the five and seven parameter fits (R5-R7), and so on. The differences between $R$-values found from the three and five parameter ratio functions and those found using the seven and nine parameter functions is stunningly clear. Although not shown here, the effect is still evident when all data are summed (detector 25); the amplitude of the oscillations is $\sim 0.3 \mathrm{ppm}$ for the low n-value data and $\sim 0.8 \mathrm{ppm}$ for the high $\mathrm{n}$-value data.

The last point is verified by Table 4.5, which shows the correlation coefficient matrix of all free parameters used in the nine parameter ratio function, as determined by MINUIT. We see that besides the $g-2$ phase, there are no other parameters that are strongly correlated to the $g$-2 frequency. This is an important and distinguishing feature of the fit function's ability to describe the data.

The final $R$-value is therefore obtained from an average over fits to five different time spectra, each using a different random seed to eliminate the fast rotation. Fig. 4.44 shows one of the five $R$ vs. Detector plots used to determine $\langle R\rangle$ for each data set (Golden low$\mathrm{n}$, Golden high-n and Silver+Bronze low-n). Averaging over five random seeds, our final $R$-values are: $R_{\text {low }-\mathrm{n}}=108.21 \pm 0.91 \mathrm{ppm}, R_{\mathrm{high}-\mathrm{n}}=108.42 \pm 1.10 \mathrm{ppm}$ and $R_{\mathrm{S}+\mathrm{B}}=$ $110.55 \pm 3.73$.

\subsection{Systematic Errors on $\omega_{a}$}

\subsubsection{Energy Bin Analysis}

By dividing the data up into energy bins we can see the energy-dependence of certain fit parameters, specifically the asymmetry and CBO parameters. Ideally the precession frequency, or $R$, is independent of the observed decay electron's energy.

The data were divided up into seven energy bins, $200 \mathrm{MeV}$ wide. However, since the lower energy cut is typically around $1.9 \mathrm{GeV}$ and the upper energy cut is fixed at $3.2 \mathrm{GeV}$, many individual detector fits to data in the seventh bin failed for lack of data. Therefore, in the following plots we will show results for the first six energy bins only.

Fig. 4.45 shows the fit results for the two data sets. The data from the individual detectors were fit using the nine-parameter fit function at each detector's corresponding fit start time. $\langle R\rangle(E)$ is then determined by fitting $R$ versus detector to a constant. The $\langle R\rangle$ vs. energy plots are fit to a constant. The values of $\langle R\rangle$ are very consistent across energy bins for both $n$-values, and the average over all energy-bins agrees very well with the results obtained using only one energy bin. The lower left plots show the fit asymmetry vs. energy bin. The values for the asymmetry are taken from fits to detector 27 , the second half of the ring, with a fit start time of $\sim 25 \mu$ s after injection. The three plots on the right of Figs. 4.45 and 4.45 show the amplitudes of the three CBO effects: $N_{\mathrm{CBO}}, A_{\mathrm{CBO}}$ and $\phi_{\mathrm{CBO}}$. Although the amplitudes of the effects are in relative agreement with each other between data sets, 


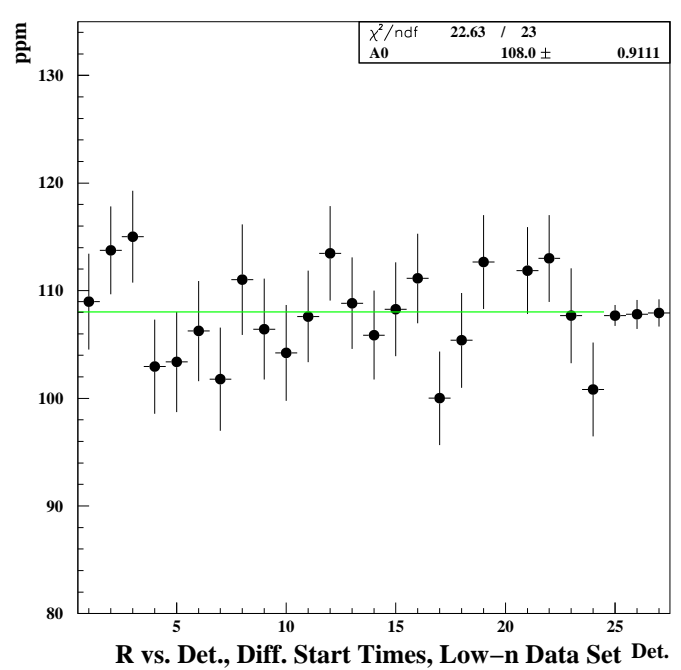

(a) Low-n data set.

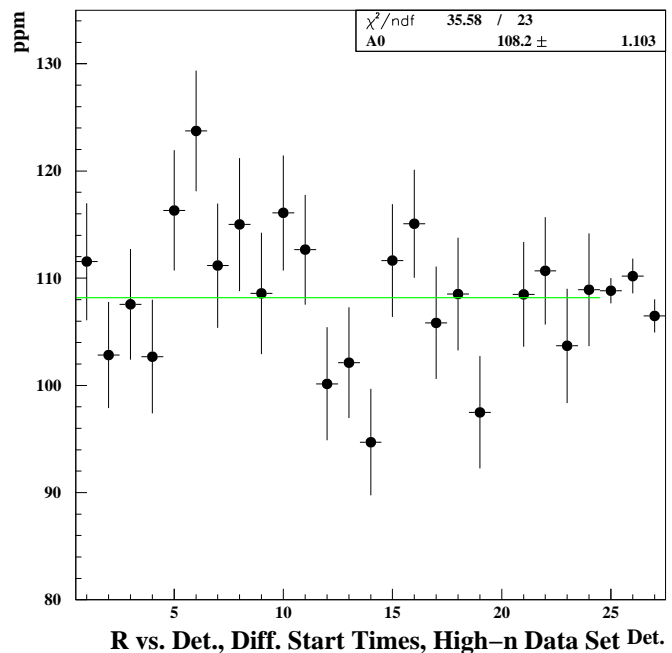

(b) High-n data set.

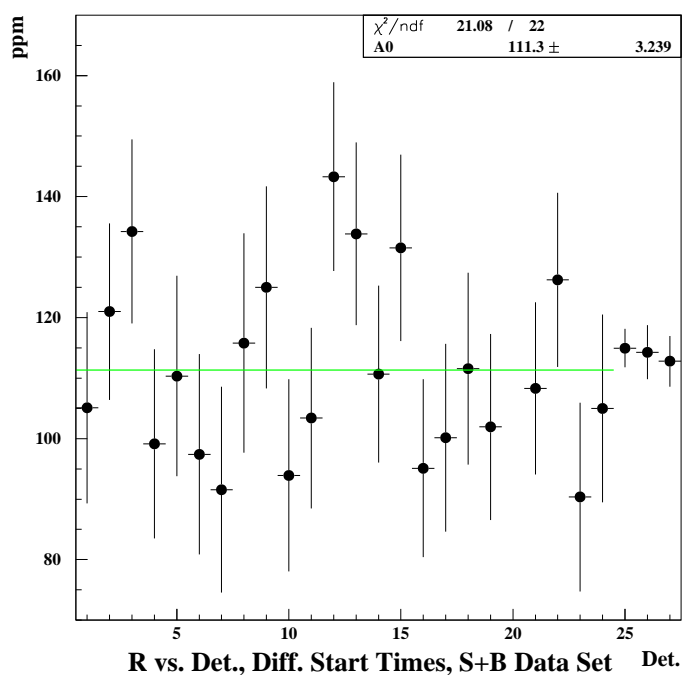

(c) $\mathrm{S}+\mathrm{B}$ data set.

Figure 4.44: $R$ vs. Detector for the low-n (Golden and Silver+Bronze) and high-n data sets, as determined using different start times for various detectors, using the nine-parameter ratio function. $R$ vs. Detector is fit to a constant to determine the average value $R$. Results for only one random seed are shown. 


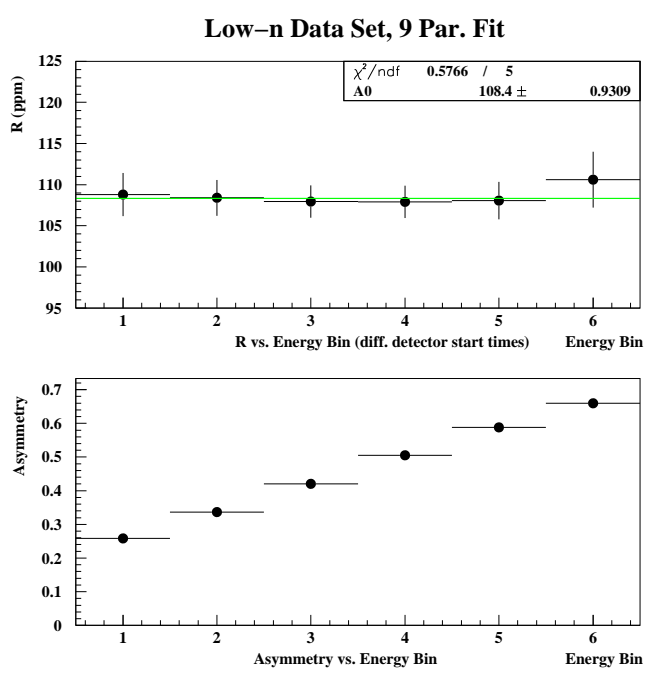

(a) $\langle R\rangle$ vs. energy bin (top) and fit asymmetry vs. energy bin.
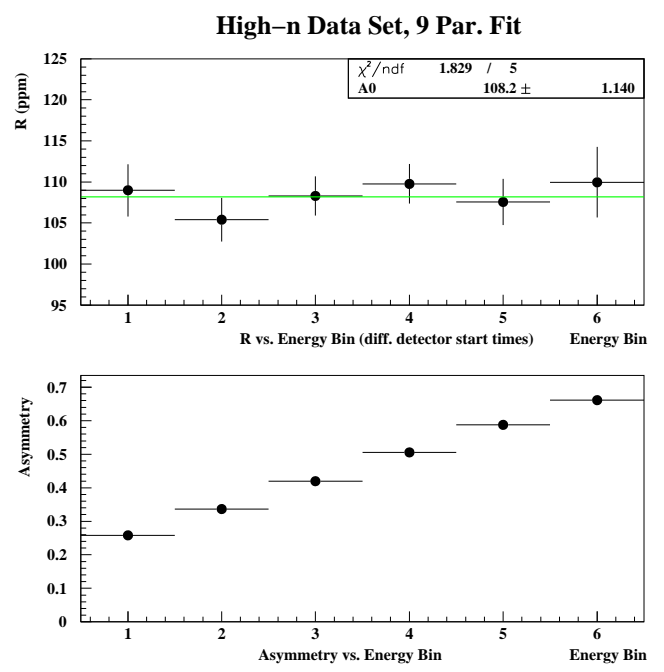

(c) $\langle R\rangle$ vs. energy bin (top) and fit asymmetry vs. energy bin.
Low-n Data Set, 9 Par. Fit
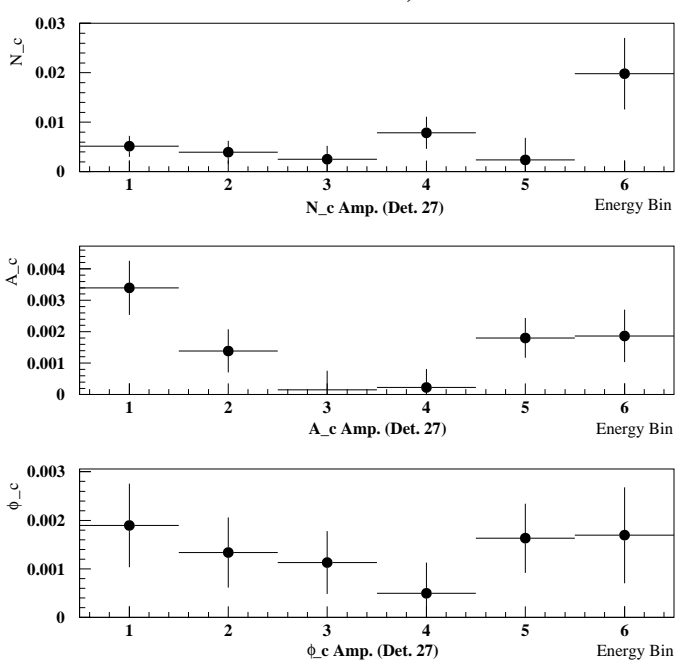

(b) CBO amplitudes vs. energy bin.

High-n Data Set, 9 Par. Fit
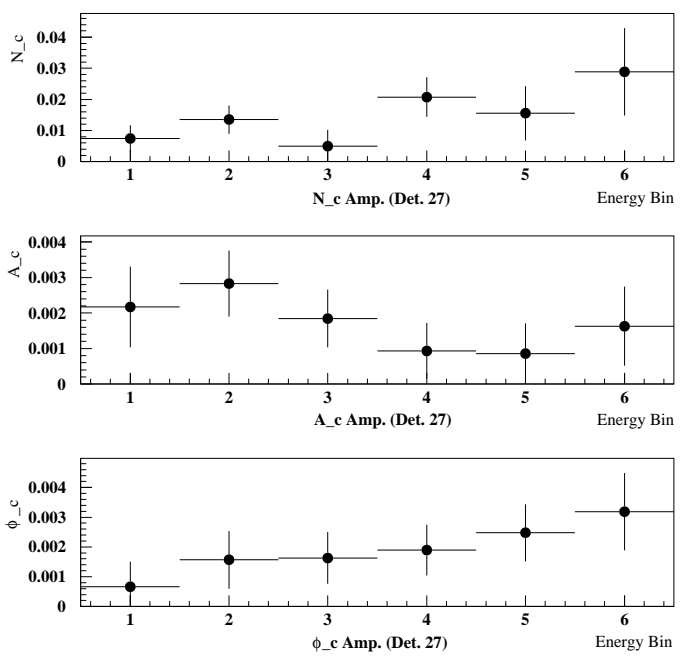

(d) CBO amplitudes vs. energy bin.

Figure 4.45: Various parameters as functions of energy bin obtained from fits to the low-n and high-n data sets. Each value of $\langle R\rangle$ is obtained from a fit to $R$ vs. detector using different start times for each detector. Energy bin 1 corresponds roughly to $1.9 \mathrm{GeV}$, and each bin is $200 \mathrm{MeV}$ wide. 

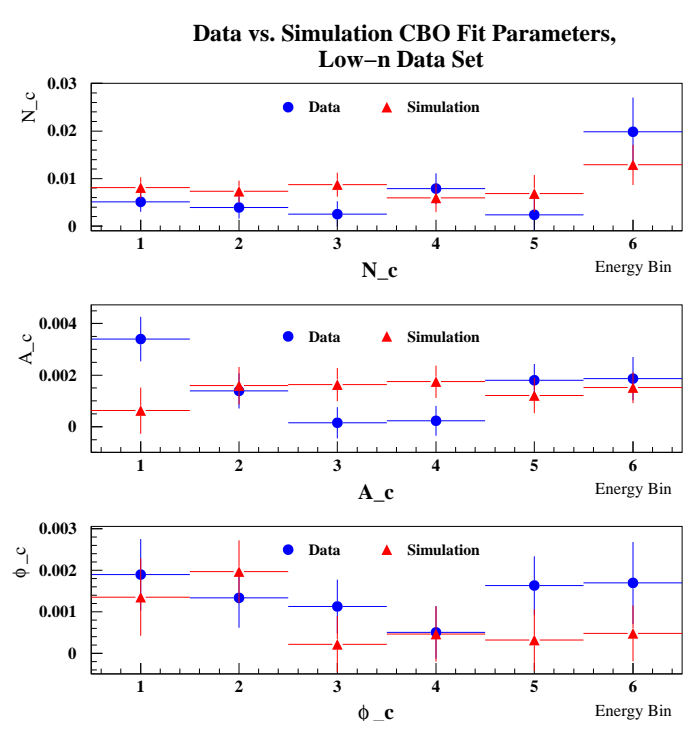

(a) Low n-value data set.
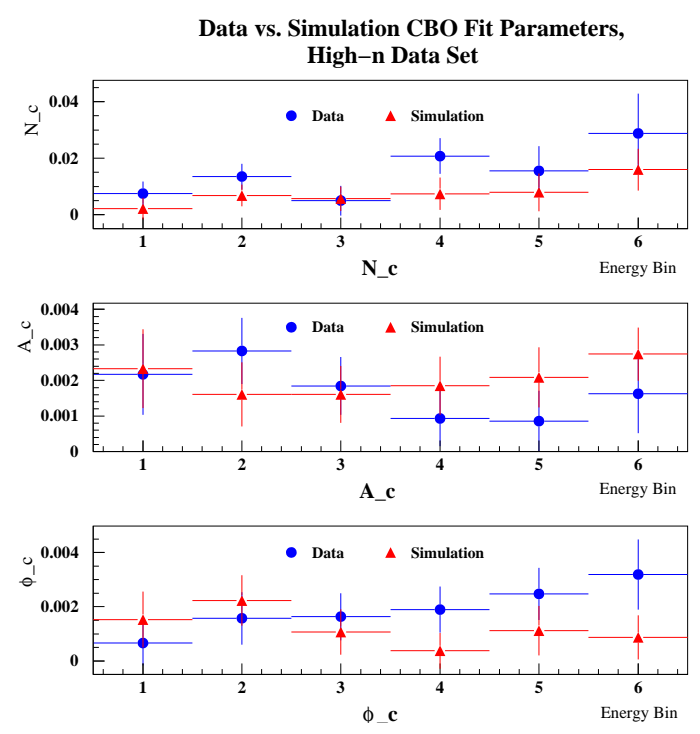

(b) High n-value data set.

Figure 4.46: Comparison between G2GEANT simulated data (red triangles) and actual data (blue circles).

the trends of the amplitudes as a function of energy bin differ quite a bit, especially for the $\phi_{\mathrm{CBO}}$ term.

To check our energy-binned results, two G2GEANT-simulated data sets were produced by R. Carey, each with the corresponding n-values and number of decay electrons of the true data sets. The simulation completely describes the geometrical and physical characteristics of the ring and detectors, decay electrons are tracked from their point of decay to a detector. The simulation does not, however, fully simulate the electromagnetic showers in the calorimeter; instead, it uses a faster parameterization (look-up table) of the detector response to the energy of the decay electron. Using this parameterization, the observed energy agrees to within $1 \%$ of the fully simulated observed energy in the center of the detector and within $10 \%$ near the edges.

Energy-binned ratio time spectra of the simulated data were produced and fit to the nine parameter ratio function. The bin-width of the energy bins of the simulated data is $200 \mathrm{MeV}$, and the minimum energy is $2 \mathrm{GeV}$. However, since the simulated energy spectrum does not perfectly match the energy spectrum obtained from true data, one cannot expect perfect agreement between the simulated and data energy-bin studies. Nevertheless, as Fig. 4.46 shows, there is in general good agreement between the data and simulation. All fit results agree within $\sim 2 \sigma$.

However, as was first demonstrated by C. Polly, there is a rather poor degree of consistency of $R$ vs. energy in the high-n data set, when $R$ is obtained from fits to detector 25 . This is equivalent to fitting the individual detector data at the same start time. $R$ is not flat vs. energy, and unfortunately the fit results are at a level for which it is very difficult to 


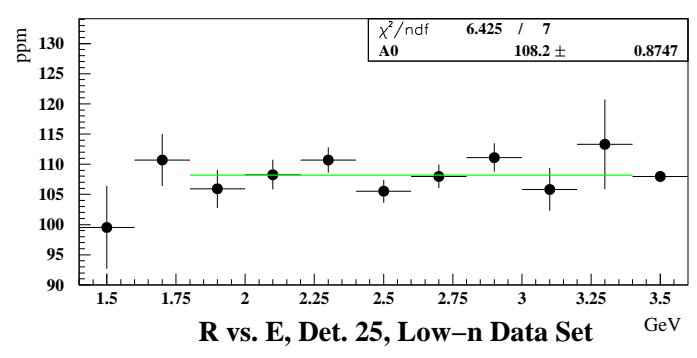

(a) Low n-value data set.

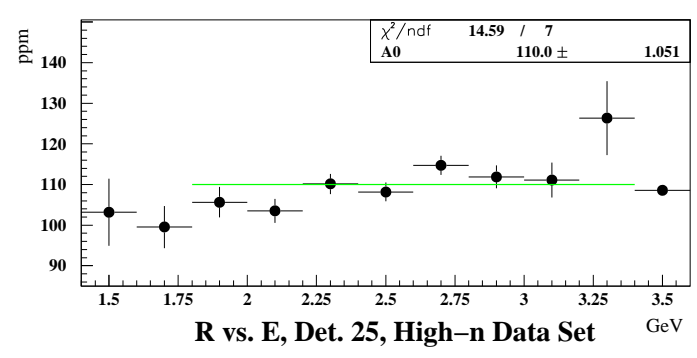

(b) High n-value data set.

Figure 4.47: $R$ vs. Energy bin for the low-n (left) and high-n (right) data sets. The lines represent fits to a constant in the energy range 1.8-3.4 GeV. Results are from fits to the three parameter ratio at $\sim 33 \mu \mathrm{s}$, however the results change very little when the nine parameter fit function is used.

determine if there is a systematic problem or simply statistical fluctuations. Polly's findings were confirmed by every $\omega_{a}$ analyzer, with varying degrees of consistency. Furthermore, one finds similar results for $\langle R\rangle$ vs. $E$, where $\langle R\rangle$ is the average over 23 detectors.

Fig. 4.47 shows $R$ vs. $E$ for the low-n and high-n data sets Both $R$ vs. $E$ plots are fit to a constant in the energy range $1.8-3.4 \mathrm{GeV}$. We see that the $\chi^{2}$ of the fit of a constant for the low-n data set has a perfectly acceptable value, whereas the same fit to the high-n data set has a $\chi^{2}$ of $\sim 2.1$. It should be noted that similar results are obtained independent of the fit function used; a comparison of $R$ vs. $E$ as determined from a nine parameter ratio fit and from a three parameter ratio fit found little differences between the two.

To further investigate the behavior of $R$ vs. $E$ in both the low- and high-n data sets, the data were split up into subsets according to chronological order. During the 2001 run, data were taken with alternating value of $n$, so the first subset is of low-n, the second subset is of high-n, the third subset is of low-n, etc. The subsets are henceforth labeled L1, H1, L2, H2, etc., where "L" corresponds to low-n, "H" corresponds to high-n, and the number following the letter corresponds to the subset of that data set. $R$ vs. $E$ was then determined for each subset, and two subsets, H2 and L3, showed strange behavior of $R$ vs. $E$, as evidenced by the fit $\chi^{2}$ 's of $R$ vs. $E$ to a constant. However, after performing extensive systematic studies and eliminating many models, no "smoking gun" was found that could explain the $R$ vs. $E$ behavior in the high-n (and in particular H2) data set (see [94] for further details). We therefore claim that the behavior is of a statistical nature.

\subsubsection{Energy Scale Changes}

To study possible systematic effects of the applied energy scale change corrections on $\omega_{a}$, the correction (Eq. 4.7) is scaled according to

$$
E\left(\alpha_{\mathrm{ESC}}\right)=\frac{E^{\prime}}{1+\alpha_{\mathrm{ESC}}(\xi(t)-1) / m}
$$




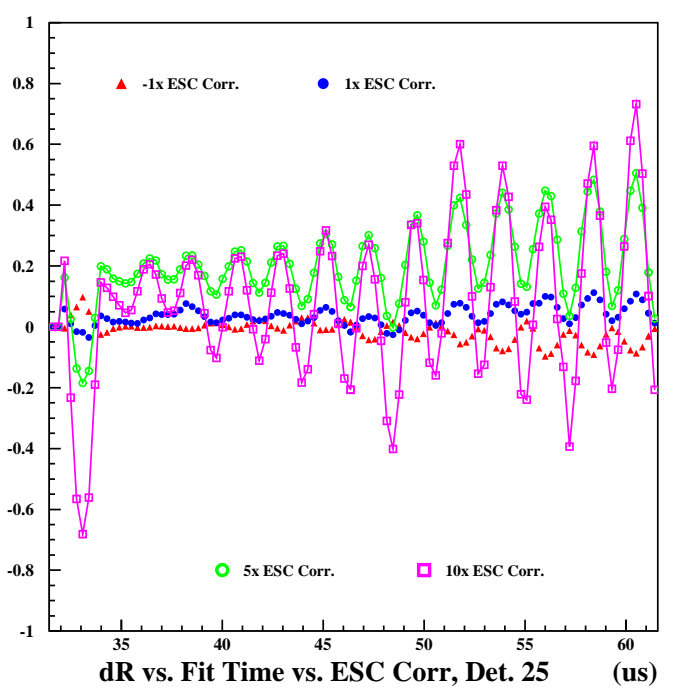

(a) Low-n data set.

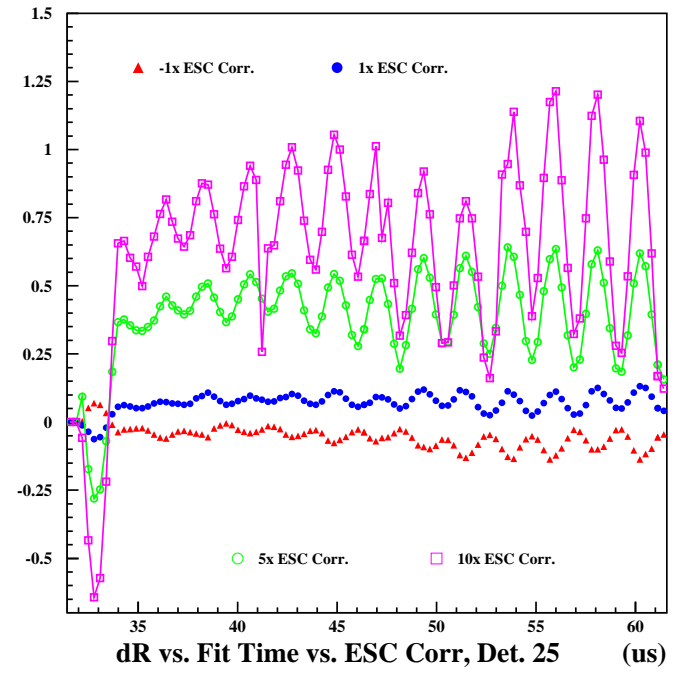

(b) High-n data set.

Figure 4.48: Difference of $R$ vs. fit start time plots for the sum of all detector data between uncorrected and gain-corrected data. Left: low-n results. Right: high-n results.

Therefore, if $\alpha_{\mathrm{ESC}}=0$, no correction is applied, whereas $\alpha_{\mathrm{ESC}}=1$ corresponds to the applied correction. Fig. 4.48 plots the difference in resulting $R$-values between uncorrected and gain-corrected data for the low- and high-n data sets. The solid blue circles represent $R\left(\alpha_{\mathrm{ESC}}=0\right)-R\left(\alpha_{\mathrm{ESC}}=1\right)$ vs. fit time, the solid red triangles represent $R\left(\alpha_{\mathrm{ESC}}=0\right)$ $R\left(\alpha_{\mathrm{ESC}}=-1\right)$, and the open green circles and open purple squares represent similar differences involving $R\left(\alpha_{\mathrm{ESC}}=5\right)$ and $R\left(\alpha_{\mathrm{ESC}}=10\right)$, respectively. The differences are small for reasonable gain corrections (eg, $\alpha_{\mathrm{ESC}} \simeq 1$ ): a maximum value of $0.08 \mathrm{ppm}$ for the low-n data set and a maximum value $0.15 \mathrm{ppm}$ for the high-n data set. The difference also oscillates at twice the $g$-2 frequency, which is expected from the ratio method (see Appendix B.2). The large differences seen when a $\alpha_{\mathrm{ESC}} \geq 5$ correction is applied is a statistical effect. Large corrections to the energies result in large changes the number of events, such that many high energy electrons are thrown out due to the applied upper energy cut, and many lower energy electrons are added to the time spectrum that otherwise would not have passed the applied lower energy cut.

To determine the systematic error on $R$ from energy-scale change corrections, the slope $\delta R / d \alpha_{\mathrm{ESC}}$ is determined from fits to the data using the nine parameter ratio function at each detector's fit start time. As an example, the plot on the left of Fig. 4.49 shows $R$ vs. $\alpha_{\mathrm{ESC}}$ for detector 2 , using the low-n data set. The plot is fit to a straight line to obtain the slope $\delta R / d \alpha_{\mathrm{ESC}}$ in units of $\mathrm{ppm} / \alpha_{\mathrm{ESC}}$. The systematic uncertainty on $R$ is therefore

$$
\delta R_{\mathrm{ESC}}=\frac{d R}{d \alpha_{\mathrm{ESC}}} \times \delta \alpha_{\mathrm{ESC}}
$$




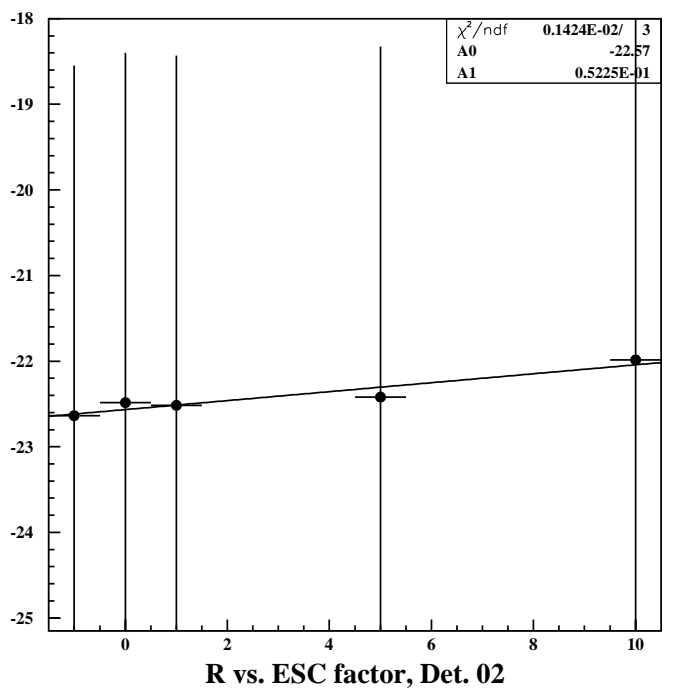

(a) $R$ vs. $\alpha_{\mathrm{ESC}}$ for detector 2 (low-n data set).
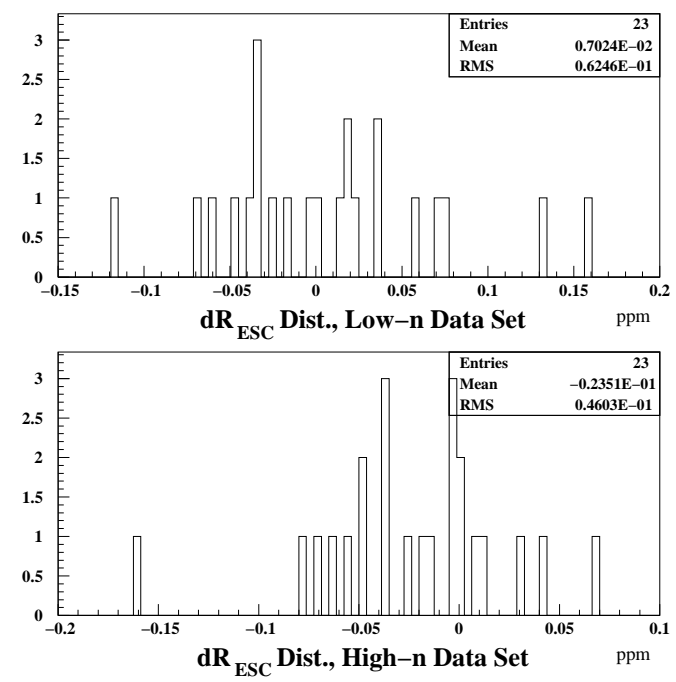

(b) Distributions of $\delta R_{\mathrm{ESC}}$ over detectors.

Figure 4.49: $\delta R_{\mathrm{ESC}}$ is determined from the slopes $\delta R / d \alpha_{\mathrm{ESC}}$ for each detector and the conservative estimate $\alpha=1 \pm 0.4$ in the applied correction.

where $\delta \alpha_{\mathrm{ESC}}$ is the uncertainty of the applied correction. A comparison of the various energy scale change corrections applied by the $\omega_{a}$ analyzers shows agreement to within $40 \%$, which is taken as the uncertainty of the applied correction. The plots on the right of Fig. 4.49 are the distributions of $\delta R_{\mathrm{ESC}}$ over the values determined for the 23 detectors for the two data sets using the above prescription. We find that $\delta R_{\mathrm{ESC}}$ is less than $0.01 \mathrm{ppm}$ for the low-n data set, and $0.024 \mathrm{ppm}$ for the high-n data set.

As discussed in Section 4.2.5, another energy-scale systematic effect is the correlation between reconstructed energy and island length. I. Logashenko calculated the effect of island length on average energy and estimated the energy-scale correction as a function of time for each detector. Fig. 4.50(a) shows the correction for the worst-affected detector (7) and a typical "quiet" detector (16). There is a $g$-2 oscillation in the correction since there is a a higher probability of having two or more pulses on a WFD island at the peak of the $g$ - 2 cycle than at the trough. However, the particular shape and magnitude of the corrections are difficult to predict, since they depend on the shape of the flash background seen by each detector. Those detectors closest to and downstream from the point of injection obviously see the largest effect, since they see the largest amount of flash. However, for some unknown reason, detector 7 behaves worse than detector 4; the magnitude of the correction is nearly twice as large for detector 7 , yet the flash background is nearly half that seen in detector 4 . Luckily the amplitude of the oscillations is fairly small, at the level of $10^{-4}$. The differences in $R$ as a function of time for detectors 7, 16 and 25 (the sum of all detectors) are shown in Fig. 4.50(b). As expected, detector 7 shows the largest difference in $R$. The effect on the 


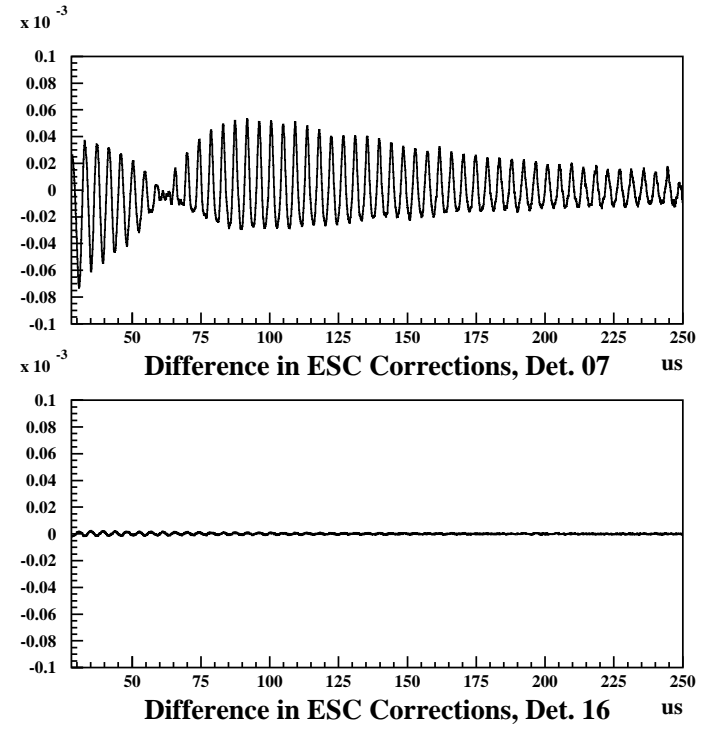

(a) Differences in the ESC correction from the pulse fitting algorithm's dependence on island length for detectors 7 and 16.

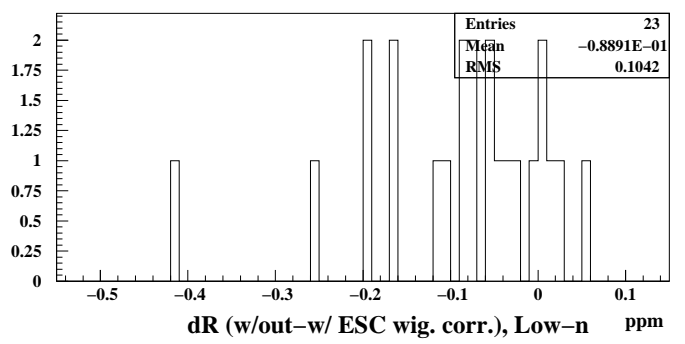

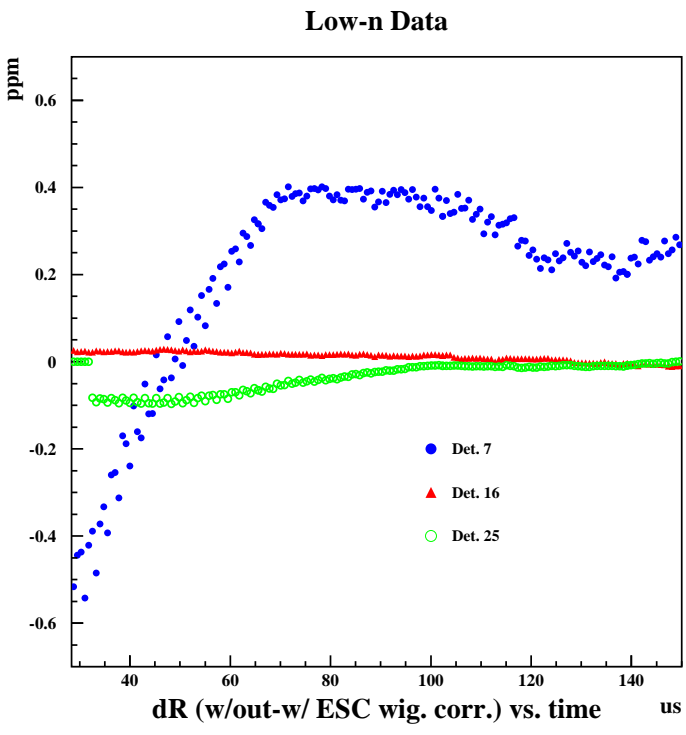

(b) $\delta R$ versus time for detectors 7,16 and 25 , where $\delta R$ is the difference in $R$ found between applying and not applying the island length ESC correction to the data.

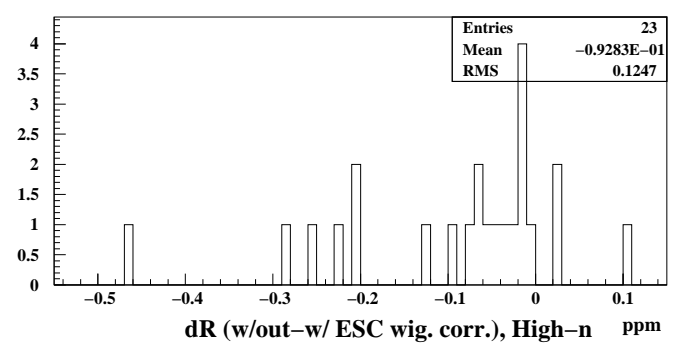

(c) $\delta R$ distributions for both the low- and high-n data sets at each detectors fit start time.

Figure 4.50: The varying WFD island length results in another ESC-like systematic uncertainty on $\omega_{a}$. The island length varies with the $g$-2 frequency since there is a higher probability of having two or more pulses on an island at the peak of the $g-2$ cycle than at the trough. 
sum of all detectors is found to be approximately $0.1 \mathrm{ppm}$ near $32 \mu \mathrm{s}$ after injection.

The two plots in Fig. 4.50(c) show the distributions of the differences in $R$ between fits to corrected and uncorrected data for each detector at their individual start times. The results are very consistent for both data sets: the mean difference in $R$ is $\sim 0.09 \mathrm{ppm}$. We therefore claim $0.1 \mathrm{ppm}$ as the systematic uncertainty due to this effect. Note that we do not yet add this in quadrature to the systematic uncertainty on $R$ from the applied energy-scale corrections, since we wish to treat these two effects differently when combined with other systematic errors (eg: pileup subtraction).

\subsubsection{Coherent Betatron Oscillations}

With the nine parameter physics function, the CBO systematic error on $R$ arises primarily from uncertainties in the frequency and functional form of the CBO. To determine the CBO systematic error on $R$, the slopes $d R / d \tau_{c}$ and $d R / d f_{c}$ are determined from fits to the nine parameter ratio function. For the low-n data set, the CBO lifetime was varied from 82.1 to $102.1 \mu \mathrm{s}$ in $5 \mu \mathrm{s}$ steps, and in a separate study, the CBO frequency was varied from 417.5$420.7 \mathrm{kHz}$ in $0.8 \mathrm{kHz}$ steps. Similarly, for the high-n data set, the CBO lifetime was varied from 120.1 to $140.1 \mu \mathrm{s}$ in $5 \mu$ s steps and the CBO frequency was varied from $489-492.2 \mathrm{kHz}$ $0.8 \mathrm{kHz}$ steps. The uncertainties in $R$ are then given by

$$
\delta R_{\tau_{c}}=\delta \tau_{c} \times \frac{d R}{d \tau_{c}}, \quad \delta R_{f_{c}}=\delta f_{c} \times \frac{d R}{d f_{c}}
$$

where $\delta \tau_{c}$ and $\delta f_{c}$ are the uncertainties of the CBO lifetime and frequency. The slopes $d R / d \tau_{c}$ and $d R / d f_{c}$ are determined for each detector by fitting a straight line to the $R$ vs. $\tau_{c}$ and $R$ vs. $f_{c}$ plots, and the distributions of these slopes for the 23 detectors are shown in Fig. 4.51. Taking the mean values of the distributions, and an uncertainty of $5 \mu \mathrm{s}$ in the $\mathrm{CBO}$ lifetime and $0.6 \mathrm{kHz}$ in the $\mathrm{CBO}$ frequency, we find for the low-n data set a value of $d R_{\tau_{c}}=0.01 \mathrm{ppm}$ and $d R_{f_{c}}=0.03 \mathrm{ppm}$. For the high n-value data set the situation is a bit worse, where we find $d R_{\tau_{c}}=0.02 \mathrm{ppm}$ and $d R_{f_{c}}=0.07 \mathrm{ppm}$. Assuming, quite conservatively, that the CBO frequency and lifetime are $100 \%$ correlated, these errors will be added linearly.

In addition to the intrinsic uncertainty of the CBO frequency, we must also consider the effect of having a time dependent CBO frequency. Since the scraping voltage is turned off (that is, increased to the nominal voltage) with an $\mathrm{RC}$ time constant of $5 \mu \mathrm{s}$ at $7 \mu \mathrm{s}$ after injection, the quadrupole voltages are still slightly changing at our earliest fit start times. During scraping, the n-value is [95]

$$
n_{1}=\frac{V_{1}+V_{0}}{2 V_{0}} n_{0}
$$

where the subscript " 1 " refers to the value during scraping and the subscript " 0 " refers to the nominal value (eg: after scraping). The quadrupole voltages during scraping are set to $70 \%$ their nominal value, so $n_{1}=0.85 n_{0}$. Therefore, we have

$$
n(t)=n_{0}\left(1-0.15 e^{-(t-7) / 5}\right), t \geq 7
$$



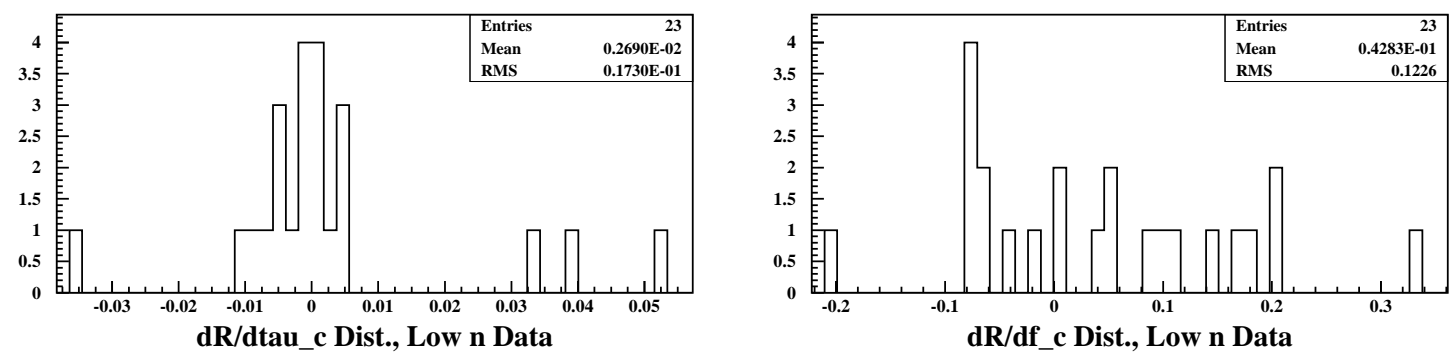

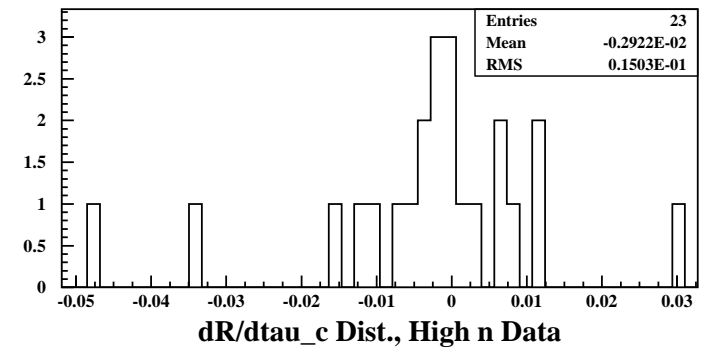

(a) Distribution of $d R / d \tau_{c}$ values.

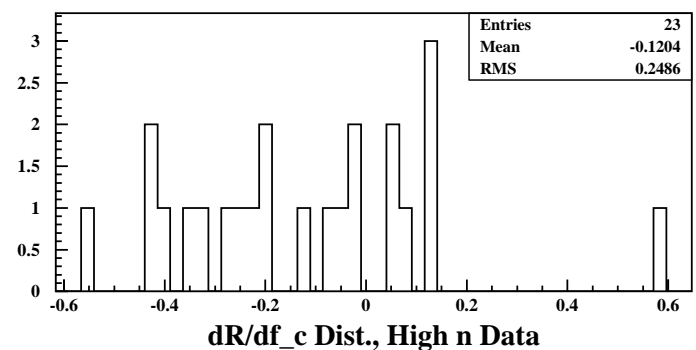

(b) Distribution of $d R / d f_{c}$ values.

Figure 4.51: Distributions of CBO uncertainties determined for each of the 23 detectors at their corresponding fit start times. Top: Low-n data set. Bottom: High-n data set.

Since

$$
f_{c b o}=f_{c}(1-\sqrt{1-n})
$$

where $f_{c}$ is the cyclotron frequency, then plugging in the above time-dependent $\mathrm{n}$-value one can easily show that

$$
f_{\text {cbo }}(t)=f_{\text {cboo }_{0}}-\frac{0.15 f_{c} n_{0}}{2 \sqrt{1-n_{0}}} \exp (-(t-7) / 5)
$$

At $\sim 23 \mu$ s after injection, the CBO frequency is nearly $0.5 \%$ lower than its nominal value. However, putting this time-dependent frequency into the 9-parameter ratio fits did not improve the $\chi^{2}$ of the fits to the data at early times, and the distribution of differences in $R$ from fits with and without the time-dependent CBO frequency has a mean value of 0.02 ppm for both the low- and high-n data sets. We therefore claim $0.02 \mathrm{ppm}$ as the systematic uncertainty from the time-dependence of the $\mathrm{CBO}$ frequency.

Another issue to consider is whether or not all CBO terms have the same functional form. To estimate the sensitivity of $R$ to different different $\mathrm{CBO}$ envelopes for different CBO terms, the data were fit assuming different lifetimes of the $N_{c}(t)$ term than for the $A_{c}(t)$ and $\phi_{c}(t)$ terms. For the low-n data set, the average value (over fits to the 23 individual detectors) of $\partial R / \partial \tau_{N}$ is $2.2 \times 10^{-4} \mathrm{ppm} / \mu$ s and the average value of $\partial R / \partial \tau_{A, \phi}$ is $-4.2 \times 10^{-5}$ $\mathrm{ppm} / \mu \mathrm{s}$. For the high-n data set, $\partial R / \partial \tau_{N}=7.6 \times 10^{-5} \mathrm{ppm} / \mu$ s and $\partial R / \partial \tau_{A, \phi}=-1.6 \times 10^{-3}$ $\mathrm{ppm} / \mu \mathrm{s}$. Since the RMS spread of the distributions of CBO lifetimes determined from the studies in Section 4.3.1 is approximately $50 \mu \mathrm{s}$, this is a reasonable estimate of the spread 
in lifetimes that would be representative of a very different CBO functional form and/or lifetime of the CBO. Therefore, we find the uncertainty in $R$ from the uncertainty in the CBO envelope is $0.01 \mathrm{ppm}$ for the low-n data set and $0.08 \mathrm{ppm}$ for the high-n data.

Finally, it is possible that the functional form of the $\phi_{c}(t)$ term has two contributions, one from the time dependence of the radial mean of the beam and the other from the time dependence of the radial width of of the beam. Each contribution may have a different lifetime and phase. The absolute worst-case scenario is if the mean and width contributions have completely opposite phases. Although we know from tracking simulations that the width and mean are only slightly out of phase, the worst-case-scenario was studied in fits to computer generated time spectra. Twenty-four time spectra were produced with $N_{c}, A_{c}$ and $\phi_{c}$ terms included with different phases that vary linearly (from 0 to $2 \pi$ ) around the ring, and equal amplitudes for each spectra except three (eg: the kicker detectors), where the amplitudes are $50 \%$ larger. The general form for the $\phi_{c}(t)$ term is

$$
\phi_{c}(t)=\phi_{c_{0}}\left(A_{1} \exp \left(-t / \tau_{c_{1}}\right) \cos \left(\omega_{c} t+\phi_{c}\right)-\left(1-A_{1}\right) \exp \left(-t / \tau_{c_{2}}\right) \cos \left(\omega_{c} t+\phi_{c}\right)\right)
$$

The relative amount of mixing between the radial mean contribution and the radial width contribution to $\phi_{c}(t)$ was varied such that $A_{1}=15-75 \%$ in $15 \%$ steps. Furthermore, $\tau_{c_{2}}$ was varied from $0.5 \times \tau_{c_{1}}$ to $1.5 \times \tau_{c_{1}}$ in three steps. On average, the $R$ value obtained from the naive 9-parameter fit differed by $0.04 \mathrm{ppm}$ from the input value, with a spread of $0.04 \mathrm{ppm}$. We therefore claim $0.04 \mathrm{ppm}$ as our estimate of the systematic uncertainty due to the CBO phase term having out-of-phase contributions from the radial mean and radial width.

Adding all uncertainties together in quadrature, the total CBO systematic error is 0.06 and $0.13 \mathrm{ppm}$ for the low-n and high-n data sets, respectively.

\subsubsection{Pileup}

Pileup is a time dependent background that oscillates at the $g$-2 frequency. Therefore, to estimate the pileup systematic uncertainty, one must estimate the efficiency with which the pileup time spectrum is constructed as well as the uncertainty on its phase. The pileup construction efficiency may be regarded as the uncertainty on the amplitude of the background.

The pileup amplitude systematic uncertainty on $R$ is determined from the relation

$$
d R_{\alpha_{P U S}}=\delta \alpha_{P U S} \times \frac{d R}{d \alpha_{P U S}}
$$

where $\delta \alpha_{P U S}$ is the uncertainty on the pileup amplitude. To determine $\delta \alpha_{P U S}$, we compare pileup-subtracted (PUS) energy spectrum of decay electrons with $20<t<250 \mu$ s and the unsubtracted energy spectrum of decay electrons with $t>250 \mu \mathrm{s}$. Since there is less data after $250 \mu \mathrm{s}$, the energy spectra from the late-time data must be scaled up by a factor $\lambda$. The scale factor $\lambda$ is obtained by taking the ratio of $N_{\text {early }}(E)$ to $N_{\text {late }}(E)$ and fitting the region $2.3 \mathrm{GeV}<E<2.7 \mathrm{GeV}$ to a constant. Typical values for $\lambda$ are 34.3 , which is very close to what one would expect from the simple approximation of $\exp ((250-20) / 64.4$. 


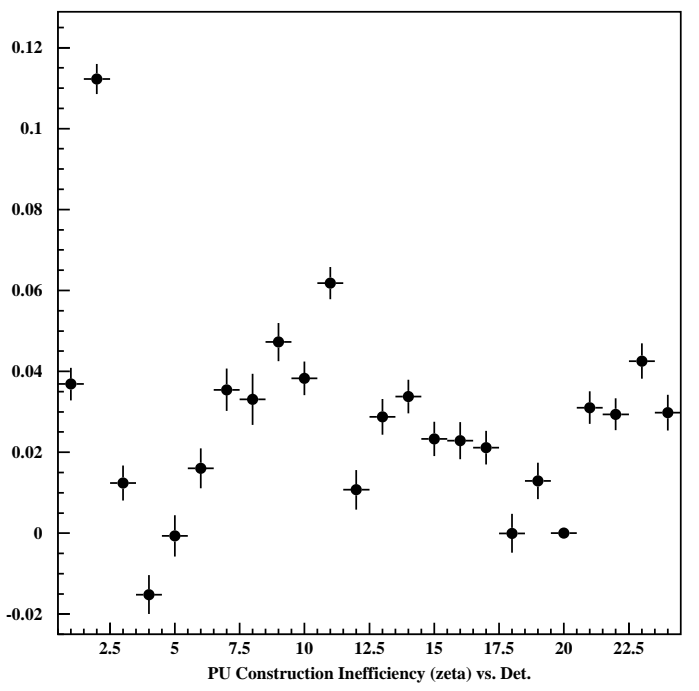

(a) Pileup construction efficiency (average $\zeta$ from 3.1-4.5 GeV) vs. detector.
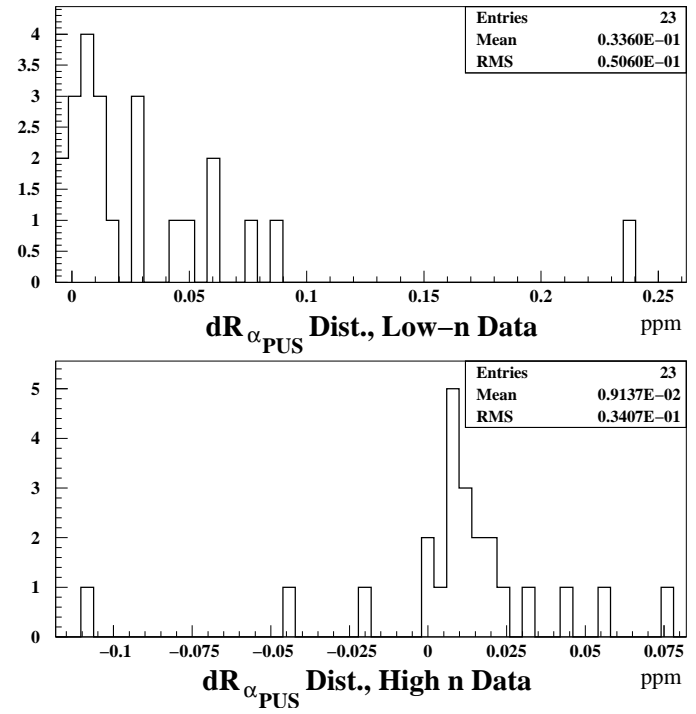

(b) Distributions of $d R_{\alpha_{P U}}$, the systematic error on $R$ due to pileup construction efficiency, for both data sets.

Figure 4.52: Estimation of $\delta R_{\alpha_{P U S}}$, the pileup construction efficiency systematic error.

We define the parameter $\zeta$ such that

$$
\zeta(E)=\frac{N_{\text {early }_{P U S}}(E)-\lambda N_{\text {late }}(E)}{N_{\text {early }}(E)-\lambda N_{\text {late }}(E)}
$$

Assuming no energy-dependence in constructing pileup pulses, the average value of $\zeta$ in the region where pileup dominates the spectrum (energies above $3.1 \mathrm{GeV}$ ) is an approximation of the inefficiency with which we construct the pileup, $\delta \alpha_{P U S}$. Fig. 4.52 (a) shows the average values of $\zeta$ versus detector, where the average value of $\zeta$ is taken from 3.1-4.5 GeV. With a few exceptions, we typically construct pileup with an efficiency $>95 \%$.

The slope $d R / d \alpha_{P U S}$ are determined from straight-line fits to $R$ vs. $\alpha_{P U S}$, where the $R$-values are obtained from fits to data with varying fractions, $\alpha_{P U S}$, of pileup subtracted. In this study, $\alpha_{P U S}$ ranged from 0.9-1.1 in 0.025 steps. Fig. 4.52(b) shows the distributions of $d R_{\alpha_{P U}}$, the systematic error on $R$, over the 23 detectors, for each data set. The values of $d R_{P U_{e}}$ were taken from the product of $d R / d \alpha_{P U S}$ and the inefficiency $(\delta \alpha)$ of pileup construction determined for each detector. Taking the means of these distributions as the average systematic error, we find that $d R_{P U_{e}}=0.034 \mathrm{ppm}$ for the low n-value data set, and $0.009 \mathrm{ppm}$ for the high n-value data set.

As has been discussed[96], there could be a difference between constructed and true pileup phases. To investigate this effect, decay electron time spectra were generated assum- 


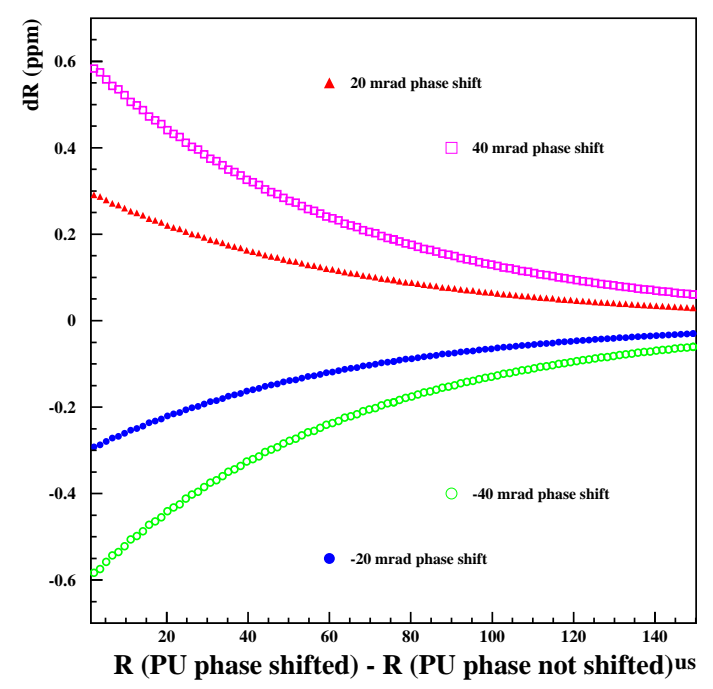

(a)

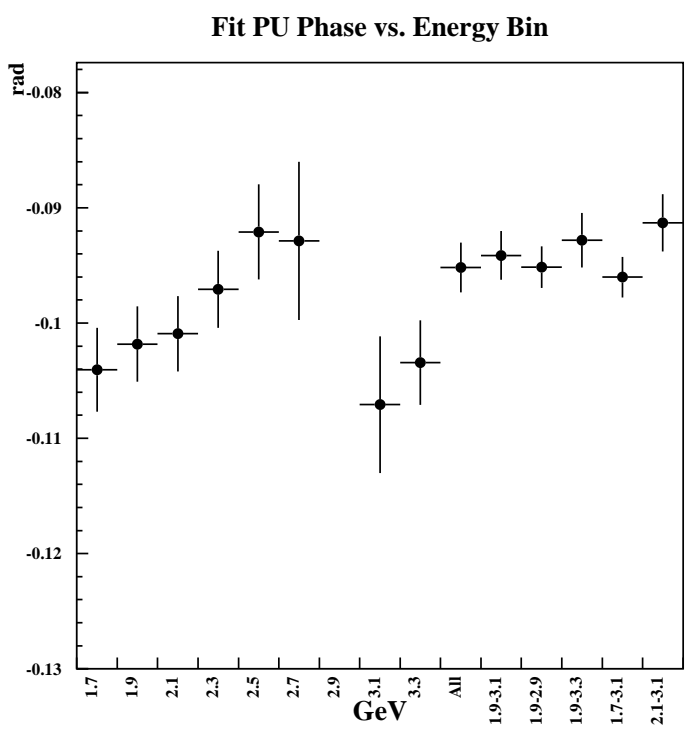

(b)

Figure 4.53: The dependence $d R / d \phi_{P U}$ is determined from fits to simulated decay electron time spectra with a difference in phase between the true pileup and constructed (and subtracted) pileup. $d R$ vs. fit start time are plotted in Fig. 4.53(a) for phase differences of -40 , $-20,20$, and 40 mrad. The simulated time spectra assume a $0.42 \%$ pileup contamination at the fit start time. Fig. 4.53(b) shows the fitted pileup phase as a function of energy. The phases are determined by fitting the energy-binned pileup time spectra to Eq. 4.55.

ing the five parameter function with a pileup term of the form

$$
N_{\mathrm{PU}}(t)=N_{0_{\mathrm{PU}}} e^{-2 t / \tau_{\mu}}\left(\cos \left(\omega_{a} t+\phi_{\mathrm{PU}}+A_{1} \cos \left(2 \omega_{a} t+\phi_{\mathrm{PU}_{2}}\right)+A_{2}\right)\right.
$$

The function has terms that go as rate $\left(\cos \left(\omega_{a} t\right)\right)$ and rate-squared $\left(\cos ^{2}\left(\omega_{a} t\right) \sim \cos \left(2 \omega_{a} t\right)\right)$, and a constant term as well. The values $N_{0_{\mathrm{PU}}} / N_{0}=0.42 \%, A_{1}=0.07$ and $A_{2}=0.34$ used to generate the time spectra were obtained from fits to data-constructed pileup, as were the phases $\phi_{P U}=3.0474, \phi_{P U_{2}}=2.9584$. The pileup background term with different phases than those used to create the original time spectrum was then subtracted out. Differences between "true" and "constructed" phases were varied from -40 to $40 \mathrm{mrad}$, in $20 \mathrm{mrad}$ steps. The plots shown in Fig. 4.53(a) show differences in fit values of $R$ as a function of fit time between spectra that have the "true" pileup subtracted off and time spectra that have phase-shifted pileup subtracted off. An error in the phase of pileup construction of 20 mrad results in a $\sim 0.3 \mathrm{ppm}$ error in $\mathrm{R}$.

The dominant error in the constructed pileup phase arises from uncertainties in either the Logashenko coefficients or the energy-dependent dead-time used to construct the pileup pulses. Pulses will be added or lost "near" the edges of the upper and lower energy thresholds if the energies of the pileup pulses are mis-constructed. Since the phase is energy-dependent, 
this could cause the constructed pileup phase to differ from the true pileup phase. According to Logashenko[97], the energy-dependent dead-time and coefficients used to construct the pileup have an uncertainty of $\sim 10 \%$.

To estimate the size of this effect, energy-binned pileup time spectra were fit to Eq. 4.55; the fit values of the pileup phase vs. energy bin are shown in Fig. 4.53. The energy bins are $200 \mathrm{MeV}$ wide, and results from energy bins 1.7-3.3 GeV are shown. In addition, fits to larger energy bins were made: "All" represents the sum over all energy bins, and the numbered bins represent the sum over those bins (eg: 1.9-3.1 GeV is the sum of the data from 1.8 to $3.2 \mathrm{GeV}$ ). Of the last five, the 1.9-3.1 bin is very similar to the energy-cuts used in constructing the time spectra for this analysis, and so the last four bins, 1.9-2.9, 1.9-3.3, 1.7-3.1 and 2.1-3.1 are as if $100 \%$ of the bins on the edges of the energy-cuts were added in or subtracted off from the data. We find that the largest change $(\sim 2.8 \mathrm{mrad})$ in pileup phase arises when the lowest energy bin is thrown out. This is not unexpected, since the pileup phase changes more rapidly in this energy region than in the higher energy region. Although it is difficult to imagine that an entire $200 \mathrm{MeV}$-wide energy bin could have been during pileup construction, we nevertheless use this very conservative estimate as an upper limit on the systematic error of the constructed pileup phase. From Fig. 4.53 we see that a $2.8 \mathrm{mrad}$ uncertainty in the PU phase results in a $0.042 \mathrm{ppm}$ systematic error on $R$.

Very low-energy pulses that sit below the pedestal in the WFD data stream are not reconstructed and are therefore "invisible". Since there are a great number of such pulses, we must concern ourselves with how the effect the reconstruction of single pulses. The properties of the pulse reconstruction algorithm are such that on average the effect of lowenergy pulses on the fitted energy and time of single pulses cancels out. However, these pulses do effect the average observed phase. This was also studied by Logashenko[98] as well as G. Onderwater. Both found the same overall phase difference produced by the low energy pileup, however Logashenko's estimate of $0.06 \mathrm{ppm}$ was obtained assuming that a phase difference in the pileup of $1 \mathrm{mrad}$ is equivalent to a $3.5 \mathrm{ppm}$ error in $R$. Onderwater, on the other hand, did an actual simulation and found the sensitivity to be much smaller, by a factor of 3 . Since both studies found the same phase difference, Onderwater's estimate of $0.02 \mathrm{ppm}$ supersedes Logashenko's estimate of $0.06 \mathrm{ppm}$.

Adding all errors in quadrature gives $d R_{P U S}=0.06 \mathrm{ppm}$ for the low-n data set and 0.05 ppm for the high-n data set.

\subsubsection{Muon Losses}

To obtain the muon loss systematic error on $R$, we generate decay electron time spectra with no statistical fluctuations according to the five parameter functional form and include muon losses of varying amounts. The functional form of the muon losses is an empirical fit to the low-n 3FC time spectrum (see Section 4.2.7), where the data were fit to an exponential from 30-70 $\mu \mathrm{s}$, another exponential from 70-100 $\mu \mathrm{s}$, and a Gaussian from 100-300 $\mu \mathrm{s}$. The muon lifetime was also divided out of this function. Figs. 4.54 show the muon loss rate (left) and integrated muon losses (right). The integrated muon losses are normalized such that at very late times, the function goes to one. Therefore, a scale factor multiplying the integrated muon losses corresponds to the total (percentage of) muons lost during data taking. 


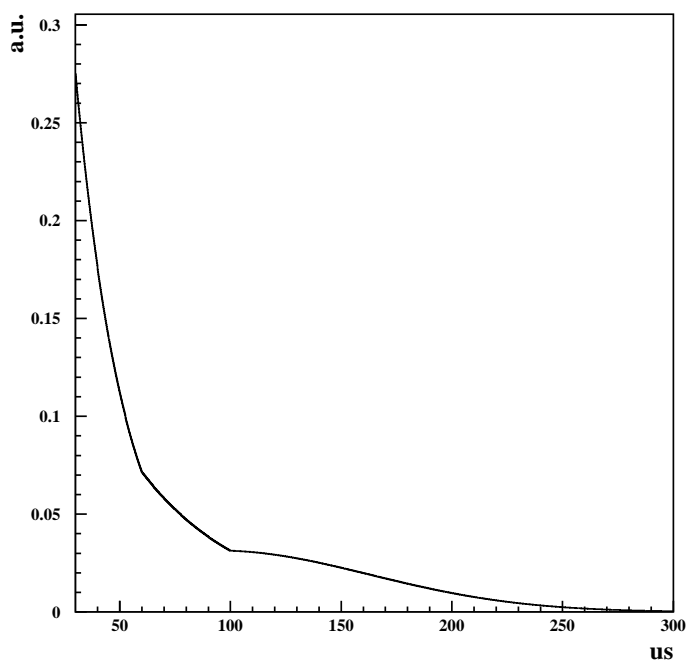

(a) Empirical fit to the low-n 3FC time spectrum with the muon lifetime divided out.

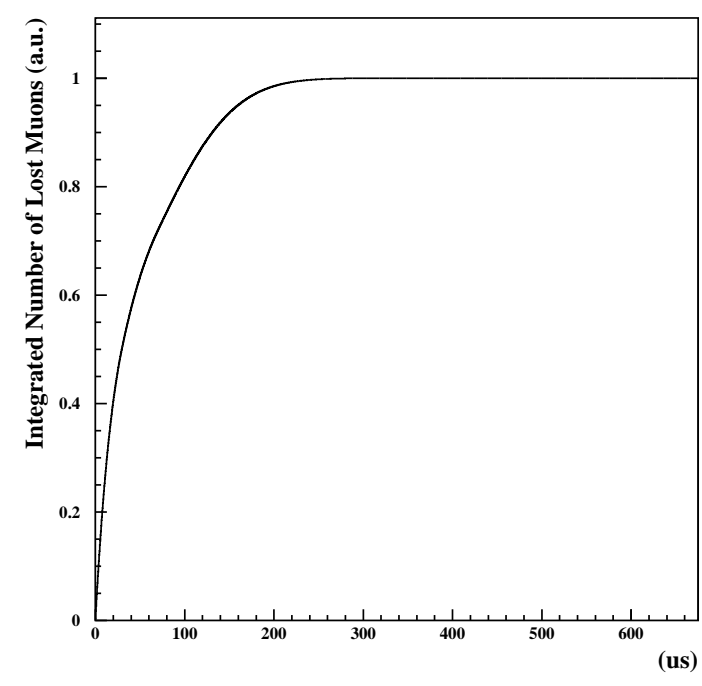

(b) Integrated muon losses, $F_{L}(t)$

Figure 4.54: An empirical description of the muon loss time spectrum is used to as a background in generated decay electron time spectra. The effect on $R$ is then determined from fits to these spectra.

The function used to create the time spectrum is

$$
N(t)=N_{0} e^{-t / \tau}\left(1+A \cos \left(\omega_{a} t+\phi\right)\right) \exp \left(-A_{L} F_{L}(t)\right)
$$

where

$$
F_{L}(t)=\int_{t_{0}}^{t} f_{3 \mathrm{FC}}\left(t^{\prime}\right) d t^{\prime}
$$

and $A_{L}=0.0,0.25 \%, 0.5 \%, 0.75 \%$ and $1.0 \%$. The ratio is then created from the original time spectrum and fit to the three parameter ratio function. Comparing the fit results of spectra with muon losses to the fit results of the spectrum with no muon losses, we find that the effect on $\mathrm{R}$ is extremely small. Fig. 4.55 shows the difference between fit results of spectra with and without muon losses. We see that if we lose a total of $0.6 \%$ of our injected muons after scraping, a number which is similar to loss rates determined from multi-parameter fits of the actual data, the effect on $\mathrm{R}$ is much less than $0.01 \mathrm{ppm}$ at the earliest fit times.

We should also consider whether the missing decay electrons from lost muons have a different average $g-2$ phase or asymmetry from the rest of the ensemble. To study the effect on $R$, time spectra were generated as described above, but with different asymmetries and $g-2$ phases for the lost decay electrons. The two plots in Fig. 4.56 show the size of the effect on $\omega_{a}$ for each of these effects. The effect of a slightly changing asymmetry (left plot) has very little effect on $\mathrm{R}$. This is no surprise, as we know that $A$ is not strongly correlated to $R$ in the fits. Indeed, most of the error on $\mathrm{R}$ is due to the effect on the number of decay 


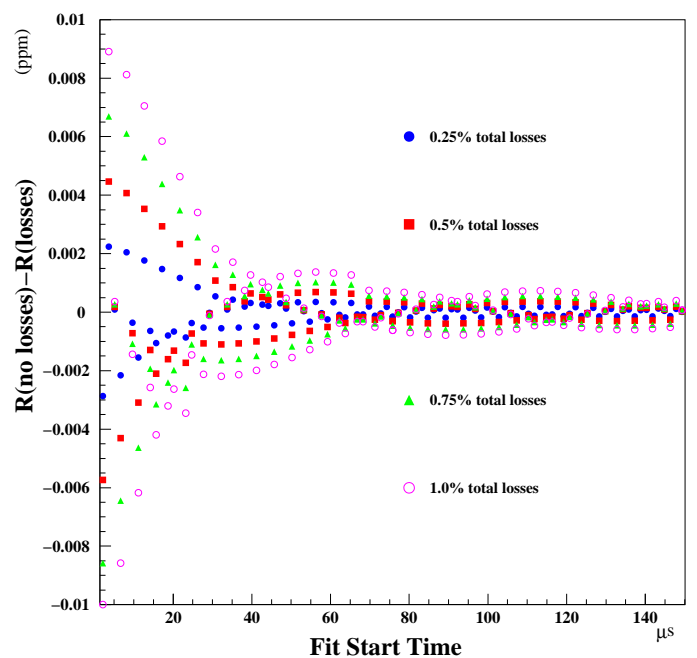

Figure 4.55: Effect of muon losses on $\mathrm{R}$ vs. fit start time with the ratio method. Plotted here is fit $\mathrm{R}$ (no losses) - fit $\mathrm{R}$ (losses). Note the scale of the effect: $<10^{-4} \mathrm{ppm}$.

positrons, since the amplitude of the phase pulling changes very slightly as the asymmetry changes.

However, as expected, a changing average $g$-2 phase has a much larger effect on $R$ (left plot of Fig. 4.56). A difference in $g-2$ phase between the stored and lost muons could arise from either beamline or storage ring beam dynamics. In the former, muons born before the pion momentum selection bend in the beamline (see Fig. 2.2) have a different $g$-2 phase than those muons born after the bend (in the straight pion decay channel of the beamline). In the latter, lost muons typically exist at the edges of the phase-space distribution, and correlations between the observed $g$-2 phase and position the muon when it decays.

\section{Beamline Effects}

As muons pass the dipole magnets used for pion momentum selection, their spins are rotated by $\sim 13 \mathrm{mrad}[99]$. However, most muons that are stored in the $g$ - 2 ring are born after this point in the beamline. Since muons born before and after this point in the beamline have different momenta, the muons born earlier may be preferentially lost, resulting in a changing average $g$-2 phase during a fill.

The beamline lost muon systematic uncertainty on $R$ has been estimated using both beamline acceptance calculations and detailed tracking simulations. W. Morse uses the acceptance of the beamline and storage ring to set an upper limit of $d R_{\text {loss }}<0.13 \mathrm{ppm}$. The author used the g2track simulation. In order to obtain reasonable statistics, muons lost during scraping are used in this study. However, only muons that are lost after 4 turns around the storage ring are considered, which means these muons at least survive injection and the kick. Stored muons are those that have not been lost after $\simeq 75 \mu$ s after injection (muons do not decay in g2track).

The initial spins are obtained from the BTRAF program, which not only simulates the $g-2$ 


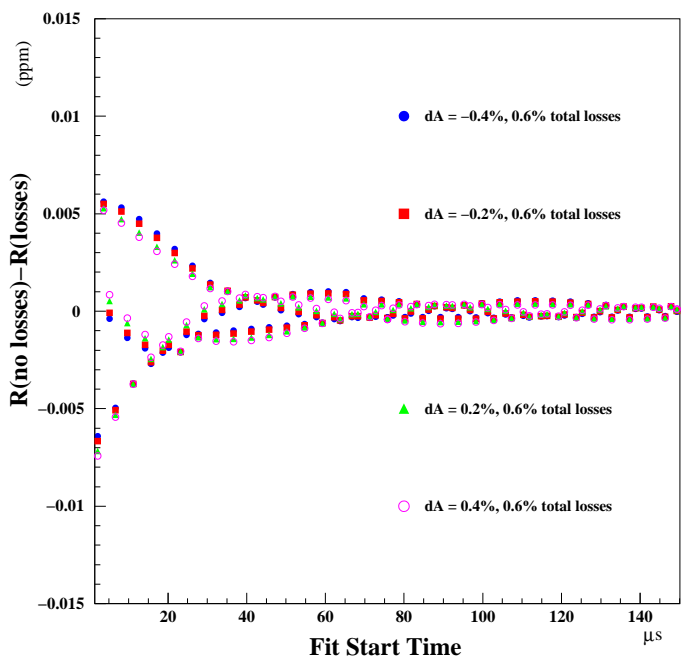

(a) Effect of lost muons having a different average asymmetry.

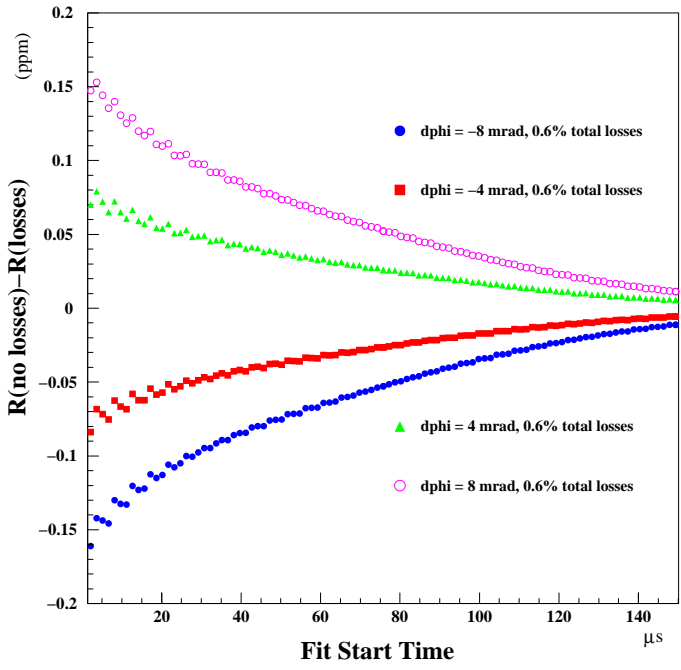

(b) Effect of lost muons having a different average $g$ - 2 phase.

Figure 4.56: Effect on $R$ vs. fit start time of muon losses assuming a $0.6 \%$ total loss and different average $g-2$ phases and asymmetries. The nominal average $g-2$ phase in the simulated spectrum is $1.5 \mathrm{rad}$, and that of the asymmetry is 0.4 .
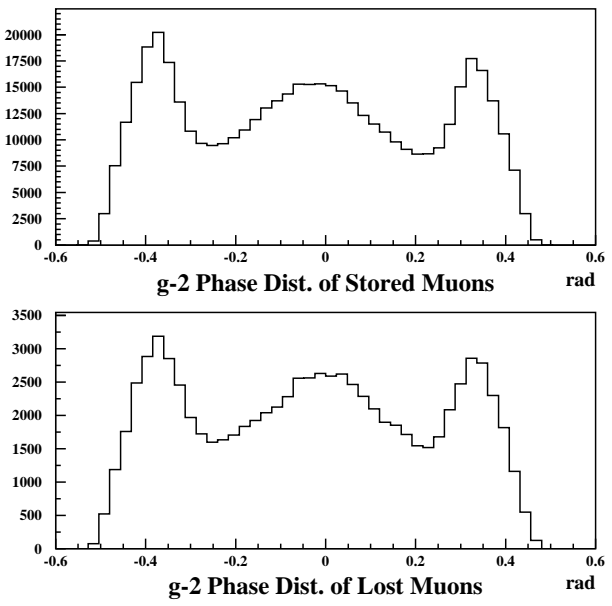

(a) Initial $g$-2 phases of the stored and lost muons found in g2track.

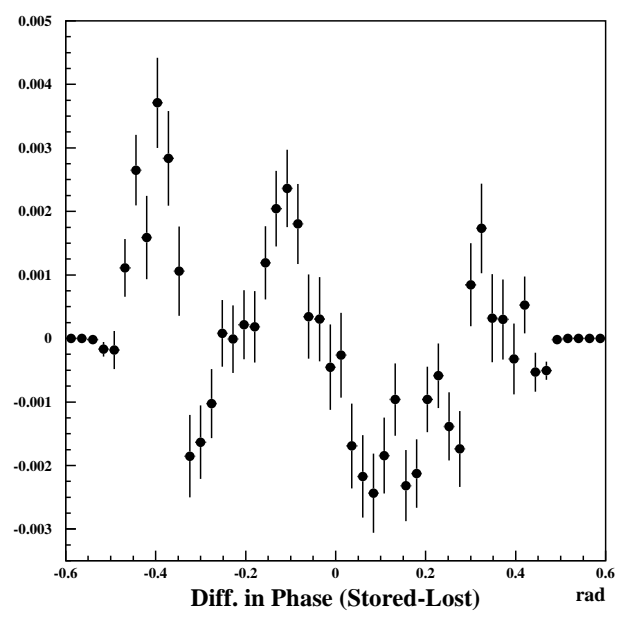

(b) Difference between the normalized distributions on the left.

Figure 4.57: Comparison of the distributions of the initial $g-2$ phases of stored (left) muons vs. those that are lost (right) in the g2track simulation. 


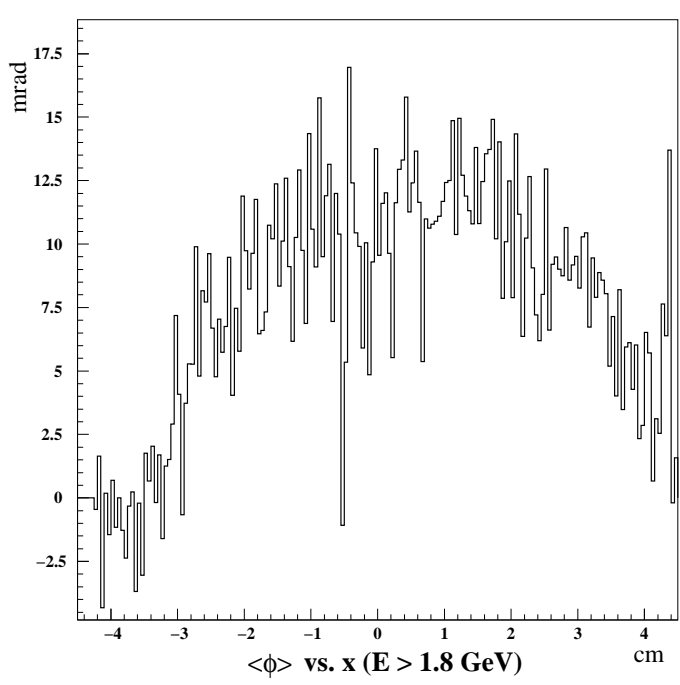

(a) $\langle\phi\rangle$ vs. radial position in the storage aperture at the point of decay.

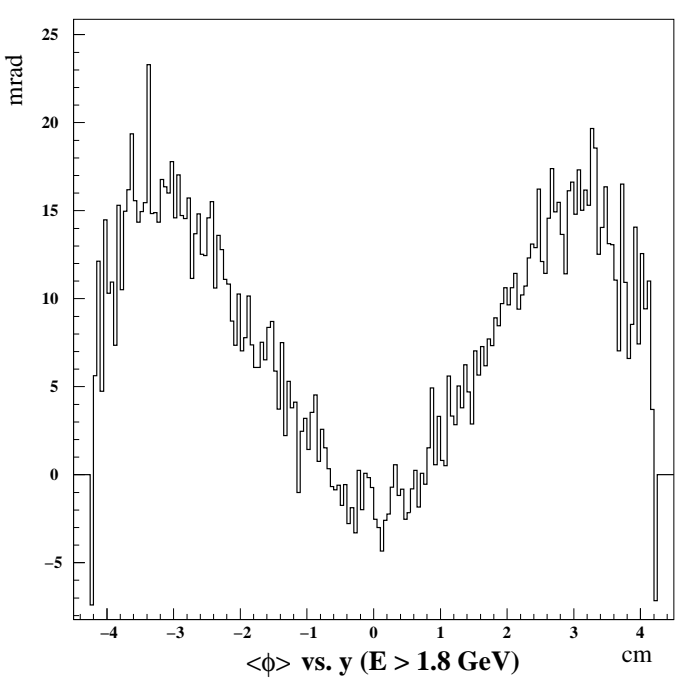

(b) $\langle\phi\rangle$ vs. vertical position in the storage aperture at the point of decay.

Figure 4.58: Average $g-2$ phase as a function of radial and vertical position in the storage aperture, determined from G2GEANT. The phase is averaged over azimuthal position (23 detectors), time and energies above $1.8 \mathrm{GeV}$.

beamline, but also tracks the spin of the muons born along the way from the AGS target to the end of the $g-2$ inflector. The $g-2$ phase of the muons at the time of injection is

$$
\phi_{x z}=\tan ^{-1}\left(\frac{s_{x}}{s_{z}}\right)
$$

The distributions of initial phases are shown in the right plots of Fig. 4.57. Using the $R M S / \sqrt{N}$ as the statistical error, we find that the average $g$-2 phase of the stored muons is $-35.5 \pm 0.4 \mathrm{mrad}$ and that of the lost muons is $-28.8 \pm 0.9 \mathrm{mrad}$. Therefore, there is a difference of $-6.7 \pm 1 \mathrm{mrad}$. Looking at Fig. 4.56 and interpolating between the -4 and -8 mrad plots, we see that this will give an approximate error of $0.1 \mathrm{ppm}$ for a total muon loss of $0.6 \%$. Since X. Huang finds that the total muon loss is approximately $0.5 \%$ for the low-n data set and $0.03-0.04 \%$ for the high-n data set, the error is scaled down to 0.08 and 0.06 ppm respectively at a fit start time of $30 \mu \mathrm{s}$.

\section{Storage Ring Beam Dynamics Effects}

The storage ring lost muon systematic uncertainty was also studied using g2track. The average $g$-2 phase was first determined as a function of position in the storage ring by Q. Peng and R. Carey using G2GEANT. Although $\phi(x, y)$ was determined in their studies, Fig. 4.58 shows only the projections along either the $y$ - or $x$-axes, $\phi(x)$ and $\phi(y)$. It is also important to note that the average phase was calculated from the sum of all data, integrated 
over both azimuthal position in the ring and in time.

Using the values of $\langle\phi(x, y)\rangle$, the average $g$-2 phase may be determined as a function of time in g2track. In this study, we sample as much of the phase space of the muon distribution as possible by assuming that each muon decays at the same azimuthal location in the ring at each turn around the ring. Figs. 4.59(a)-4.59(b) show the average phase as a function of time calculated in this manner for the two n-values used in the 2001 run. Note both the envelope and the amplitude of the CBO oscillations of $\langle\phi\rangle(t)$. The envelope does not appear to come from only either the radial mean vs. time or the radial width vs. time; it is more likely a mix of the two effects. The amplitude of the oscillations, on the order of $0.25 \mathrm{mrad}$, or nearly $175 \mathrm{ps}$, agrees very well with predictions for the drift-time effect. The reason the amplitude does not agree with the overall amplitude of the $\phi_{\text {cbo }}$ term obtained from fits is that the acceptance effect, which depends on the direction of the beam, was averaged out in the determination of $\langle\phi(x, y)\rangle$. Therefore, the only way we may directly see the full $\phi_{\text {cbo }}(t)$ effect is to produce $\langle\phi\rangle$ vs. $t$ plots from the G2GEANT data.

As mentioned previously, if the muons that are lost have a different phase-space distribution than muons that are not lost, then the average $g-2$ phase will change in time. To estimate this effect, we determine the average phase difference between lost and stored muons. Figs. 4.59(a)-4.59(b) show the average phase as a function of time for the stored muons. Figs. 4.59(c)-4.59(d) show the same for those muons that are (eventually) lost. We find that for the low-n lost muons, the average phase is larger (and increasing in time) than the stored muons, whereas for the high-n lost muons, the average phase is initially smaller than that of the stored muons, but also increases in time. The increase in the average phase arises from increases in the betatron amplitudes as the muons that will be lost undergo resonance.

We next generate time spectra as described above, however we assume a changing difference in phase between stored and lost muons. The time dependence of this difference is obtained from fits of a straight line to the data shown in Figs. 4.59(c) and 4.59(d). For the low-n data set,

$$
\Delta \phi(\text { lost }- \text { stored })=2.2 \mathrm{mrad}+t \times\left(2 \times 10^{-3} \mathrm{mrad} / \mu \mathrm{s}\right)
$$

and for the high-n data set,

$$
\Delta \phi(\text { lost }- \text { stored })=-2.2 \mathrm{mrad}+t \times\left(8 \times 10^{-3} \mathrm{mrad} / \mu \mathrm{s}\right)
$$

In the simulated time spectra, the slope $d \Delta \phi / d t$ was varied from 2.5 to $10.0 \times 10^{-3}$, and the growth of the lost muon phase was stopped at $100 \mu \mathrm{s}$. The level of losses assumed in the simulation is $0.5 \%$.

Converting the simulated time spectra to the ratio and using the three parameter ratio function, we find the (absolute) difference in $R$ (with respect to the value put into the simulation) to be between 0.03 and $0.044 \mathrm{ppm}$ for the low-n set at $30 \mu \mathrm{s}$. The effect is even smaller for the high-n set (see Fig. 4.60). However, because of uncertainties in the slope of the phase vs. time for the lost muons and the sign of phase, we conservatively estimate an upper limit of the systematic uncertainty: $d R<0.04 \mathrm{ppm}$. 


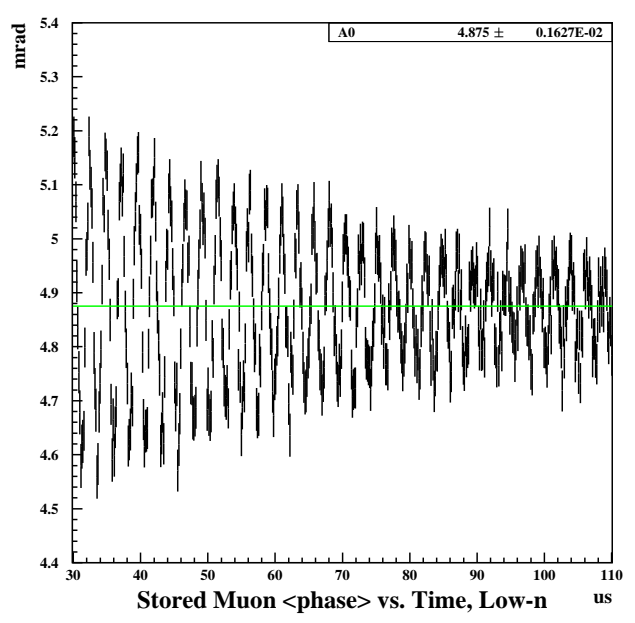

(a) $\langle\phi\rangle_{\text {stored }}$ vs. time for the low-n quadrupole setting.

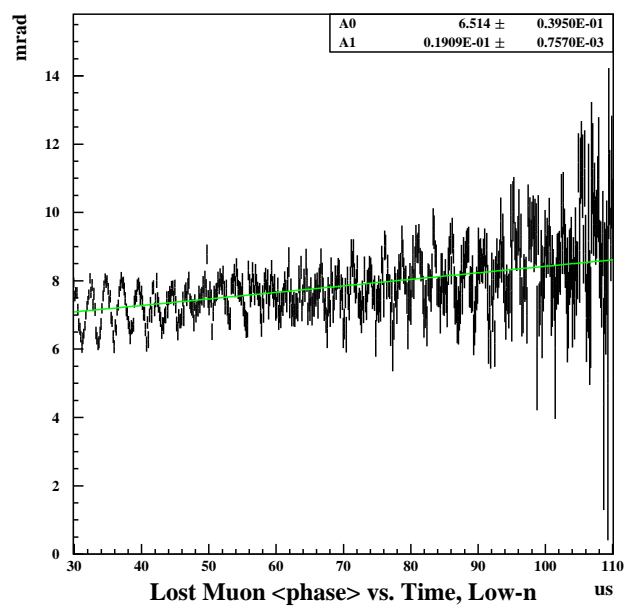

(c) $\langle\phi\rangle_{\text {lost }}$ vs. time for the low-n quadrupole setting.

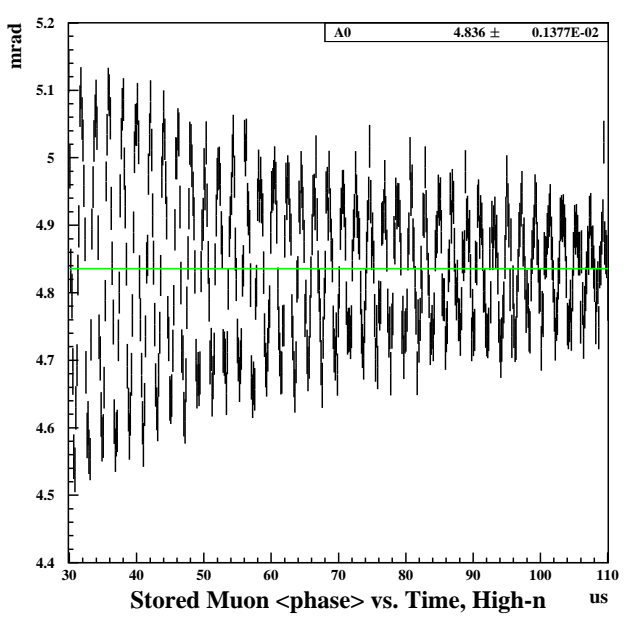

(b) $\langle\phi\rangle_{\text {stored }}$ vs. time for the high-n quadrupole setting.

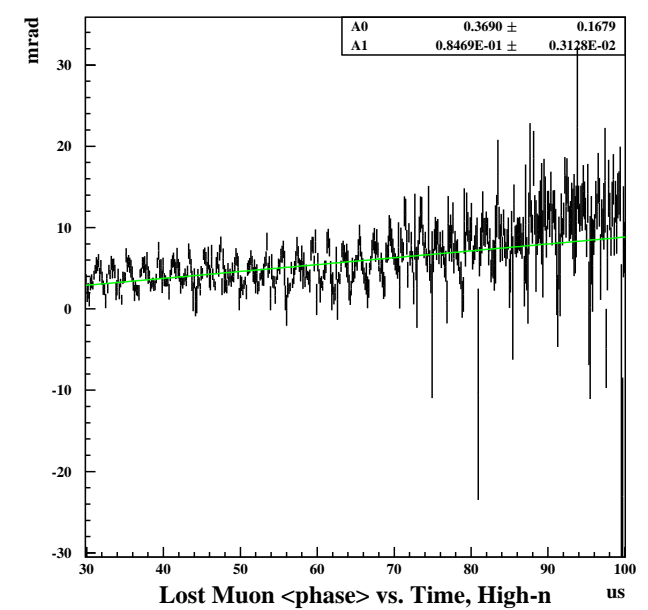

(d) $\langle\phi\rangle_{\text {lost }}$ vs. time for the high-n quadrupole setting.

Figure 4.59: Average $g$-2 phase of stored (top) and lost (bottom) muons in g2track as a function of time for the two quadrupole HV settings used in the 2001 run. 


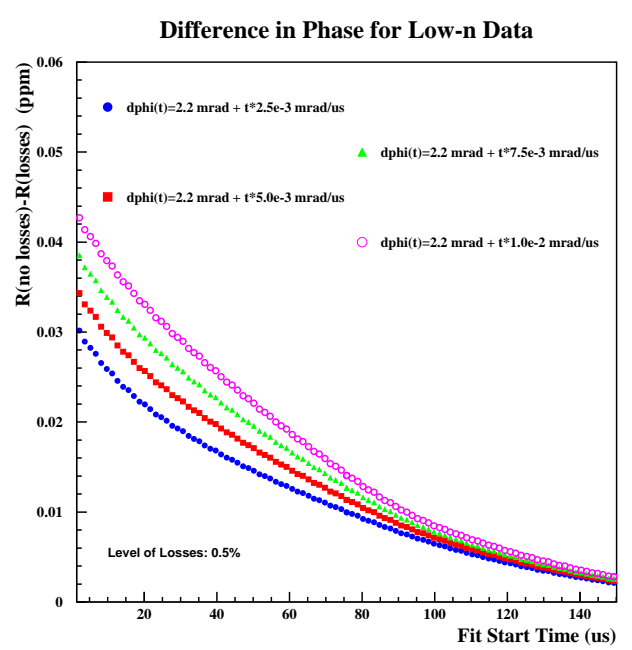

(a) $d R$ found for the difference found for the low-n quadrupole settings.

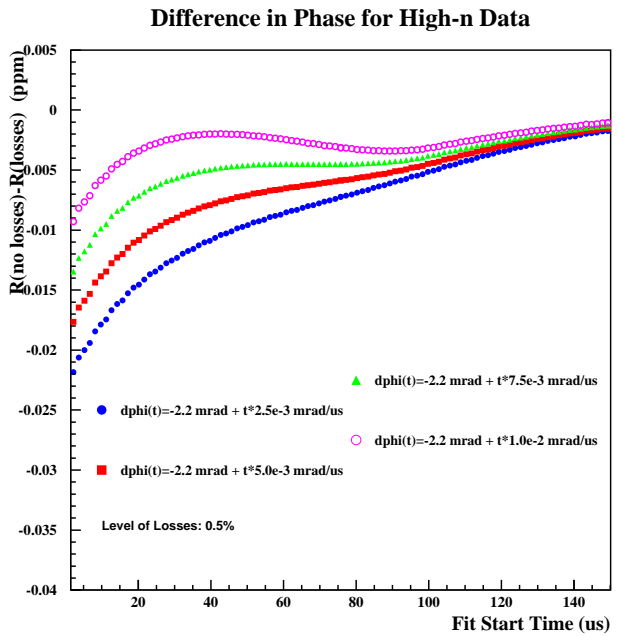

(b) $d R$ found for the difference found for the high-n quadrupole settings.

Figure 4.60: Changes in $R$ vs. fit start time for such differences between the lost and stored muon $g-2$ phases as were found from g2track simulations.

\section{Total Muon Loss Systematic Uncertainty}

The systematic uncertainties discussed here were calculated assuming a fit start time of $30 \mu \mathrm{s}$. Since in this analysis the average fit start time is $\sim 25 \mu \mathrm{s}$, we must scale up the error to account for the effect of starting our fits earlier by $5 \mu \mathrm{s}$. Extrapolating the muon loss function backward by $5 \mu \mathrm{s}$, we find that the total fraction of lost muons increases by $\sim 18 \%$. Therefore, for this analysis, the beamline lost muon systematic error is estimated to be $0.094 \mathrm{ppm}$ for the low-n data set and $0.071 \mathrm{ppm}$ for the high-n data set. The storage ring lost muon systematic is $0.047 \mathrm{ppm}$ for both data sets. Adding the uncertainties in quadrature, we find $d R_{\mu_{\text {loss }}}=0.11 \mathrm{ppm}$ for the low-n data set and $0.09 \mathrm{ppm}$ for the high-n data set.

\subsubsection{Changes in Beam Position}

Since the average $g$-2 phase depends on the average position of beam (see Fig. 4.58, for example), slow changes in the average position of the beam during a fill would result in an early-to-late change in the phase. Such slow changes might be possible if, for example, the high voltage on the electrostatic quadrupoles were to droop over the $700 \mu$ s over which the data are fit. A droop in the quadrupole high voltage would also change the vertical width of the beam. The effects that changes in average vertical position and width of the beam have on the average $g-2$ phase were studied extensively by Q. Peng, R. Carey and J. Miller using G2GEANT [100], and limits on the changes in the vertical position of the beam 
were determined from FSD studies by D. Hertzog and C. Polly. The conclusion from these studies is that the beam position change systematic uncertainty is less than $0.02 \mathrm{ppm}$.

\subsubsection{Other Systematic Uncertainties}

The systematic uncertainties previously discussed are typically considered the more "dangerous" of all systematic effects, since they all cause the average $g$-2 phase to change with time. However, there are several other less dangerous systematic effects that must be studied. Here we discuss uncertainties arising from fill selection, "other betatron oscillations" (eg, vertical oscillations and double radial CBO), choice of bin width, fast rotation randomization, shifts in the pick-off time from the WFD, fit procedure and AGS background. Some of these effects are completely negligible, others result in uncertainties in $R$ of comparable size to those determined for the more dangerous systematic effects.

\section{Fill Selection}

As discussed in Section 4.1.2, various cuts were made on each fill in order to filter out such potential problems as laser contamination, quad sparking, misreading the T0 pulse, and narrow pulse contamination. If we could assume that these cuts are $100 \%$ efficient, we would not need to assign a corresponding systematic error on the precession frequency. However, when the data rate flowing through the DAQ is very high, the data may be misread or fills may become misaligned between the various VME crates. Therefore, there is the possibility of using some data that are corrupted in some way.

During the run selection process, C. Polly looked for coincidences between various noncontiguous detectors in order to eliminate runs that may have a large amount of laser contamination. If after applying a cut on the laser flag one finds many coincidences between detectors, then it is very likely that the laser flag was misread. Since no laser runs were found that showed a large number of coincidences when the laser flag was not set, we may assume the laser cut to be $100 \%$ efficient.

We may estimate the level of contamination of DAQ misreadings in the other non-laser runs from Polly's work. No DAQ misreadings were found for approximately $1.2 \times 10^{7}$ pulses. Since there are approximately 80 pulses on average per fill, this means Polly looked at approximately 150,000 fills. We therefore set an upper limit on the fraction of DAQ misreadings in the data set at approximately $7 \mathrm{ppm}$.

To set an upper limit on the systematic errors on $R$ due to quadrupole cut inefficiencies, five parameter decay electron time spectra were generated where a fraction of the decay electron times were truncated at $225 \mu \mathrm{s}$ after injection. This would happen if, for example, the fill cuts failed to catch a quadrupole spark. The fraction of truncated data was set to both 10 and $50 \mathrm{ppm}$. Fitting the data to the 3-parameter ratio function we find the largest systematic shift in $R$ to be less than $0.00005 \mathrm{ppm}$, which we neglect.

The $T 0$ cut applied to each fill requires that the signal from the $T 0$ counter has a nonzero value between 50-65 $\mu$ s and an amplitude in the WFD greater than 30 ADC counts. The relationship between these $T 0$ requirements was investigated, and a $100 \%$ correlation was found between them. Therefore the only way that the $T 0$ signal could be wrong is if, because of a DAQ failure, the wrong T0 time is used. However, the distribution of T0 per fill is fairly narrow, with a width of a few nanoseconds. Using a "wrong" T0 would 
therefore mean using a $T 0$ that is shifted by a nanosecond or two, which would only dilute the $g$-2 asymmetry in the data (thereby increasing the statistical error), and would cause no early-to-late shift in the observed energies or times of decay electron pulses. We therefore may safely ignore this issue.

Finally, we compare the fit results from before and after the narrow pulse cut was applied. The final results changed by $0.2 \mathrm{ppm}$ for the low n-value data, and less than $0.05 \mathrm{ppm}$ for the high n-value data. The narrow pulse cut is based on Q. Peng's narrow pulse studies, and he finds[101] that of the 19,617 and 4,652 two-peak narrow pulses above $1.8 \mathrm{GeV}$ that he finds in the low-n and high-n data respectively, 268 and 128 two-peak narrow pulses remain after applying a cut on the average fit $\chi^{2}$ per fill. Therefore the cut is approximately $1.4 \%$ and $2.8 \%$ inefficient for the low-n and high-n data sets respectively. This implies a systematic error on $R$ of $0.003 \mathrm{ppm}$ and $0.001 \mathrm{ppm}$ for low-n and high-n data sets respectively.

\section{Other Betatron Oscillations}

Besides radial $\mathrm{CBO}$, the decay electron counting rate is also modified by other betatron oscillations. The three other dominant oscillations are the radial width of the beam, the vertical width of the beam, and the vertical betatron oscillation. The radial width oscillates at the frequency of twice the radial CBO frequency, and is therefore dubbed double CBO, or DCBO. The vertical betatron oscillation (VBO) frequency is

$$
f_{\mathrm{vbo}}=f_{c}(1-\sqrt{n})
$$

where $f_{c}$ is the cyclotron frequency, and vertical width, also referred to as the vertical waist $(\mathrm{VW})$, oscillates at the frequency

$$
f_{\mathrm{vw}}=f_{c}(1-2 \sqrt{n})
$$

Since the vertical oscillations are of rather high frequency, they dephase rapidly and are only seen in the data at very early times after injection. All oscillation frequencies are seen in the early-time residuals of fits to the data at late times. Furthermore, the radial width oscillations of the beam are seen in the fiber harps, and the vertical oscillations are seen by the FSDs when one takes the difference of the time spectra of FSD tiles 2 and 4 (see Fig. 4.61-4.62). It should be noted that the vertical breathing of the beam was not seen by the FSDs when looking at other combinations of FSD tiles (eg: 3-2-4), however the frequency appears as a clear peak in the FFT of the early-time residuals of the fits to the data.

The effect of DCBO was added to the fit function by modifying the nine parameter ratio function such that

$$
\begin{aligned}
f_{0}(t)= & {\left[1+A_{N_{\mathrm{CBO}}}(t)+A_{N_{\mathrm{DCBO}}}(t)\right] \times\left[1+A\left(1+A_{A_{\mathrm{CBO}}}(t)\right) \times\right.} \\
& \left.\cos \left(\omega_{a} t+\phi_{a}\left(1+A_{\phi_{\mathrm{CBO}}}(t)\right)\right)\right]
\end{aligned}
$$

where

$$
A_{N_{\mathrm{DCBO}}}(t)=A_{N_{\mathrm{DCBO}}} e^{-2 t / \tau_{\mathrm{CBO}}} \cos \left(2 \omega_{\mathrm{CBO}} t+\phi_{N_{\mathrm{DCBO}}}\right)
$$



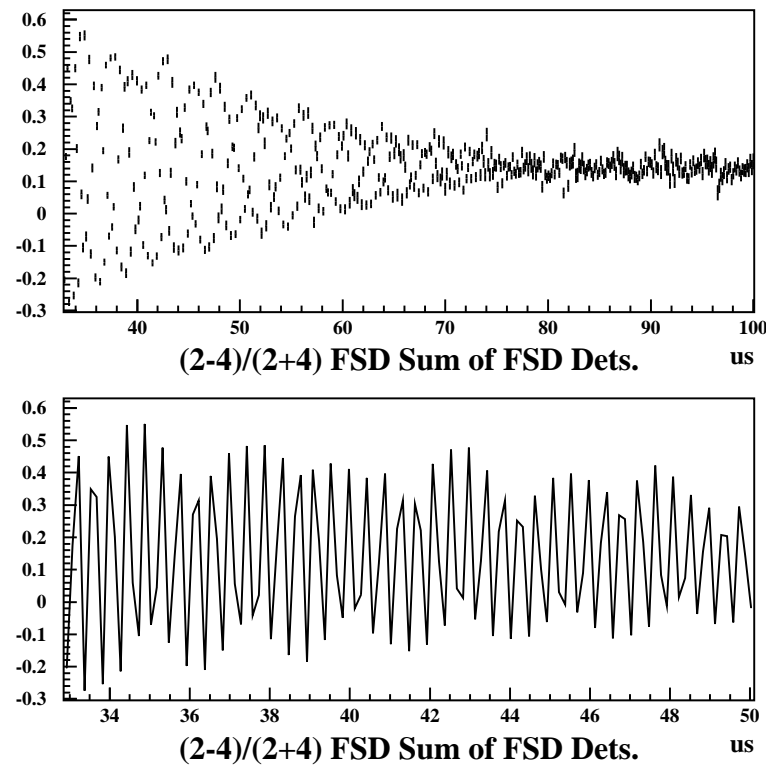

Figure 4.61: Vertical oscillations as seen by the FSDs by looking at the differences between tiles 2 and 4 as a function of time. Top: Sum of all "tile 2 - tile 4" time spectra from 33-100 $\mu \mathrm{s}$. Bottom: Same as top, but from 33-50 $\mu$ s, where the VBO oscillations are more easily seen.

$f_{ \pm}(t)$ were modified in a similar fashion. Note that the lifetime of DCBO is fixed to half the lifetime of the radial CBO, and the frequency is fixed to twice the radial CBO frequency. The plots in Fig. 4.63(a) show the distributions of differences in $R$ between fitting for DCBO and not fitting DCBO. The mean systematic error for both data sets is $\sim 0.025 \mathrm{ppm}$.

Vertical oscillations were similarly treated by modifying Eq. 4.38 to

$$
\begin{aligned}
f_{0}(t)= & {\left[1+A_{N_{\mathrm{CBO}}}(t)+A_{N_{\mathrm{vbo}}}(t)\right] \times\left[1+A\left(1+A_{A_{\mathrm{CBO}}}(t)\right) \times\right.} \\
& \left.\cos \left(\omega_{a} t+\phi_{a}\left(1+A_{\phi_{\mathrm{CBO}}}(t)\right)\right)\right]
\end{aligned}
$$

where

$$
A_{N_{\mathrm{vbo}}}(t)=A_{N_{\mathrm{vbo}}} e^{-t / \tau_{\mathrm{vbo}}} \cos \left(\omega_{\mathrm{vbo}} t+\phi_{N_{\mathrm{vbo}}}\right)
$$

$f_{ \pm}(t)$ were modified similarly. The VBO lifetime is fixed to $28 \mu \mathrm{s}$, obtained from fits to the FSD tile 2-tile 4 spectra, and the VBO frequency is calculated given the assumed CBO frequency in the fit:

$$
f_{\mathrm{vbo}}=f_{c} \times \sqrt{1-\left(1-\left(f_{\mathrm{CBO}} / f_{c}\right)\right)^{2}}
$$

where $f_{c}=6.703$ is the cyclotron frequency in $\mathrm{MHz}$ and $f_{\mathrm{CBO}}$ is the assumed CBO frequency. The plots in Fig. 4.63(b) show the $d R$ distributions for both data sets between fitting for VBO and not fitting VBO. The mean systematic error for the low-n data set is less than $0.01 \mathrm{ppm}$ and $\sim 0.02 \mathrm{ppm}$ for the high-n data set.

Finally, the vertical waist was treated the same as vertical oscillations, with $A_{N_{\mathrm{vbo}}}(t)$ replaced by $A_{N_{\mathrm{vw}}}(t), f_{\mathrm{vbo}}$ replaced with $f_{\mathrm{vw}}$ (calculated from the assumed CBO frequency 

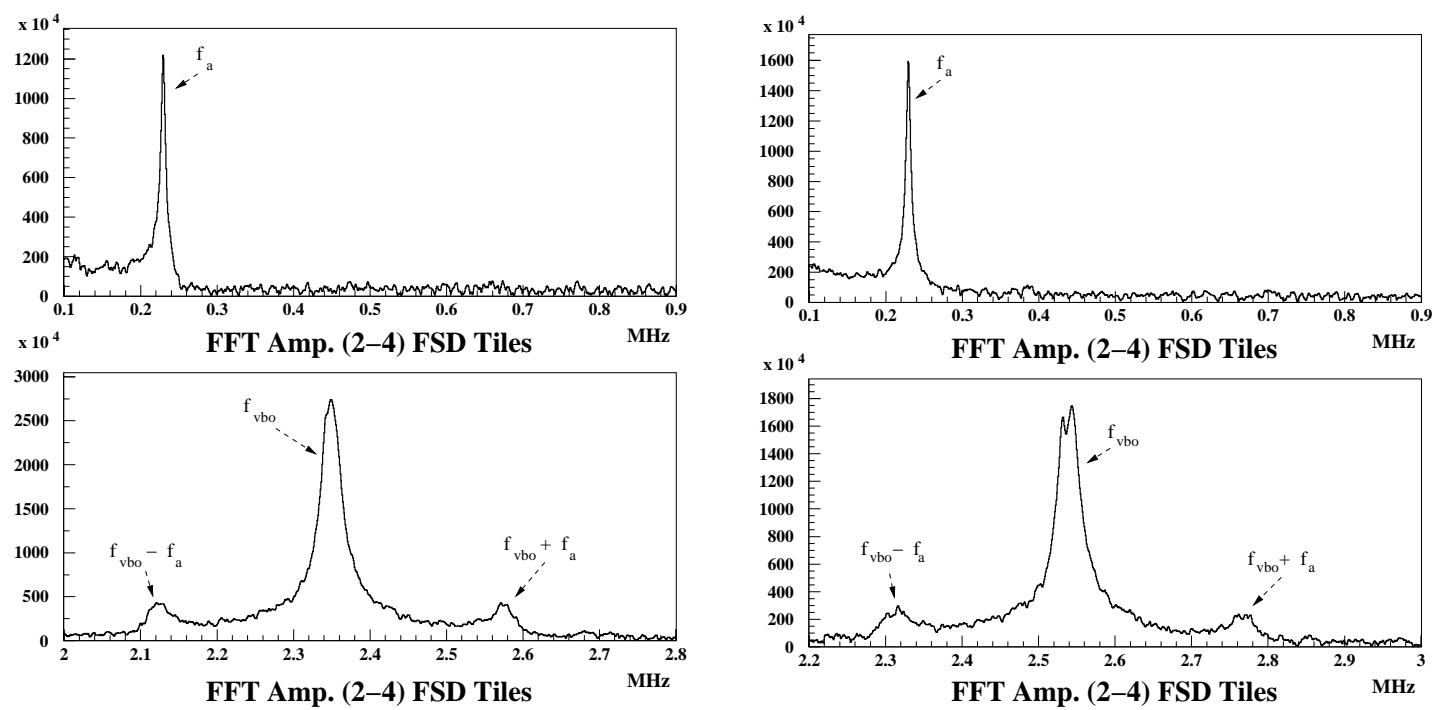

(a) Low n-value data.

(b) High n-value data.

Figure 4.62: FFT of FSD "tile 2 - tile 4" time spectra. Top: low frequency range, showing a $g$-2 peak. Bottom: high frequency range, showing the vertical BO peak.

in the fit) and $\tau_{\mathrm{vw}}$ is set to $20 \mu \mathrm{s}$. The VW lifetime was obtained by fitting the relative FFT amplitudes of the VW peak as a function of time, where the FFT spectra were binned in separate $g$-2 periods (that is, FFT of residuals from 20-24.365, 24.365-28.73, etc.) The plots in Fig. 4.63(c) show the $d R$ distributions for both data sets between fitting for VW and not fitting for it. The mean systematic error in ignoring VW in the fits is $\sim 0.02 \mathrm{ppm}$ for both data sets. Adding all error in quadrature, we find the total other betatron oscillation systematic error on $R$ for the low-n data set to be $0.03 \mathrm{ppm}$, and $0.04 \mathrm{ppm}$ for the high-n data set.

\section{Bin Width and Randomization}

The bin width systematic uncertainty has been studied in past years [102], and since there is no new physics involved in this year's analysis, we use the previous study's results: $d R_{\mathrm{bw}}=0.05 \mathrm{ppm}$. As for the elimination of the fast rotation structure in the data via fill randomization, the uncertainty on $R$ using only one random seed for fill randomization is $\sim 10 \%$ of the statistical uncertainty on $R$. Five different time spectra, each with different random seeds, were constructed for the final analysis. Using the RMS spread of the five fitted $R$-values to estimate the randomization systematic uncertainty, $\operatorname{RMS}(R) / \sqrt{5-1}$, we find $d R_{\text {rand }}=0.03 \mathrm{ppm}$ for the low-n data set and $0.09 \mathrm{ppm}$ for the high-n data set. 

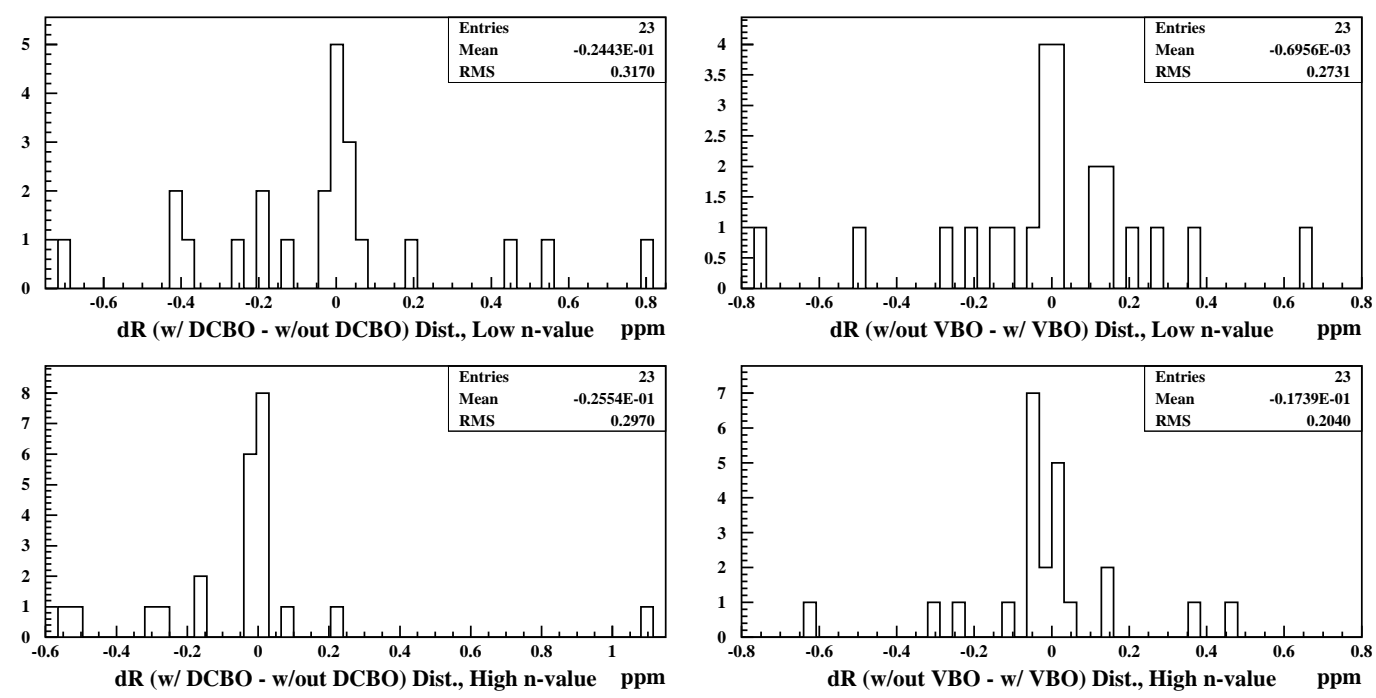

(a) Double CBO.

(b) Vertical BO.
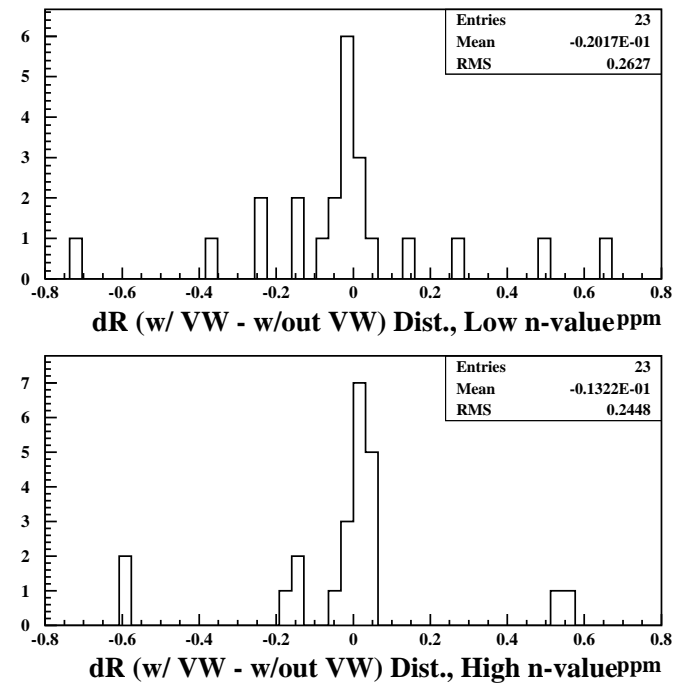

(c) Vertical Waist.

Figure 4.63: Effect on the fit values of $R$ when neglecting the various other betatron oscillation terms. $d R=R_{\text {obo }}-R_{9}$, where $R_{\text {obo }}$ is the fit value of $R$ with the "other betatron oscillation" term included, and $R_{9}$ is the nominal fit value of $R$ from the nine-parameter fit function. 

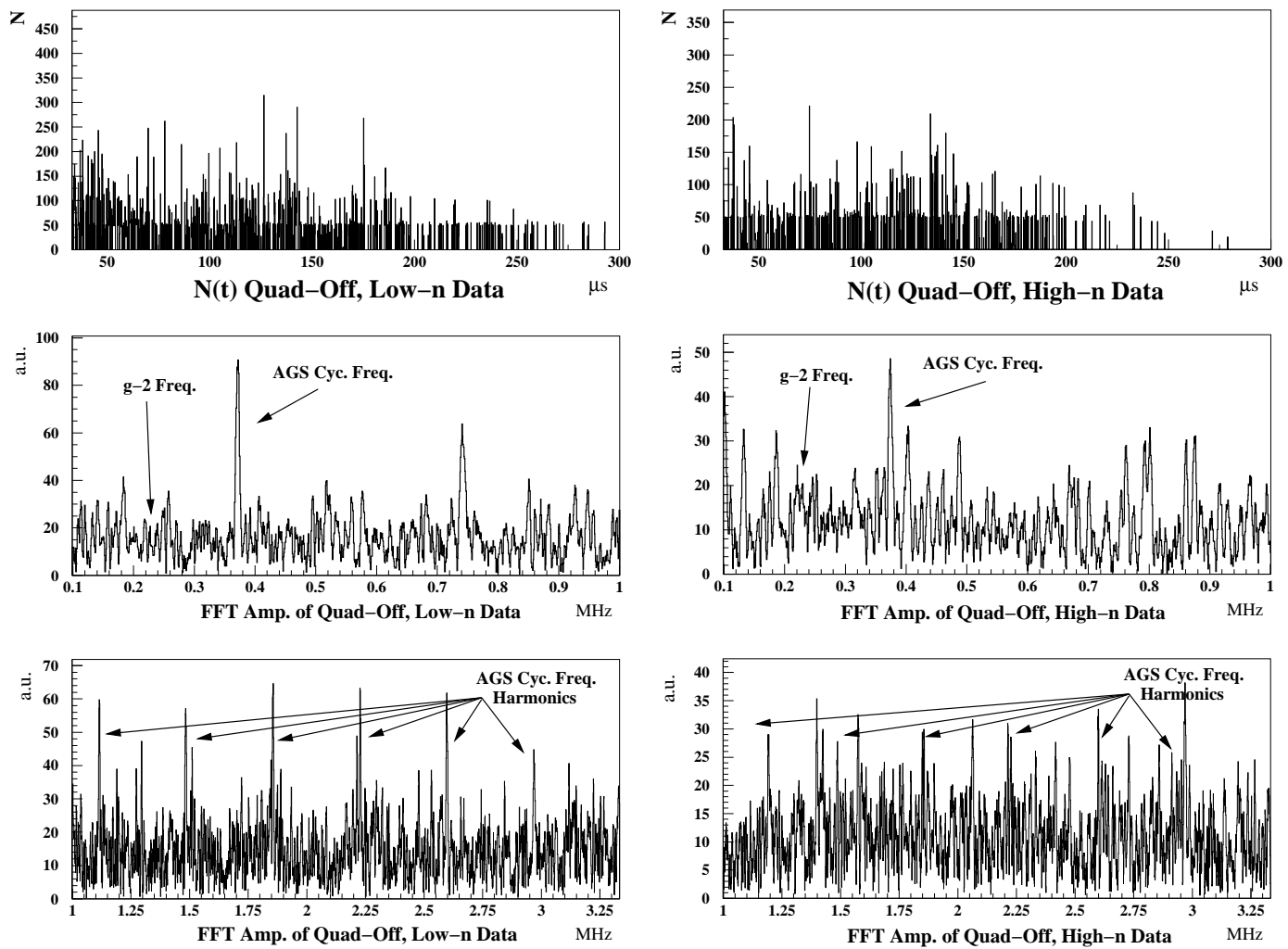

Figure 4.64: Flashlet time and frequency spectra, all detector data summed together for both data sets. The flashlets were measured by turning the quadrupoles off every $m^{\text {th }}$ fill. The $N(t)$ spectra are therefore scaled up by a factor of $m$, where $m$ is determined on a run-by-run basis (typically $m \simeq 49$ ).

\section{Timing Shifts}

The systematic error on $R$ from timing shifts in the pick-off time of pulses from the detectors has been determined by R. Carey to be $0.02 \mathrm{ppm}$ [103].

\section{AGS Background}

In the 2001 data run, the quadrupoles were turned off every " $m$ " fills, where $m$ was changed from 25 to 49 at some point near the beginning of the run. These "quad-off" fills allowed a measurement of the time and energy spectra of the AGS background (or flashlets). The top plots in Fig. 4.64 show the quad-off time spectra for both $n$-value data sets, and the bottom four plots show the FFT spectra in different frequency domains, $100-1000 \mathrm{kHz}$ for the middle plots and 1-3.3 MHz for the bottom plots. The AGS cyclotron frequency of $\sim 370 \mathrm{kHz}$ and its harmonics are clearly distinguishable; note also that there is no peak above noise at the $g-2$ frequency. The time spectra were built for each run, and the usual energy cuts were applied. The time spectra were later scaled-up by a factor of $m$, where $m$ is determined for each run (typically $m \simeq 49$ ). Fig. 4.65 shows the energy spectrum of the 


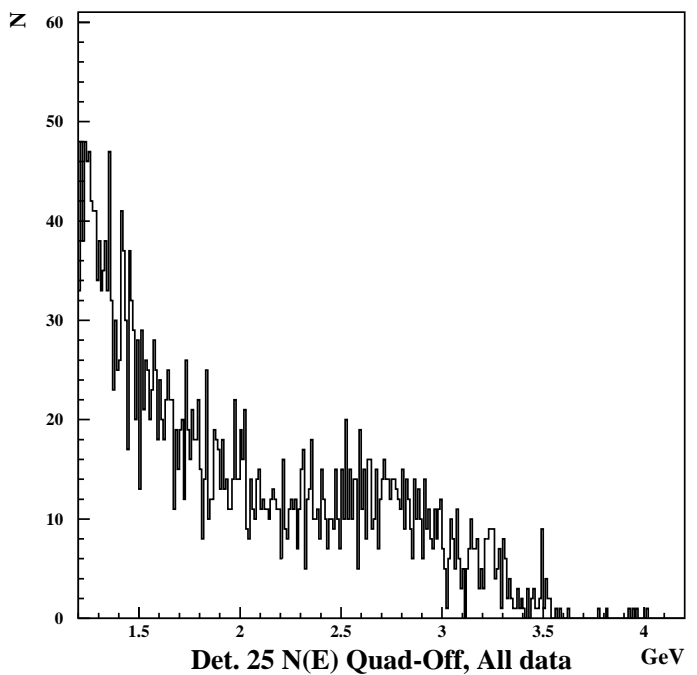

(a) Flashlet energy spectrum, from 1.2-4.2 $\mathrm{GeV}$.
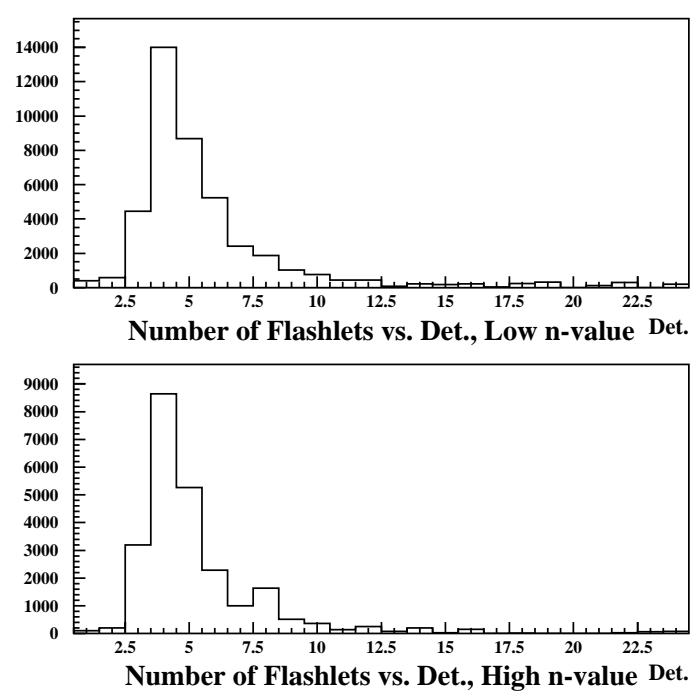

(b) Number of flashlets as a function of detector number.

Figure 4.65: Flashlet energy spectrum and distribution around the ring.

flashlets and the distribution of flashlets around the ring (number of flashlets vs. detector) for both data sets. We see that a significant fraction of flashlets deposit energies greater than our lower energy threshold in the calorimeters, and that most flashlets are observed in detectors just downstream of the injection point.

To determine the effect of AGS background on the fit value of $R$, the quad-off time spectra were added to the quad-on time spectra, with multiplicative factors of $-1.5,-1,-0.5$, $0,0.5,1$, and 1.5. Therefore, the multiplicative factor of -1 essentially subtracted out the AGS background from the data, and the factor of 1 doubled the amount of AGS background in the data. These time spectra were then fit to the 9-parameter ratio function; the largest difference between fit $R$ values from any of the fits was less than $0.01 \mathrm{ppm}$. We therefore claim an AGS background systematic uncertainty of $0.01 \mathrm{ppm}$.

\section{Fit Procedure}

In this analysis, limits were set on all fit parameters in order to ensure fit parameter stability and $\chi^{2}$ minimization convergence. However this procedure can be problematic with MINUIT, since if a parameter is found to be at or near its limit then MINUIT cannot properly evaluate the error on the parameter. Fairly loose limits were set on the important parameters, ie: $A$ and $R$, and $\phi_{a}$ had limits from 0 to $2 \pi$. The CBO amplitudes all had limits from 0 to 10 times the "typical" amplitudes found from the fits, and the CBO phases were also limited

to fall within 0 to $2 \pi$. Occasionally in the fit start time scans some of the CBO parameters were found to be at or very close to their limits. However, $R$ was never found anywhere near its limits. 


\begin{tabular}{|c||c||c|c||c|}
\hline \multicolumn{5}{|c|}{ Comparison of Radial Distributions } \\
\hline \hline Method & FFT & CERN & FFT & CERN \\
\hline Data Set & Low-n & Low-n & High-n & High-n \\
\hline$\langle x\rangle(\mathrm{cm})$ & 0.245 & 0.252 & 0.202 & 0.229 \\
\hline$\sigma_{x}(\mathrm{~cm})$ & 1.042 & 0.999 & 0.997 & 0.991 \\
\hline$\left\langle x_{e}^{2}\right\rangle$ & 1.146 & 1.062 & 1.035 & 1.035 \\
\hline$C_{E_{r}}(\mathrm{ppm})$ & 0.49 & 0.45 & 0.50 & 0.50 \\
\hline
\end{tabular}

Table 4.6: Comparison of the means and widths of the radial distributions obtained by the two fast rotation analyses, and the radial electric field correction obtained using their results.

Still, to be sure that the value and errors of $R$ were not systematically pulled, a study was done where the limits on $R$ were removed. About $90 \%$ of the fits converged, and the average difference between those $R$ values found with limits and those found without was $\sim-0.04 \pm 0.07 \mathrm{ppm}$. An order of magnitude smaller average difference (and also consistent with zero) was found between the errors on $R$. Similar studies were done where not only the limits on $R$ were removed, but also the limits on the CBO amplitudes were removed, and similar average differences $(\sim 0.04 \mathrm{ppm})$ were found. We therefore set the systematic error on $R$ due to fit procedure at $0.04 \mathrm{ppm}$.

\section{Radial Electric Field and Vertical Pitch Corrections}

As discussed in Section 2.4.2, the radial electric field correction is

$$
C_{E_{r}}=2 n(1-n)\left(\frac{\left\langle x_{e}^{2}\right\rangle}{\rho^{2}}\right)
$$

where, if one knows the radial distribution,

$$
\left\langle x_{e}^{2}\right\rangle=\langle x\rangle^{2}+\sigma_{x}^{2}
$$

$x$ is with respect to the central orbit, that is, $711.2 \mathrm{~cm}$. Table 4.4 .7 summarizes the electric field corrections for each fast-rotation analysis and data set, where Eq. 4.68 is used assuming $n=0.122$ and $n=0.142$ for the low- and high-n data sets respectively. Averaging the results from the two fast-rotation analyses, we find $C_{E_{r}}($ low-n $)=0.47$ and $C_{E_{r}}$ (high-n) $=0.50 \mathrm{ppm}$.

Unfortunately since no systematic error analysis has been made for either of the 2001 fast-rotation analyses, we do not know what the systematic uncertainties are on $\langle x\rangle$ and $\sigma_{x}$. One of the main issues with the radial distributions obtained from the fast rotation analyses are the tails of the distribution which exist even beyond the limits of the storage aperture. The radial distribution obtained from g2track has no such tails and the width is consistently smaller by about $0.05 \mathrm{~cm}$, or $5 \%$. Onderwater has pointed out [104] that the radial electric field correction changes by $10 \%$ when these tails are removed, which is consistent with a $5 \%$ error on the width. Therefore, due to the unphysical presence of the tails and their effect on the width, we claim a $5 \%$ uncertainty on the width, which translates to a $10 \%$ uncertainty on the correction. Tracking studies[105] have shown that 
uncertainties on the average vertical position of the beam result in an uncertainty of 0.02 $\mathrm{ppm}$ on the radial electric field correction. Adding the uncertainties of the width of the radial distribution ( $10 \%$ of $C_{E_{r}}$, or $\left.0.05 \mathrm{ppm}\right)$, and of the vertical position of the beam $(0.02$ $\mathrm{ppm}$ ) in quadrature, we find a total systematic uncertainty in $C_{E_{r}}$ of $0.054 \mathrm{ppm}$.

The vertical pitch correction is

$$
C_{P}=\frac{1}{2} \overline{\psi_{y}^{2}}=\frac{1}{2} n \frac{\left\langle y^{2}\right\rangle}{\rho^{2}}
$$

Unfortunately we do not have data from 2001 with which we may determine $\overline{\psi_{y}^{2}}$ or $\left\langle y^{2}\right\rangle$. We must therefore rely on traceback data from 1999 and tracking simulations. A distribution of the vertical width is obtained from traceback data collected in 1999: $\left\langle y^{2}\right\rangle=1.92 \mathrm{~cm}^{2}$. Once again, the uncertainty on this number is unknown. The $n$-value in this case was $\sim 0.135$. Under similar experimental settings, g2track found $\left\langle y^{2}\right\rangle=2.21 \mathrm{~cm}^{2}$. According to R. Carey [106], an acceptance correction of $-3 \%$ should be applied to this result, which gives $\left\langle y^{2}\right\rangle \simeq 2.14 \mathrm{~cm}^{2}$. The difference in $C_{P}$ obtained from these two numbers is $0.03 \mathrm{ppm}$, which we claim as the uncertainty. Tracking studies[105] have shown that uncertainties on the radial and vertical positions of the beam result in an uncertainty of $0.02 \mathrm{ppm}$ on $C_{P}$. Using the same $n$-values as were used during the 2001 data run, the values of $C_{P}$ determined from g2track are $C_{P}\left(\right.$ low-n) $=0.27 \mathrm{ppm}$ and $C_{P}$ (high-n) $=0.32 \mathrm{ppm}$.

Adding the two corrections together, and adding the systematic uncertainties together in quadrature, the total corrections $\left(C_{t}=C_{E_{r}}+C_{P}\right)$ to be applied to the final values of $\omega_{a}$ are $C_{t}$ (low-n) $=0.74 \pm 0.065 \mathrm{ppm}$, and $C_{t}$ (high-n) $=0.82 \pm 0.065 \mathrm{ppm}$.

\subsection{Summary}

Table 4.7 summarizes the average values of the $\chi^{2} /$ d.o.f. of the fits to $R$ vs. Detector, the resulting average $R$-values, and the asymmetry, phase and CBO amplitudes determined from the nine-parameter ratio fits. The final $R$ values determined from both data sets are very consistent with each other: $R_{\text {low }}=108.21 \pm 0.91 \mathrm{ppm}$ and $R_{\text {high }}=108.42 \pm 1.10$ ppm. The results for the smaller Silver+Bronze set of data are also in excellent agreement: $R_{S B}=110.55 \pm 3.73$. The uncertainties stated here are statistical.

The systematic uncertainties for all three data sets are summarized in Table 4.8. The systematic errors for the "S+B" data set are for the most part assumed to be the same as for the "golden" low-n subset with a few exceptions. Since there was less scraping and a lower muon kick for these runs, we expect a larger fraction of muon losses. Therefore, the muon loss systematic error is conservatively estimated to be twice the size for the $\mathrm{S}+\mathrm{B}$ data set than for the golden low-n data set. Also the randomization systematic uncertainty for this data set is obtained independently in the same manner as for the other two data sets. In calculating the total systematic error, we assume the individual systematics errors are uncorrelated, and therefore all errors are added in quadrature to obtain a total systematic uncertainty of $0.21 \mathrm{ppm}$ for all data sets combined.

The final value of $\omega_{a}$ is obtained from a simple arithmetic mean of the results from the five different $\omega_{a}$ analyses. The analyses differ primarily in the applied energy cuts and fit functions used to extract $\omega_{a}$. Fig. 4.66 shows a comparison of the five results. The solid 


\begin{tabular}{|c||c||c||c|}
\hline \multicolumn{4}{|c|}{ Values of Fit Parameters } \\
\hline \hline $\begin{array}{c}\text { Fit } \\
\text { Parameter }\end{array}$ & $\begin{array}{c}\text { Low-n } \\
\text { Data Set }\end{array}$ & $\begin{array}{c}\text { High-n } \\
\text { Data Set }\end{array}$ & $\begin{array}{c}\text { S+B } \\
\text { Data Set }\end{array}$ \\
\hline $\begin{array}{c}\chi^{2} / \text { d.o.f. } \\
\text { of fit to } R \text { vs. Det. }\end{array}$ & $22.49 / 22$ & $36.72 / 22$ & $24.50 / 22$ \\
\hline$R \times 10^{-6}$ & $108.21(91)$ & $108.42(1.10)$ & $110.55(3.73)$ \\
\hline$A$ & $0.38138(3)$ & $0.38119(4)$ & $0.3816(1)$ \\
\hline$\phi_{a}$ & $3.0322(1)$ & $3.0323(2)$ & $3.0288(6)$ \\
\hline$A_{N_{c}} \times 10^{-2}$ & $0.922(10)$ & $1.28(14)$ & $1.99(36)$ \\
\hline$A_{A_{c}} \times 10^{-3}$ & $2.50(25)$ & $3.05(25)$ & $6.50(94)$ \\
\hline$A_{\phi_{c}} \times 10^{-3}$ & $6.62(83)$ & $5.80(77)$ & $2.08(30)$ \\
\hline
\end{tabular}

Table 4.7: Values of the fit parameters for the nine-parameter ratio fit. Results are for one random seed, however $\chi^{2}$ 's of the fit to $R$ vs. Detector and the resulting $R$-values are averaged over five spectra built from different random seeds.

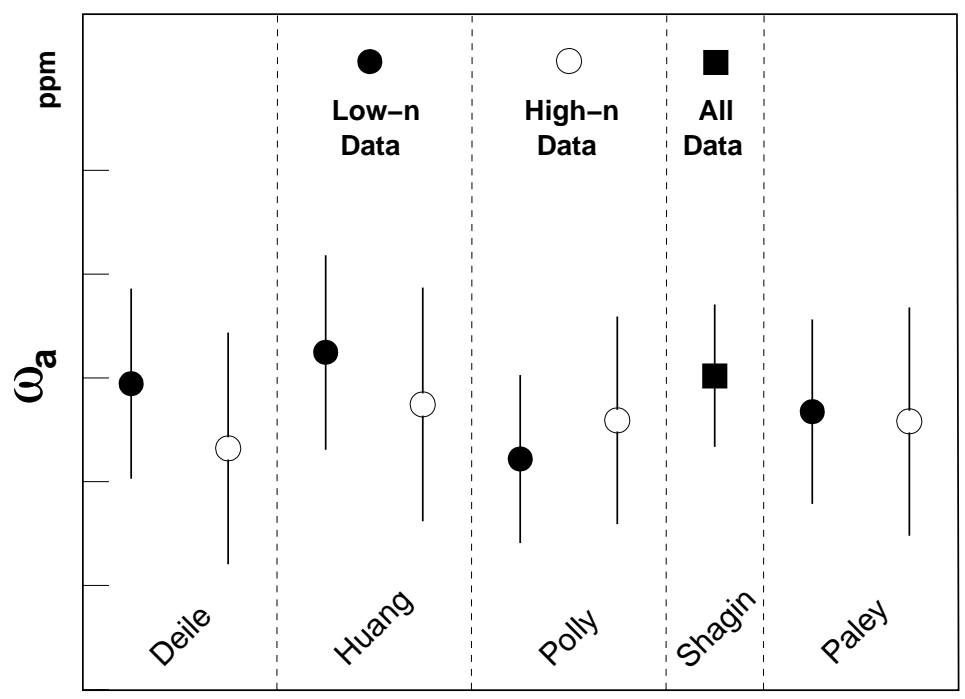

Figure 4.66: Comparison of the five $\omega_{a}$ analyses.

circles represent results from the low-n data set, the open circles represent results from the high-n data set, and the solid square represents the result of fits to all data combined. We note that in this plot the results for the low-n and $\mathrm{S}+\mathrm{B}$ data sets in this analysis were combined to a single low-n result.

The first three analyses extracted $\omega_{a}$ from fits to the decay electron time spectra. Deile and Huang both fit the decay electron time spectra to functions with parameterizations for $\mathrm{CBO}$ effects and muon losses, with slight differences in their implementation. Both used electron energies in the range of 1.8 to $3.4 \mathrm{GeV}$, and fit the combined detector data starting at $\sim 32 \mu$ s after injection. Polly also fit the decay electron time spectra to a parameterized function, however the counts were weighted with the energy-dependent asymmetry. This 


\begin{tabular}{|c||c||c||c||c|}
\hline \multicolumn{4}{|c|}{ Systematic Error Table (all units in ppm) } \\
\hline \hline $\begin{array}{c}\text { Syst. } \\
\text { Error }\end{array}$ & $\begin{array}{c}\text { Low-n } \\
(\mathrm{L})\end{array}$ & $\begin{array}{c}\text { High-n } \\
(\mathrm{H})\end{array}$ & $\begin{array}{c}\text { Silver+Bronze } \\
(\mathrm{S}+\mathrm{B})\end{array}$ & $\begin{array}{c}\text { Total } \\
(\mathrm{L}+\mathrm{H}+\mathrm{S}+\mathrm{B})\end{array}$ \\
\hline ESC & 0.01 & 0.02 & 0.01 & 0.01 \\
\hline WW & 0.1 & 0.1 & 0.1 & 0.1 \\
\hline Pileup & 0.06 & 0.05 & 0.06 & 0.05 \\
\hline CBO & 0.06 & 0.12 & 0.06 & 0.08 \\
\hline $\begin{array}{c}\text { Muon } \\
\text { Losses }\end{array}$ & 0.11 & 0.09 & 0.21 & 0.10 \\
\hline $\begin{array}{c}\text { Random- } \\
\text { ization }\end{array}$ & 0.04 & 0.05 & 0.18 & 0.05 \\
\hline $\begin{array}{c}\text { Timing } \\
\text { Shifts }\end{array}$ & 0.02 & 0.02 & 0.02 & 0.02 \\
\hline $\begin{array}{c}\text { Bin } \\
\text { Width }\end{array}$ & 0.05 & 0.5 & 0.05 & 0.05 \\
\hline Other BO & 0.03 & 0.04 & 0.03 & 0.03 \\
\hline AGS Bgnd & 0.01 & 0.01 & 0.01 & 0.01 \\
\hline $\begin{array}{c}\text { Fit } \\
\text { Procedure }\end{array}$ & 0.04 & 0.04 & 0.04 & 0.04 \\
\hline Fill Cuts & 0.01 & 0.01 & 0.01 & 0.01 \\
\hline$C_{E_{r}} \& C_{P}$ & 0.06 & 0.06 & 0.06 & 0.06 \\
\hline $\begin{array}{c}\text { Beam } \\
\text { Pos. }\end{array}$ & 0.05 & 0.05 & 0.05 & 0.05 \\
\hline \hline $\begin{array}{c}\text { Total } \\
\text { Syst. } \\
\text { Error }\end{array}$ & 0.21 & 0.22 & 0.33 & \\
\hline $\begin{array}{c}\text { Stat. } \\
\text { Error }\end{array}$ & 0.91 & 1.10 & 3.73 & 0.21 \\
\hline
\end{tabular}

Table 4.8: Summary of systematic errors for the various individual and combined data sets. All units are in ppm.

method minimizes the statistical uncertainty of the fitted value of $\omega_{a}$, and permits the energy range of decay electrons to be extended. Furthermore, by using a fit function that parameterized all statistically significant perturbations in the decay electron time spectrum, Polly was also able to obtain an acceptable fit $\chi^{2} /$ d.o.f. at an average fit start time of $25 \mu \mathrm{s}$ after injection for the sum of data from those detectors that were gated-on early. Using the asymmetry weighted method, electrons in the range of 1.5 to $3.4 \mathrm{GeV}$ and earlier fit start times, Polly obtained a $10 \%$ improvement in the statistical uncertainty of $\omega_{a}$.

The last two analyses, including the author's, extracted $\omega_{a}$ from fits to the ratio. Shagin used electrons in the range 1.8-3.4 GeV, although with slightly different ADC to GeV conversion factors from those used in this analysis. Furthermore, Shagin took advantage of the fact that the CBO oscillations in the two data sets are nearly $180^{\circ}$ out-of-phase, and added all data together. This significantly reduced the amplitude of the half-ring effect, 
and his CBO systematic uncertainty is estimated from extensive MC simulation studies.

The fit results from the analyses have been shown to be in agreement to within the expected deviations from the differences in data selection and methods of extracting $\omega_{a}$. Adding the value of $R_{\text {off }}=-7.80 \mathrm{ppm}$ to the average value of $R$, and adding the radial electric field and vertical pitch corrections to the value of $\omega_{a}$, we find $f_{a}=\omega_{a} / 2 \pi=$ 229073.59(15)(5) Hz. The first uncertainty is statistical and the second is systematic, and both are calculated taking into account the correlations among the five analyses. The correlations are dominated by the overlap of data used in each analysis. 


\section{Chapter 5}

\section{Conclusion}

\subsection{Determination of $a_{\mu^{-}}$}

After removing the different offsets used in the $2001 \omega_{p}$ and $\omega_{a}$ analyses, the value of $a_{\mu^{-}}$ was calculated according to the relation

$$
a_{\mu}=\frac{\mathcal{R}}{\lambda-\mathcal{R}}=11659214(8)(3) \times 10^{-10}
$$

where $\mathcal{R}_{\mu^{-}}=\omega_{a} / \omega_{p}=0.0037072083(26)$ and $\lambda=\mu_{\mu} / \mu_{p}=3.18334539(10)$ [44]. The first error is statistical and the second is systematic. The measured value of $\mathcal{R}_{\mu^{-}}$is in good agreement with the average of the previous measurements of $\mathcal{R}_{\mu^{+}}=0.0037072048(25)$, the difference being $\Delta \mathcal{R}=(3.5 \pm 3.4) \times 10^{-9}$. Assuming charge, parity and time $(\mathrm{CPT})$ symmetry and taking into account correlations between systematic uncertainties between the various data sets, the new average value of $\mathcal{R}_{\mu}=0.0037072063(20)$. From this result we obtain the new average value of $a_{\mu}^{\exp }=11659208(4)(3) \times 10^{-10}$, a relative uncertainty of $0.5 \mathrm{ppm}[107]$.

\subsection{Discussion}

Fig. 5.1 shows a comparison between the experimental and theoretical values of $a_{\mu}$. The differences are

$$
\Delta a_{\mu}= \begin{cases}(27.0 \pm 10.0) \times 10^{-10} & \left(e^{+} e^{-}-\text {based }\right) \\ (12.3 \pm 9.2) \times 10^{-10} & (\tau-\text { based })\end{cases}
$$

\subsubsection{Muon Electric Dipole Moment}

The electric dipole moment (EDM) is defined as

$$
\vec{d}=\frac{\eta}{2}\left(\frac{e}{4 m}\right) \vec{S}
$$

Lepton EDMs are expected to be extremely small in the Standard Model, $d_{e} \simeq-1.5 \times$ $10^{-38} e \cdot \mathrm{cm}$ and $d_{\mu} \simeq-3 \times 10^{-36} e \cdot \mathrm{cm}$ [108]. The current experimental limit on the electron 


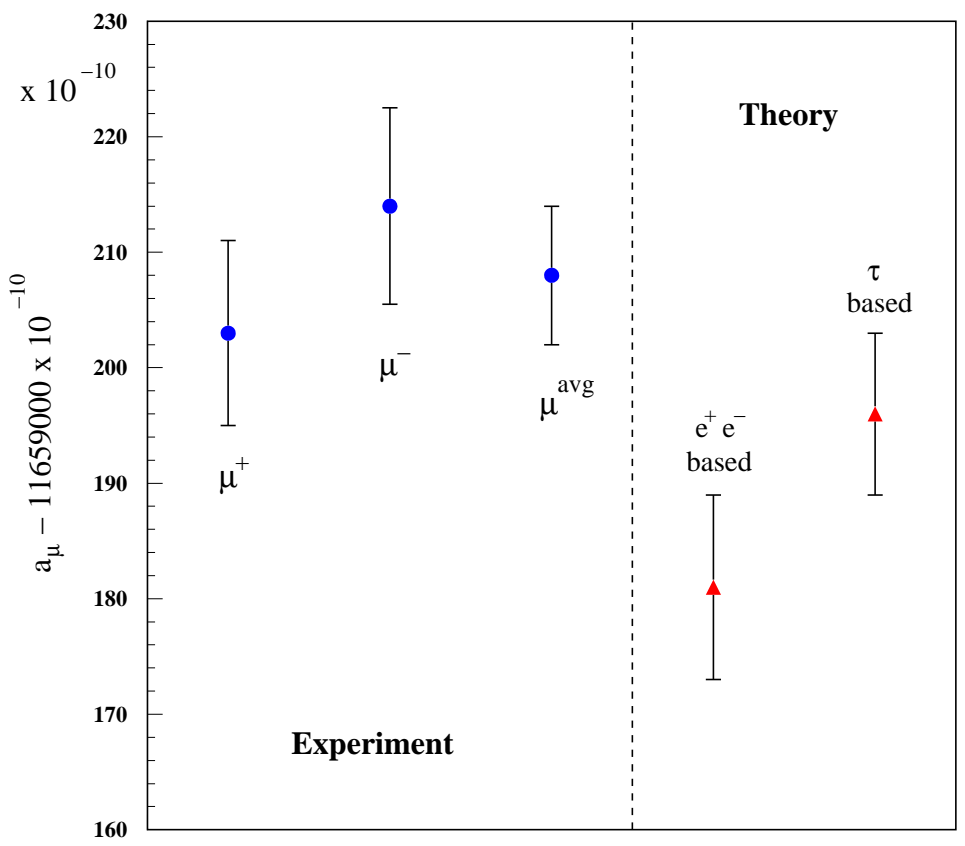

Figure 5.1: Comparison of the most current experimental and theoretical values for $a_{\mu}$.

EDM is $\left|d_{e}\right|<1.5 \times 10^{-27} e \cdot \mathrm{cm}(95 \% \mathrm{CL})$. From naive mass-scaling arguments $\left(d_{l} \sim m_{l} / m_{\Lambda}\right.$, so $\left.d_{\mu} \sim m_{\mu} d_{e} / m_{e}\right)$ one expects $\left|d_{\mu}\right|<3.1 \times 10^{-25} e \cdot \mathrm{cm}(95 \% \mathrm{CL})$. Unfortunately the experimental limit on the muon EDM is nearly six orders of magnitude larger. A limit on the muon EDM may be set using the difference between the experimental and theoretical values of $a_{\mu}$. However, $\left|d_{\mu}\right|$ may be obtained experimentally and independently of $a_{\mu}$ by measuring from an up-down asymmetry in the number of observed decay electrons. Both approaches use the fact that an EDM changes the equation for the spin difference frequency.

In the case of $\gamma=\gamma_{\text {magic }}$ and neglecting $\vec{\beta} \cdot \vec{B}$ and $\vec{\beta} \times \vec{E}$ terms, $\omega_{a}$ becomes

$$
\vec{\omega}_{a}=\frac{e}{m} a_{\mu} \vec{B}+2 d(\vec{E}+\vec{\beta} \times \vec{B})
$$

The $2 d \vec{E}$ term is negligible. Therefore the measured spin frequency depends on both $a_{\mu}$ and $d$ :

$$
a_{\mu}^{\exp }=\sqrt{\left(a_{\mu}^{\mathrm{SM}}\right)^{2}+\left(\frac{m}{e}\right)^{2}(2 d)^{2}}
$$

so that

$$
d=\frac{1}{2} \frac{e}{m} \sqrt{\left(a_{\mu}^{\exp }\right)^{2}-\left(a_{\mu}^{\mathrm{SM}}\right)^{2}}
$$

Assuming no measurable contributions to $a_{\mu}$ from physics beyond the standard model, and using the latest experimental value and theoretical estimate for $a_{\mu}$, we find that

$$
d= \begin{cases}(2.38 \pm 0.83) \times 10^{-19} e \cdot \mathrm{cm} & \left(e^{+} e^{-}-\text {based }\right) \\ (1.79 \pm 1.05) \times 10^{-19} e \cdot \mathrm{cm} & (\tau-\text { based })\end{cases}
$$


From Eq. 5.4 we see that the presence of an EDM gives the spin precession frequency vector a radial component (assuming $\left.\vec{B}=\left(0, B_{y}, 0\right)\right)$. Therefore the plane of the spin precession is tilted in the vertical direction by an angle

$$
\delta=\tan ^{-1}\left(\frac{\eta \beta}{2 a_{\mu}}\right)
$$

This tilt results in a time-dependent, up-down asymmetry in the decay rate. Limits on the muon EDM may be placed by searching for an asymmetry in the number of decay electrons between the upper and lower halves of our detectors. An analysis [108] of the 2000 FSD and calorimeter data from E821 places a new limit for the muon EDM: $\left|d_{\mu}\right|<2.7 \times 10^{-19} e \cdot \mathrm{cm}$ (95\% CL).

We note that, although the limits on the muon EDM are rather weak, it is unlikely that the difference $\Delta a_{\mu}$ arises from $d_{\mu}$. Although new physics, such as SUSY, produces a larger muon EDM than that of the SM prediction, these EDMs are still predicted to be at least 3-4 orders of magnitude smaller than the current limits (in fact, the electron EDM imposes quite stringent constraints on new physics models). Therefore, in the following discussion, we ignore the effect of an EDM.

\subsubsection{Limits on New Physics}

\section{SUSY Particle Masses}

As discussed in Section 1.5, $\Delta a_{\mu}$ plays a significant role in placing limits on supersymmetric particle masses. We see from Eq. 1.38,

$$
\Delta a_{\mu} \simeq 13.0 \times 10^{-10}\left(\frac{100 \mathrm{GeV}}{M}\right)^{2} \tan \beta= \begin{cases}27.0 \times 10^{-10} & \left(e^{+} e^{-}-\text {based }\right) \\ 12.3 \times 10^{-10} & (\tau \text {-based })\end{cases}
$$

that $M \simeq \mathcal{O}(100 \sqrt{\tan \beta} \mathrm{GeV})$. In fact, one may argue that if the supersymmetric particle mass spectrum is determined, $\Delta a_{\mu}$ places the strongest constraint on $\tan \beta$.

A more generic approach may be made by using other physics constraints. Since the lightest supersymmetric particle is a likely candidate for non-baryonic cold dark matter, astrophysical constraints, such as those imposed by the latest Wilkinson Microwave Anisotropy Probe (WMAP) data [109], may be used to place limits on the masses of the two lightest supersymmetric particles, $m_{0}$ and $m_{\frac{1}{2}}$. Fig. 5.2 shows a plot of the allowed regions of the $\left(m_{0}, m_{\frac{1}{2}}\right)$ plane for the constrained minimal supersymmetric standard model [110].

Fig. 5.2 assumes $\tan \beta=10$ and $\mu>0$ (where $\mu$ is a supersymmetric parameter similar to the Higgs boson mass in the Standard Model). Lines are drawn for the lower limits of the Higgs mass $\left(m_{h} \simeq 114 \mathrm{GeV}\right)$ and chargino mass $\left(m_{\chi^{ \pm}} \simeq 104 \mathrm{GeV}\right)$, set by direct searches at the Large Electron Positron (LEP) collider [110]. The darkly shaded regions are excluded by either direct searches for neutral dark matter (the region of low $m_{0}$ and high $m_{\frac{1}{2}}$ ) or by limits set by $b \rightarrow s \gamma$ [110] (the region of low $m_{0}$ and $m_{\frac{1}{2}}$ ). The preferred regions of the $\left(m_{0}, m_{\frac{1}{2}}\right)$ plane are the shaded regions labeled " $g$ - 2 " (from $\Delta a_{\mu}\left(e^{+} e^{-}\right.$-based)) and WMAP (the dark WMAP region is from the latest data, whereas the lighter region is from 


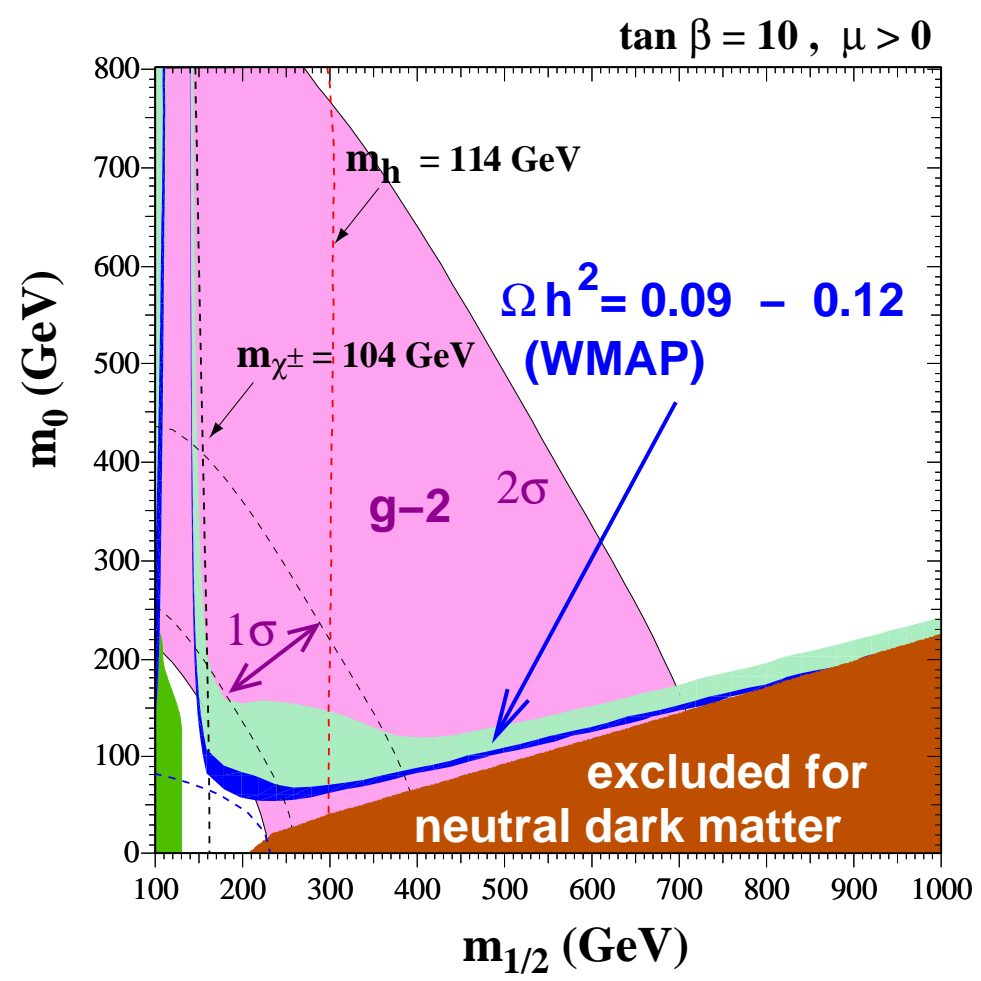

Figure 5.2: Allowed regions for masses of the two lightest supersymmetric partner particles. The calculation assumes a Higgs mass $\left(m_{h}\right)$ of $114 \mathrm{GeV}$, a chargino mass $\left(m_{\chi}\right)$ of $104 \mathrm{GeV}$, $\tan \beta=v_{1} / v_{2}=10, \mu>0$. The masses used here are from limits set by direct searches at the LEP collider. Darkly shaded regions are excluded, whereas the shaded regions marked as WMAP and $g-2\left(e^{+} e^{-}\right.$-based) constraints are preferred. Plot courtesy of K. Olive [111].

old data). The clearly demonstrates that, once the Higgs mass is determined, the combined constraints from $\Delta a_{\mu}$ and WMAP will determine the value of $\tan \beta$.

\section{Muon Substructure}

Although the muon is thought to be a point particle, this is only true at the level of experimental precision $\left(\sim 10^{-16} \mathrm{~cm}\right)$ If at the scale $\Lambda_{\mu} \gg m_{\mu}$ the muon is a composite fermion, a model-independent limit of the energy scale of the substructure may be estimated from its contribution to $a_{\mu}[112]$ :

$$
a_{\mu}\left(\Lambda_{\mu}\right) \simeq\left(\frac{m_{\mu}}{\Lambda_{\mu}}\right)^{2}
$$

Assuming the entire difference $\Delta a_{\mu}$ may be ascribed to muon substructure, we find

$$
\begin{array}{ll}
1.5 \mathrm{TeV}<\Lambda_{\mu}<4.0 \mathrm{TeV} & \left(e^{+} e^{-} \text {-based, 95\% CL }\right) \\
1.9 \mathrm{TeV}<\Lambda_{\mu}<\infty & (\tau \text {-based, 95\% CL })
\end{array}
$$




\section{Extra Dimensions}

The mathematical groundwork for extending the laws of physics to more than our usual four dimensions was set in the early part of the last century by Nordström, Kaluza and Kline. Recently the idea that the four dimensions we "live" in are confined to a "brane" (subspace) in a larger space of $4+n$ dimensions has become quite popular. For example, gravity could exist in $n$-compact dimensions, and if the fundamental scale for the gravitational interactions occurs at the TeV scale, then large compact extra dimensions could exist which would solve the hierarchy problem[113].

Although the precision electroweak measurements already place stringent constraints on the scale of such new physics, $a_{\mu}$ provides complementary constraints independent of the number of extra dimensions[114]. For example, Graesser [114] finds that in general the one-loop compact extra-dimensional contribution to the muon anomalous magnetic moment goes as

$$
a_{\mu}^{\mathrm{ED}}(1) \sim\left(m_{\mu} / \Lambda_{\mathrm{ED}}\right)^{2}
$$

"ED" stands for extra dimensions and $\Lambda_{\mathrm{ED}}$ is the scale at which the extra-dimensions become compactified. Therefore, although $\Lambda_{\mathrm{ED}}$ depends on the particular model of compactification, we see that with the latest value of $\Delta a_{\mu}, \Lambda_{\mathrm{ED}} \sim \mathcal{O}(1) \mathrm{TeV}$. This is similar to the limits set by the LEP collider data.

\subsection{Future Prospects}

Although the current discrepancy between the $e^{+} e^{-}$- and $\tau$-based calculations of $a_{\mu}$ makes interpretation of the experimental value difficult at best, progress is being made that should help to resolve this issue. In particular, the recent measurements of $\sigma\left(e^{+} e^{-} \rightarrow\right.$ hadrons) by KLOE using radiative return have confirmed the CMD-2 results [115]. This strengthens the case for the $e^{+} e^{-}$-based calculations. Another radiative return analysis is being done by the BaBar Collaboration that should result in a smaller systematic uncertainty than the KLOE result. Finally, more studies on the effect of the mass and width differences between $\rho^{+}$and $\rho^{0}$ on the $\tau$-based result are in progress.

Assuming the issues are resolved and systematic and statistical uncertainties of the first order hadronic vacuum polarization contribution are brought to a negligible level, the hadronic light-by-light remains a concern if the uncertainty in the calculation is to be reduced. Since no data-driven approach similar to that used for $a_{\mu}^{\mathrm{Had}}(1)$ exists, this contribution poses the biggest challenge in the theoretical evaluation of $a_{\mu}$. However, historically there has been progress in the calculation of $a_{\mu}^{\mathrm{SM}}$ as progress is made on $a_{\mu}^{\exp }$, and there is no reason to believe this trend would not continue if the uncertainty of $a_{\mu}^{\exp }$ were further reduced.

In this context, we remind the reader that this is the final result of experiment E821 at BNL. Because of the muon $g$-2's unique capacity for placing stringent limits on new physics, in particular SUSY models, we feel that further improvement on the measurement of $a_{\mu}$ is not only interesting but necessary. A factor of two improvement in the uncertainty of $a_{\mu}^{\exp }$ is possible with minor changes to the experimental setup at BNL[116]. A proposal has also been submitted to J-PARC for another muon $g$-2 experiment with a factor 10 improvement on the current experimental uncertainties[117]. Although much work is required to make 
such endeavors possible, it would be well worth the effort. Lepton magnetic moments have played a crucial role in the development of our understanding of nature for the last fifty years, and hopefully they will continue to do so for another fifty! 


\section{Appendix A}

\section{G2OFF Ntuple Structure}

\begin{tabular}{|c|c|c|c|c|c|}
\hline \multicolumn{6}{|c|}{ G2OFF Ntuple ID=10, General Data Block } \\
\hline Var numb & Type & Packing & Range & Block & Name \\
\hline 1 & $\mathrm{U}^{*} 4$ & 12 & {$[0,4095]$} & GENERAL & 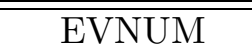 \\
\hline 2 & $\mathrm{U}^{*} 4$ & 5 & {$[1,16]$} & GENERAL & FILL \\
\hline 3 & $\mathrm{U}^{*} 4$ & 15 & {$[0,20000]$} & GENERAL & RUNNUM \\
\hline 4 & $\mathrm{U}^{*} 4$ & 16 & {$[0,60000]$} & GENERAL & IVERSION \\
\hline 5 & $I^{*} 4$ & 13 & {$[-2,4093]$} & GENERAL & IQUAD \\
\hline 6 & $\mathrm{R}^{*} 4$ & 16 & {$[0 ., 6553.5]$} & GENERAL & IONC \\
\hline 7 & $\mathrm{R}^{*} 4$ & 24 & {$[0 ., 1677721$.} & GENERAL & TLASER \\
\hline 8 & $\mathrm{U}^{*} 4$ & 1 & {$[0,1]$} & GENERAL & IOVER \\
\hline 9 & $\mathrm{R}^{*} 4$ & 16 & {$[0 ., 6553.5]$} & GENERAL & SECC \\
\hline 10 & $\mathrm{U}^{*} 4$ & 5 & {$[0,30]$} & GENERAL & DET \\
\hline 11 & $\mathrm{R}^{*} 4$ & 16 & {$[0 ., 6553.5]$} & GENERAL & TZERO \\
\hline 12 & $\mathrm{R}^{*} 4$ & & & GENERAL & TOffset \\
\hline 13 & $\mathrm{R}^{*} 4$ & & & GENERAL & RNUMB(10) \\
\hline 14 & $\mathrm{U}^{*} 4$ & & & GENERAL & HClOff \\
\hline 15 & $\mathrm{U}^{*} 4$ & & & GENERAL & HCLCK \\
\hline 16 & $\mathrm{R}^{*} 4$ & 16 & {$[-103.5,6450$} & GENERAL & QEarly(4) \\
\hline 17 & $\mathrm{R}^{*} 4$ & 16 & {$[-103.5,6450$} & GENERAL & $\operatorname{QMid}(4)$ \\
\hline 18 & $\mathrm{R}^{*} 4$ & 16 & {$[-103.5,6450$} & GENERAL & QLate(4) \\
\hline 19 & $\mathrm{R}^{*} 4$ & & & GENERAL & WFDStreak $(2)$ \\
\hline 20 & $\mathrm{R}^{*} 4$ & & & GENERAL & WFDLT(2) \\
\hline 21 & $\mathrm{R}^{*} 4$ & & & GENERAL & FDTime \\
\hline 22 & $I^{*} 4$ & & & GENERAL & MON90C \\
\hline 23 & $\mathrm{U}^{*} 4$ & & & GENERAL & MTDCFILL \\
\hline 24 & $\mathrm{U}^{*} 4$ & & & GENERAL & TOSTAT \\
\hline
\end{tabular}

Table A.1: GENERAL data block of the G2OFF decay electron ntuple data structure. This data block contains information regarding the run, detector, WFD quadrupole readout, common clock time between $\omega_{p}$ and $\omega_{a}$ DAQs, and other general data, for each AGS fill. 


\begin{tabular}{|c|c|c|c|c|c|}
\hline \multicolumn{6}{|c|}{ G2OFF Ntuple ID=10, WFD/Calo Pulse Data Blocks } \\
\hline Var numb & Type & Packing & Range & Block & Name \\
\hline 1 & 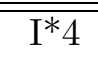 & & {$[0,500]$} & PULSES & NPULSE \\
\hline 2 & $\mathrm{R}^{*} 4$ & & & PULSES & Tstart(NPULSE) \\
\hline 3 & $\mathrm{U}^{*} 4$ & & & PULSES & Qual(NPULSE) \\
\hline 4 & $\mathrm{R}^{*} 4$ & 16 & {$[-100 ., 555.3$} & PULSES & FITPED(NPULSE) \\
\hline 5 & $\mathrm{R}^{*} 4$ & 16 & {$[-50 ., 605.35$} & PULSES & FITMAX(NPULSE) \\
\hline 6 & $\mathrm{R}^{*} 4$ & & & PULSES & FITTIME(NPULSE) \\
\hline 7 & $\mathrm{R}^{*} 4$ & & & PULSES & FITCHI2(NPULSE) \\
\hline 1 & $I^{*} 4$ & & {$[0,500]$} & PULSESL & NPULSE1 \\
\hline 2 & $\mathrm{U}^{*} 4$ & 16 & & PULSESL & NSAM(NPULSE1) \\
\hline 3 & $\mathrm{U}^{*} 4$ & 8 & {$[0,255]$} & PULSESL & DISC(NPULSE1) \\
\hline 4 & $\mathrm{U}^{*} 4$ & 1 & {$[0,1]$} & PULSESL & Phase(NPULSE1) \\
\hline 5 & $\mathrm{U}^{*} 4$ & 9 & {$[0,511]$} & PULSESL & MaxV(NPULSE1) \\
\hline 6 & $\mathrm{U}^{*} 4$ & 14 & {$[0,10000]$} & PULSESL & IMAXV(NPULSE1) \\
\hline 7 & $\mathrm{U}^{*} 4$ & 8 & {$[0,255]$} & PULSESL & Ped(NPULSE1) \\
\hline 8 & $\mathrm{U}^{*} 4$ & 9 & {$[0,500]$} & PULSESL & IFSDPUL(NPULSE1) \\
\hline 9 & $\mathrm{U}^{*} 4$ & 3 & {$[0,6]$} & PULSESL & NFSDHIT(NPULSE1) \\
\hline 10 & $\mathrm{U}^{*} 4$ & 9 & {$[0,500]$} & PULSESL & ISDATA(NPULSE1) \\
\hline 11 & $\mathrm{U}^{*} 4$ & 8 & {$[0,255]$} & PULSESL & ISAM(8,NPULSE1) \\
\hline 1 & $I^{*} 4$ & & {$[0,500]$} & SLIMDATA & NSDATA \\
\hline 2 & $\mathrm{R}^{*} 4$ & & & SLIMDATA & TimeS(NSDATA) \\
\hline 3 & $\mathrm{R}^{*} 4$ & 16 & {$[-100 ., 555.3$} & SLIMDATA & MaxS(NSDATA) \\
\hline 4 & $\mathrm{R}^{*} 4$ & 16 & {$[-100,, 555.3$} & SLIMDATA & PedS(NSDATA) \\
\hline 5 & $\mathrm{R}^{*} 4$ & & & SLIMDATA & Chi2S(NSDATA) \\
\hline
\end{tabular}

Table A.2: PULSES, PULSESL and SLIMDATA data blocks of the G2OFF decay electron ntuple data structure. These data blocks contain information regarding pulses extracted from the WFD calorimeter readout. 


\begin{tabular}{|c|c|c|c|c|c|}
\hline \multicolumn{6}{|c|}{ G2OFF Ntuple ID=10, T0, Laser \& Calo MTDC Data Blocks } \\
\hline Var numb & Type & Packing & Range & Block & Name \\
\hline 1 & $\mathrm{U}^{*} 4$ & 16 & & TOPULSE & T0NSAM \\
\hline 2 & $\mathrm{R}^{*} 4$ & & & TOPULSE & T0start \\
\hline 3 & $\mathrm{R}^{*} 4$ & & & TOPULSE & T0mean \\
\hline 4 & $\mathrm{U}^{*} 4$ & 10 & & PSD2 & YPAT(NPSD2) \\
\hline 5 & $\mathrm{U}^{*} 4$ & {$[0,512]$} & TOPULSE & T0MaxV & \\
\hline 5 & $\mathrm{R}^{*} 4$ & 16 & {$[0 ., 65535]$.} & TOPULSE & T0Area \\
\hline 6 & $\mathrm{U}^{*} 4$ & 8 & {$[0,255]$} & TOPULSE & T0Ped \\
\hline 7 & $\mathrm{R}^{*} 4$ & 16 & {$[0 ., 655.35]$} & TOPULSE & ToWidth \\
\hline 1 & $\mathrm{U}^{*} 4$ & 16 & & LPULSE & LNSAM \\
\hline 2 & $\mathrm{U}^{*} 4$ & 10 & {$[0,512]$} & LPULSE & LMaxV \\
\hline 3 & $\mathrm{R}^{*} 4$ & & & LPULSE & Lstart \\
\hline 4 & $\mathrm{R}^{*} 4$ & & & LPULSE & LOffset \\
\hline 5 & $\mathrm{U}^{*} 4$ & 8 & {$[0,255]$} & LPULSE & LPed \\
\hline 6 & $\mathrm{U}^{*} 4$ & & & LPULSE & LQual \\
\hline 7 & $\mathrm{R}^{*} 4$ & 16 & {$[-100 ., 555.3$} & LPULSE & LFPED \\
\hline 8 & $\mathrm{R}^{*} 4$ & 16 & {$[-100 ., 555.3$} & LPULSE & LFMAX \\
\hline 9 & $\mathrm{R}^{*} 4$ & & & LPULSE & LFTIME \\
\hline 10 & $\mathrm{R}^{*} 4$ & & & LPULSE & LFCHI2 \\
\hline 1 & $I^{*} 4$ & & {$[0,500]$} & CALMTDC & NMTDCST \\
\hline 2 & $\mathrm{R}^{*} 4$ & & & CALMTDC & TDMTDC(NMTDCST) \\
\hline 3 & $\mathrm{U}^{*} 4$ & 13 & {$[0,8000]$} & CALMTDC & MTPULSE(NMTDCST) \\
\hline
\end{tabular}

Table A.3: TOPULSE, LPULSE and CALMTDC data blocks of the G2OFF decay electron ntuple data structure. These data blocks contain information regarding the T0 and laser pulses, as well as the calorimeter MTDC timing readouts. 


\begin{tabular}{|c|c|c|c|c|c|}
\hline \multicolumn{6}{|c|}{ G2OFF Ntuple ID=10, Kicker, FSD \& PSD Data Blocks } \\
\hline Var numb & Type & Packing & Range & Block & Name \\
\hline 1 & $\mathrm{R}^{*} 4$ & & & "KICKER & FAKPhase(3) \\
\hline 2 & $\mathrm{R}^{*} 4$ & & & KICKER & FAKAmp $(3)$ \\
\hline 3 & $\mathrm{R}^{*} 4$ & & & KICKER & FAKFreq(3) \\
\hline 4 & $\mathrm{R}^{*} 4$ & & & KICKER & FAKDecay $(3)$ \\
\hline 5 & $\mathrm{R}^{*} 4$ & & & KICKER & FAKChiSq $(3)$ \\
\hline 1 & $\mathrm{I}^{*} 4$ & & {$[0,600]$} & PSD2 & NPSD2 \\
\hline 2 & $\mathrm{R}^{*} 4$ & & & PSD2 & TPSD2(NPSD2) \\
\hline 3 & $\mathrm{U}^{*} 4$ & & & PSD2 & XPAT(NPSD2) \\
\hline 4 & $\mathrm{U}^{*} 4$ & & & PSD2 & YPAT(NPSD2) \\
\hline 5 & $\mathrm{U}^{*} 4$ & 13 & {$[0,8000]$} & PSD2 & PPULSE(NPSD2) \\
\hline 1 & $\mathrm{I}^{*} 4$ & & {$[0,600]$} & PSDT & NPSDT \\
\hline 2 & $\mathrm{R}^{*} 4$ & & & PSDT & TPSDT(NPSDT) \\
\hline 1 & $I^{*} 4$ & & {$[0,1000]$} & FSDPULSE & NFSDPUL \\
\hline 2 & $\mathrm{U}^{*} 4$ & 3 & {$[0,5]$} & FSDPULSE & FSDFing(NFSDPUL) \\
\hline 3 & $\mathrm{R}^{*} 4$ & 16 & {$[-30 ., 35.535$} & FSDPULSE & FSDFingT(NFSDPUL) \\
\hline 1 & $I^{*} 4$ & & {$[0,1200]$} & PSD2PC & NPSD2PC \\
\hline 2 & $\mathrm{R}^{*} 4$ & & & PSD2PC & TPSD2PC(NPSD2PC) \\
\hline 1 & $\mathrm{I}^{*} 4$ & & {$[0,1200]$} & PSDA & NPSDA \\
\hline 2 & $\mathrm{R}^{*} 4$ & & & PSDA & TPSDA(NPSDA) \\
\hline
\end{tabular}

Table A.4: KICKER, PSD2, PSDT, FSDPULSE, PSD2PC and PSDA data blocks of the G2OFF decay electron ntuple data structure. These data blocks contain information regarding the kicker WFD, PSD and FSD MTDC readouts. 


\section{Appendix B}

\section{The Ratio Method - Derivations}

\section{B.1 Simple 3-Parameter Function}

This is derived from the simple 5-parameter function for $\mathrm{N}(\mathrm{t})$,

$$
N_{5}(t)=N_{0} e^{-t / \tau}\left(1+A \cos \left(\omega_{a} t\right)\right)
$$

where we have set the $g$ - 2 phase, $\phi_{a}$, equal to zero for simplicity (it will of course be added in later).

Let

$$
u_{+}(t)=N_{5}(t+T / 2)=N_{0} e^{-t / \tau} e^{-T / 2 \tau}\left(1-A \cos \left(\omega_{a} t+\delta\right)\right)
$$

and

$$
u_{-}(t)=N_{5}(t-T / 2)=N_{0} e^{-t / \tau} e^{T / 2 \tau}\left(1-A \cos \left(\omega_{a} t-\delta\right)\right)
$$

where $T$ is a good approximation of the $g-2$ period (to about 5-10 ppm) and $\delta$ is the difference between $T$ and $T_{a_{\text {true }}}, \delta=\frac{\omega_{a}}{2}\left(T-T_{a_{\text {true }}}\right)=\pi \times \delta T$.

Now let

$$
\begin{aligned}
& v_{1}(t)=v_{2}(t)=N_{5}(t) \\
& U(t)=u_{+}(t)+u_{-}(t) \\
& V(t)=v_{1}(t)+v_{2}(t) \\
& r(t)=\frac{V(t)-U(t)}{V(t)+U(t)}
\end{aligned}
$$

Expanding $e^{ \pm T / 2 \tau}$ and $A \cos \left(\omega_{a} t \pm \delta\right)$, we find

$$
\begin{aligned}
u_{+}(t)= & N_{0} e^{-t / \tau}\left(1-\frac{1}{2} \frac{T}{\tau}+\frac{1}{2}\left(\frac{1}{2} \frac{T}{\tau}\right)^{2}-\ldots\right) \times \\
& \left(1-A \cos \left(\omega_{a} t\right)+A \delta \sin \left(\omega_{a} t\right)\right)
\end{aligned}
$$




$$
\begin{aligned}
u_{-}(t)= & N_{0} e^{-t / \tau}\left(1+\frac{1}{2} \frac{T}{\tau}+\frac{1}{2}\left(\frac{1}{2} \frac{T}{\tau}\right)^{2}+\ldots\right) \times \\
& \left(1-A \cos \left(\omega_{a} t\right)-A \delta \sin \left(\omega_{a} t\right)\right)
\end{aligned}
$$

and so,

$$
\begin{aligned}
U(t)= & 2 N_{0} e^{-t / \tau}\left(1+\frac{1}{2}\left(\frac{1}{2} \frac{T}{\tau}\right)^{2}\right)\left(1-A \cos \left(\omega_{a} t\right)\right)- \\
& N_{0} e^{-t / \tau} \frac{T}{\tau} A \delta \sin \left(\omega_{a} t\right)
\end{aligned}
$$

Now let

$$
C_{1}=\frac{1}{16}\left(\frac{T}{\tau}\right)^{2} \simeq 2.87 \times 10^{-4}
$$

and

$$
C_{2}=\frac{T A \delta}{4 \tau} \simeq 2 \times 10^{-7}
$$

then,

$$
\begin{gathered}
V(t)-U(t)=4 N_{0} e^{-t / \tau}\left(A \cos \left(\omega_{a} t\right)+C_{2} \sin \left(\omega_{a} t\right)\right)- \\
4 N_{0} e^{-t / \tau} C_{1}\left(1-A \cos \left(\omega_{a} t\right)\right)
\end{gathered}
$$

where $A^{\prime}=A\left(1+C_{1}\right)$

Since the terms in the denominator are small, we can rewrite this as

$$
\begin{aligned}
r(t)= & \left(A^{\prime} \cos \left(\omega_{a} t\right)-C_{1}+C_{2} \sin \left(\omega_{a} t\right)\right) \times \\
& \left(1-C_{1}\left(1-A \cos \left(\omega_{a} t\right)\right)+C_{2} \sin \left(\omega_{a} t\right)\right)
\end{aligned}
$$


Keeping terms to first order, we have

$$
\begin{aligned}
r(t)= & A^{\prime} \cos \left(\omega_{a} t\right)-C_{1}+C_{2} \sin \left(\omega_{a} t\right)-C_{1} A^{\prime} \cos \left(\omega_{a} t\right)+ \\
& C_{1} A A^{\prime} \cos ^{2}\left(\omega_{a} t\right)+C_{2} A^{\prime} \sin \left(\omega_{a} t\right) \cos \left(\omega_{a} t\right) \\
= & A^{\prime \prime} \cos \left(\omega_{a} t\right)-C_{1}+C_{2} \sin \left(\omega_{a} t\right)+ \\
& C_{2} A^{\prime} \cos \left(\omega_{a} t\right) \sin \left(\omega_{a} t\right)+C_{1} A A^{\prime} \cos ^{2}\left(\omega_{a} t\right)
\end{aligned}
$$

where

$$
A^{\prime \prime}=A^{\prime}\left(1-C_{1}\right)=A\left(1-C_{1}^{2}\right)
$$

Terms involving $C_{2}$ are of order $10^{-7}$ or less, and so may be neglected, and we are left with

$$
r(t)=A^{\prime \prime} \cos \left(\omega_{a} t\right)-C_{1}+C_{1} A A^{\prime} \cos ^{2}\left(\omega_{a} t\right)
$$

In practice, the last term is omitted, so that

$$
r(t)=A \cos \left(\omega_{a} t\right)-C_{1}
$$

\section{B.2 Derivation of the Ratio Function with a Background Term}

Here we assume a background term $B(t)$ such that

$$
N(t)=N_{5}(t)+B(t)
$$

where $B(t) / N_{5}(t) \ll 1$. Eq. B.15 from the previous derivation is then modified to be

$$
\begin{aligned}
r(t) & =\frac{\left.A \cos \left(\omega_{a} t\right)+C_{2} \sin \left(\omega_{a} t\right)\right)-C_{1}\left(1-A \cos \left(\omega_{a} t\right)\right)+b_{\text {num }}(t)}{\left(1-C_{2} \sin \left(\omega_{a} t\right)\right)+C_{1}\left(1-A \cos \left(\omega_{a} t\right)\right)+b_{\text {den }}(t)} \\
& =\frac{A^{\prime} \cos \left(\omega_{a} t\right)-C_{1}+C_{2} \sin \left(\omega_{a} t\right)+b_{\text {num }}(t)}{1-C_{2} \sin \left(\omega_{a} t\right)+C_{1}\left(1-A \cos \left(\omega_{a} t\right)\right)+b_{\text {den }}(t)}
\end{aligned}
$$

where

$$
b_{\text {num }}(t)=\frac{2 B(t)-B(t+T / 2)-B(t-T / 2)}{4 N_{0} \exp (-t / \tau)}
$$

and

$$
b_{\text {den }}(t)=\frac{2 B(t)+B(t+T / 2)+B(t-T / 2)}{4 N_{0} \exp (-t / \tau)}
$$


Therefore, the ratio becomes, neglecting the same terms as before but keeping the background terms to first order,

$$
r(t)=A \cos \left(\omega_{a} t\right)\left(1-b_{\text {den }}(t)\right)-C_{1}+b_{\text {num }}(t)
$$

From this result one immediately sees that if the background term is large enough, the fit result for $\omega_{a}$ will be fit-start-time dependent. We also see that any background that oscillates, such as pileup and CBO, will produce "beat frequencies" in the ratio time spectrum. Pileup will produce beat frequencies of $f_{\text {beat }}=0$ and $f=2 f_{a}$, whereas CBO will produce beat frequencies of $f_{\text {beat }}=f_{\mathrm{CBO}} \pm f_{a}$. If a beat frequency sits close to the actual $g$-2 frequency $f_{a}$, then fits to the data that do not have background subtracted or fits to the data with a simple three-parameter function will show oscillations in the fit value of $\omega_{a}$ at frequencies $f=f_{\text {beat }} \pm f_{a}$. This is readily seen when the 2001 data set is fit to the three-parameter ratio function: $\omega_{a}$ oscillates vs. fit start time at frequencies $f \simeq 40 \mathrm{kHz}$ and $f \simeq 32 \mathrm{kHz}$ for the low- and high-n data sets respectively.

Another, perhaps more subtle, expectation is $g$-2 oscillations in difference plots of $\omega$ vs. fit start time with a non-oscillating background "removed" (either subtracted or fitted out) and the background left in. A good example of this is gain or ESC corrections; since gain or ESC effects $N_{0}, \tau$ and in particular $A$ versus time, one finds that $b_{d e n}(t) \propto \cos \left(\omega_{a} t\right)$. Therefore, in the fit-start-time scan of $\omega_{a}$ with the background left in, $\omega_{a}$ will have small oscillations at $f=2 f_{a}$ (since $\cos \left(\omega_{a}\right)^{2}=\frac{1}{2}\left(1+\cos \left(2 \omega_{a}\right)\right)$ ) and the fit-start-time scan of $\omega_{a}$ with the background term removed will either have no oscillations at this frequency or at least oscillations of a much smaller amplitude. When looking at $(R($ corr. $)$ vs. $t)-(R$ (no corr.) vs. $t)$, one expects to see oscillations at twice the $g$-2 frequency. This is indeed the case, as shown in Fig. 4.49.

\section{B.3 A General Derivation of the Ratio Function with CBO}

From past experience, we know that Eq. B.1 is not sufficient to obtain stable fit results from the data. This is because the counting rate is changed by other effects, primarily those from detector gains, muon losses, and coherent betatron oscillations (CBO). The ratio method is largely insensitive to detector gains and muon losses, however it is not blind to CBO.

The number, asymmetry and phase are affected by CBO, and therefore the number of decay electrons or positrons as a function of time is rewritten as:

$$
N(t)=\left(N_{0}+N_{\mathrm{CBO}}(t)\right) e^{-t / \tau}\left[1+\left(A+A_{\mathrm{CBO}}(t)\right) \cos \left(\omega_{a} t+\phi+\phi_{\mathrm{CBO}}(t)\right)\right]
$$

Assuming that the effect of CBO is small, we expand Eq. B.26 out and keep terms to only first order:

$$
\begin{aligned}
N(t)= & \left(N_{0}+N_{\mathrm{CBO}}(t)\right) e^{-t / \tau}\left[1+A \cos \left(\omega_{a} t+\phi\right)+A_{\mathrm{CBO}}(t) \cos \left(\omega_{a} t+\phi\right)-\right. \\
& \left.A \phi_{\mathrm{CBO}}(t) \sin \left(\omega_{a} t+\phi\right)\right]
\end{aligned}
$$




$$
\begin{aligned}
= & N_{0} e^{-t / \tau}\left(1+A \cos \left(\omega_{a} t+\phi\right)\right)\left(1+\frac{N_{\mathrm{CBO}}(t)}{N_{0}}\right)+ \\
& N_{0} e^{-t / \tau}\left(1+\frac{N_{\mathrm{CBO}}(t)}{N_{0}}\right)\left(A_{\mathrm{CBO}}(t) \cos \left(\omega_{a} t+\phi\right)\right)- \\
& \left.A \phi_{\mathrm{CBO}}(t) \sin \left(\omega_{a} t+\phi\right)\right) \\
= & N_{5}(t)\left(1+f_{1}(t)+f_{2}(t)\right) \\
= & N_{5}(t)\left(1+f_{\mathrm{CBO}}(t)\right)
\end{aligned}
$$

where

$$
\begin{gathered}
f_{1}(t)=\frac{N_{\mathrm{CBO}}(t)}{N_{0}} \\
f_{2}(t)=\frac{\left.A_{\mathrm{CBO}}(t) \cos \left(\omega_{a} t+\phi\right)\right)-A \phi_{\mathrm{CBO}}(t) \sin \left(\omega_{a} t+\phi\right)}{1+A \cos \left(\omega_{a} t+\phi\right)}
\end{gathered}
$$

and

$$
f_{\mathrm{CBO}}(t)=f_{1}(t)+f_{2}(t)
$$

Eq. B.27 is equivalent to having a background term,

$$
N(t)=N_{5}(t)+B(t)
$$

where

$$
B(t)=N_{5}(t) \cdot f_{\mathrm{CBO}}(t)
$$

In the previous section it was shown that with a background term $B(t)$, Eq. B.20 becomes:

$$
r(t)=\left[1-b_{\text {den }}(t)\right] A \cos \left(\omega_{a} t+\phi\right)-C_{1}+b_{\text {num }}(t)
$$

where

$$
\begin{gathered}
b_{\text {den }}(t)=2 b(t)+b_{+}(t)+b_{-}(t), \\
b_{\text {num }}(t)=2 b(t)-b_{+}(t)-b_{-}(t), \\
b(t)=\frac{B(t)}{4 N_{0} \exp (-t / \tau)}, \\
b_{+}(t)=b(t+T / 2),
\end{gathered}
$$

and

$$
b_{-}(t)=b(t-T / 2)
$$

Now,

$$
\begin{aligned}
b_{+}(t)+b_{-}(t)= & \frac{1}{4}\left(1-\frac{T}{2 \tau}+\frac{1}{2}\left(\frac{T}{2 \tau}\right)^{2}\right)\left(1-A \cos \left(\omega_{a} t\right)\right) \times \\
& f_{\mathrm{CBO}}(t+T / 2)+
\end{aligned}
$$




$$
\begin{aligned}
& \frac{1}{4}\left(1+\frac{T}{2 \tau}+\frac{1}{2}\left(\frac{T}{2 \tau}\right)^{2}\right)\left(1-A \cos \left(\omega_{a} t\right)\right) \times \\
& f_{\mathrm{CBO}}(t-T / 2)
\end{aligned}
$$

where we have left out all terms with $A \delta \sin ()$ since they are small compared to the $A \cos ()$ terms, and the phase, $\phi$ is implied in all cosine terms. Since $f_{\mathrm{CBO}}(t)$ is small, we keep only the highest order terms:

$$
b_{+}(t)+b_{-}(t)=\frac{1}{4}\left(1-A \cos \left(\omega_{a} t\right)\right)\left(f_{\mathrm{CBO}}(t+T / 2)+f_{\mathrm{CBO}}(t-T / 2)\right)
$$

Similarly,

$$
2 b(t)=\frac{1}{2}\left(1+A \cos \left(\omega_{a} t\right)\right) f_{\mathrm{CBO}}(t)
$$

Using Eqs. B.28 and B.29, we find that

$$
\begin{aligned}
b_{+}(t)+b_{-}(t)= & \frac{1}{4}\left(1-A \cos \left(\omega_{a} t\right)\right)\left(\frac{N_{\mathrm{CBO}}(t+T / 2)+N_{\mathrm{CBO}}(t-T / 2)}{N_{0}}\right)- \\
& \frac{1}{4}\left(A_{\mathrm{CBO}}(t+T / 2)+A_{\mathrm{CBO}}(t-T / 2)\right) \cdot \cos \left(\omega_{a} t\right)+ \\
& \frac{1}{4}\left(A \phi_{\mathrm{CBO}}(t+T / 2)+A \phi_{\mathrm{CBO}}(t-T / 2)\right) \cdot \sin \left(\omega_{a} t\right)
\end{aligned}
$$

and again similarly,

$$
2 b(t)=\frac{1}{2}\left(\left(1+A \cos \left(\omega_{a} t\right)\right) \frac{N_{\mathrm{CBO}}(t)}{N_{0}}+A_{\mathrm{CBO}}(t) \cos \left(\omega_{a} t\right)-A \phi_{\mathrm{CBO}}(t) \sin \left(\omega_{a} t\right)\right)
$$

Next, we look at $b_{\text {den }}$ and $b_{\text {num }}$ :

$$
\begin{aligned}
b_{d e n}= & 2 b(t)+b_{+}+b_{-} \\
= & \frac{1}{4}\left(\frac{2 N_{\mathrm{CBO}}+N_{c b o+}+N_{c b o-}}{N_{0}}\right)+ \\
& \frac{1}{4}\left(A \cos \left(\omega_{a} t\right) \frac{2 N_{\mathrm{CBO}}-N_{c b o+}-N_{c b o-}}{N_{0}}\right)+ \\
& \frac{1}{4}\left(\left(2 A_{\mathrm{CBO}}-A_{c b o+}-A_{c b o-}\right) \cos \left(\omega_{a} t\right)\right)- \\
& \frac{1}{4}\left(\left(2 A \phi_{\mathrm{CBO}}-A \phi_{c b o+}-A \phi_{c b o-}\right) \sin \left(\omega_{a} t\right)\right)
\end{aligned}
$$

and

$$
\begin{aligned}
b_{\text {num }}= & 2 b(t)-b_{+}-b_{-} \\
= & \frac{1}{4}\left(\frac{2 N_{\mathrm{CBO}}-N_{c b o+}-N_{c b o-}}{N_{0}}\right)+ \\
& \frac{1}{4}\left(A \cos \left(\omega_{a} t\right) \frac{2 N_{\mathrm{CBO}}+N_{c b o+}+N_{c b o-}}{N_{0}}\right)+
\end{aligned}
$$




$$
\begin{aligned}
& \frac{1}{4}\left(\left(2 A_{\mathrm{CBO}}+A_{c b o+}+A_{c b o-}\right) \cos \left(\omega_{a} t\right)\right)- \\
& \frac{1}{4}\left(\left(2 A \phi_{\mathrm{CBO}}+A \phi_{c b o+}+A \phi_{c b o-}\right) \sin \left(\omega_{a} t\right)\right)
\end{aligned}
$$

The above equations look a bit scary, but we can simplify them quite a bit. If one assumes $N_{\mathrm{CBO}} \sim A_{\mathrm{CBO}} \sim \phi_{\mathrm{CBO}} \sim \exp \left(-t / \tau_{\mathrm{CBO}}\right) \cos \left(\omega_{\mathrm{CBO}} t\right)$ and that $\tau_{\mathrm{CBO}}$ is much greater than $T / 2$, then since

$$
\cos \left(\omega_{\mathrm{CBO}}(t \pm T / 2)\right)=\cos \left(\omega_{\mathrm{CBO}} t\right) \cos \left(\omega_{\mathrm{CBO}} T / 2\right) \mp \sin \left(\omega_{\mathrm{CBO}} t\right) \sin \left(\omega_{\mathrm{CBO}} T / 2\right)
$$

we find that $N_{c b o+}+N_{c b o-} \simeq 2 N_{\mathrm{CBO}} \cos \left(\omega_{\mathrm{CBO}} T / 2\right), A_{c b o+}+A_{c b o-} \simeq 2 A_{\mathrm{CBO}} \cos \left(\omega_{\mathrm{CBO}} T / 2\right)$ and $\phi_{c b o+}+\phi_{c b o-} \simeq 2 \phi_{\mathrm{CBO}} \cos \left(\omega_{\mathrm{CBO}} T / 2\right)$. Therefore we can rewrite Eqs. B.44 and B.45 as

$$
\begin{aligned}
b_{d e n}= & \frac{1}{2}\left(\frac{N_{\mathrm{CBO}}}{N_{0}}(1+\xi)+A \cos \left(\omega_{a} t\right) \frac{N_{\mathrm{CBO}}}{N_{0}}(1-\xi)+\right. \\
& \left.A_{\mathrm{CBO}}(1-\xi) \cos \left(\omega_{a} t\right)-\phi_{\mathrm{CBO}}^{\prime}(1-\xi) \sin \left(\omega_{a} t\right)\right)
\end{aligned}
$$

and

$$
\begin{aligned}
b_{\text {num }}= & \frac{1}{2}\left(\frac{N_{\mathrm{CBO}}}{N_{0}}(1-\xi)+A \cos \left(\omega_{a} t\right) \frac{N_{\mathrm{CBO}}}{N_{0}}(1+\xi)+\right. \\
& \left.A_{\mathrm{CBO}}(1+\xi) \cos \left(\omega_{a} t\right)-\phi_{\mathrm{CBO}}^{\prime}(1+\xi) \sin \left(\omega_{a} t\right)\right)
\end{aligned}
$$

where $\xi=\cos \left(\omega_{\mathrm{CBO}} T / 2\right)$ and $\phi_{\mathrm{CBO}}^{\prime}=A \phi_{\mathrm{CBO}}$.

Several aspects of these results should be noted. First, when we took data with quadrupole focusing such that $T_{\mathrm{CBO}} \simeq \frac{1}{2} T_{a}$ (ie: 1999 and 2000 runs), then many terms in $b_{\text {den }}$ and $b_{\text {num }}$ cancel since $\xi \simeq 1$. We end up with

$$
b_{\text {den }} \simeq \frac{N_{\mathrm{CBO}}}{N_{0}}
$$

and

$$
\begin{aligned}
b_{\text {num }} \simeq & \left(A \cos \left(\omega_{a} t\right) \frac{N_{\mathrm{CBO}}}{N_{0}}\right)+ \\
& A_{\mathrm{CBO}}^{\prime} \cos \left(\omega_{a} t\right)-\phi_{\mathrm{CBO}}^{\prime} \sin \left(\omega_{a} t\right)
\end{aligned}
$$

Looking at Eq. B.33 we see that all CBO terms involve products of $\cos \left(\omega_{\mathrm{CBO}} t\right)$ with either $\cos \left(\omega_{a} t\right)$ or $\sin \left(\omega_{a} t\right)$, which reduce to some linear combination of $\cos \left(\left(\omega_{\mathrm{CBO}}-\omega_{a}\right) t\right)$ and $\cos \left(\left(\omega_{\mathrm{CBO}}+\omega_{a}\right) t\right)$. Note that there are no terms involving simply $\cos \left(\omega_{\mathrm{CBO}} t\right)$ ! This is a rather unique result, and obviously does not apply to the 2001 data.

Next, the amplitude of the main cbo peak can in principle be either minimally zero or maximally the same size as would be seen in a multi-parameter fit of the normal "wiggle" plot, which is the special case of the 2000 run conditions. Otherwise, the cbo amplitude should be suppressed by some factor in the ratio method. For the 2001 data, where $f_{\mathrm{CBO}} \simeq$ 419 and $490 \mathrm{kHz}$, then $\xi \simeq-0.15$ and 0.10 respectively and the suppression factor is $\sim 2$. 


\section{B.4 Error Propagation}

In a counting measurement, the uncertainty in the number of counts within a time bin is simply the square root of the number of counts in that time bin, or, more precisely the square root of the function averaged over that time bin. Therefore, if one were to fit the data directly to a function $N(t)$, in a log-likelihood or $\chi^{2}$ fit, the error on each bin is $\sqrt{N}$ or $\sqrt{N(t)}$. However, when fitting the data to a function $f(N(t))$, the uncertainty of that function must be determined.

The ratio is defined as

$$
r(t)=\frac{V(t)-U(t)}{V(t)+U(t)}
$$

where

$$
V(t)=v_{1}(t)+v_{2}(t)=N_{1}(t)+N_{2}(t)
$$

and

$$
U(t)=u_{+}(t)+u_{-}(t)=N(t+T / 2)+N(t-T / 2)
$$

It is important to note that $N_{1}(t), N_{2}(T), N(t+T / 2), N(t-T / 2)$ are all statistically independent data sets. The uncertainty of the ratio at time $t$ is therefore

$$
\sigma_{r}(t)^{2}=\left(\frac{\partial r(t)}{\partial V(t)}\right)^{2} \delta V(t)^{2}+\left(\frac{\partial r(t)}{\partial U(t)}\right)^{2} \delta U(t)^{2}
$$

Now,

$$
\begin{aligned}
& \frac{\partial r(t)}{\partial V}=\frac{1}{U+V}-\left(\frac{V-U}{(U+V)^{2}}\right), \\
& \frac{\partial r(t)}{\partial U}=\frac{-1}{U+V}-\left(\frac{V-U}{(U+V)^{2}}\right), \\
& \delta V^{2}=\delta v_{1}^{2}+\delta v_{2}^{2}=v_{1}+v_{2}=V
\end{aligned}
$$

and

$$
\delta U^{2}=\delta u_{+}^{2}+\delta u_{-}^{2}=u_{+}+u_{-}=U
$$

where both $V$ and $U$ are at time $t$. Therefore we find

$$
\sigma_{r}^{2}=\frac{4 U V}{(V+U)^{3}}=\frac{1-r^{2}}{V+U}
$$

The above is the correct derivation of the error propagation when no backgrounds are subtracted from the data. However, in this analysis, pileup events are statistically constructed from the data and then subtracted from the data set; therefore one must properly calculate the correct error bar due to subtracting a subset of correlated data from a set of data. This is determined in [118] for a multi-parameter fit: the error at a given time $t$ is increased by

$$
\sigma_{N}^{2} \rightarrow \sigma_{N}^{2}\left(1+\gamma e^{\left(t-t_{0}\right) / \tau}\right)
$$

where $\gamma$ is the product of the fraction of pileup events at $t_{0}$ and a "correlation" factor as described in [118], and $\tau$ is the pileup lifetime $(32.2 \mu \mathrm{s}) . \gamma$ was determined for each detector 


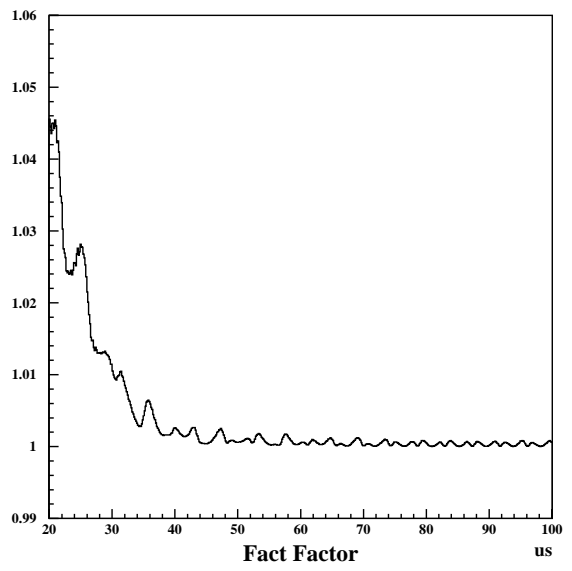

Figure B.1: The "fast factor" as a function of time after injection. This enhances the effect of pileup at early times, albeit by a small amount.

in this data set at $t_{0}=32 \mu \mathrm{s}$; the typical value of $\gamma$ is $\sim 1.04 \%$.

However, one must first determine the proper functional form of the "correct" error of the ratio due to pileup subtraction. We begin with the modification of the errors on $V$ and $U$.

$$
\begin{aligned}
\delta V^{2} & =\delta v_{1}^{2}(1+\gamma(t))+\delta v_{2}^{2}(1+\gamma(t)) \\
& =\left(v_{1}+v_{2}\right)(1+\gamma(t))=V(1+\gamma(t))
\end{aligned}
$$

where $\gamma(t)=\gamma e^{\left(t-t_{0}\right) / \tau}$, and

$$
\begin{aligned}
\delta U^{2} & =\delta u_{+}^{2}\left(1+\gamma\left(t_{+}\right)\right)+\delta u_{-}^{2}\left(1+\gamma\left(t_{-}\right)\right) \\
& =u_{+}\left(1+\gamma(t) e^{-T / 2 \tau}\right)+u_{-}\left(1+\gamma(t) e^{T / 2 \tau}\right) \\
& \simeq\left(u_{+}+u_{-}\right)\left(1+\gamma(t)\left(1+\frac{1}{2}\left(\frac{T}{2 \tau}\right)^{2}\right)\right) \\
& =U\left(1+\gamma(t)\left(1+\frac{1}{2}\left(\frac{T}{2 \tau}\right)^{2}\right)\right)
\end{aligned}
$$

However, since $\gamma(t)$ is already very small at $32 \mu \mathrm{s}$, we neglect the extra $\frac{1}{2}\left(\frac{T}{2 \tau}\right)^{2}$ term and find that the change in the error due to pileup subtraction is, to a very good approximation, the same for ratio fits as for multi-parameter wiggle fits: $\sigma_{r}^{2} \rightarrow \sigma_{r}^{2}\left(1+\gamma e^{-\left(t-t_{0}\right) / \tau}\right)$.

One further correction must be made, and that is to the value of $\gamma$ itself. At early times after injection, the bunched structure of the beam causes an enhancement of the pileup by the so-called fast-factor:

$$
f f(t)=\frac{\left\langle N^{2}\right\rangle(t)}{\langle N\rangle^{2}(t)}
$$

where $t$ denotes the center of a bin of a given bin width $d t$. The fast factor was found by extracting $N(t)$ spectra using bin widths of $d t=5 \mathrm{~ns}$, and taking the ratio of $N(t)^{2}$ 
re-binned with $d t=150 \mathrm{~ns}$ to the square of $N(t)$ re-binned with $d t=150 \mathrm{~ns}$. In the 2000 data, the fast factor was found to be as large as 1.6 around $20 \mu$ s after injection, however, as shown in Fig. B.1, in the 2001 data the fast factor is only $~ 1.03$ at $20 \mu \mathrm{s}$. The difference is due to a sharp reduction in data rate between the 2000 and 2001 runs.

We therefore find that the error on the ratio at time $t$ is given as $\sigma_{r} \rightarrow \sigma_{r}(1+$ $\left.f f(t) \gamma e^{-\left(t-t_{0}\right) / \tau}\right)$. Using this improved estimation of the error bars of the ratio results in an improvement in the $\chi^{2} /$ d.o.f. of the 9 -parameter fit by about $3 \times 10^{-3}$, changes the fitted $R$ value by less than $0.06 \mathrm{ppm}$, and changes the statistical uncertainty on $R$ by less than $0.005 \mathrm{ppm}$. 


\section{Journal Abbreviations}

Ann. of Phys. . . . . . . . . . . . . . . Annals of Physics

Eur. Phys. J. . . . . . . . . . . . . . The European Physical Journal Nucl. Phys. . . . . . . . . . . . . . . . . . . Nuclear Physics Nucl. Inst. . . . . . . . . . . . . . Nuclear Instruments and Methods Phil. Mag. . . . . . . . . . . . . . . . . Philosophical Magazine Phys. Rev. . . . . . . . . . . . . . . . . Physical Review Phys. Rev. Lett. . . . . . . . . . . . . . . Physical Review Letters Rev. Mod. Phys. . . . . . . . . . . . . Review of Modern Physics Roy. Soc. Proc. . . . . . . . . . . . . . . . . The Royal Society Publications Z. Physik . . . . . . . . . . . . . . . . Zeitschrift Für Physik ZhETF . . . . . . . . . Journal of Experimental and Theoretical Physics 


\section{Bibliography}

[1] W. Gerlach and O. Stein, Z. Physik 9, 353 (1922).

[2] S. Goudsmit and G. E. Uhlenbeck, Naturwissenshaften 13, 953 (1925).

[3] T. E. Phipps and J. B. Taylor, Phys. Rev. 29, 309 (1927).

[4] P. A. M. Dirac, Roy. Soc. Proc. 117, 610 (1928).

[5] I. Estermann and O. Stern, Z. Physik 85, 17 (1933).

[6] P.Kusch and H. M. Foley, Phys. Rev. 74, 250 (1948).

[7] J. S. Schwinger, Phys. Rev. 73, 416 (1948).

[8] A. Czarnecki and W. J. Marciano, Phys. Rev. D64, (2001).

[9] S. Laporta, Phys. Rev. D47, 4793 (1993).

[10] M. Knecht, hep-ph/0307239 (2003).

[11] R. S. van Dyck Jr, P. B. Swchinberg, and H. G. Dehmelt, Phys. Rev. Lett. 26, 26 (1987).

[12] P. J. Mohr and B. N. Taylor, Rev. Mod. Phys. 72, 351 (2000).

[13] R. Jackiw and S. Weinberg, Phys. Rev. D5, 2473 (1972).

[14] I. Bars and M. Yoshimura, Phys. Rev. D6, 374 (1972).

[15] A. Czarnecki, W. J. Marciano, and A. Vainshtein, hep-ph/0212229 (2002).

[16] T. V. Kukhto, E. A. Kuraev, Z. K. Silagadze, and A. Schiller, Nucl. Phys. B371, 567 (1992).

[17] M. Gourdin and E. de Rafael, Nucl. Phys. B10, 667 (1969).

[18] B. E. Lautrup and E. de Rafael, Phys. Rev. 174, 1835 (1968).

[19] M. Davier, S. Eidelman, A. Hocker, and Z. Zhang, Eur. Phys. J. C27, 497 (2003).

[20] R. Alemany, M. Davier, and A. Hocker, Eur. Phys. J. C2, 123 (1998). 
[21] Y.-S. Tsai, Phys. Rev. D4, 2821 (1971).

[22] R. B. for the ALEPH Collaboration, Z. Physik C76, 15 (1997).

[23] M. Davier, S. Eidelman, A. Hocker, and Z. Zhang, Eur. Phys. J. C31, 503 (2003).

[24] S. Ghozzi and F. Jegerlehner, Z. Phyz. C67, 585 (2003).

[25] M. Davier, hep-ex/0312064 (2003).

[26] M. Davier, hep-ph/0312065 (2003).

[27] B. Krause, Phys. Lett. B390, 392 (1997).

[28] M. Knecht and A. Nyffeler, Phys. Rev. D65, (2002).

[29] T. Kinoshita, B. Nizic, and Y. Okamoto, Phys. Rev. D31, (1986).

[30] J. Bijnens, E. Pallante, and J. Prades, Nucl. Phys. B474, 379 (1996).

[31] M. Hayakawa, T. Kinoshita, and A. I. Sanda, Phys. Rev. Lett. 75, 790 (1995).

[32] M. Hayakawa and T. Kinoshita, Phys. Rev. D57, 465 (1996).

[33] M. Hayakawa and T. Kinoshita, hep-ph/0112102 (2001).

[34] M. Hayakawa and T. Kinoshita, Phys. Rev. D66, (2002).

[35] J. Bijnens, E. Pallante, and J. Prades, Nucl. Phys. B626, 410 (2002).

[36] K. Melnikov and A. Vainshtein, hep-ph/0312226 (2003).

[37] V. B. Berestetskii, O. Krohkin, and A. Khlebnikov, ZhETF (USSR) 30, 788 (1956).

[38] J. S. Schwinger, Ann. of Phys. 2, 407 (1957).

[39] G. Charpak et al., Phys. Rev. Lett. 6, 128 (1961).

[40] J. Bailey et al., Nucl. Phys. B150, 1 (1979).

[41] L. H. Thomas, Phil. Mag. 3, 1 (1927).

[42] J. D. Jackson, Classical Electrodynamics, Second Edition (John Wiley \& Sons, New York, 1975).

[43] F. J. M. Farley and E. Picasso, Adv. Ser. Direct. High Energy Phys. 7, 479 (1990).

[44] K. Hagiwara et al., Physical Review D 66, (2002).

[45] W. Liu et al., Phys. Rev. Letters 82, 711 (1999).

[46] BNL Internal Report, Aug. 8, 1962 (unpublished).

[47] J. M. Kindem, Ph.D. thesis, University of Minnesota, 1998. 
[48] A. Yamamoto et al., Nucl. Inst. A491, 23 (2002).

[49] G. T. Danby et al., Nucl. Inst. A457, 151 (1998).

[50] A. P. Grossmann, Ph.D. thesis, University of Heidelberg, 1998.

[51] g-2 Design Report, 3rd Edition.

[52] R. Holsinger and C. Iselin, the CERN-POISSON Program Package (POISCR) User Guide, Geneva, August 1984 (unpublished).

[53] E. Efstathiadis et al., Nucl. Inst. A496, 8 (2003).

[54] Y. K. Semertzidis et al., Nucl. Inst. A503, 458 (2003).

[55] H. Wiedermann, Particle Acclerator Physics (Springer-Verlag, New York, 1993).

[56] Y. Orlov, C. Özben, and Y. K. Semertzidis, Nucl. Inst. A482, 767 (2003).

[57] W. H. Press et al., Numerical Recipes in C (Cambridge University Press, New York, 1992).

[58] Vector Fields Ltd., OPERA, Electromagnetic Fields analysis Program.

[59] E. Efstathiadis et al., g-2 Note 286 (unpublished).

[60] Y. K. Semertzidis, g-2 Note 149 (unpublished).

[61] C. Kittel, Introduction to Solid State Physics (John Wiley and Sons, Inc., New York, 1996).

[62] R. Prigl et al., Nucl. Inst. A374, 118 (1996).

[63] X. Fei, V. W. Hughes, and R. Prigl, Nucl. Inst. A394, 349 (1997).

[64] J. Ouyang, W. E. Earle, J. P. Miller, and W. A. Worstell, Nucl. Inst. A374, 215 (1996).

[65] S. A. Sedykh et al., Nucl. Inst. A455, 346 (2000).

[66] L. H. Duong, Ph.D. thesis, University of Minnesota, 2001.

[67] W. R. Leo, Techniques for Nuclear and Particle Physics Experiments, Second Revised Edition (Springer-Verlag, New York, 1994).

[68] IEEE 1014 - 1987 standard.

[69] See, for example, http://www.windriver.com/products/vxworks5/index.html.

[70] See, for example, http://www.linux.org/.

[71] R. Ball, Y. Takeuchi, M. Nomachi, and C. Timmermans, UNIDAQ Version 2.3, 1995.

[72] See, for example, http://wwwasdoc.web.cern.ch/wwwasdoc/hbook/HBOOKMAIN.html. 
[73] See, for example, http://cernlib.web.cern.ch/cernlib/.

[74] See, for example, http://root.cern.ch/.

[75] I. Logashenko, g-2 Note 369 (unpublished).

[76] I. Logashenko, g-2 Note 378 (unpublished).

[77] R. M. Carey et al., Phys. Rev. Lett. 82, 1632 (1999).

[78] H. N. Brown et al., Phys. Rev. D 62, 091101 (2000).

[79] H. N. Brown et al., Phys. Rev. Lett. 86, 2227 (2001).

[80] G. W. Bennett et al., Phys. Rev. Lett. 89, 101804 (2002).

[81] W. D. Phillips, W. E. Cooke, and D. Kleppner, Phys. Rev. Lett. 35, 1619 (1975).

[82] W. D. Phillips, W. E. Cooke, and D. Kleppner, Metrologia 13, 179 (1977).

[83] B. W. Petley and R. W. Donaldson, Metrologia 20, 81 (1980).

[84] H. Deng et al., to be published as a g-2 internal note. (unpublished).

[85] H. Deng et al., g-2 Note 432 (unpublished).

[86] J. Paley, g-2 Note 414 (unpublished).

[87] Private discussion with Paul Debevec.

[88] C. Polly, note published on http://www.npl.uiuc.edu/ polly/g-2/ (unpublished).

[89] C. S. Özben and Y. K. Semertzidis, g-2 Note 365 (unpublished).

[90] I. Logashenko, distributed Note, Oct. 28, 2003 (unpublished).

[91] J. Paley, g-2 Note 409 (unpublished).

[92] F. James, MINUIT Reference Manual, Version 94.1.

[93] M. Deile, internal g-2 Document (unpublished).

[94] J. Paley, g-2 Note 443 (unpublished).

[95] Y. Orlov, g-2 Note 335 (unpublished).

[96] Y. K. Semertzidis, g-2 Note 426 (unpublished).

[97] Private discussions with Ivan Logashenko.

[98] I. Logashenko, distributed Note, Jan. 23, 2003 (unpublished).

[99] W. Morse, g-2 Note 429 (unpublished).

[100] Q. Peng, R. Carey, and J. Miller, distributed note, Jan. 2004 (unpublished). 
[101] Private communications with Qinzeng Peng.

[102] M. Deile, g-2 Note 421 (unpublished).

[103] Robert Carey, private communication.

[104] Private discussions with Gerco Onderwater.

[105] F. Farley, W. Morse, and J. Paley, g-2 Note 445 (unpublished).

[106] Private discussions with Robert Carey.

[107] G. W. Bennett et al., Measurement of the Negative Muon Anomalous Magnetic Moment to 0.7 ppm, hep-ex/0401008, To be published in Phys. Rev. Lett.

[108] R. S. McNabb, Ph.D. thesis, University of Minnesota, 2003.

[109] C. L. Bennett et al., Astrophys. J. Suppl. 148, 1 (2003).

[110] J. R. Ellis, T. Falk, K. A. Olive, and Y. Santoso, Nucl. Phys. B652, 259 (2003).

[111] Keith Olive, University of Minnesota (olive@physics.umn.edu).

[112] K. D. Lane, hep-ph/0102131 (2001).

[113] M. Besançon, hep-ph/0106165 (2001).

[114] M. L. Graesser, Phys. Rev. D61, (2000).

[115] A. G. Denig, hep-ex/0311012 (2003).

[116] Internal muon $g-2$ collaboration discussion.

[117] R. Carey et al., Letter of Intent: An Improved Muon ( $g$-2) Experiment at J-PARC (unpublished).

[118] F. Farley, C. Özben, J. Pretz, and Y. K. Semertzidis, g-2 Note 377 (unpublished). 